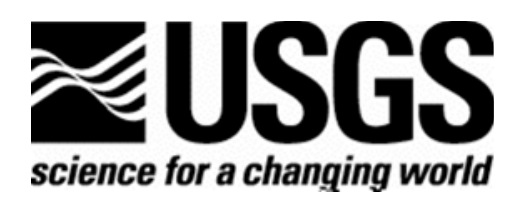

\title{
Schlumberger Soundings in the Long Beach-Wilmington Area, County of Los Angeles, California
}

By Robert J. Bisdorf and David V. Fitterman

Any use of trade, firm, or product names is for descriptive purposes only and does not imply endorsement by the U.S. Government

\section{Open-File Report 2004-1053}




\title{
Schlumberger Soundings in the Long Beach- Wilmington Area, County of Los Angeles, California
}

\author{
By Robert J. Bisdorf and David V. Fitterman
}

\section{Introduction}

In 1999, 2000, and 2001 the U.S. Geological Survey made 78 direct current (dc) electrical soundings in the Long Beach-Wilmington area, California (Figure 1) using the Schlumberger array. The soundings were made to determine the subsurface resistivity distribution, and to correlate areas of low resistivity with intrusive seawater. The purpose of this report is to present the data, its interpretation, cross sections of interpreted resistivity, and maps of interpreted resistivity at selected depths.

Figure 2 is a map of the sounding locations. The soundings are represented by filled circles. The 1999 data are numbered 1 through 21, the 2000 data are numbered 23 through 42, and the 2001 data are numbered 43 through 78 . Table 1 gives the sounding number, $x$ and $y$ coordinates of the soundings in kilometers relative to the Universal Transverse Mercator projection zone 11, 1927 North American Datum.

The data were interpreted using an automatic computerized interpretation program (Zohdy and Bisdorf, 1989) written for IBM PC's and compatible computers. The soundings are designated 1 through 78. For each sounding curve, the data in the Appendix includes:

1. A sounding title designated by the name of the survey area followed by the sounding number.

2. A tabulation of the $A B / 2$ electrode spacings (in meters and feet) and corresponding apparent resistivities (in ohm-meters).

3. A log-log plot of the field data points. Each set of measurements that were made with the same potential electrode spacing (MN) is connected with a solid line. Measurements were made at MN/2 spacings of $0.6,2,20,60$, and $200 \mathrm{ft}$. as appropriate.

4. A tabulation of the automatically interpreted layering, with depths in meters and feet and the corresponding resistivities in ohm-meters. Several sounding titles have an "S" suffix that indicates that the field data were smoothed before the inversion was performed.

5. A log-log plot of the results of the automatic interpretation program. The circles represent the shifteddigitized field data, the continuous curve represents the sounding curve calculated from the interpreted layering, and the step-function curve represents the interpreted layering.

The Long Beach-Wilmington area is highly urban, making the selection of sounding sites difficult. Areas chosen included parks, edges and medians of freeways, railroad right of ways, and along canals, rivers and drainages. In essence, any place where bare ground was found was tried. Care was taken to minimize the effects of man-made influences such as pipelines, power lines, railroad tracks, etc. Even with our best efforts some of the sounding curves were distorted due to these cultural influences. The maps and cross section presented are from soundings that have had these cultural distortions manually removed. The distorted soundings were made to resemble nearby undistorted soundings. 


\section{CROSS SECTIONS OF INTERPRETED RESISTIVITY}

Resistivity cross sections are generated from individual sounding interpretations. Each sounding interpretation is sampled in a manner to approximate a continuous vertical distribution of resistivity with depth (Bisdorf, 1982). These vertical distributions are then horizontally interpolated to create a grid. Colors are assigned based on the interpolated resistivity values and the desired contour levels. Triangles on the upper surface of the cross section designate the sounding locations. Topographic information, input as sounding elevations, is represented by connecting the surface location of the soundings by straight lines. A white line in the lower portion of the cross sections represents the last depth that can be attributed to the sounding data. Information below that depth has been generated by the grid generation process and should not be relied upon.

Eight cross sections are presented in Figures 3-10. The first section (Figure 3) from along the Long Beach Freeway and the Los Angeles River is fairly typical of the results. At the north end of the section very resistive near-surface material is seen in the upper $10 \mathrm{~m}$ becoming more conductive with depth. This resistive zone is thought to be dry sand. This pattern is overprinted at the south end of the section where a very conductive $(<4.5 \mathrm{ohm}-\mathrm{m})$ wedge is seen in the depth range of 5 to $60 \mathrm{~m}$, deepening and becoming thinner towards the north. Seawater intrusion is the most likely cause of this low resistivity wedge. The Terminal Island Freeway section (Figure 4) generally has a similar pattern, however, the low resistivity zone extends farther to the north and has lower resistivities than the cross section of Figure 3. Tidal flow of seawater in the Dominguez Channel is the likely cause of the thick low-resistivity zone seen in Figure 5. The cross section to the west of the Dominguez Channel along the Alameda Corridor (Figure 6) shows a similar low-resistivity zone.

Seawater does not intrude as far north on the Wilmington Corridor cross section (Figure 7) or the Gaffey Street-Vermont Avenue cross section (Figure 9). The cross section along the Harbor Freeway (Figure 8) shows sea-water intrusion to the south. The cause of the low-resistivity zone to the north is not clear, but could be due to an old marsh along the unchannelized drainage to the north of Harbor Park.

The interpreted resistivities have been separated into a set of ranges thought to be indicative of lithologic and/or hydrologic conditions found in the area (see Table 1).

Table 1. List of resistivity ranges and possible lithologic/hydrologic correlations for the generalized interpretation of cross sections and resistivity maps.

\begin{tabular}{|l|l|}
\hline Interpreted Resistivity & Lithologic or Hydrologic Correlation \\
\hline Greater than $100 \mathrm{ohm}-\mathrm{m}$ & Mostly-dry sand \\
\hline 30 to $100 \mathrm{ohm}-\mathrm{m}$ & Damp sand \\
\hline 10 to $30 \mathrm{ohm}-\mathrm{m}$ & Some clay or brackish water \\
\hline 4.5 to $10 \mathrm{ohm}-\mathrm{m}$ & Brackish water and/or clay rich \\
\hline Less than $4.5 \mathrm{ohm}-\mathrm{m}$ & Very salty (sea) water saturated \\
\hline
\end{tabular}

\section{RESISTIVITY MAPS AT SELECTED DEPTHS}

Maps of interpreted resistivity at a particular depth were generated by sampling the sounding interpretations at depths determined by the difference between the surface elevation at that sounding and the desired depth. The sampled resistivities and the corresponding location values were gridded using a minimum-curvature algorithm (Webring, 1981). To prevent possible interpolated resistivities of less than zero, the logarithm of the resistivities was used for gridding. Colors were assigned based on the grid value and the desired logarithmically spaced contour levels. Since these maps were raster (pixel) based, a bicubic interpolation program was used to increase the size of the resultant image. An interpolation program was used to resample the grid, as opposed to simply gridding the data at the desired final interval, because the 
minimum-curvature gridding algorithm generates undesirable results if the data are over sampled. Zohdy (1993) uses similar procedures and provides a discussion of the nuances of resistivity map generation. Maps of interpreted resistivity at depths of 0 (surface), 5, 10, 20 and 30 meters are presented

The surface resistivity map (Figure 11) reflects the fairly uniform covering of low water-content sand throughout the region. At 5 meters depth (Figure 12) the interpreted resistivities start to become more conductive in all but the northeast portion of the study area. The low resistivity zone along the Harbor Freeway is evident. The low resistivity zone to the east that stretches from the harbor area northward beyond the Pacific Coast Highway as seen in the 10-meter depth map (Figure 13). This zone becomes broader and reflects the extent of seawater intrusion in Figure 14 (20-meter depth map). In general, the extent of seawater intrusion parallels the shoreline. However, it extends farther inland in the vicinity of the Dominguez Channel. The same pattern persists to a depth of 30 meters (Figure 15).

\section{Summary}

Schlumberger soundings were successfully made in a highly urbanized environment. Site selection was limited by the availability of bare ground and cultural noise. Soundings that exhibited distortion due to nearby cultural interference (pipes, rails, fences) were smoothed to reflect the general shape of nearby undistorted soundings.

In general, interpreted resistivity increases with depth, though at many locations the electrical basement is more conductive than the overlying intermediate layers. Toward the coast, resistivities drop to less than $10 \mathrm{ohm}-\mathrm{m}$ at depths greater than 20 meters. A low resistivity zone is also seen along the Dominguez Channel. The probable cause of the low-resistivity zones is the presence of brine or seawater in the formations.

\section{Literature Cited}

Bisdorf, R.J., 1982, Schlumberger sounding investigations in the Date Creek Basin, Arizona: U.S. Geological Survey Open-File Report 82-953, 55 p.

Webring, Michael, 1981, MINC: A gridding program based on minimum curvature: U.S. Geological Survey Open-File Report 81-1224, $12 \mathrm{p}$.

Zohdy, A. A. R., 1993, Program Kolor-Map \& Section, Amiga Version: U.S. Geological Survey Open-File Report 93-585, 113

p.

Zohdy, A. A. R., and Bisdorf, R. J., 1989, Programs for the automatic processing and interpretation of Schlumberger sounding curves in QuickBASIC 4.0: U.S. Geological Survey Open-File Report, 89-137 A\&B, 64 p. + diskette. 
Table 2 Sounding number and respective $\mathrm{x}$ and $\mathrm{y}$ coordinates in $\mathrm{km}$ relative to the Universal Transverse Mercator projection zone 11, 1927 North American Datum.

\begin{tabular}{|c|c|c|}
\hline $\begin{array}{l}\text { Sounding } \\
\text { number }\end{array}$ & $\begin{array}{l}X \text { coordinate } \\
(\mathrm{km})\end{array}$ & $\begin{array}{l}\text { Y coordinate } \\
(\mathrm{km})\end{array}$ \\
\hline 1 & 386.188 & 3742.135 \\
\hline 2 & 386.319 & 3741.945 \\
\hline 3 & 386.345 & 3741.696 \\
\hline 4 & 386.404 & 3741.457 \\
\hline 5 & 386.445 & 3741.213 \\
\hline 6 & 386.491 & 3740.981 \\
\hline 7 & 387.409 & 3742.485 \\
\hline 8 & 387.308 & 3742.367 \\
\hline 9 & 387.409 & 3742.485 \\
\hline 10 & 387.146 & 3741.378 \\
\hline 11 & 388.420 & 3742.326 \\
\hline 12 & 388.418 & 3742.031 \\
\hline 13 & 388.421 & 3741.728 \\
\hline 14 & 388.426 & 3741.427 \\
\hline 15 & 388.372 & 3739.796 \\
\hline 16 & 388.548 & 3740.013 \\
\hline 17 & 388.560 & 3740.367 \\
\hline 18 & 388.566 & 3740.670 \\
\hline 19 & 387.052 & 3739.488 \\
\hline 20 & 386.362 & 3741.903 \\
\hline 21 & 386.320 & 3742.128 \\
\hline 22 & 387.788 & 3742.957 \\
\hline 23 & 387.123 & 3742.675 \\
\hline 24 & 387.026 & 3742.416 \\
\hline 25 & 387.016 & 3742.117 \\
\hline 26 & 387.014 & 3741.823 \\
\hline 27 & 386.986 & 3741.520 \\
\hline 28 & 386.950 & 3741.326 \\
\hline 29 & 386.986 & 3740.703 \\
\hline 30 & 386.804 & 3740.123 \\
\hline 31 & 386.714 & 3740.020 \\
\hline 32 & 386.541 & 3739.033 \\
\hline 33 & 386.558 & 3738.394 \\
\hline 34 & 381.261 & 3736.447 \\
\hline 35 & 380.353 & 3736.282 \\
\hline 36 & 380.147 & 3737.438 \\
\hline 37 & 379.932 & 3738.066 \\
\hline 38 & 380.968 & 3738.287 \\
\hline 39 & 380.068 & 3738.828 \\
\hline
\end{tabular}

\begin{tabular}{|c|c|c|}
\hline $\begin{array}{l}\text { Sounding } \\
\text { number }\end{array}$ & $\begin{array}{l}X \text { coordinate } \\
(\mathrm{km})\end{array}$ & $\begin{array}{l}\text { Y coordinate } \\
(\mathrm{km})\end{array}$ \\
\hline 40 & 380.637 & 3739.514 \\
\hline 41 & 380.772 & 3739.960 \\
\hline 42 & 380.731 & 3740.544 \\
\hline 43 & 386.752 & 3739.819 \\
\hline 44 & 386.782 & 3739.953 \\
\hline 45 & 386.831 & 3740.112 \\
\hline 46 & 386.872 & 3740.246 \\
\hline 47 & 386.930 & 3740.383 \\
\hline 48 & 387.012 & 3740.594 \\
\hline 49 & 387.068 & 3740.740 \\
\hline 50 & 387.874 & 3741.178 \\
\hline 51 & 387.824 & 3741.046 \\
\hline 52 & 385.079 & 3739.857 \\
\hline 53 & 383.742 & 3740.236 \\
\hline 54 & 383.593 & 3739.295 \\
\hline 55 & 383.642 & 3739.390 \\
\hline 56 & 384.026 & 3738.916 \\
\hline 57 & 384.043 & 3738.988 \\
\hline 58 & 384.031 & 3738.792 \\
\hline 59 & 384.034 & 3738.548 \\
\hline 60 & 384.052 & 3738.237 \\
\hline 61 & 384.152 & 3737.627 \\
\hline 62 & 383.893 & 3737.878 \\
\hline 63 & 381.447 & 3737.008 \\
\hline 64 & 381.523 & 3737.828 \\
\hline 65 & 381.763 & 3737.793 \\
\hline 66 & 381.597 & 3737.976 \\
\hline 67 & 381.566 & 3738.299 \\
\hline 68 & 381.562 & 3738.470 \\
\hline 69 & 381.518 & 3738.803 \\
\hline 70 & 381.460 & 3739.327 \\
\hline 71 & 381.320 & 3739.955 \\
\hline 72 & 393.775 & 3741.154 \\
\hline 73 & 393.856 & 3741.133 \\
\hline 74 & 385.809 & 3741.476 \\
\hline 75 & 385.230 & 3739.661 \\
\hline 76 & 385.083 & 3739.215 \\
\hline 77 & 384.967 & 3738.871 \\
\hline 78 & 384.880 & 3738.590 \\
\hline
\end{tabular}




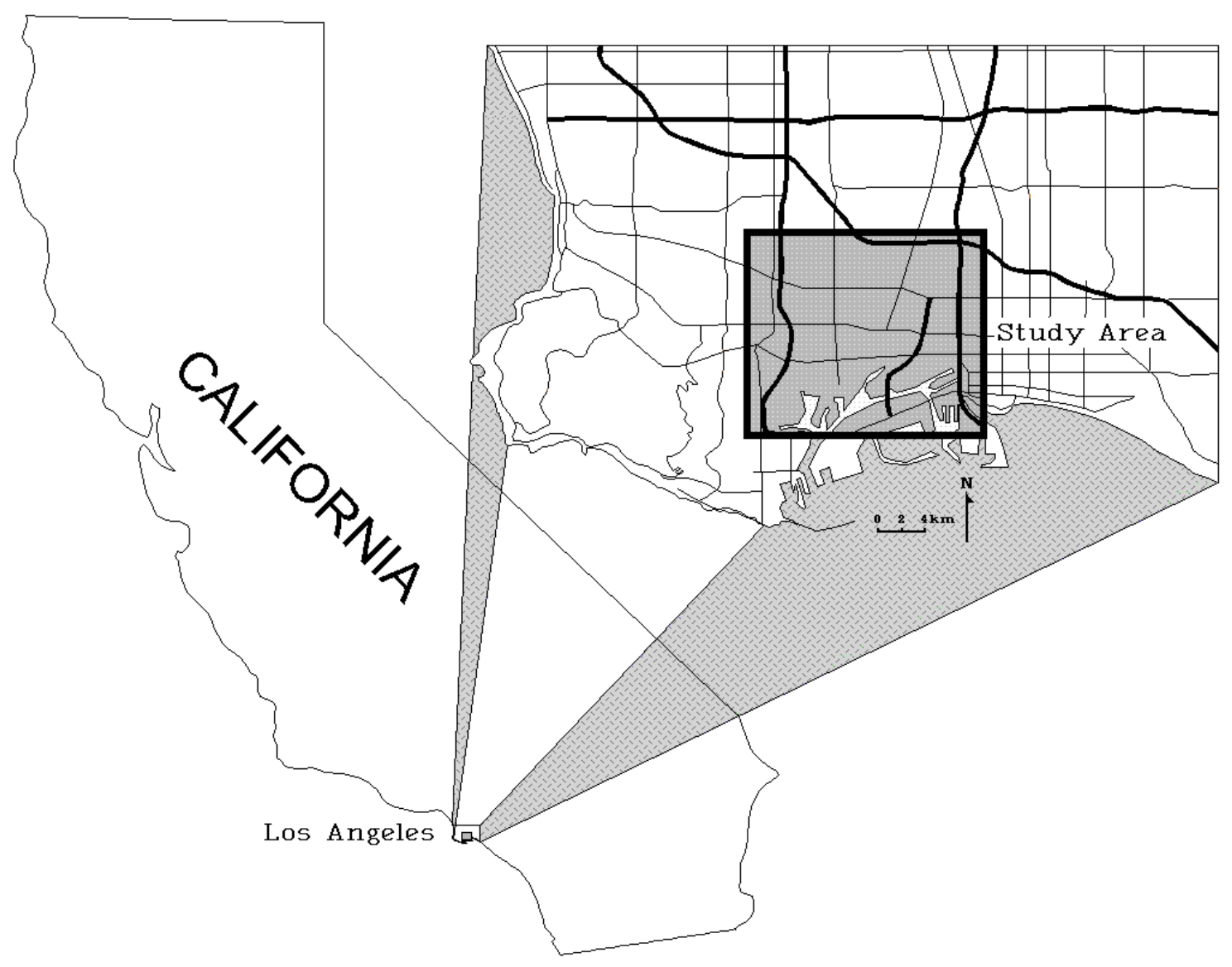

Figure 1 California map showing the location of the study area. 


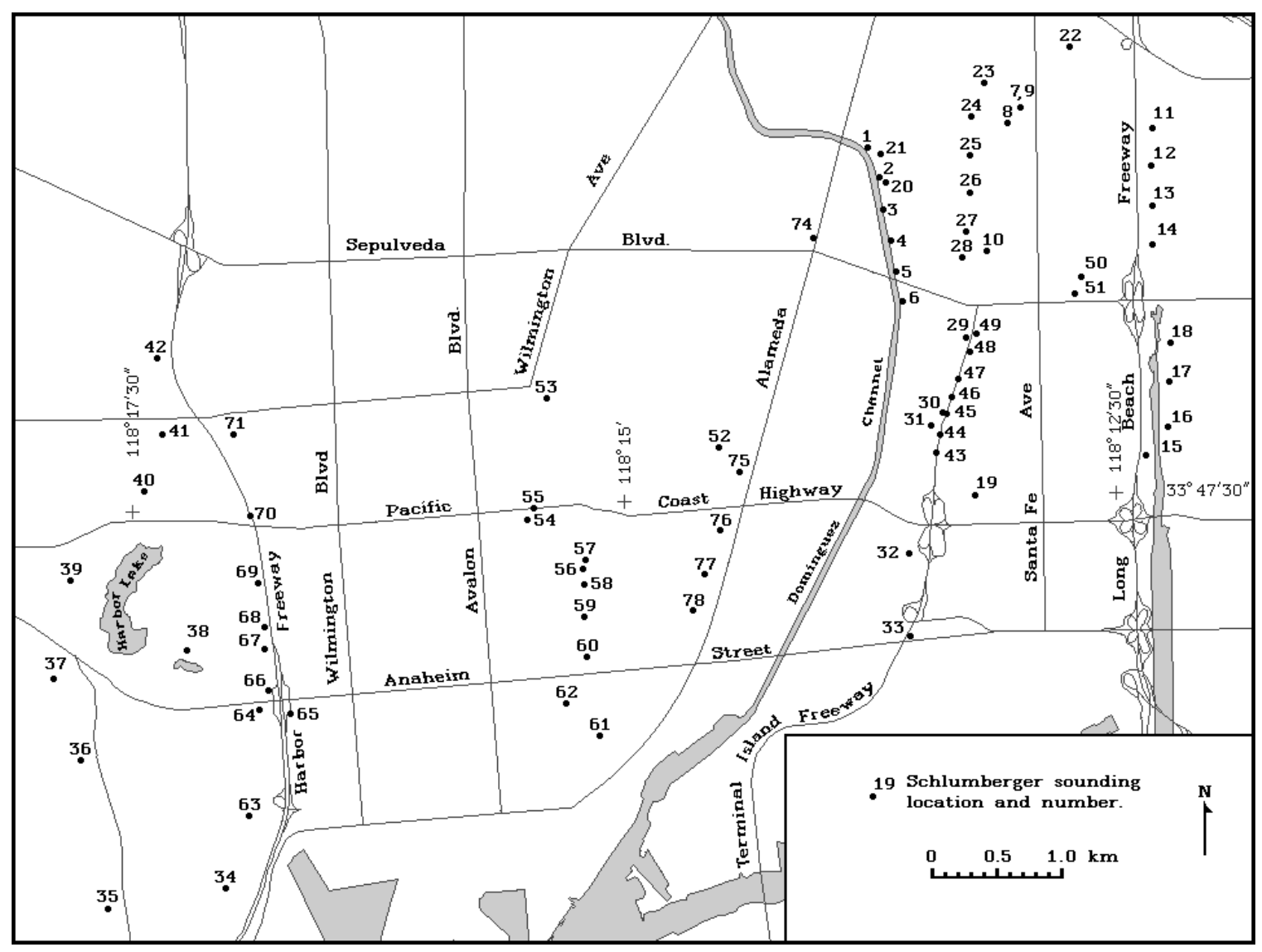

Figure 2 Map showing the location and number of the Schlumberger soundings. 


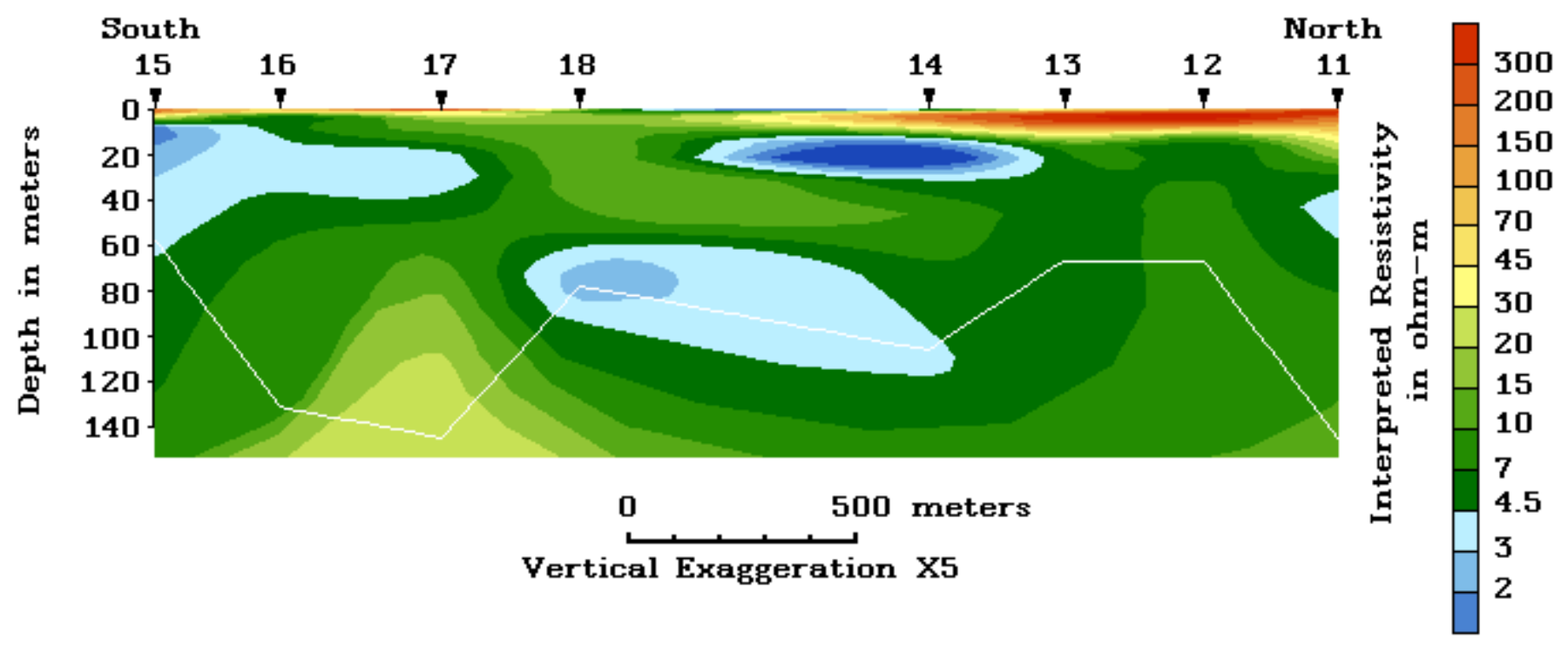

Figure 3 Long Beach Freeway (Los Angeles River) interpreted resistivity cross section. Triangles represent Schlumberger sounding locations. Colors represent interpreted resistivity values. The white line in the lower portion of the cross section represents the last depth that can be attributed to the sounding data. 

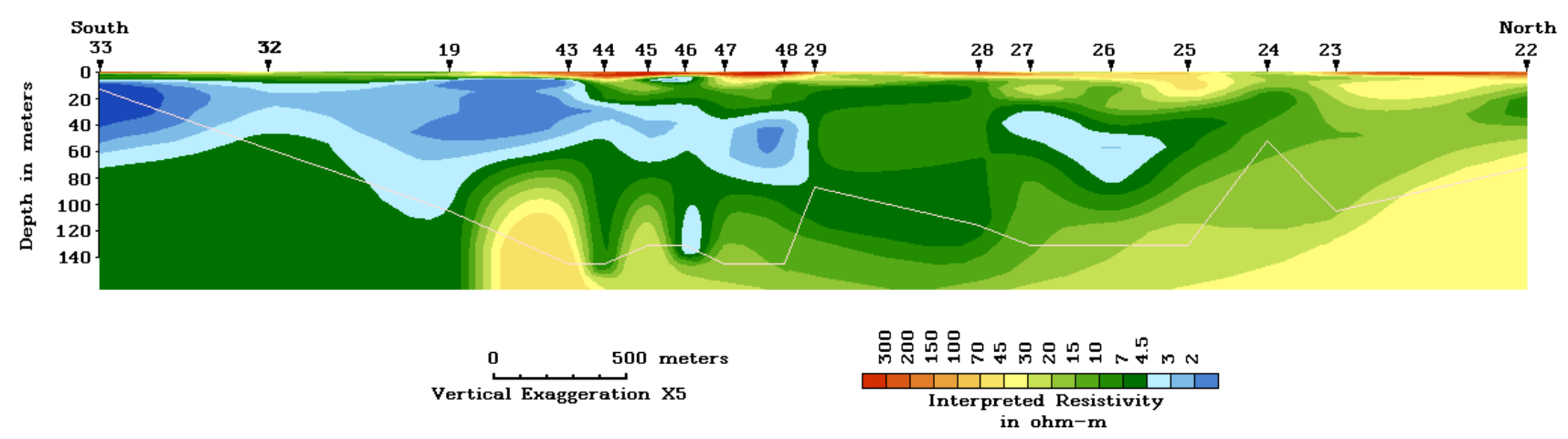

Figure 4 Terminal Island Freeway interpreted resistivity cross section extending northward through the Union Pacific Intermodal Container Transfer Facility (ICTF). Triangles represent Schlumberger sounding locations. Colors represent interpreted resistivity values. The white line in the lower portion of the cross section represents the last depth that can be attributed to the sounding data. 


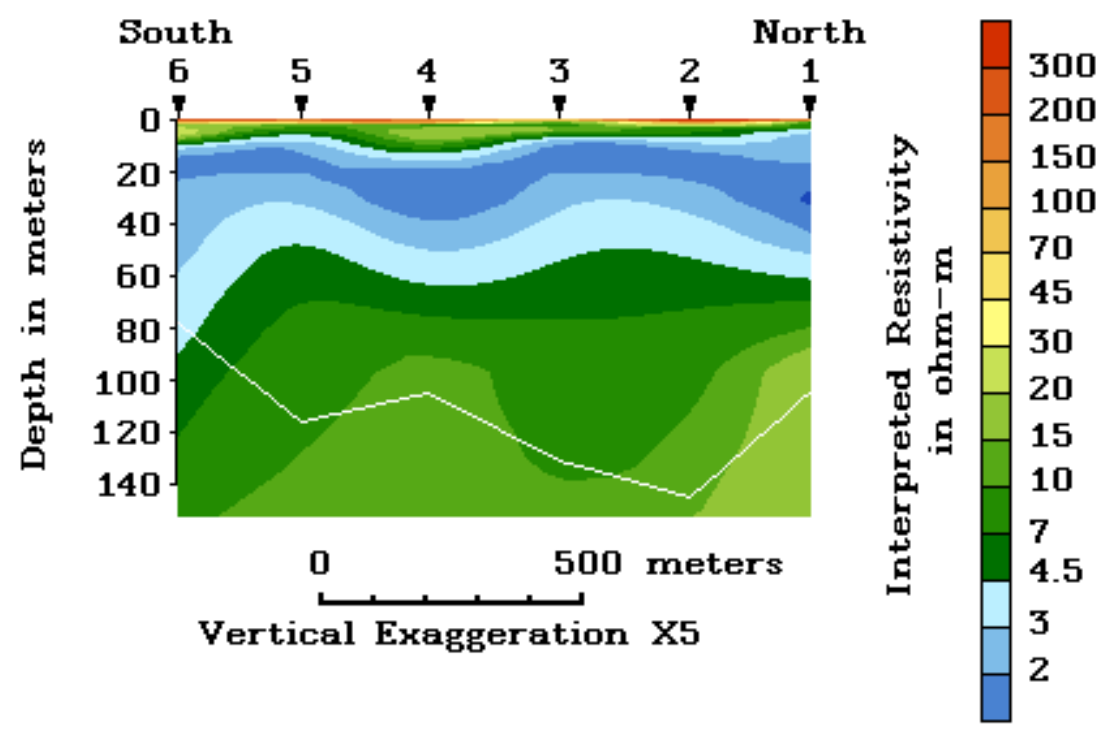

Figure 5 Dominguez Channel interpreted resistivity cross section. Triangles represent Schlumberger sounding locations. Colors represent interpreted resistivity values. The white line in the lower portion of the cross section represents the last depth that can be attributed to the sounding data. 


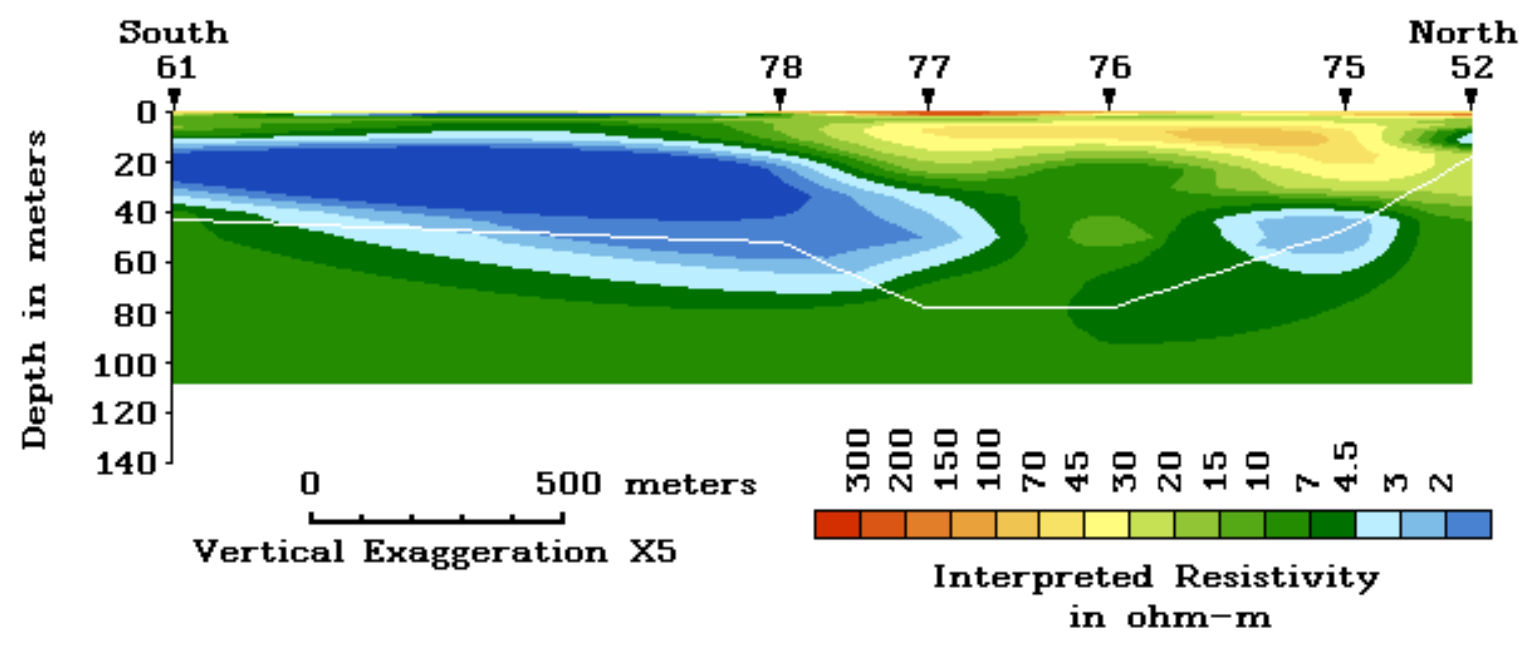

Figure 6 Alameda Corridor interpreted resistivity cross section. Triangles represent Schlumberger sounding locations. Colors represent interpreted resistivity values. The white line in the lower portion of the cross section represents the last depth that can be attributed to the sounding data. 


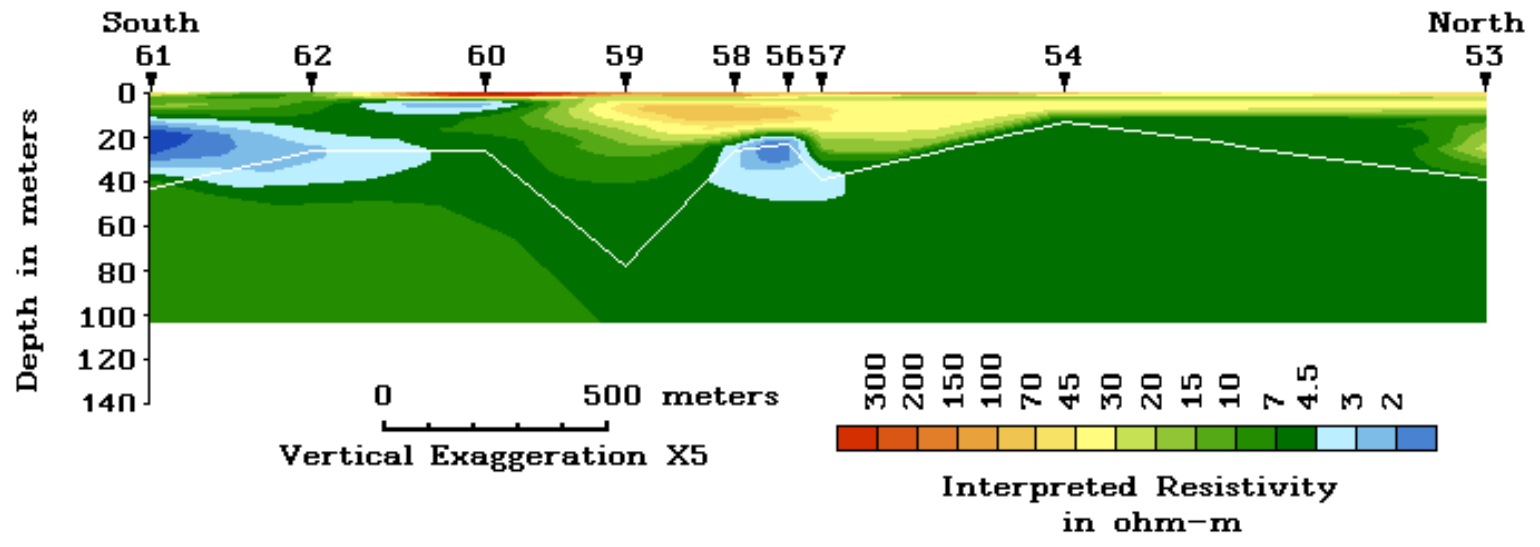

Figure 7 Wilmington Avenue-Avalon Blvd. interpreted resistivity cross section. Triangles represent Schlumberger sounding locations.

Colors represent interpreted resistivity values. The white line in the lower portion of the cross section represents the last depth that can be attributed to the sounding data. 


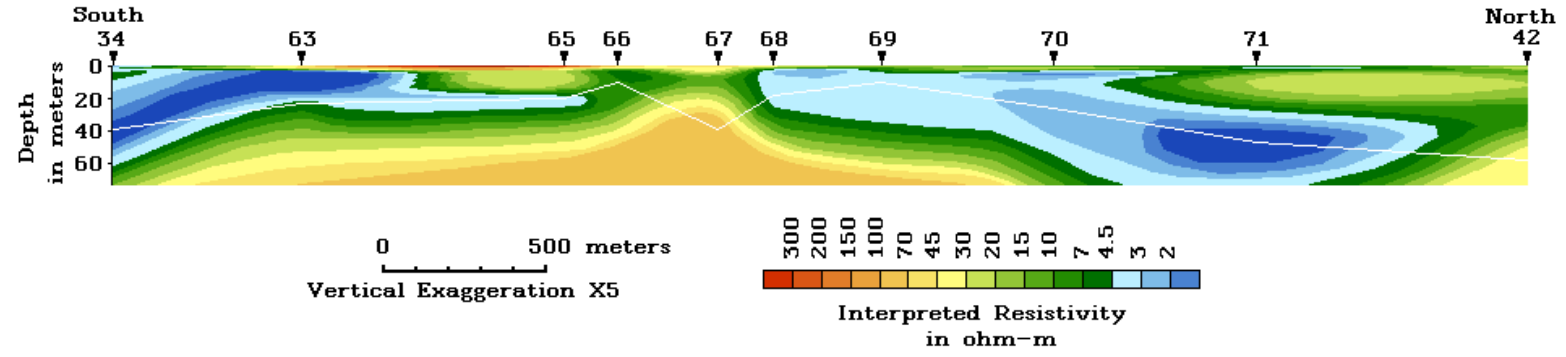

Figure 8 Harbor Freeway interpreted resistivity cross section. Triangles represent Schlumberger sounding locations. Colors represent interpreted resistivity values. The white line in the lower portion of the cross section represents the last depth that can be attributed to the sounding data. 


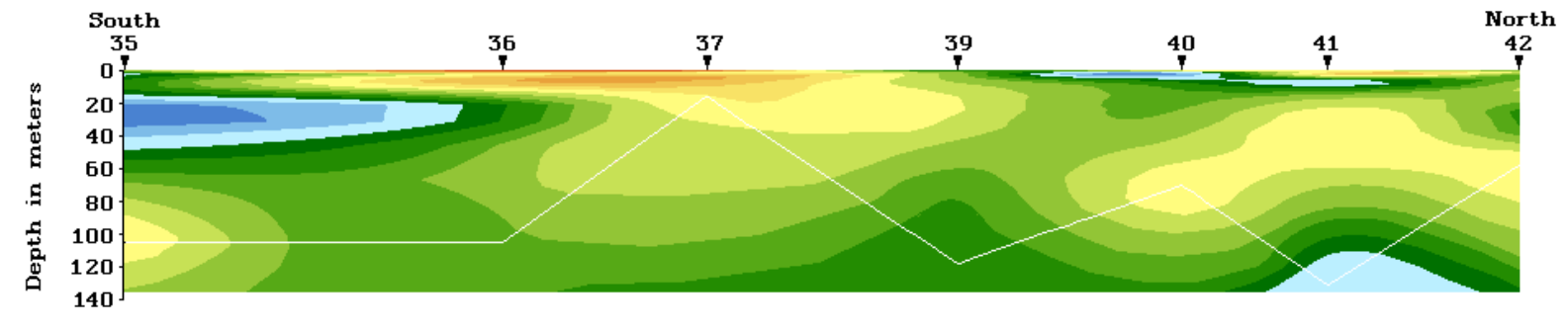

$\mathbf{0}$

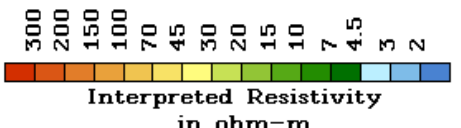

Vertical Exaggeration X5

in ohm-m

Figure 9 Gaffey Street-Vermont Avenue interpreted resistivity cross section. Triangles represent Schlumberger sounding locations. Colors represent interpreted resistivity values. The white line in the lower portion of the cross section represents the last depth that can be attributed to the sounding data. 


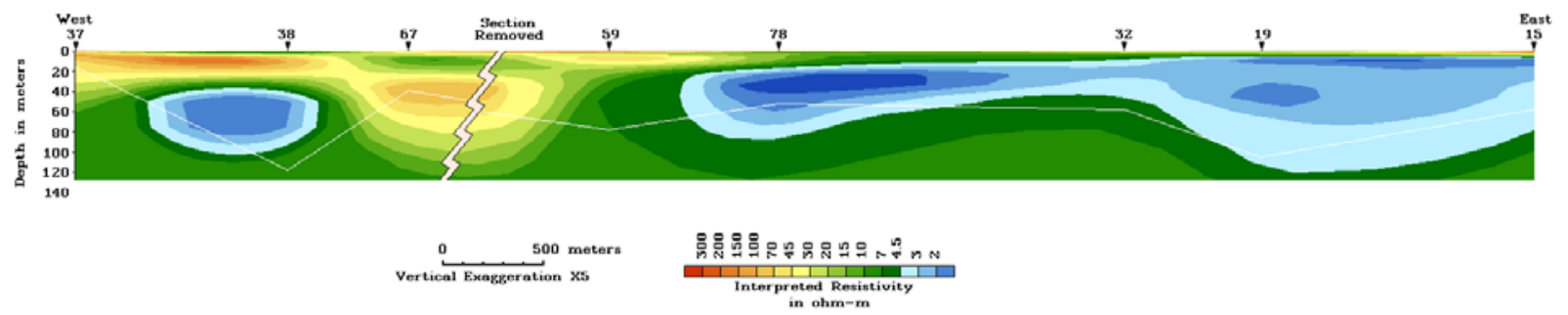

Figure 10 East-west interpreted resistivity cross section through the study area. Triangles represent Schlumberger sounding locations. Colors represent interpreted resistivity values. The white line in the lower portion of the cross section represents the last depth that can be attributed to the sounding data. 


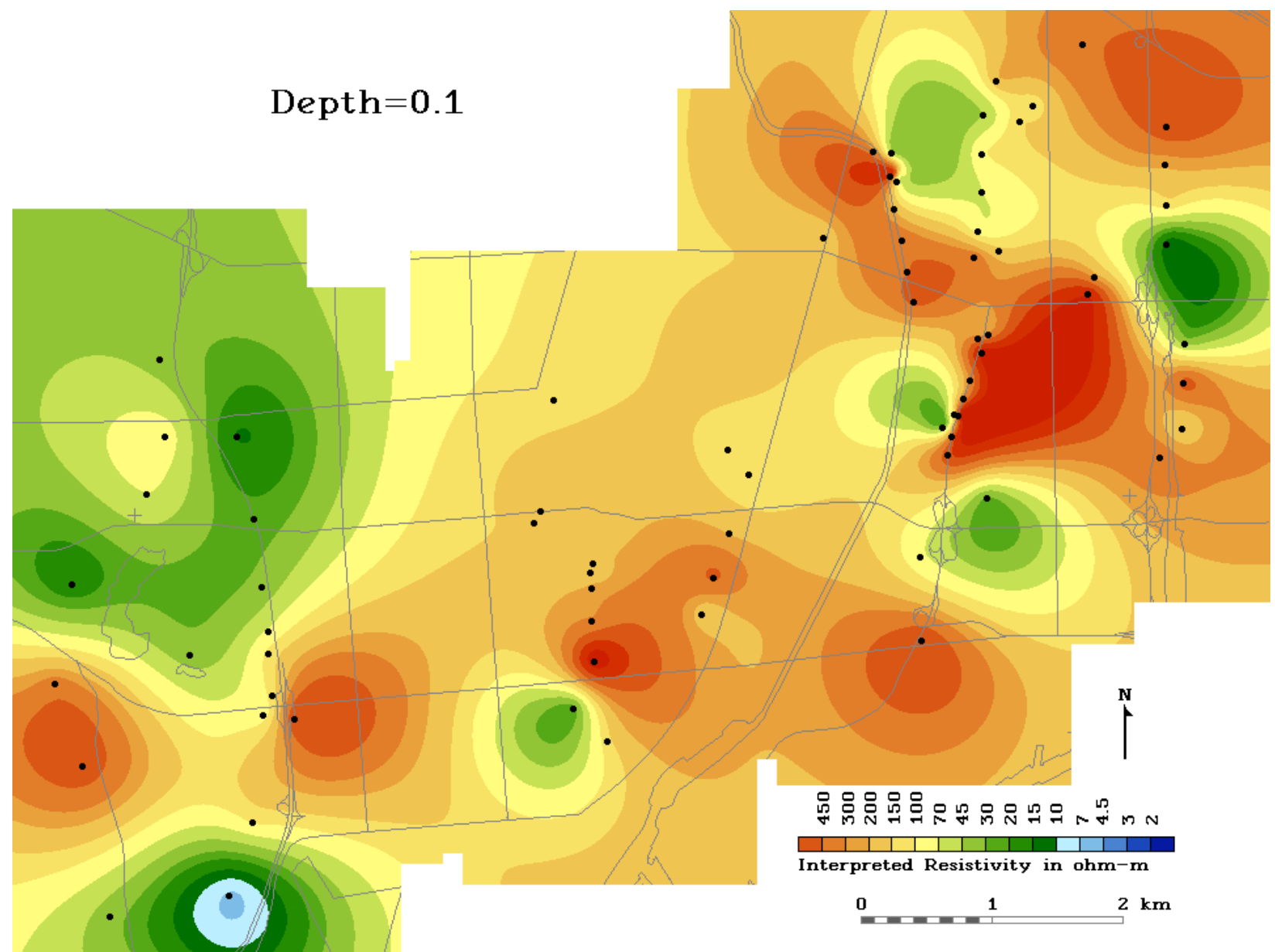

Figure 11 Map of interpreted resistivity at the surface $(0.1 \mathrm{~m})$. Circles represent Schlumberger sounding locations. Colors represent interpreted resistivity values. 


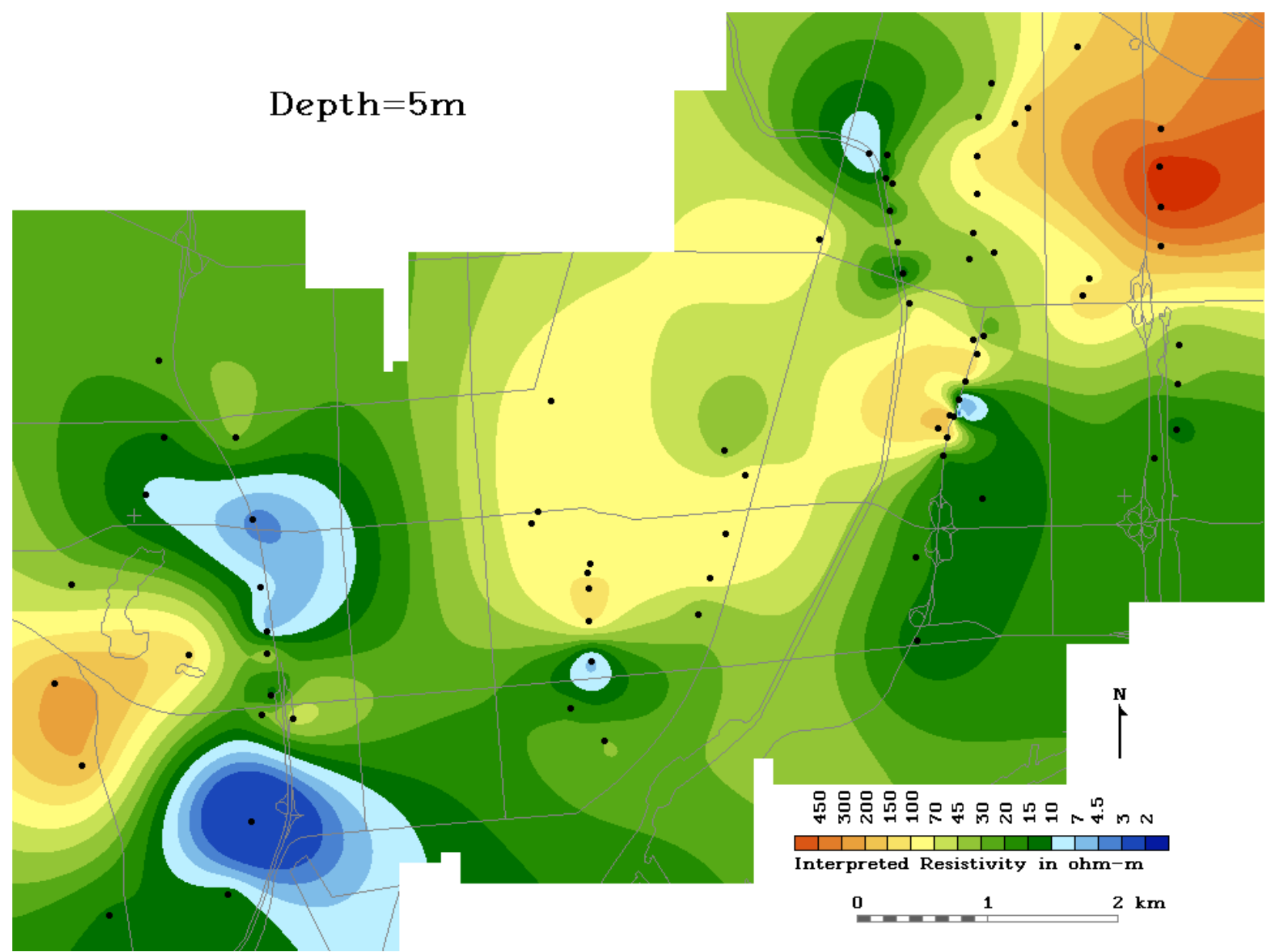

Figure 12 Map of interpreted resistivity at a depth of 5 meters. Circles represent Schlumberger sounding locations. Colors represent interpreted resistivity values. 


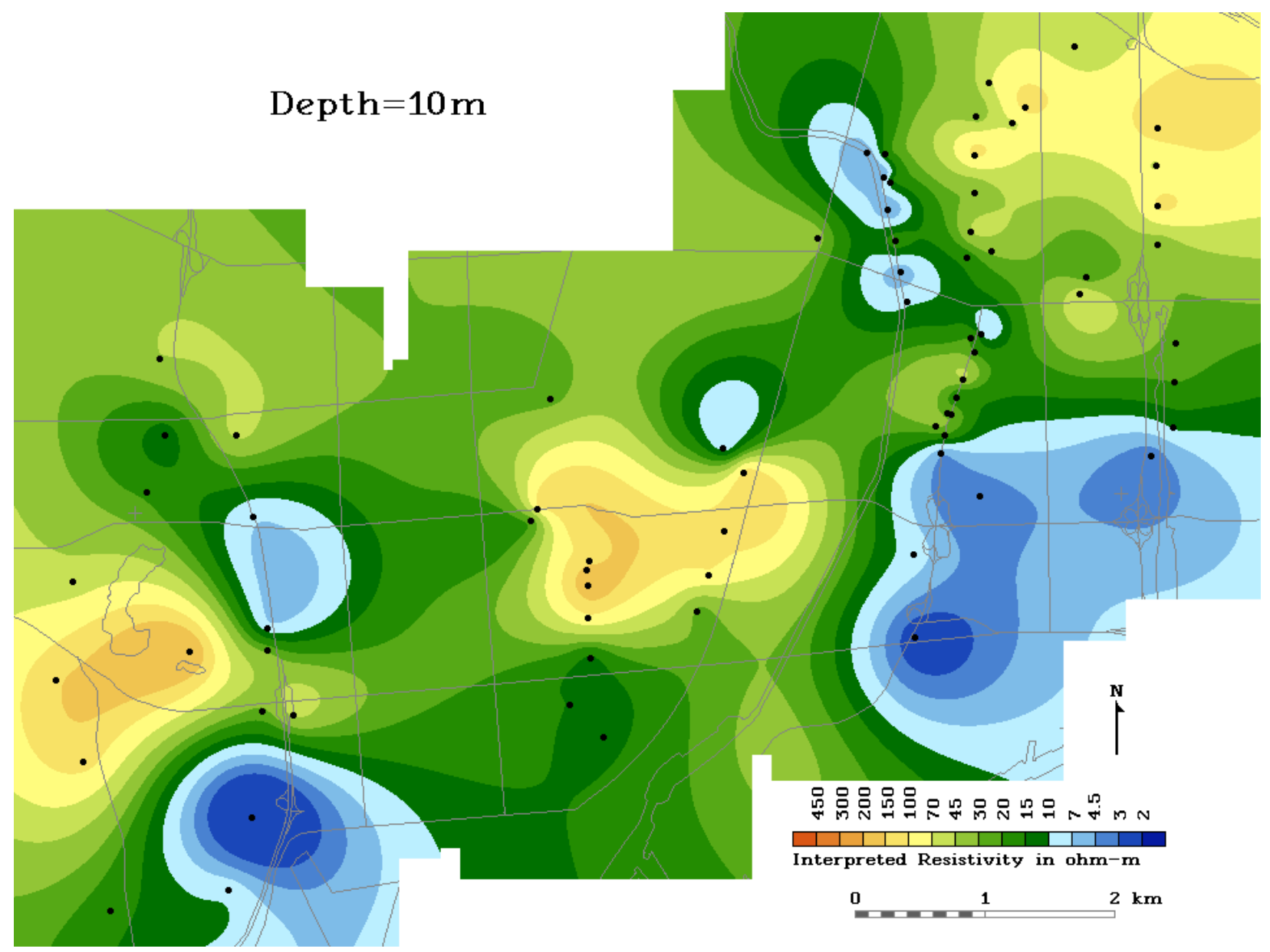

Figure 13 Map of interpreted resistivity at a depth of 10 meters. Circles represent Schlumberger sounding locations. Colors represent interpreted resistivity values. 


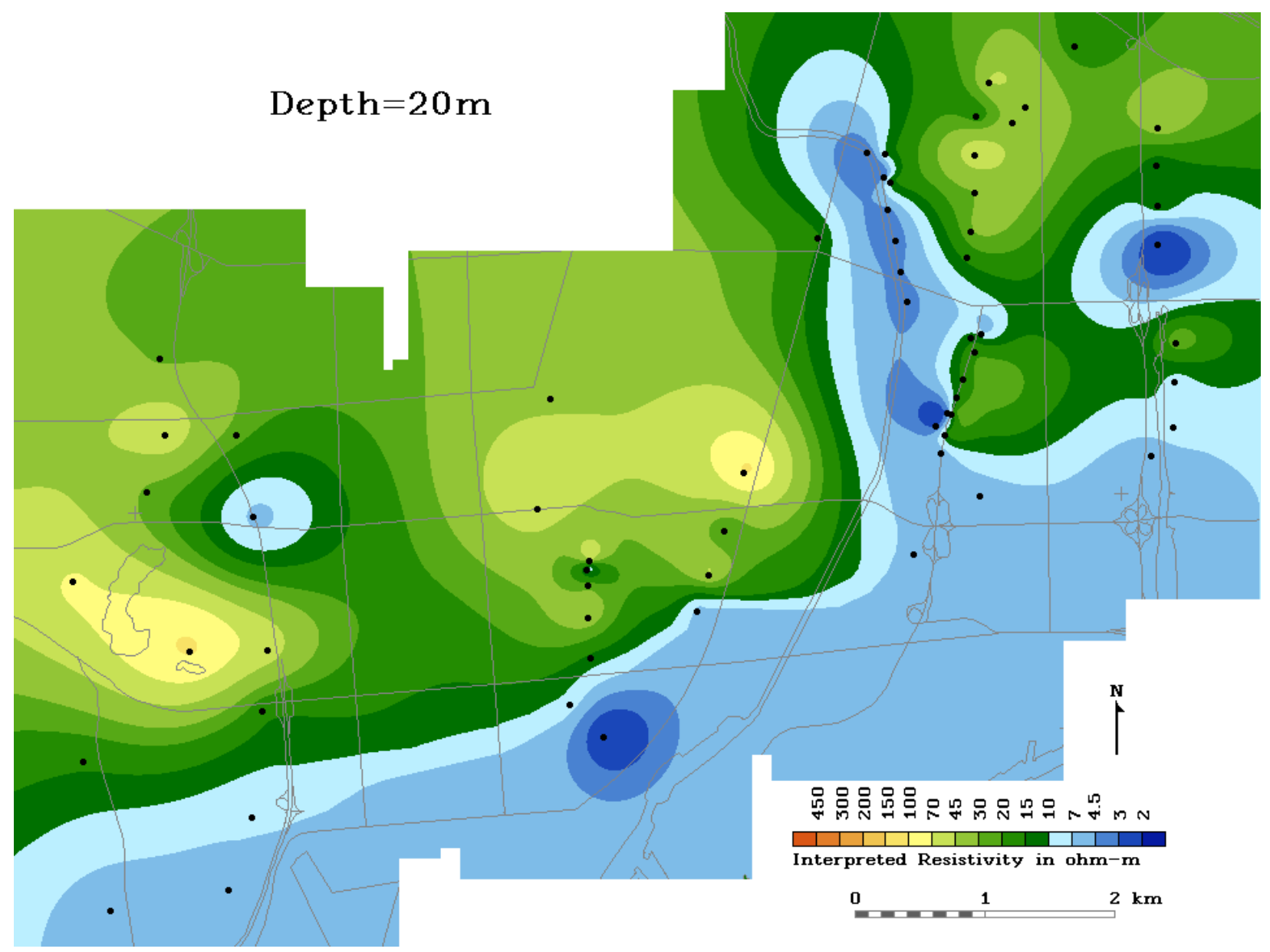

Figure 14 Map of interpreted resistivity at a depth of 20 meters. Circles represent Schlumberger sounding locations. Colors represent interpreted resistivity values. 


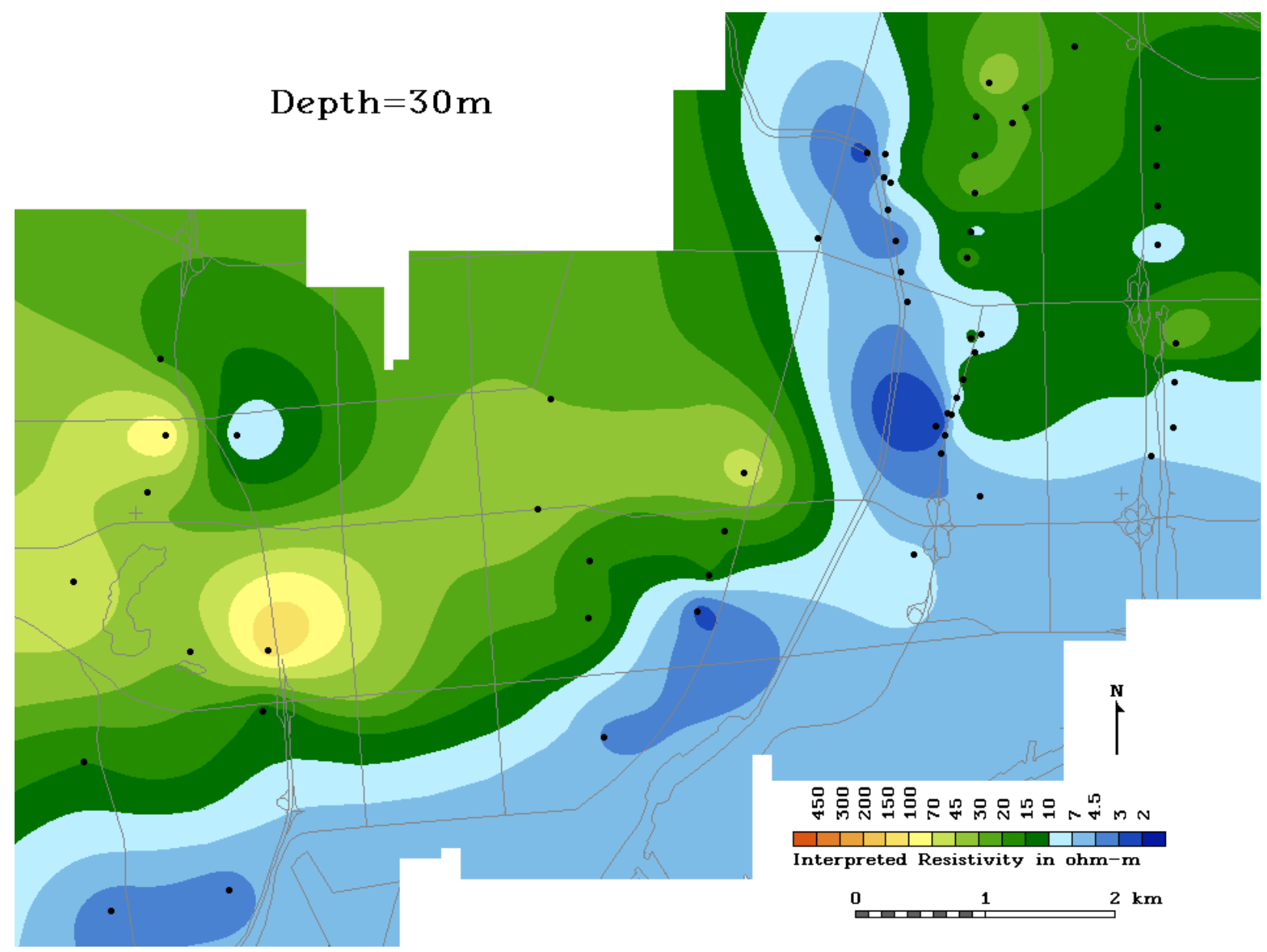

Figure 15 Map of interpreted resistivity at a depth of 30 meters. Circles represent Schlumberger sounding locations. Colors represent interpreted resistivity values. 


\section{APPENDIX}



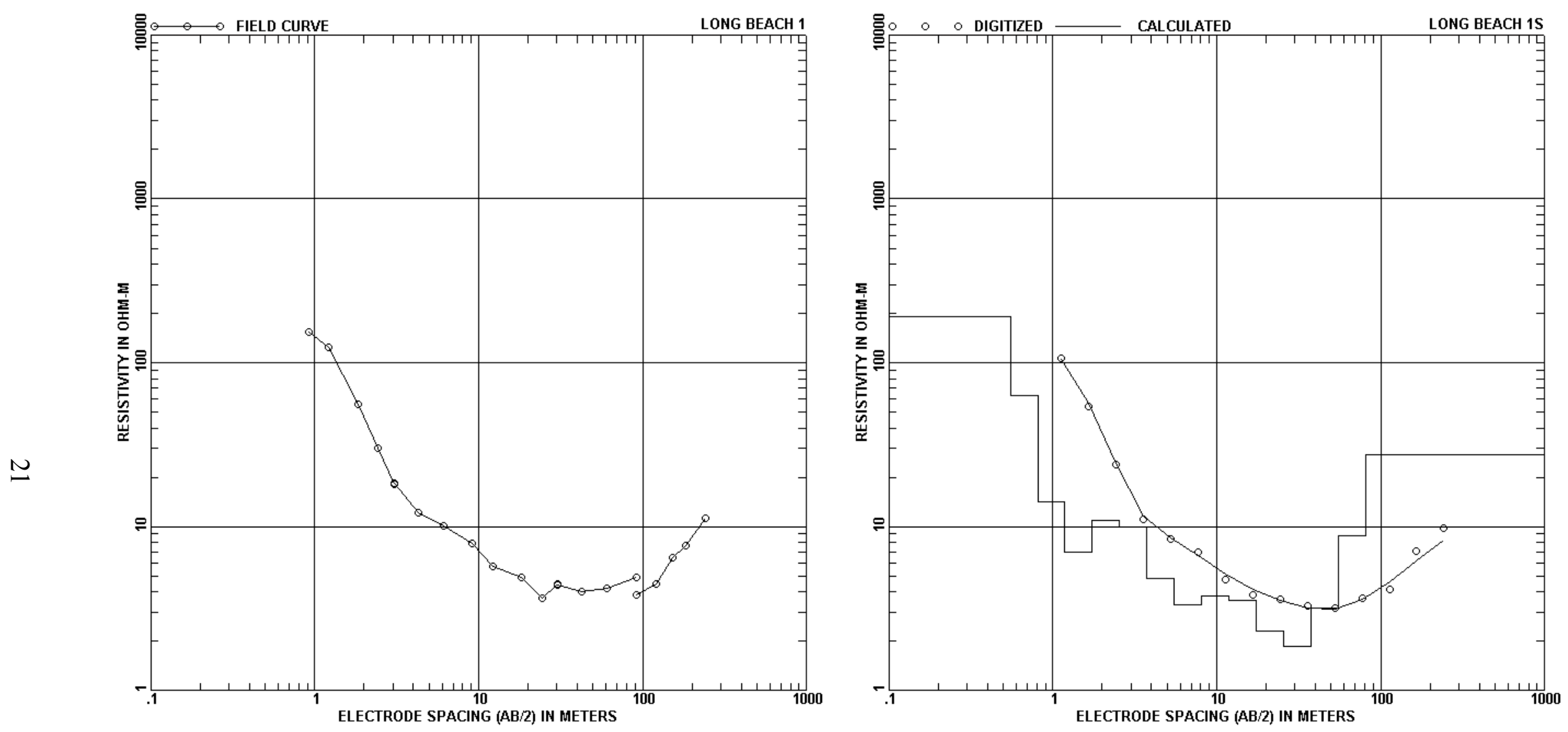

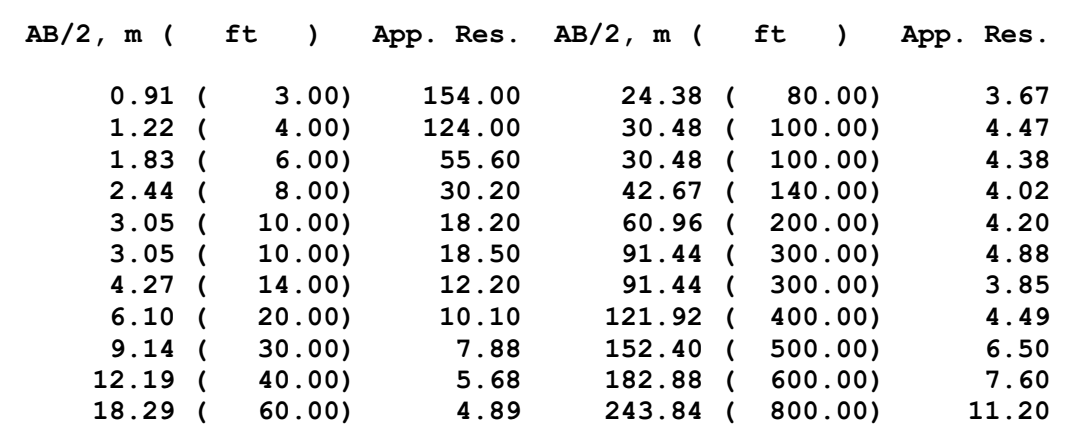

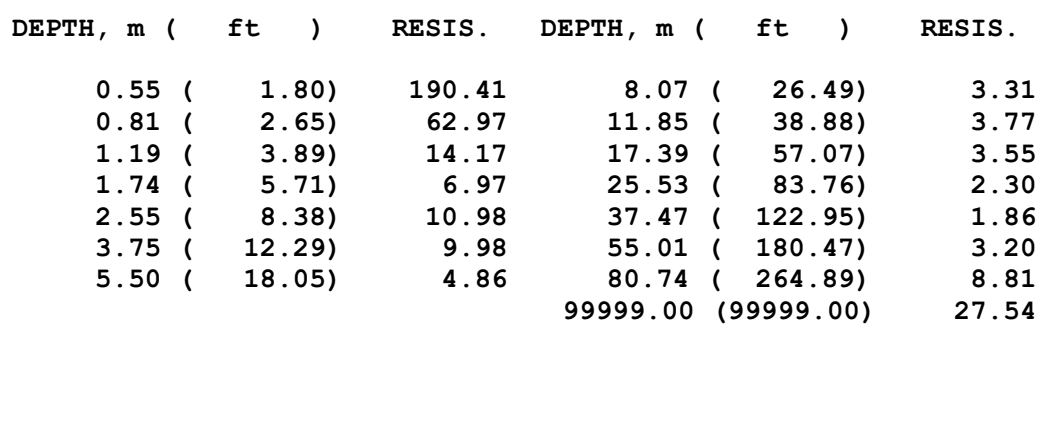



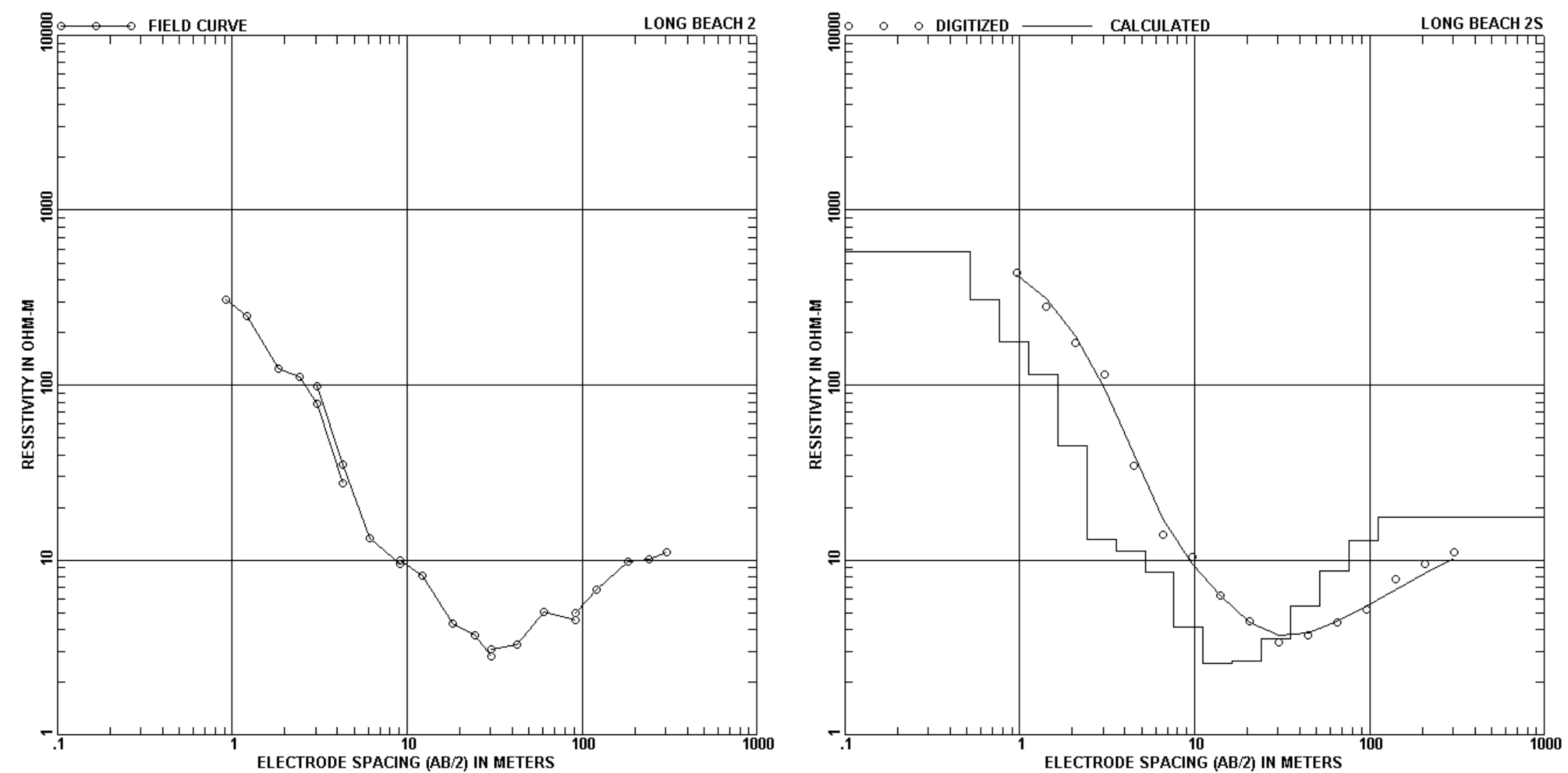

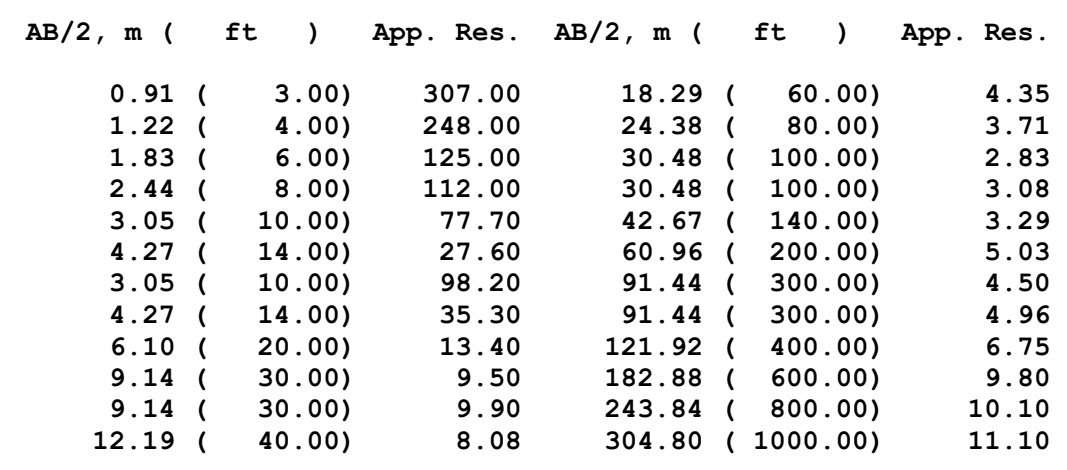

DEPTH, $m$ ( ft ) RESIS, DEPTH, $m($ ft

\begin{tabular}{|c|c|c|c|c|c|}
\hline 0.52 & 1.71) & 576.33 & 11.21 & $36.79)$ & 4.12 \\
\hline 0.76 & $2.51)$ & 308.08 & 16.46 & $54.00)$ & 2.56 \\
\hline 1.12 & $3.68)$ & 177.34 & 24.16 & $79.26)$ & 2.66 \\
\hline 1.65 & $5.40)$ & 114.45 & 35.46 & $116.34)$ & 3.56 \\
\hline 2.42 & $7.93)$ & 44.65 & 52.05 & $170.76)$ & 5.45 \\
\hline 3.55 & $11.63)$ & 13.11 & 76.40 & $250.65)$ & 8.64 \\
\hline 5.20 & $17.08)$ & 11.23 & 112.14 & $367.90)$ & 12.84 \\
\hline 7.64 & $25.06)$ & 8.52 & 99999.00 & $(99999.00)$ & 17.53 \\
\hline
\end{tabular}



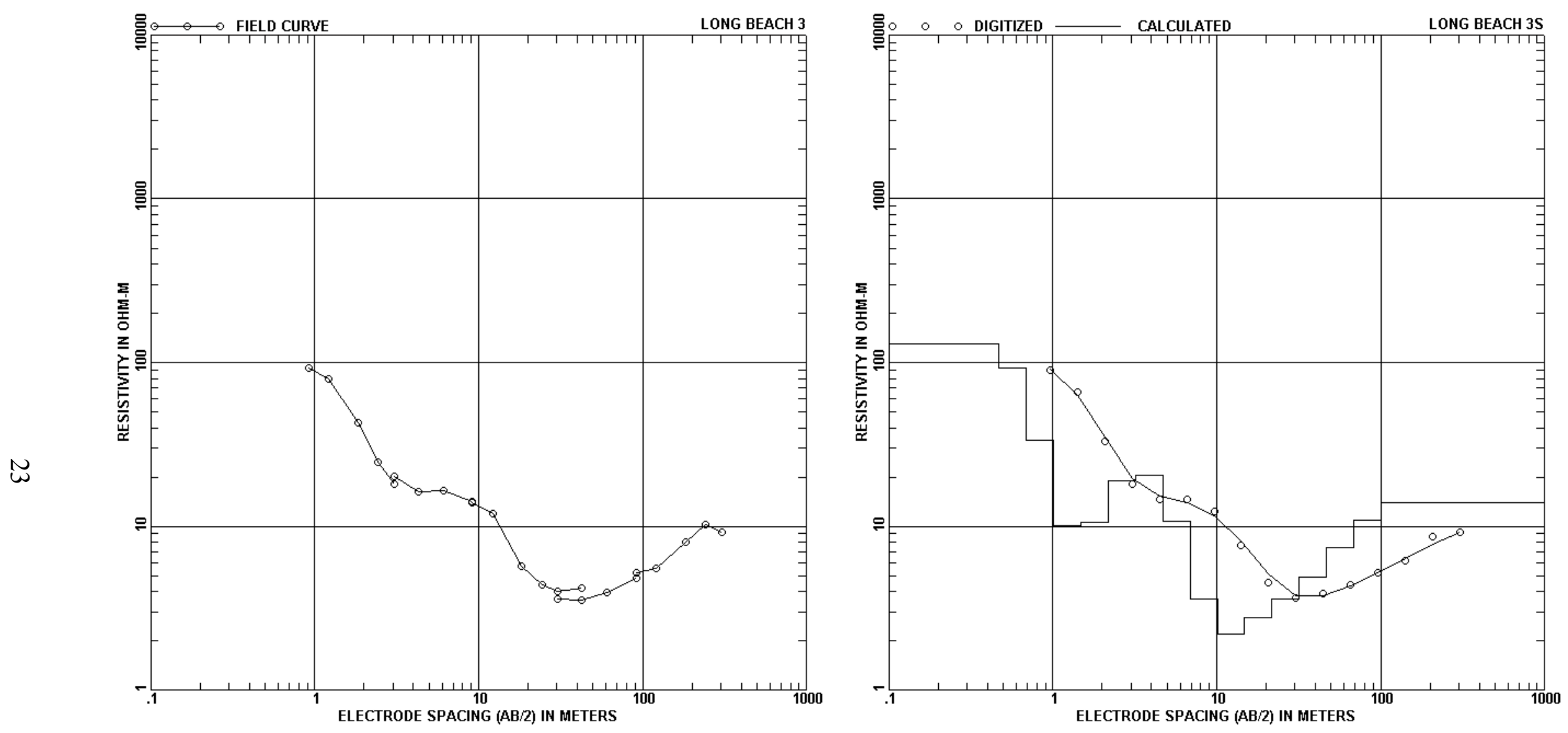

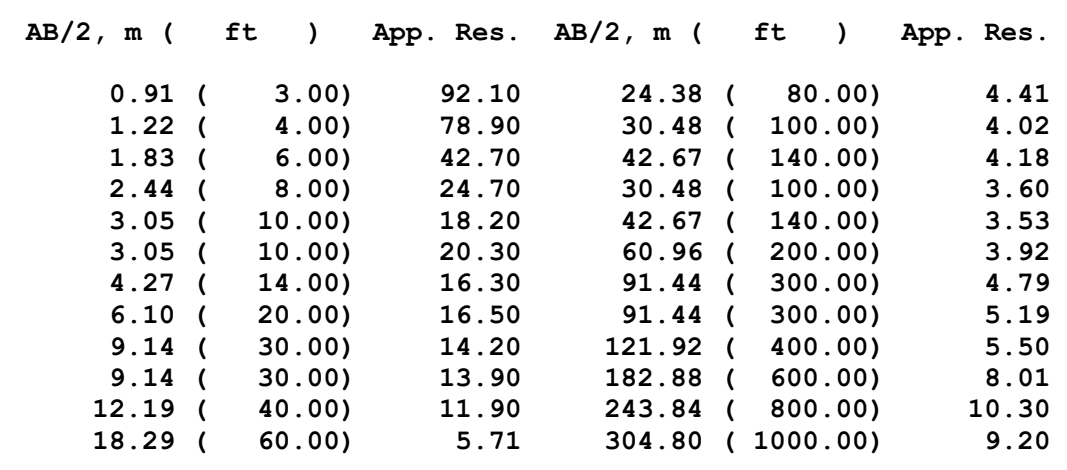

DEPTH, $m$ ( ft ) RESIS, DEPTH, m ( ft

\begin{tabular}{|c|c|c|c|c|c|}
\hline 0.47 & $1.54)$ & 130.42 & 10.09 & $33.11)$ & 3.62 \\
\hline 0.69 & $2.26)$ & 92.57 & 14.81 & $48.60)$ & 2.19 \\
\hline 1.01 & $3.31)$ & 33.67 & 21.74 & $71.34)$ & 2.75 \\
\hline 1.48 & $4.86)$ & 10.07 & 31.91 & $104.71)$ & 3.58 \\
\hline 2.17 & $7.13)$ & 10.56 & 46.84 & $153.69)$ & 4.87 \\
\hline 3.19 & $10.47)$ & 18.90 & 68.76 & $225.58)$ & 7.37 \\
\hline 4.68 & 15.37) & 20.40 & 100.92 & $331.11)$ & 10.87 \\
\hline 6.88 & $22.56)$ & 10.77 & 99999.00 & $(99999.00)$ & 13.95 \\
\hline
\end{tabular}



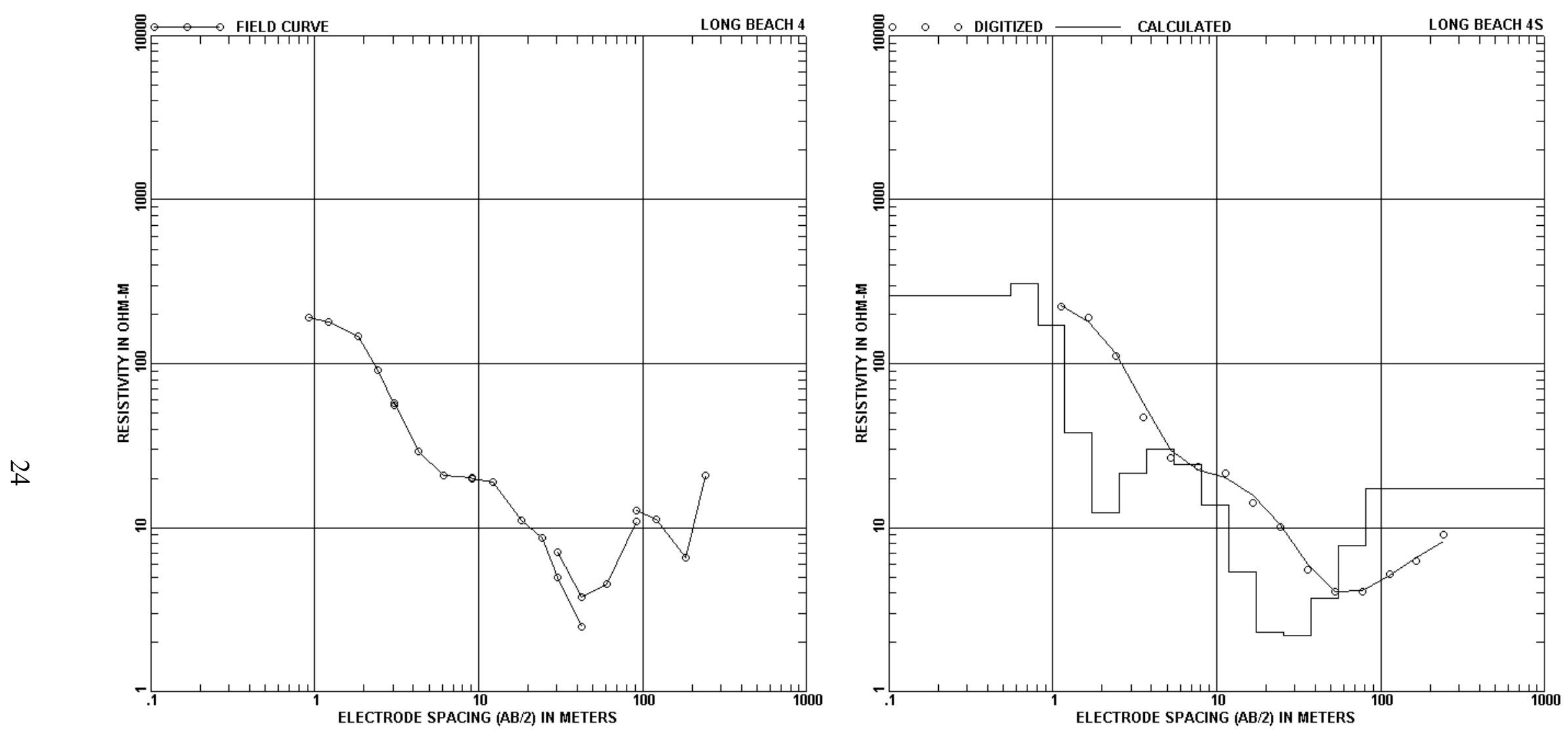

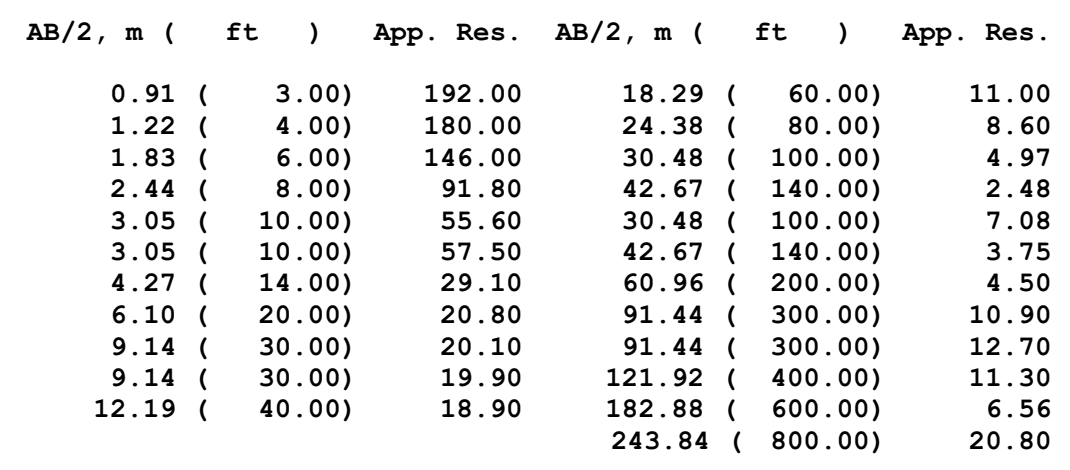

\begin{tabular}{|c|c|c|c|c|c|}
\hline DEPTH， m ( & ft & RESIS. & DEPTH， m ( & ft & RESIS. \\
\hline 0.55 & $1.80)$ & 258.16 & 8.07 & $26.49)$ & 24.26 \\
\hline 0.81 & $2.65)$ & 307.09 & 11.85 & $38.88)$ & 13.72 \\
\hline 1.19 & $3.89)$ & 171.04 & 17.39 & $57.07)$ & 5.39 \\
\hline 1.74 & $5.71)$ & 37.97 & 25.53 & $83.76)$ & 2.29 \\
\hline 2.55 & $8.38)$ & 12.33 & 37.47 & $122.95)$ & 2.21 \\
\hline 3.75 & $12.29)$ & 21.35 & 55.01 & $(180.47)$ & 3.74 \\
\hline 5.50 & $18.05)$ & 30.06 & 80.74 & (264.89) & 7.72 \\
\hline & & & 99999.00 & $(99999.00)$ & 17.24 \\
\hline
\end{tabular}



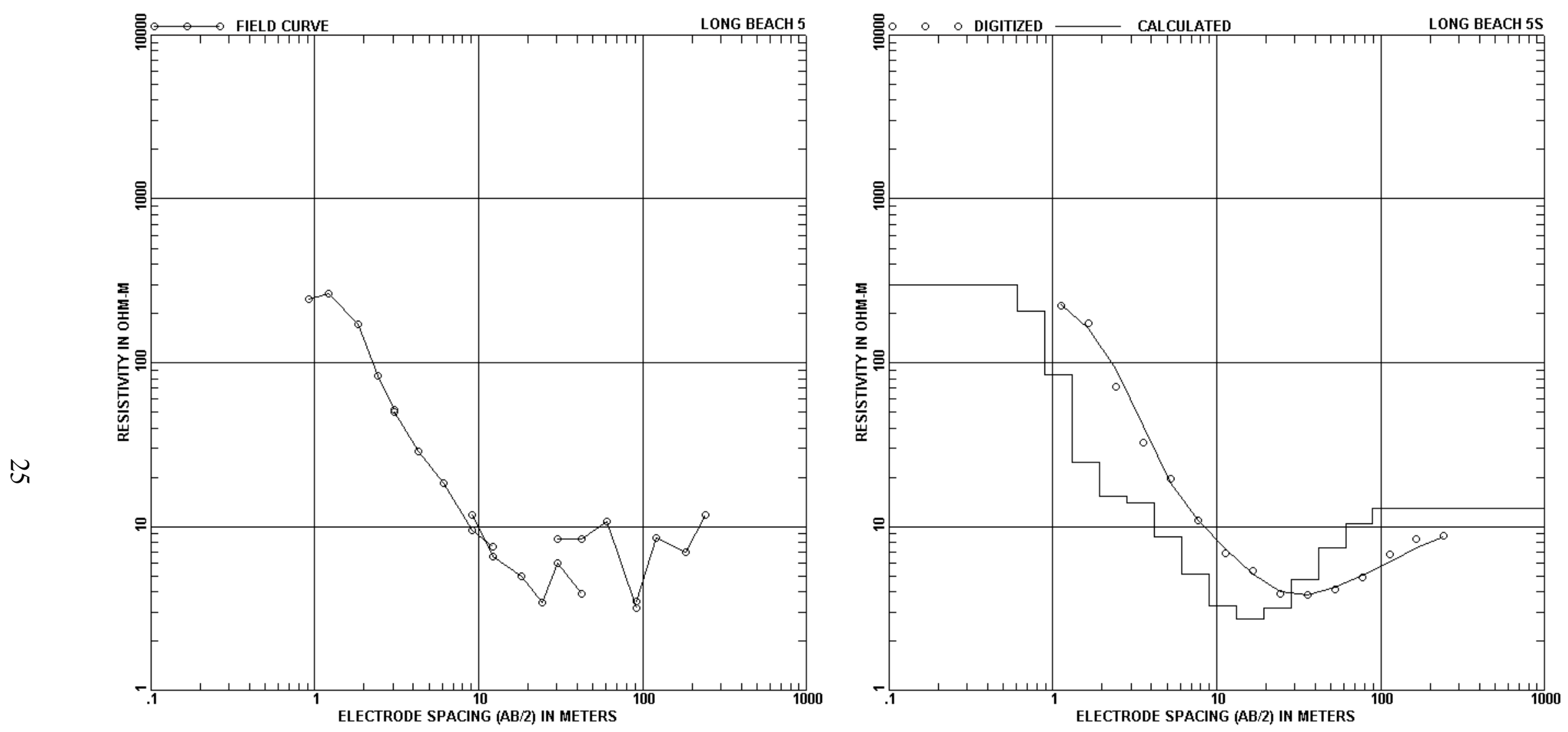

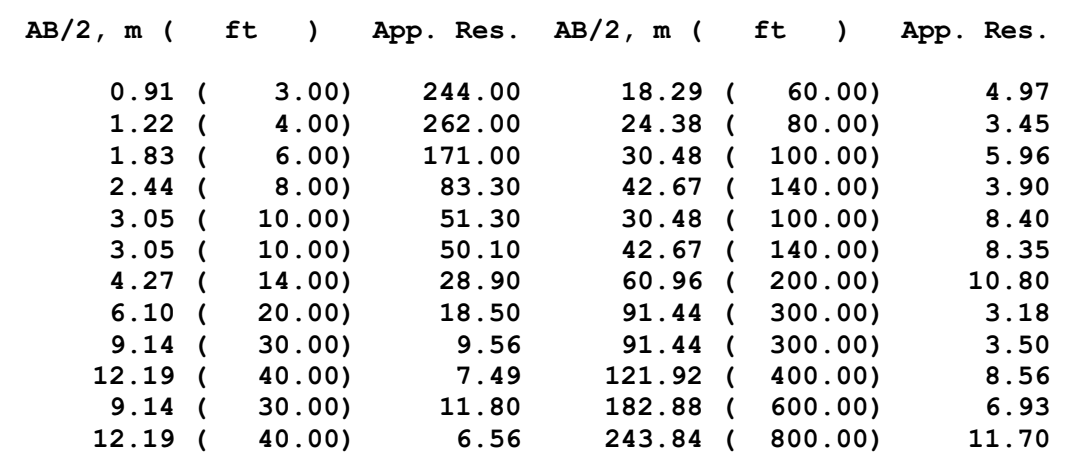

DEPTH, $m$ ( ft ) RESIS. DEPTH, $m$ ( ft

$\begin{array}{rlr}0.61 & ( & 2.01) \\ 0.90 & ( & 2.94) \\ 1.32 & ( & 4.32) \\ 1.93 & ( & 6.34) \\ 2.84 & ( & 9.31) \\ 4.16 & ( & 13.66) \\ 6.11 & ( & 20.05)\end{array}$
297.38
207.81
83.90
24.76
15.22
13.95
8.59

$8.97(29.43)$

$13.17(43.20)$

$19.33(63.41)$

41.64 ( 136.61$)$

$61.64(200.52)$

89.71 ( 294.32)

$99999.00(99999.00)$
RESIS.

5.16

3.28

2.71

3.18

4.74

7.40

10.46
12.96 

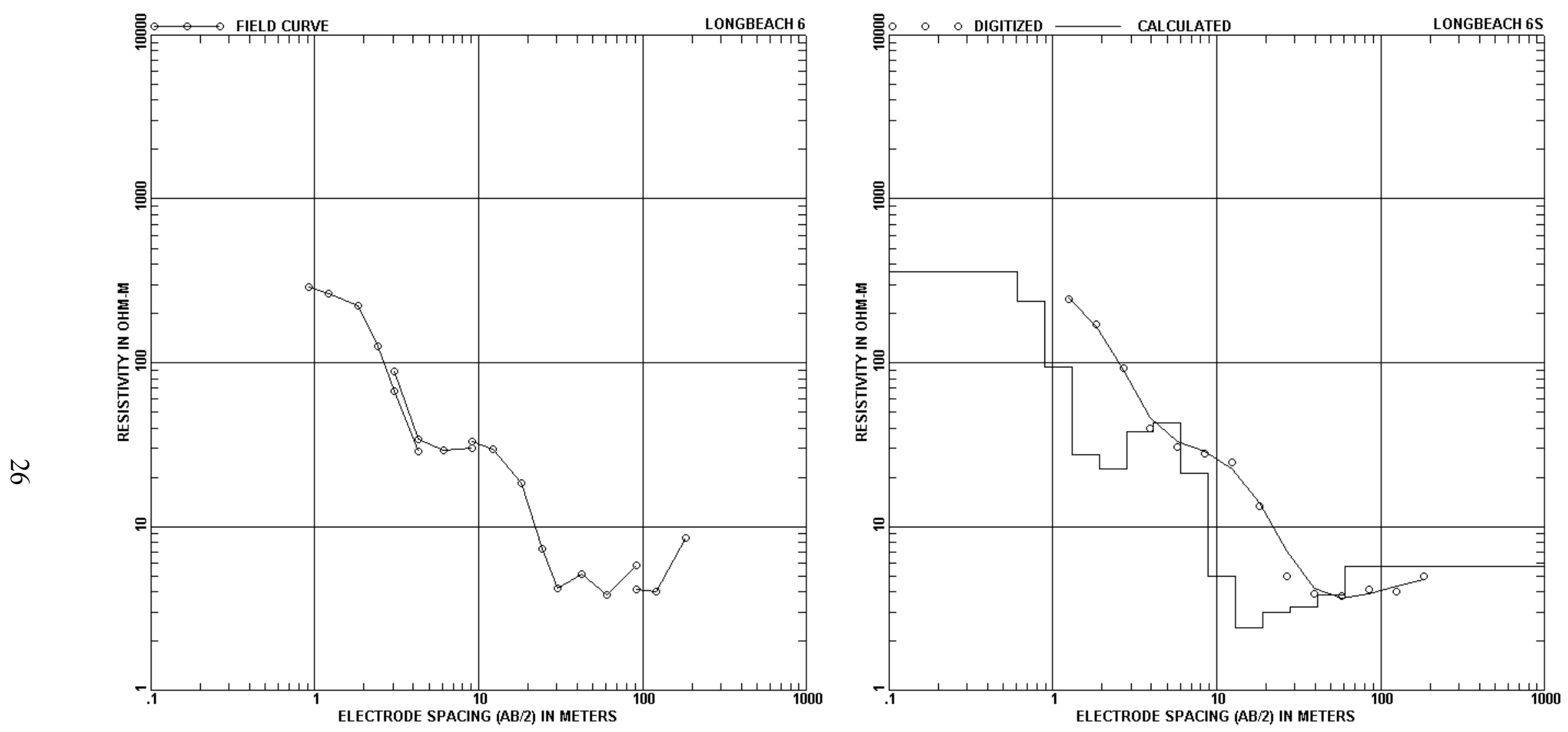

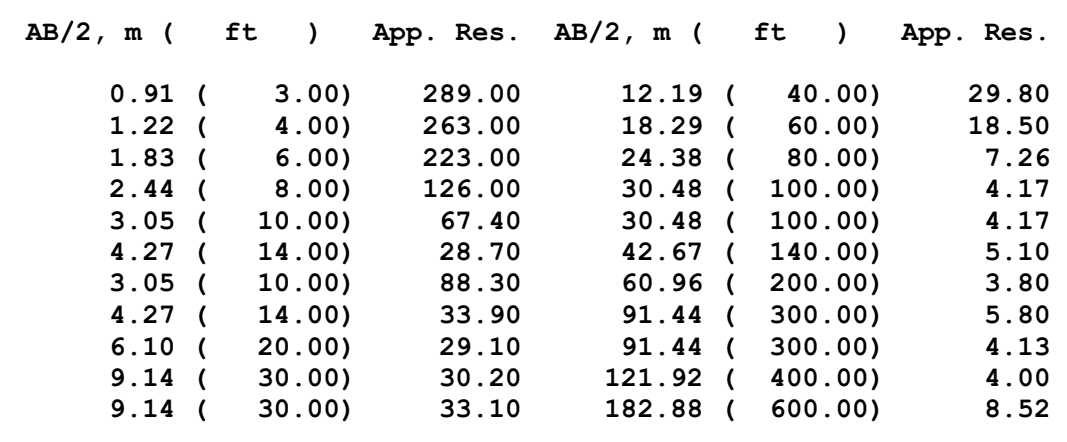

DEPTH, $m$ ( ft ) RESIS, DEPTH $m$ ( ft

\begin{tabular}{|c|c|c|c|c|c|}
\hline 0.61 & 1.99) & 356.57 & 8.89 & $29.16)$ & 21.12 \\
\hline 0.89 & $2.92)$ & 236.30 & 13.05 & $42.80)$ & 4.97 \\
\hline 1.30 & $4.28)$ & 93.84 & 19.15 & $62.82)$ & 2.40 \\
\hline 1.91 & $6.28)$ & 27.29 & 28.11 & $92.21)$ & 3.01 \\
\hline 2.81 & $9.22)$ & 22.30 & 41.25 & $135.35)$ & 3.21 \\
\hline 4.13 & 13.53) & 37.95 & 60.55 & $198.66)$ & 3.8 \\
\hline 6.06 & 19.87) & 43.04 & 99999.00 & $(99999.00)$ & 5.7 \\
\hline
\end{tabular}




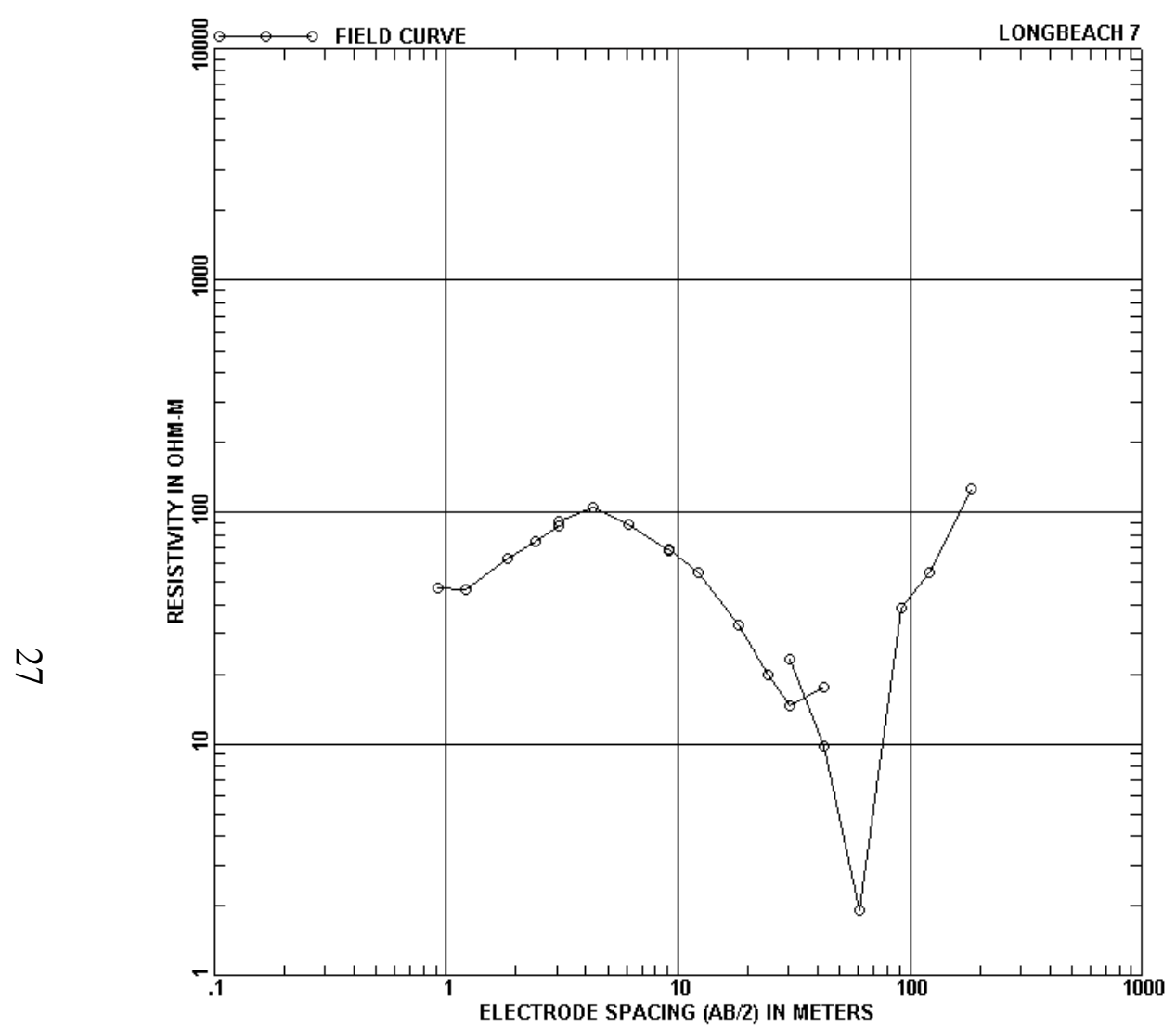

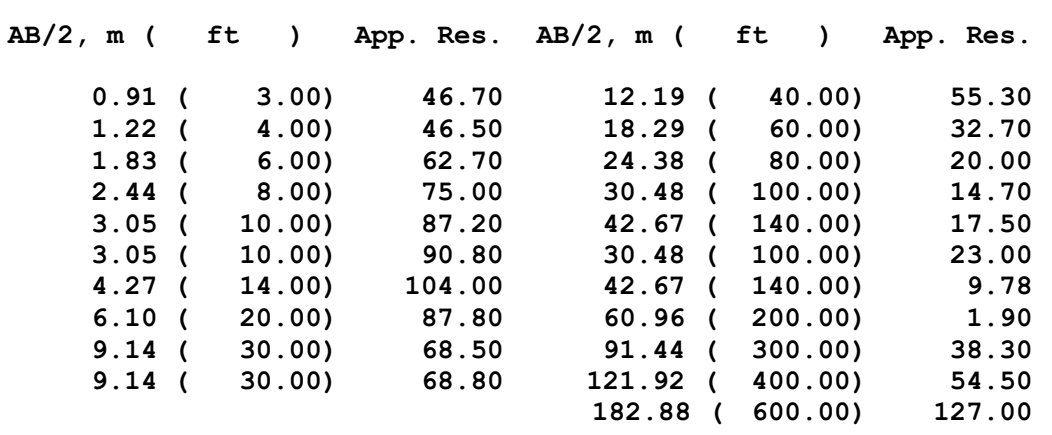



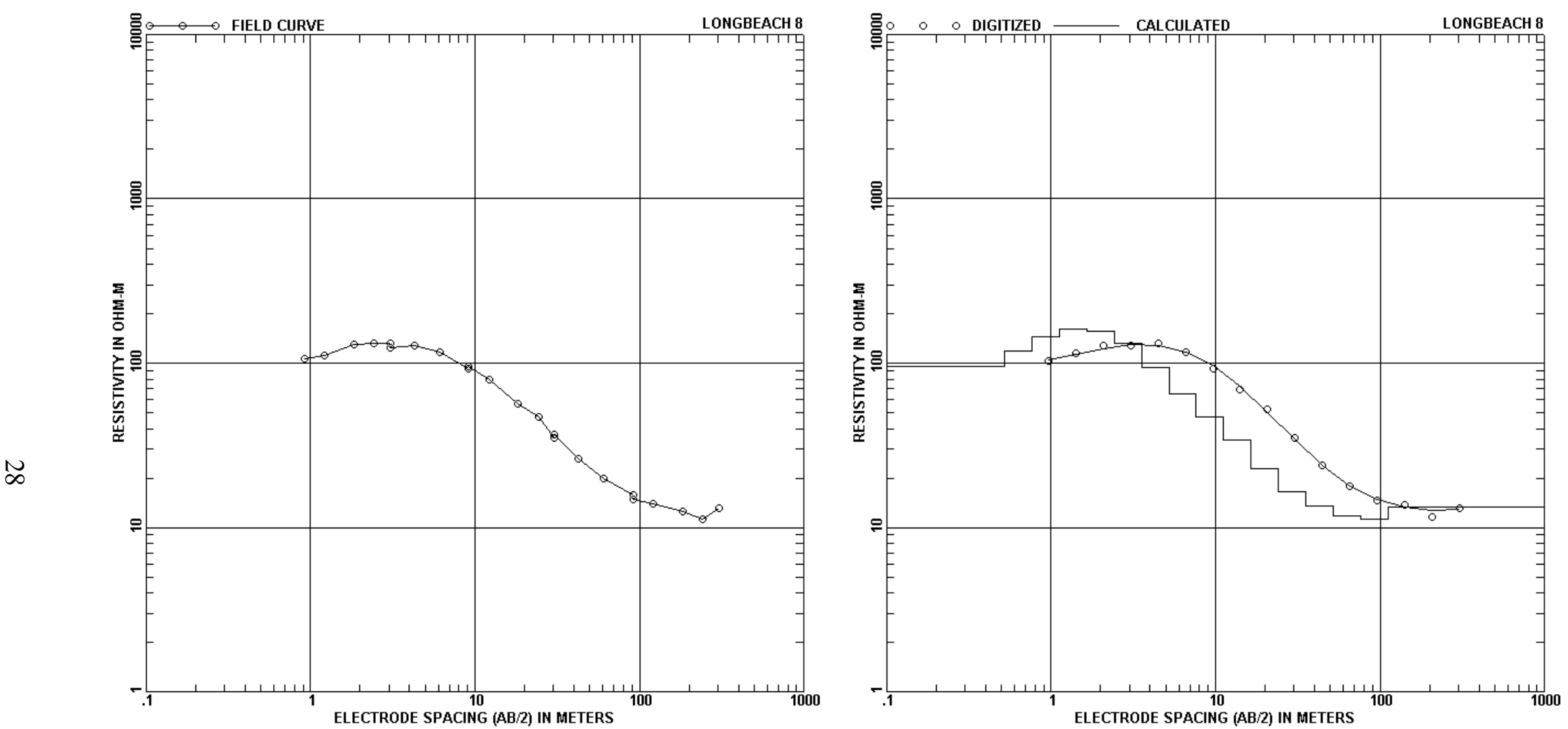

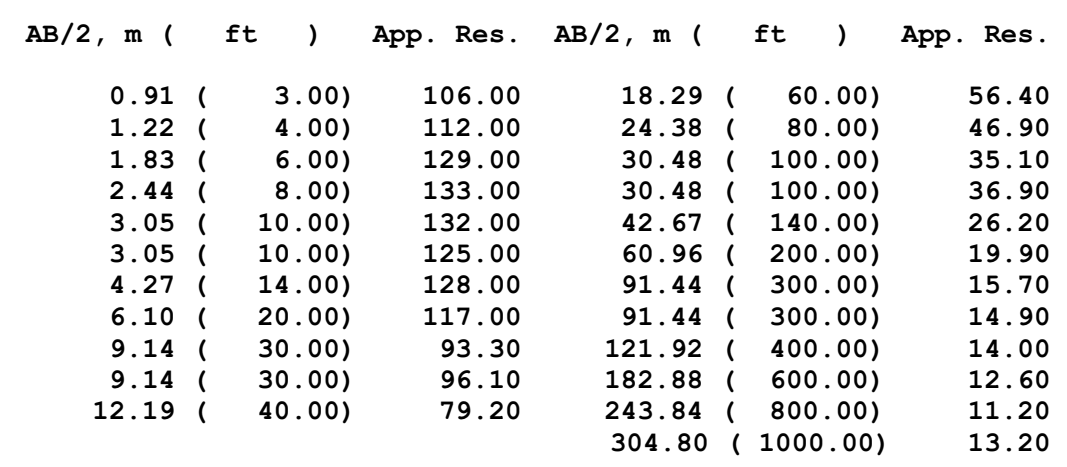

DEPTH, $m$ ( ft ) RESIS, DEPTH, m ( ft

\begin{tabular}{|c|c|c|c|c|c|}
\hline 0.52 & 1.71) & 95.34 & 11.21 & $36.79)$ & 47.07 \\
\hline 0.76 & 2.51) & 118.75 & 16.46 & $54.00)$ & 33.85 \\
\hline 1.12 & $3.68)$ & 144.31 & 24.16 & $79.26)$ & 22.77 \\
\hline 1.65 & $5.40)$ & 160.42 & 35.46 & $116.34)$ & 16.39 \\
\hline .42 & $7.93)$ & 157.37 & 52.05 & $170.76)$ & 13.52 \\
\hline 3.55 & $11.63)$ & 131.62 & 76.40 & $250.65)$ & 11.71 \\
\hline 5.20 & $17.08)$ & 94.67 & 112.14 & $367.90)$ & 11.29 \\
\hline 7.64 & $25.06)$ & 64.95 & 99999.00 & $(99999.00)$ & 13.23 \\
\hline
\end{tabular}



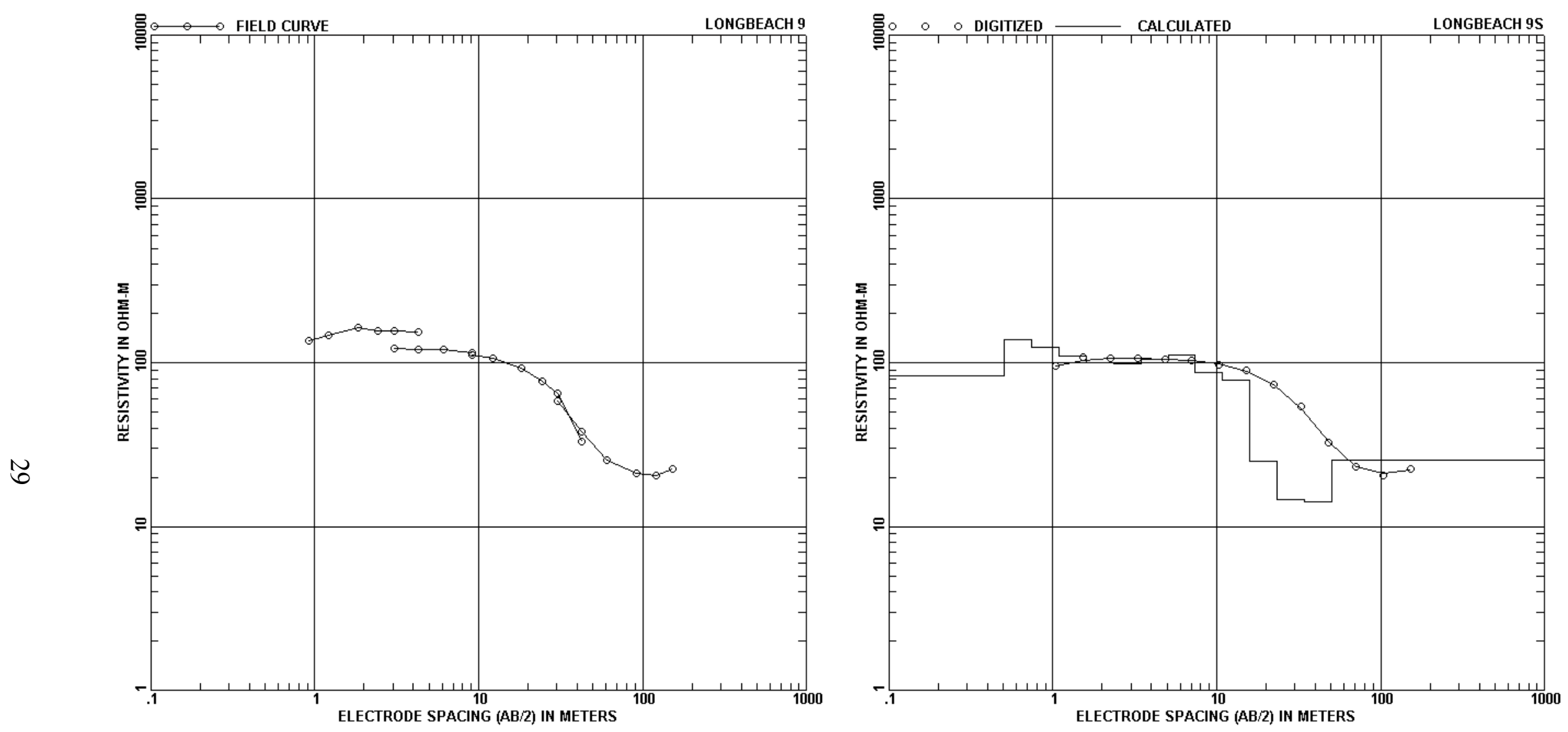

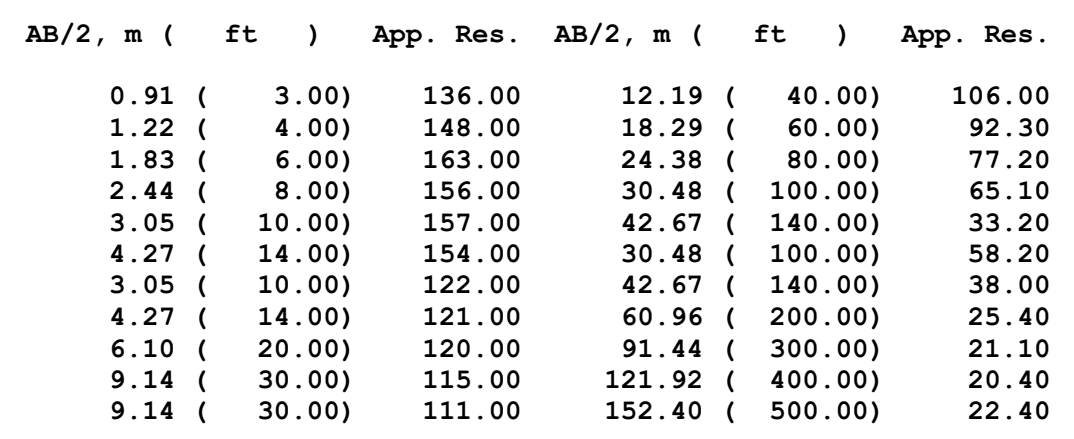

\begin{tabular}{|c|c|c|c|c|c|}
\hline DEPTH， m ( & ft, & RESIS. & DEPTH， m & ft & RESIS. \\
\hline 0.501 & $1.66)$ & 82.63 & 7.41 & $24.30)$ & 111.49 \\
\hline $0.74 i$ & $2.43)$ & 138.34 & 10.87 & $35.67)$ & 87.50 \\
\hline $1.09 i$ & $3.57)$ & 123.86 & 15.96 & $52.35)$ & 78.60 \\
\hline $1.60 i$ & $5.24)$ & 110.28 & 23.42 & $76.84)$ & 25.21 \\
\hline $2.34 i$ & $7.68)$ & 99.94 & 34.38 & ( 112.79$)$ & 14.52 \\
\hline 3.44 i & $11.28)$ & 98.40 & 50.46 & $(165.55)$ & 14.09 \\
\hline $5.05 i$ & $16.56)$ & 104.52 & 99999.00 & $(99999.00)$ & 25.26 \\
\hline
\end{tabular}



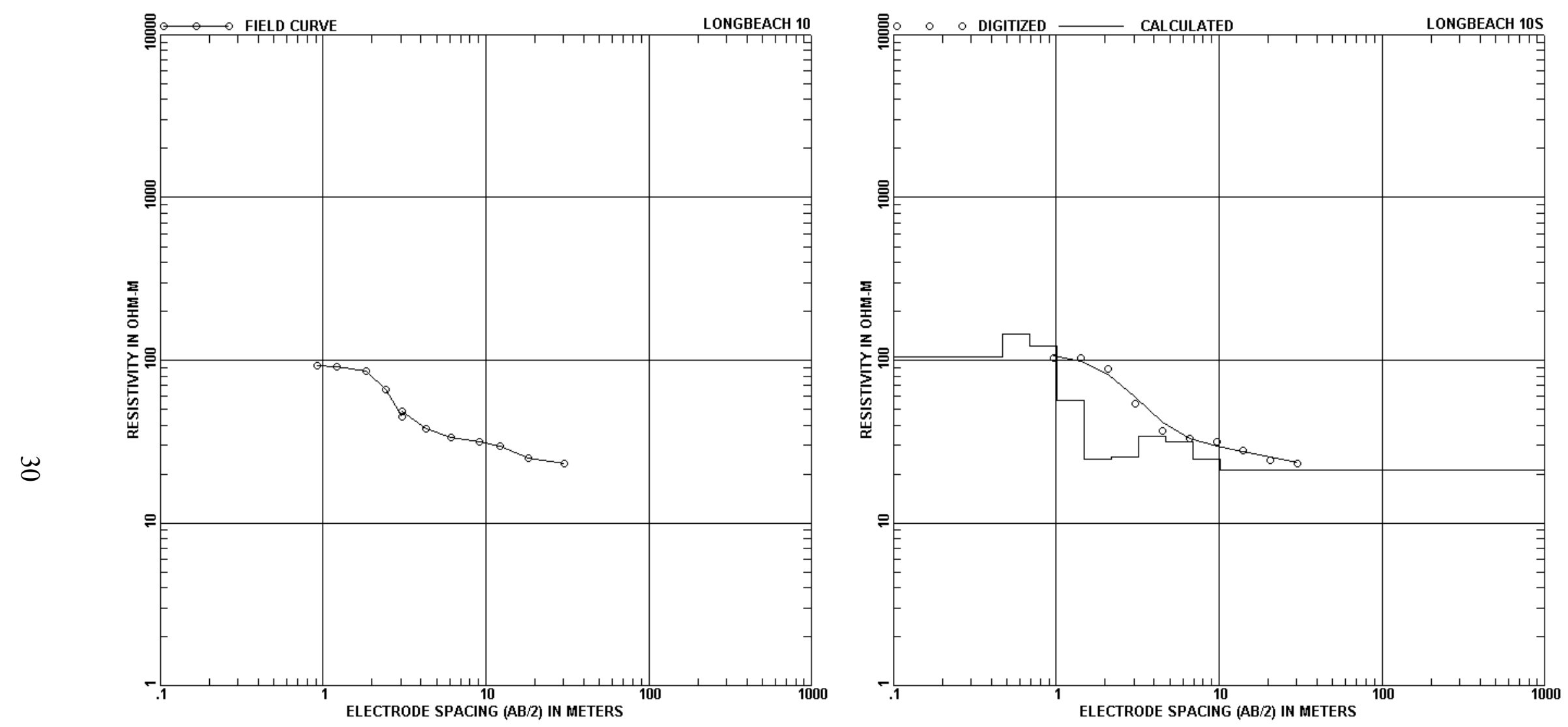

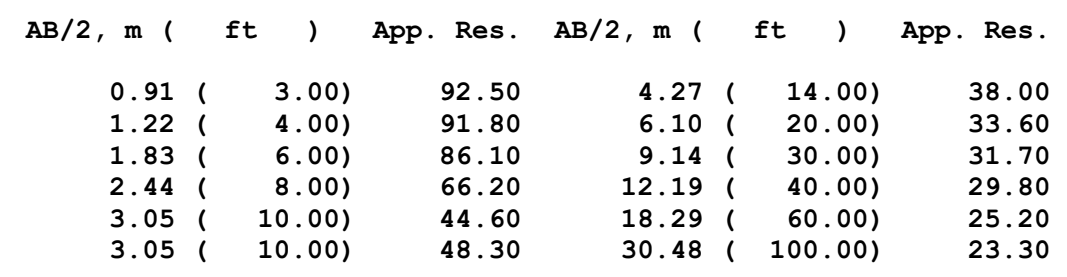

\begin{tabular}{rrrrrr} 
DEPTH, m ( & ft ) & RESIS. & DEPTH, m ( ft ) & \multicolumn{1}{l}{ RESIS. } \\
0.47( & $1.54)$ & 105.09 & 3.19( & $10.47)$ & 25.30 \\
0.69( & $2.26)$ & 144.11 & 4.68( & $15.37)$ & 33.96 \\
1.01( & $3.31)$ & 123.03 & 6.88( & $22.56)$ & 31.52 \\
1.48( & $4.86)$ & 56.48 & $10.09(133.11)$ & 24.55 \\
2.17( & $7.13)$ & 24.77 & $99999.00(99999.00)$ & 21.09
\end{tabular}



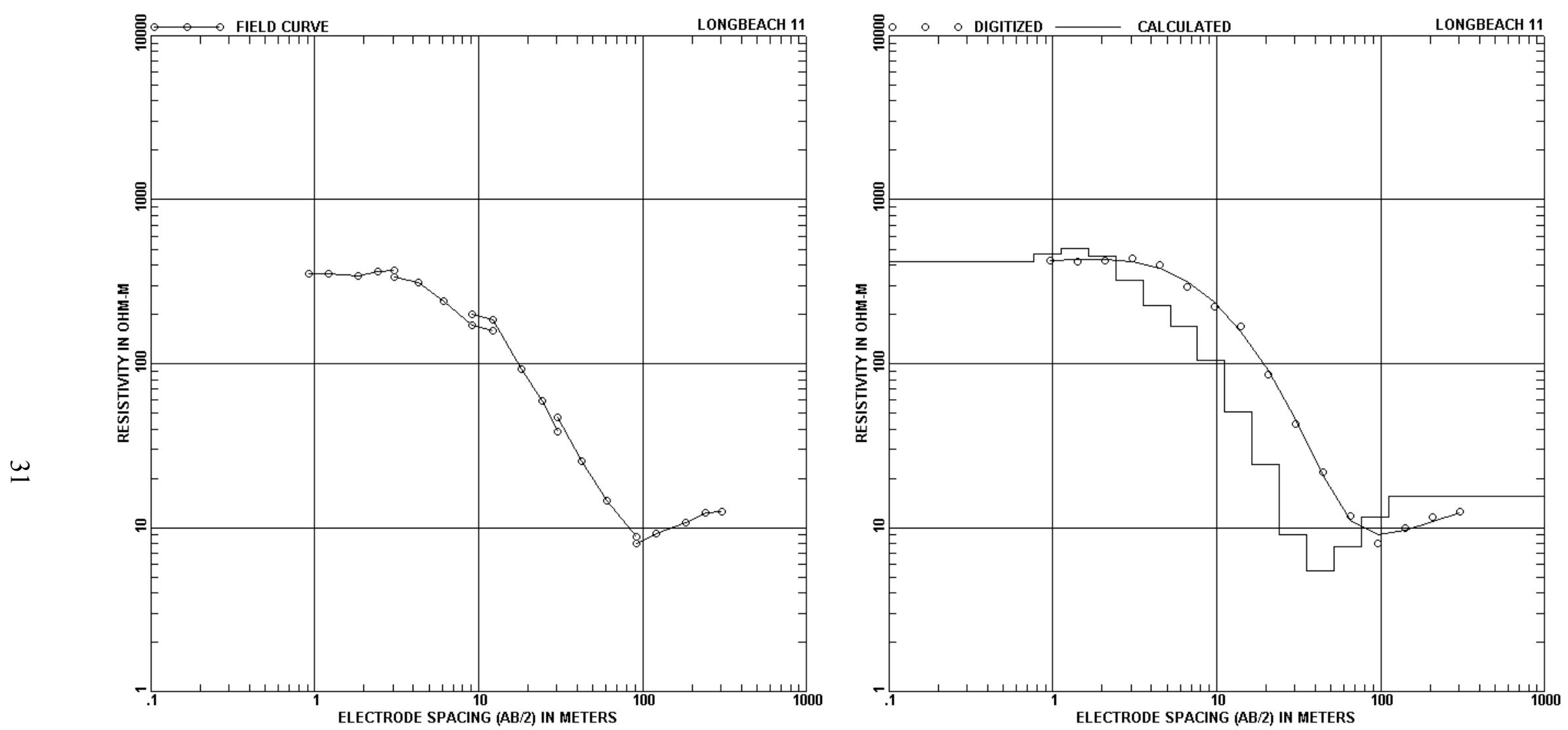

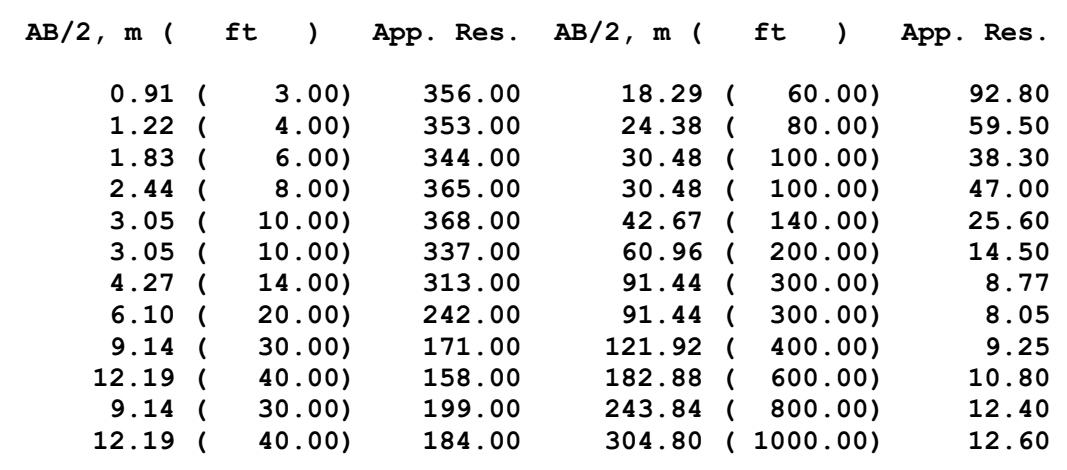

DEPTH, m ( ft ) RESIS. DEPTH, m ( ft

$\begin{array}{rlr}0.52 & ( & 1.71) \\ 0.76 & ( & 2.51) \\ 1.12 & ( & 3.68) \\ 1.65 & ( & 5.40) \\ 2.42 & ( & 7.93) \\ 3.55 & ( & 11.63) \\ 5.20 & ( & 17.08) \\ 7.64 & ( & 25.06)\end{array}$

419.53

421.98

464.94

504.86

450.57

322.57

226.68

169.92
$11.21(-36.79)$

$16.46(54.00)$

$24.16(79.26)$

$35.46(116.34)$

$52.05(170.76)$

$76.40(250.65)$

$112.14(367.90)$ $99999.00 \quad(99999.00)$
RESIS.

105.01 51.20 24.35

5.44

5.44
7.67

71.60

11.60
15.54 

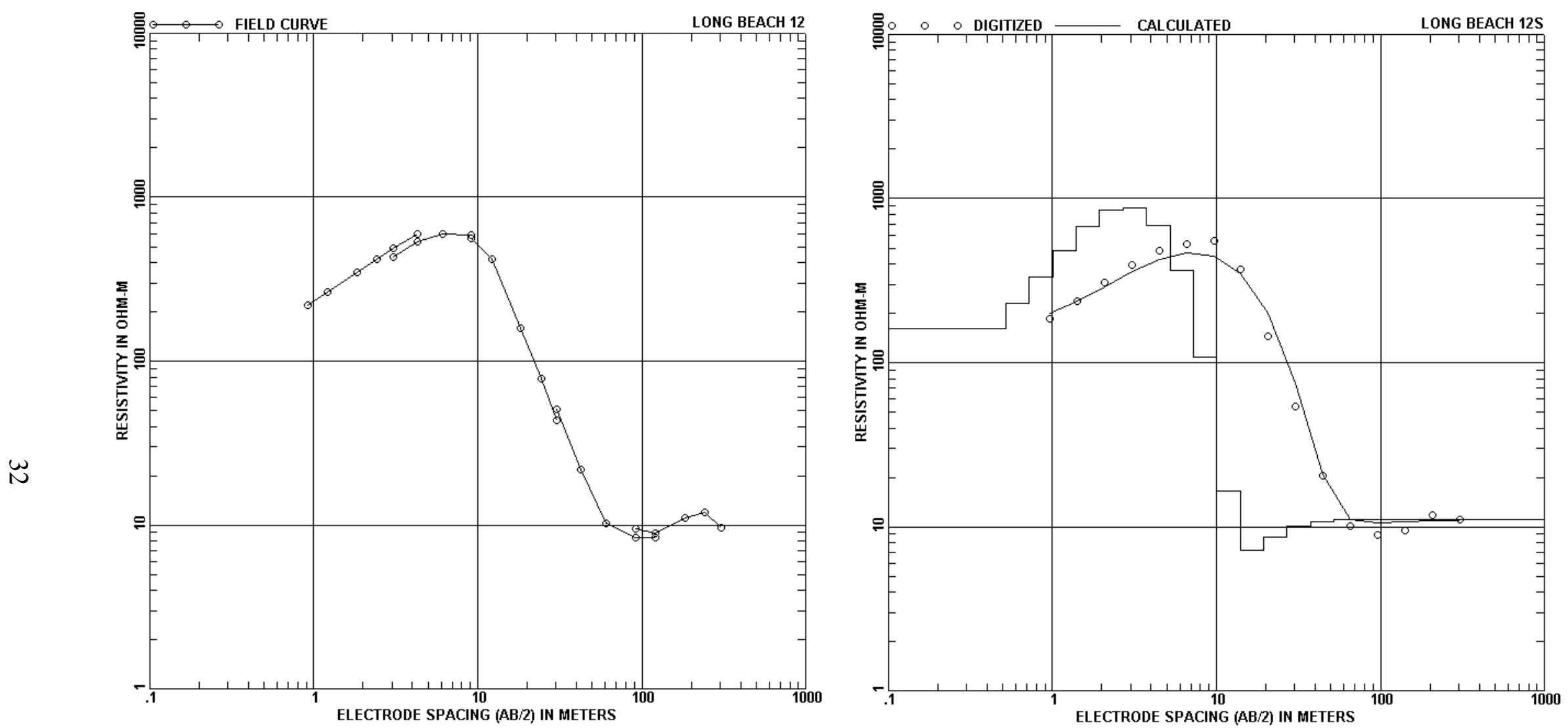

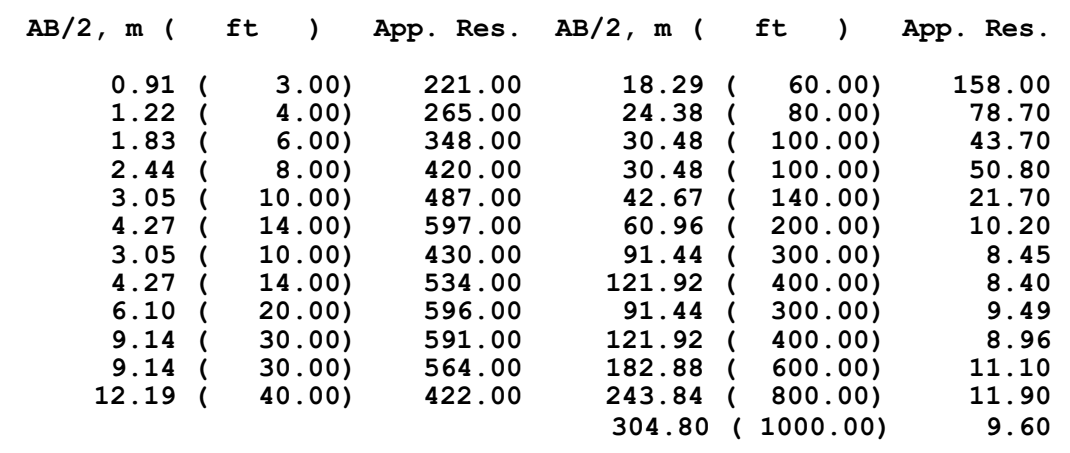

\begin{tabular}{|c|c|c|c|c|c|}
\hline DEPTH， m ( & ft ) & RESIS. & DEPTH， m & ft & RESIS. \\
\hline 0.52 & 1.71) & 162.43 & 7.23 & 23.73) & 364.82 \\
\hline 0.72 & $2.37)$ & 228.62 & 10.05 & $32.97)$ & 108.37 \\
\hline 1.00 & $3.30)$ & 334.24 & 13.96 & $45.81)$ & 16.60 \\
\hline 1.40 & $4.58)$ & 484.77 & 19.40 & $63.65)$ & 7.16 \\
\hline 1.94 & $6.37)$ & 674.02 & 26.96 & $88.45)$ & 8.60 \\
\hline 2.70 & $8.84)$ & 846.94 & 37.46 & $(122.90)$ & 10.05 \\
\hline 3.75 & $12.29)$ & 879.55 & 52.05 & $(170.76)$ & 10.73 \\
\hline 5.20 & $17.08)$ & 687.50 & 99999.00 & $(99999.00)$ & 10.99 \\
\hline
\end{tabular}



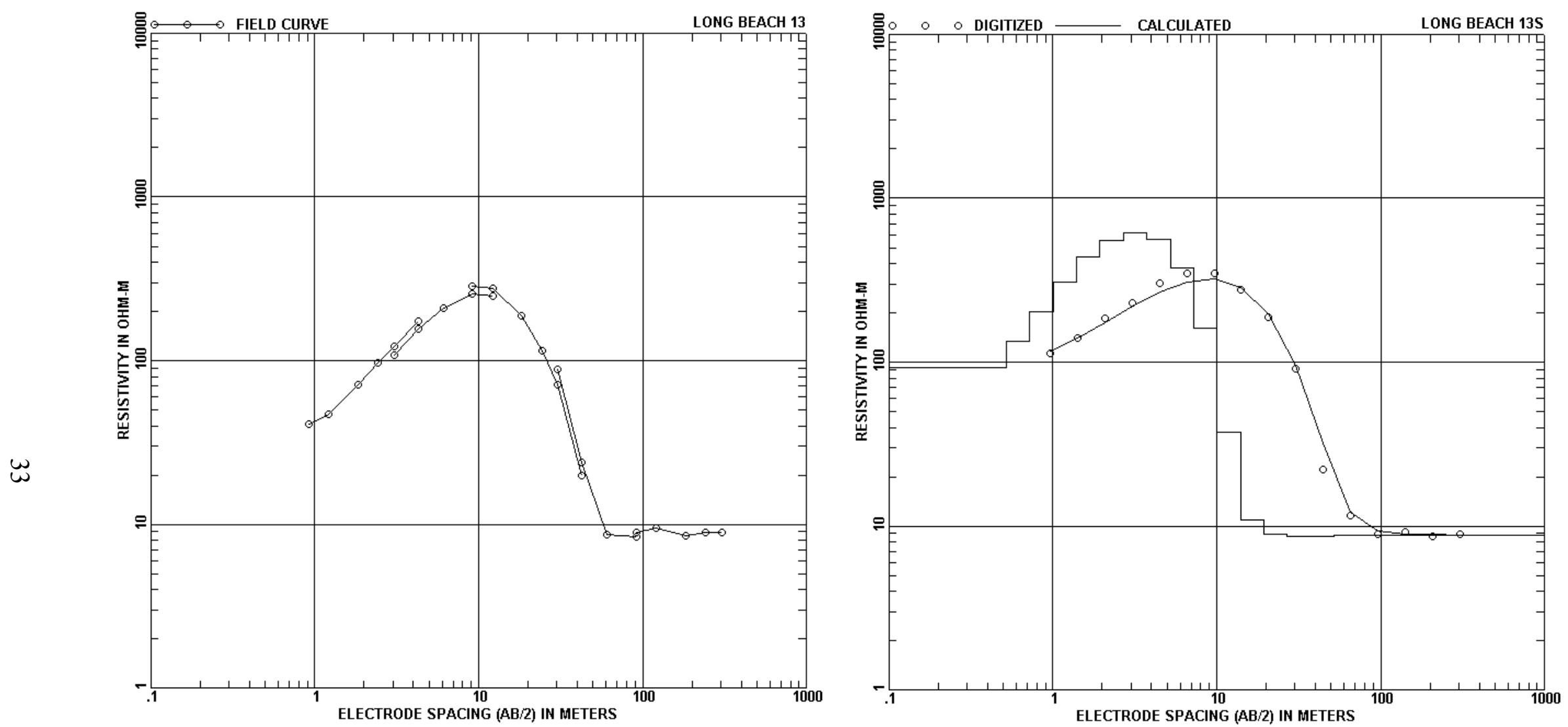

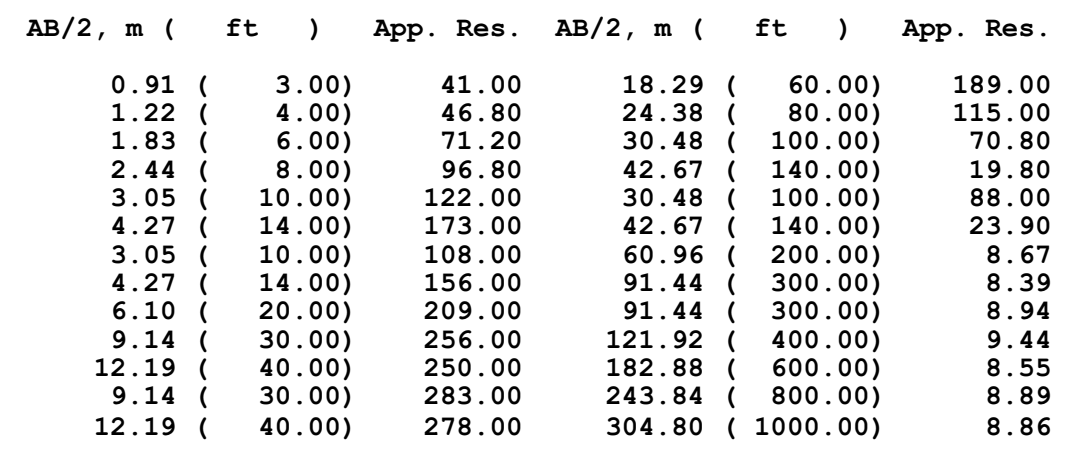

\begin{tabular}{|c|c|c|c|c|c|}
\hline DEPTH， m ( & ft ) & RESIS. & DEPTH， m & ft & RESIS. \\
\hline 0.52 & 1.71) & 92.75 & 7.23 & $23.73)$ & 378.22 \\
\hline 0.72 & $2.37)$ & 134.52 & 10.05 & $32.97)$ & 160.65 \\
\hline 1.00 & $3.30)$ & 204.63 & 13.96 & $45.81)$ & 37.12 \\
\hline 1.40 & $4.58)$ & 307.77 & 19.40 & $63.65)$ & 10.90 \\
\hline 1.94 & $6.37)$ & 435.77 & 26.96 & $88.45)$ & 8.86 \\
\hline 2.70 & $8.84)$ & 555.45 & 37.46 & $(122.90)$ & 8.61 \\
\hline 3.75 & $12.29)$ & 613.74 & 52.05 & $(170.76)$ & 8.66 \\
\hline 5.20 & $17.08)$ & 558.45 & 99999.00 & $(99999.00)$ & 8.78 \\
\hline
\end{tabular}



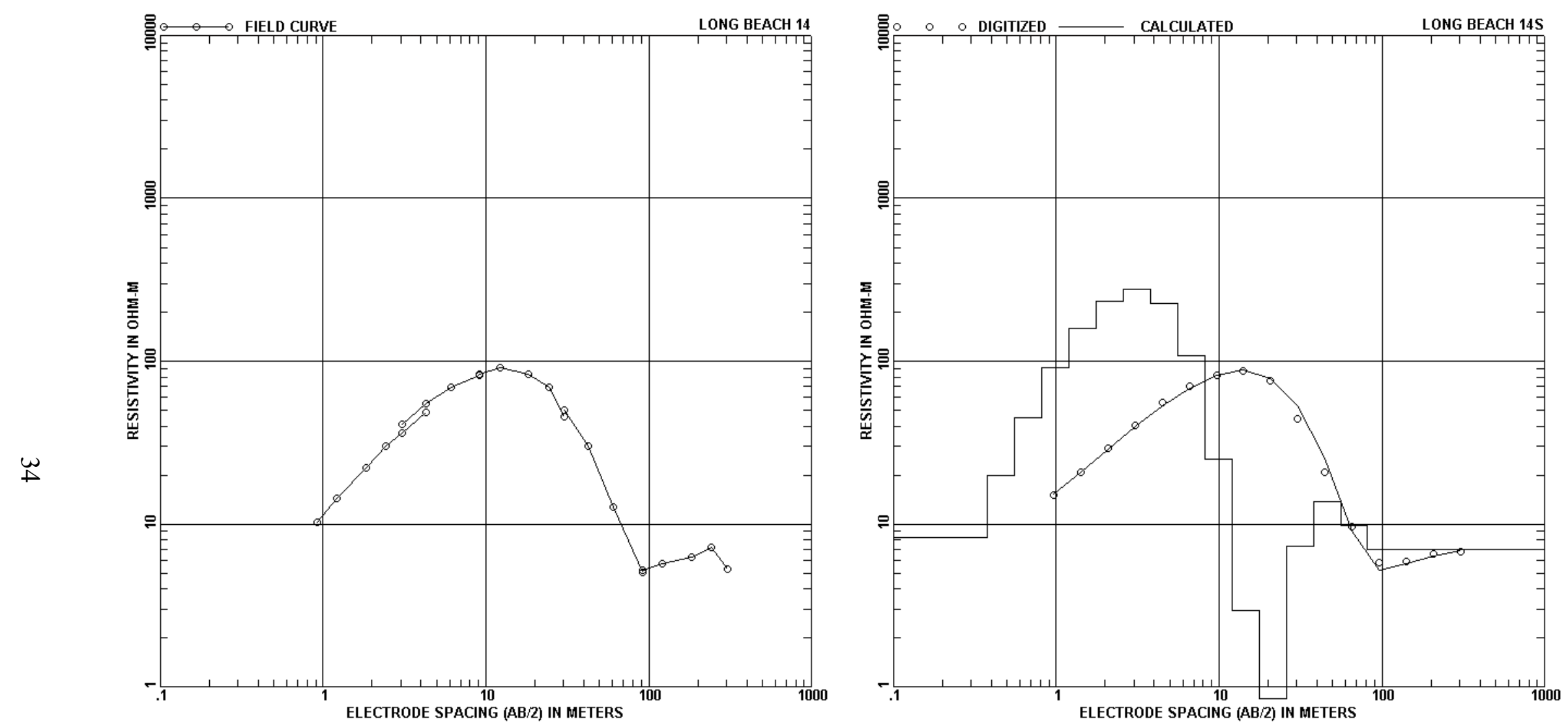

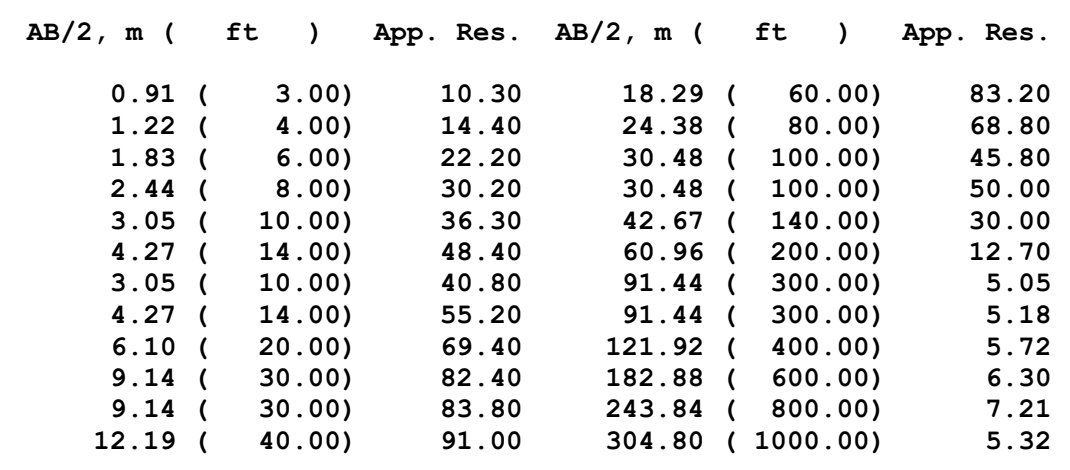

DEPTH， m ( ft )

$\begin{array}{rlr}0.38 & ( & 1.24) \\ 0.56 & ( & 1.83) \\ 0.82 & ( & 2.68) \\ 1.20 & ( & 3.94) \\ 1.76 & ( & 5.78) \\ 2.59 & ( & 8.48) \\ 3.79 & ( & 12.45) \\ 5.57 & ( & 18.27)\end{array}$
RESIS.

8.30
19.79
44.89
91.09
159.36
234.49
274.47
225.05

8
12
17
25
37
55
81
99999

RESIS.

\begin{tabular}{|c|c|c|}
\hline 8.17 & $26.82)$ & 107.58 \\
\hline 12.00 & $39.37)$ & 25.07 \\
\hline 17.6 & $57.78)$ & 2.96 \\
\hline 25.85 & $84.81)$ & 0.84 \\
\hline 37.94 & $124.49)$ & 7.36 \\
\hline 55.69 & $182.72)$ & 13.79 \\
\hline 1.75 & $268.20)$ & 9.86 \\
\hline 90 & $9999.00)$ & 6.97 \\
\hline
\end{tabular}

07.58 2.96

0.84

13.79 6.97 

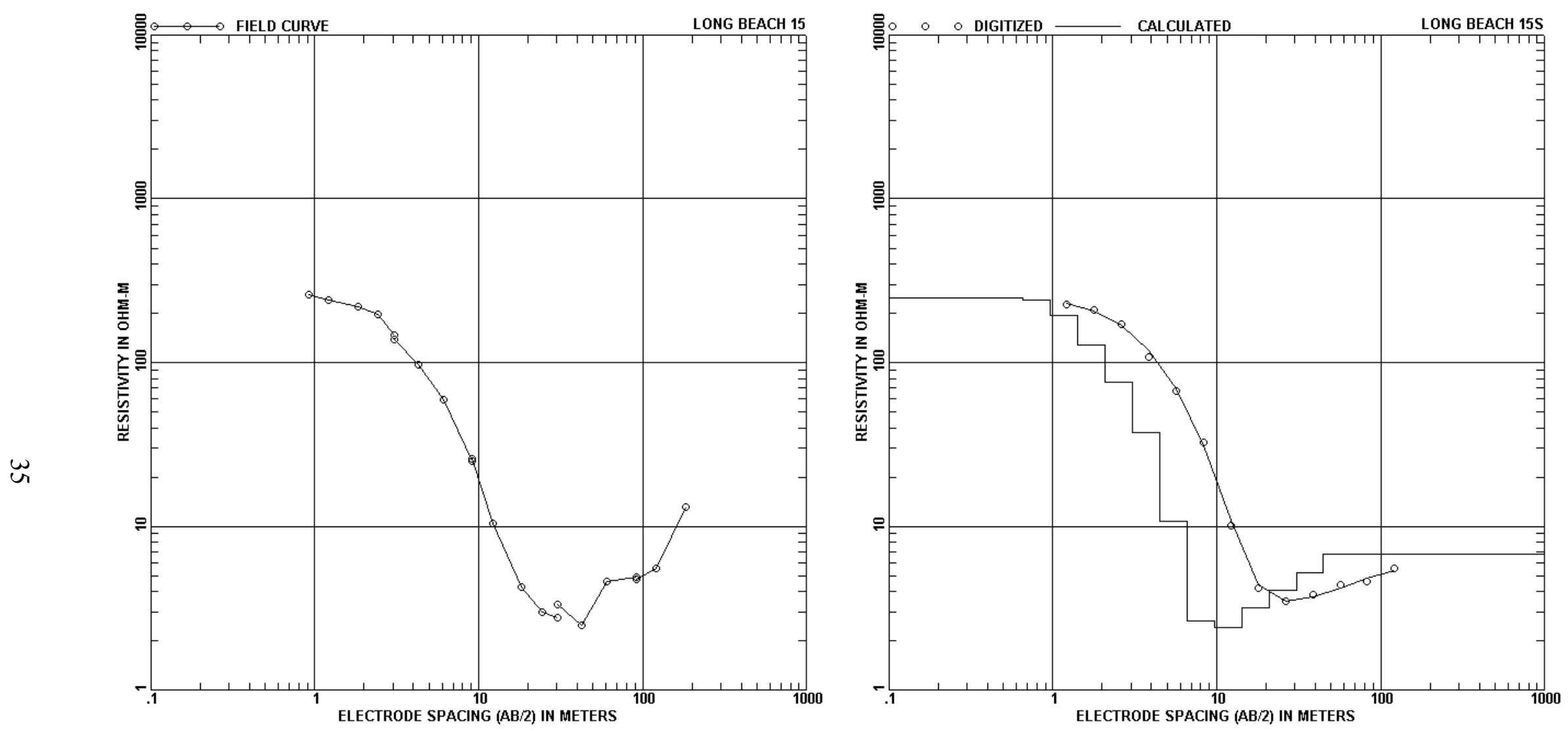

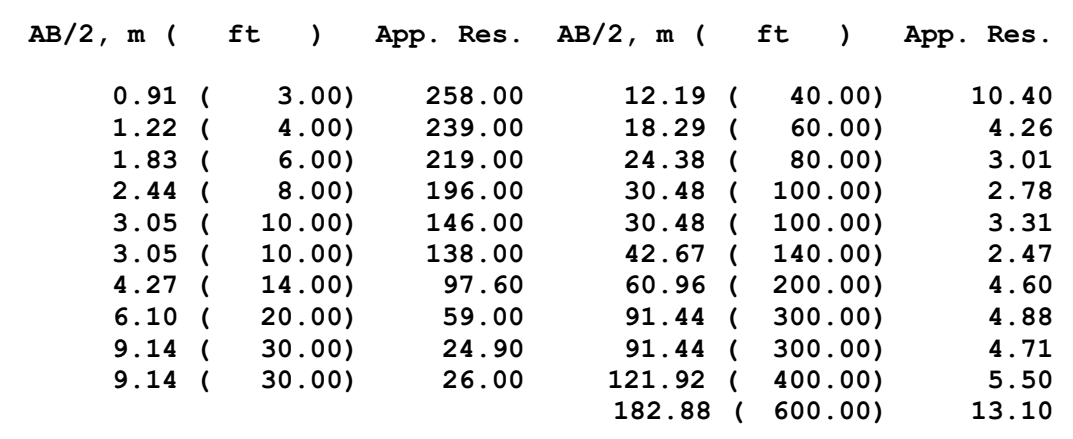

\begin{tabular}{|c|c|c|c|c|c|}
\hline DEPTH， m ( & ft & RESIS. & DEPTH， m ( & ft & RESIS. \\
\hline 0.661 & $2.16)$ & 248.80 & 6.58 & $21.60)$ & 10.69 \\
\hline $0.97 i$ & $3.17)$ & 240.51 & 9.66 & $31.70)$ & 2.65 \\
\hline $1.42 \%$ & $4.65)$ & 193.78 & 14.18 & $46.54)$ & 2.40 \\
\hline $2.08 i$ & $6.83)$ & 127.81 & 20.82 & $68.31)$ & 3.19 \\
\hline $3.06 i$ & $10.03)$ & 75.58 & 30.56 & $100.26)$ & 4.07 \\
\hline 4.49 i & $14.72)$ & 37.46 & 44.85 & $147.16)$ & 5.20 \\
\hline & & & 99999.00 & $(99999.00)$ & 6.75 \\
\hline
\end{tabular}



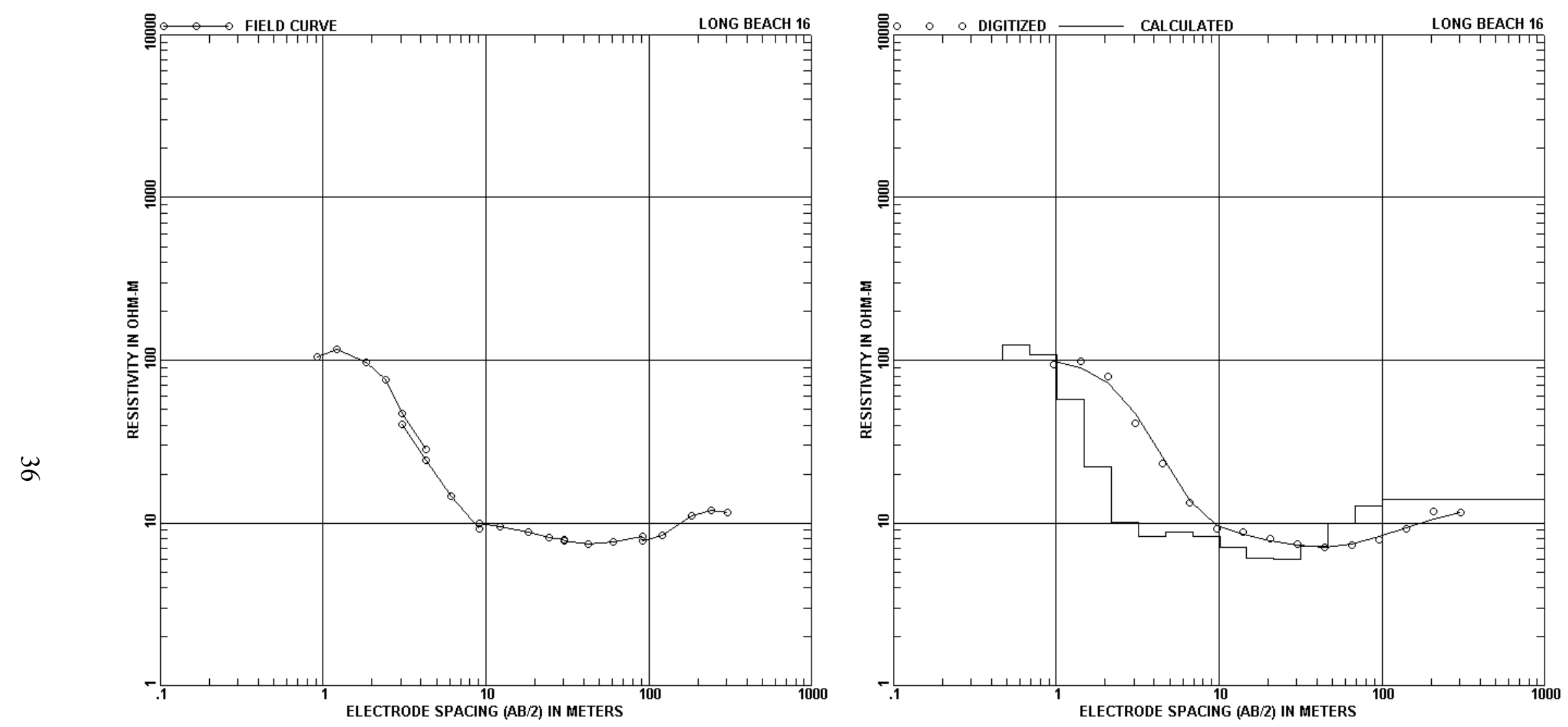

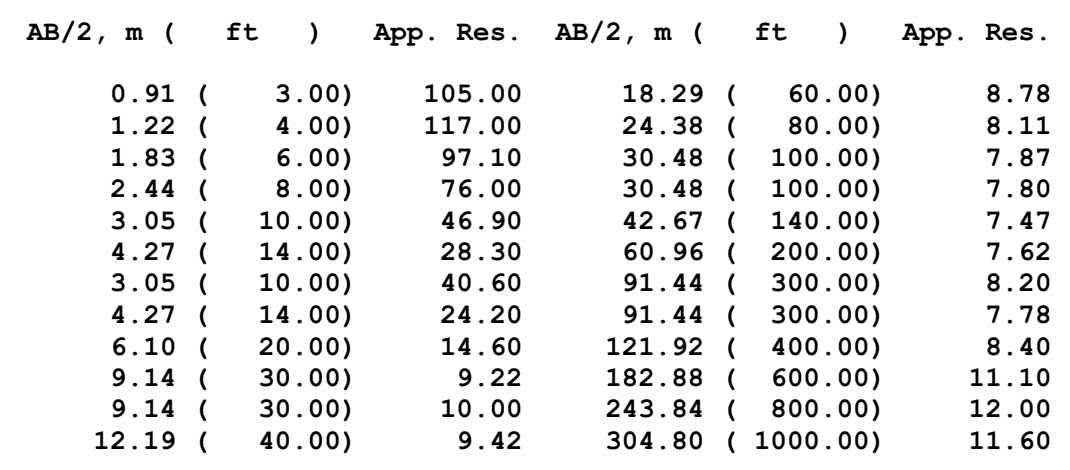

DEPTH, $m$ ( ft ) RESIS. DEPTH, $m($ ft

\begin{tabular}{|c|c|c|c|c|c|}
\hline 0.47 & $1.54)$ & 99.95 & 10.09 & $33.11)$ & 8.32 \\
\hline 0.69 & $2.26)$ & 124.58 & 14.81 & $48.60)$ & 7.10 \\
\hline 1.01 & $3.31)$ & 108.05 & 21.74 & $71.34)$ & 6.08 \\
\hline 1.48 & $4.86)$ & 57.89 & 31.91 & $104.71)$ & 5.97 \\
\hline 2.17 & $7.13)$ & 22.02 & 46.84 & $153.69)$ & 7.23 \\
\hline 3.19 & $10.47)$ & 10.03 & 68.76 & $225.58)$ & 9.88 \\
\hline 4.68 & $15.37)$ & 8.30 & 100.92 & $331.11)$ & 12.64 \\
\hline 6.88 & $22.56)$ & 8.74 & 99999.00 & $(99999.00)$ & 14.0 \\
\hline
\end{tabular}



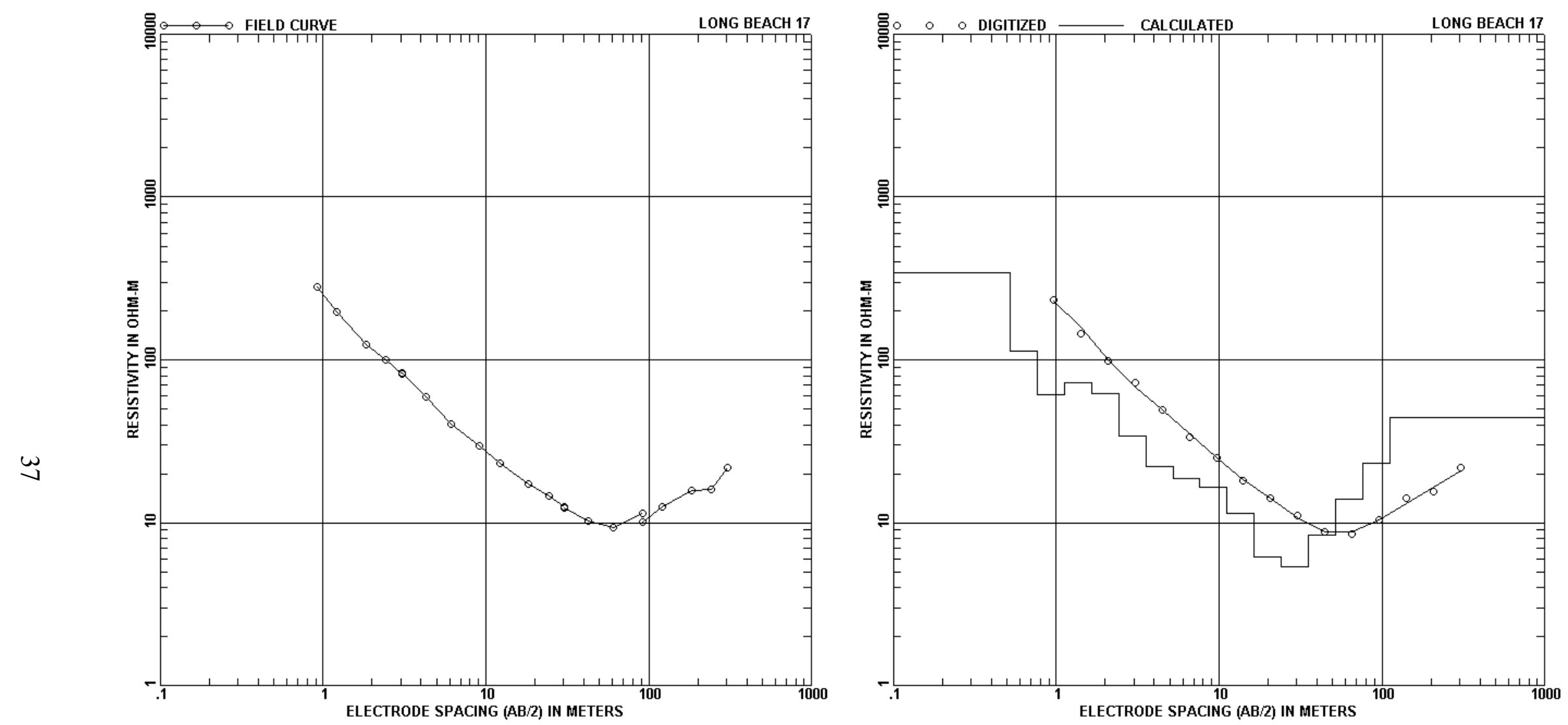

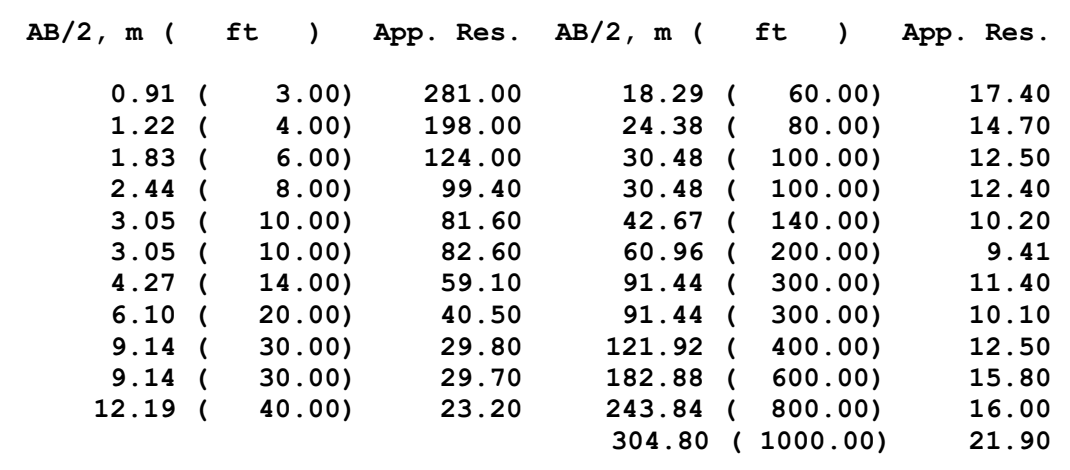

DEPTH, $m$ ( ft ) RESIS, DEPTH, $m($ ft

\begin{tabular}{|c|c|c|c|c|c|}
\hline 0.52 & 1.71) & 343.57 & 11.21 & $36.79)$ & 16.52 \\
\hline 0.76 & $2.51)$ & 113.44 & 16.46 & $54.00)$ & 11.46 \\
\hline 1.12 & $3.68)$ & 61.01 & 24.16 & $79.26)$ & 6.15 \\
\hline 1.65 & $5.40)$ & 72.10 & 35.46 & $116.34)$ & 5.36 \\
\hline 2.42 & $7.93)$ & 62.22 & 52.05 & $170.76)$ & 8.44 \\
\hline 3.55 & $11.63)$ & 34.02 & 76.40 & $250.65)$ & 13.97 \\
\hline 5.20 & $17.08)$ & 22.25 & 112.14 & $367.90)$ & 23.30 \\
\hline 7.64 & $25.06)$ & 18.69 & 9999.00 & $(99999.00)$ & 44.31 \\
\hline
\end{tabular}



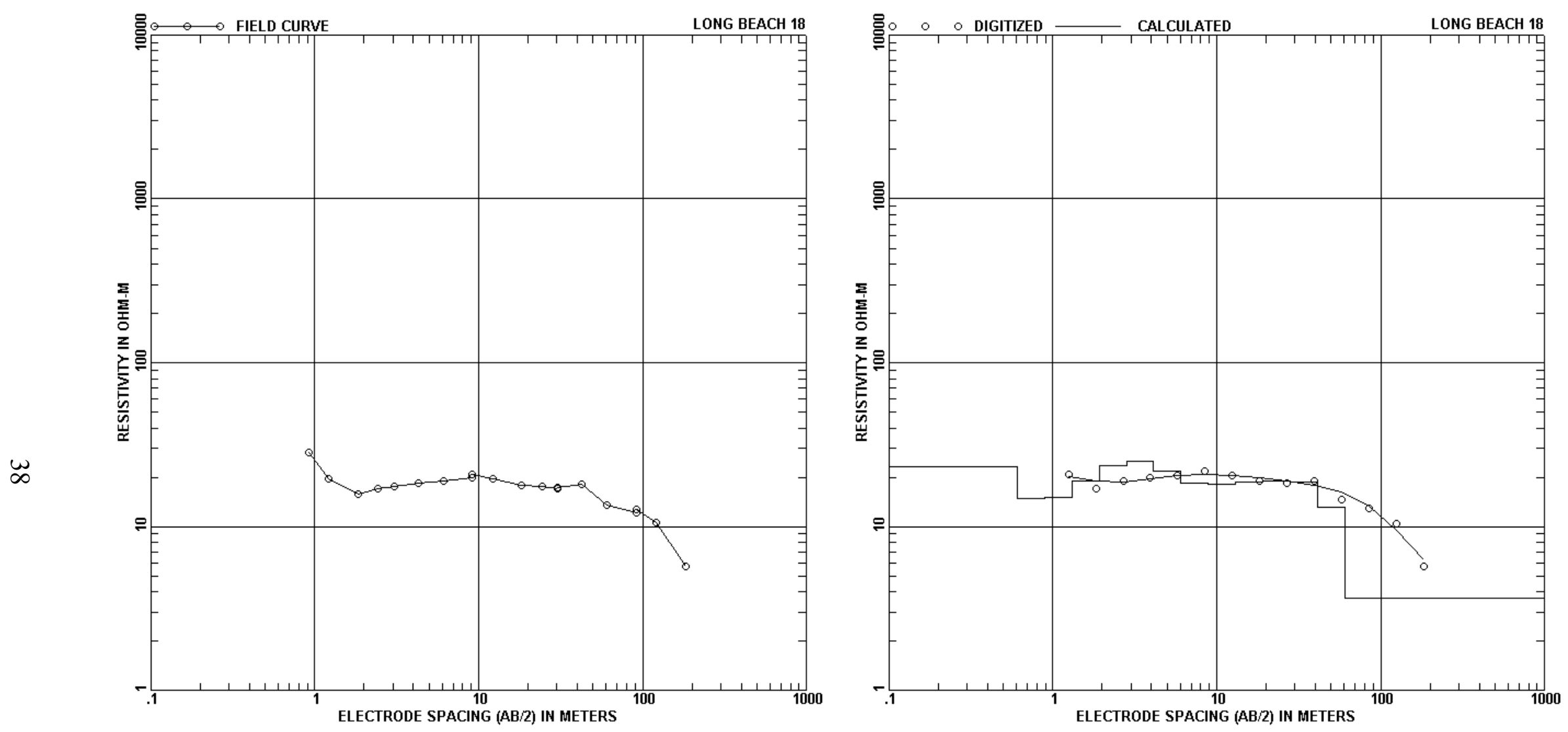

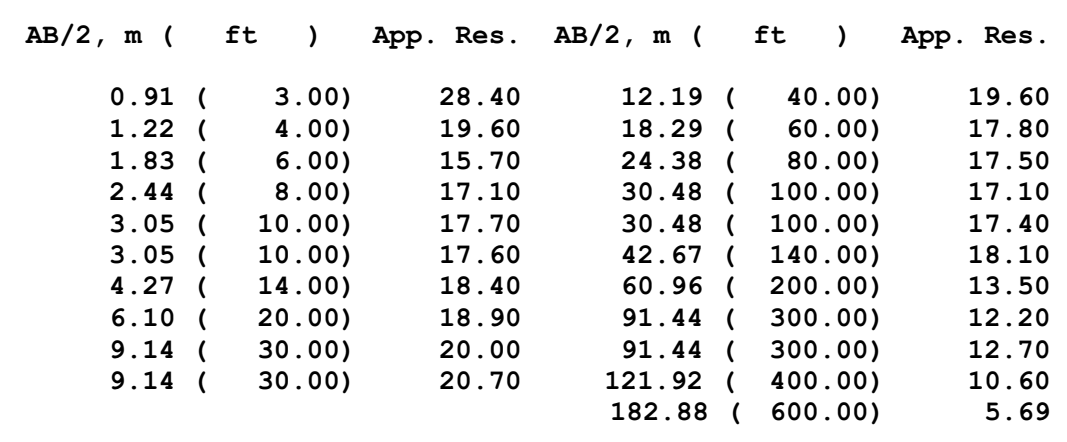

DEPTH, $m$ ( ft ) RESIS, DEPTH, m ( ft

\begin{tabular}{|c|c|}
\hline 0.61 & \\
\hline 0.89 & \\
\hline 1.30 & \\
\hline 1.91 & \\
\hline 2.81 & \\
\hline 4.13 & \\
\hline 6.06 & \\
\hline
\end{tabular}

23.13

14.85

15.08

19.09

23.41

24.87

21.77
$8.89(29.16)$

$13.05(42.80)$

$19.15(62.82)$

28.11 ( 92.21)

41.25 ( 135.35)

$60.55(198.66)$

$99999.00(99999.00)$
RESIS.

18.35 17.99 18.78

18.89

18.75

13.18 

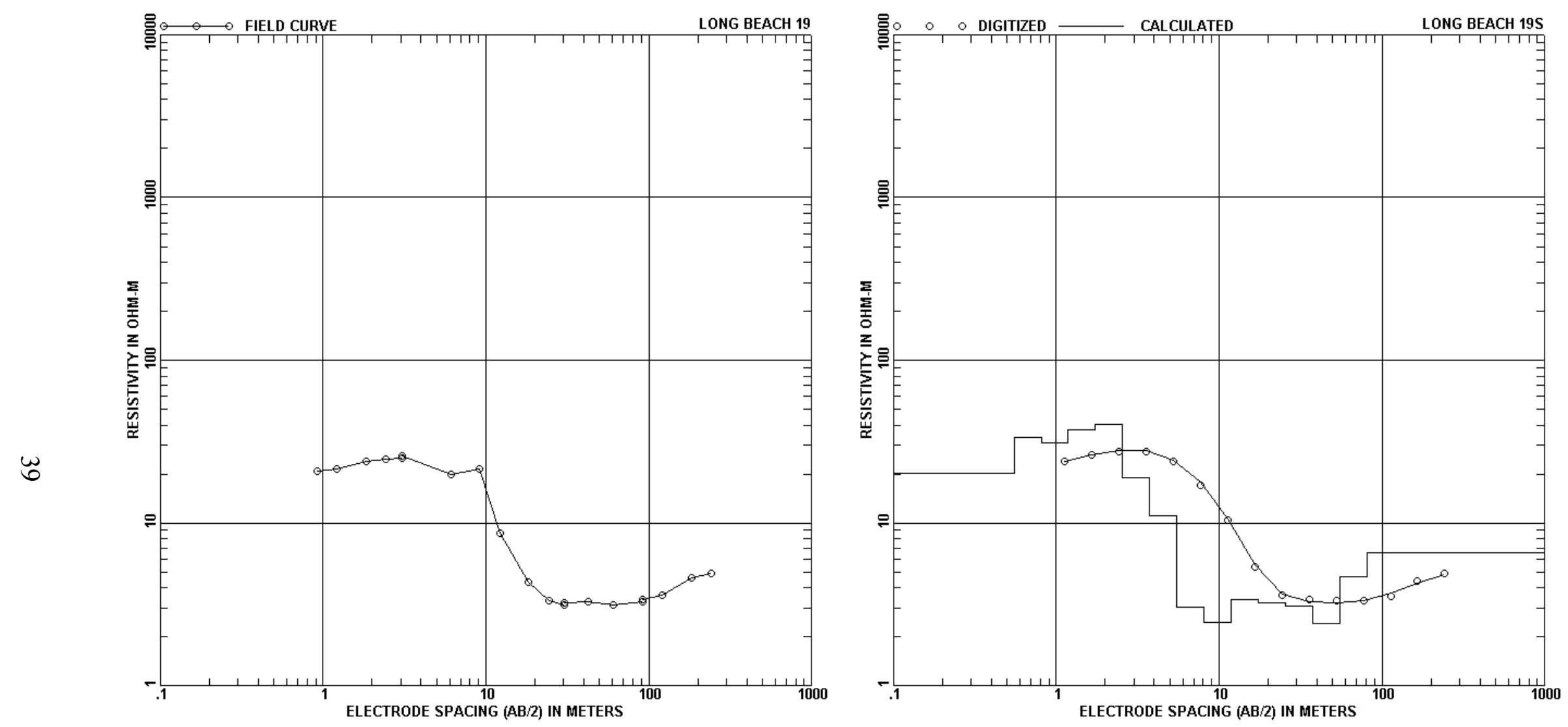

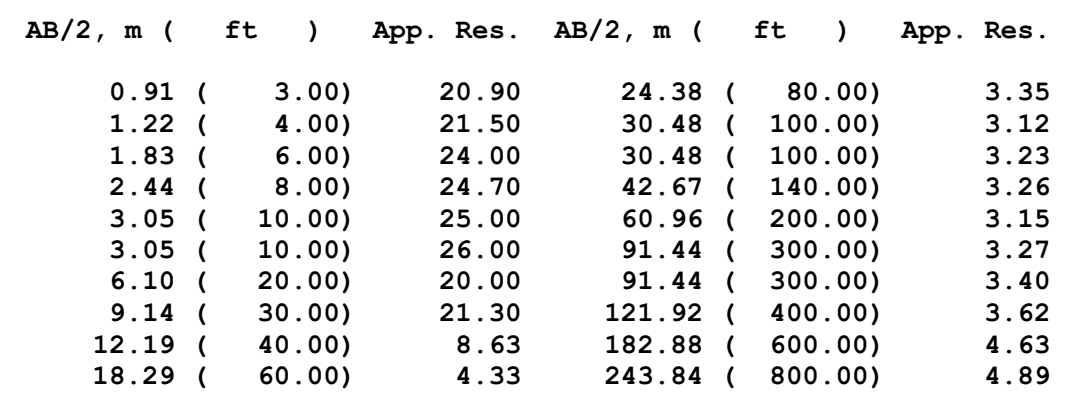

\begin{tabular}{|c|c|c|c|c|c|}
\hline DEPTH， m ( & ft & RESIS. & DEPTH， m & ft & RESIS. \\
\hline 0.551 & $1.80)$ & 20.32 & 8.07 & $26.49)$ & 3.05 \\
\hline 0.81( & $2.65)$ & 33.52 & 11.85 & $38.88)$ & 2.46 \\
\hline 1.19 ( & $3.89)$ & 31.00 & 17.39 & $57.07)$ & 3.38 \\
\hline $1.74 （$ & $5.71)$ & 37.49 & 25.53 & $83.76)$ & 3.24 \\
\hline 2.551 & $8.38)$ & 40.23 & 37.47 & $122.95)$ & 3.08 \\
\hline $3.75 i$ & $12.29)$ & 18.91 & 55.01 & ( 180.47$)$ & 2.39 \\
\hline $5.50 i$ & $18.05)$ & 11.05 & 80.74 & ( 264.89) & 4.68 \\
\hline & & & 99999.00 & $(99999.00)$ & 6.54 \\
\hline
\end{tabular}



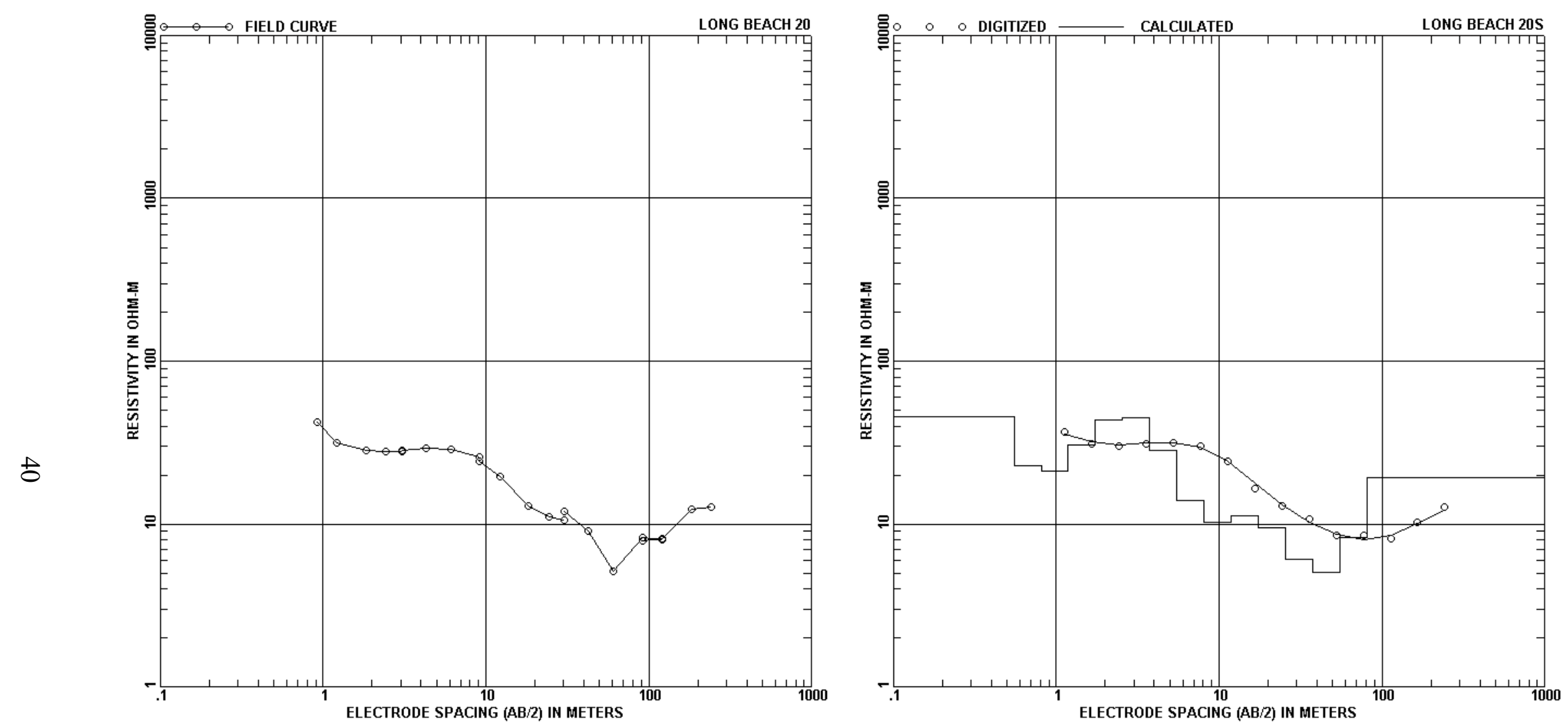

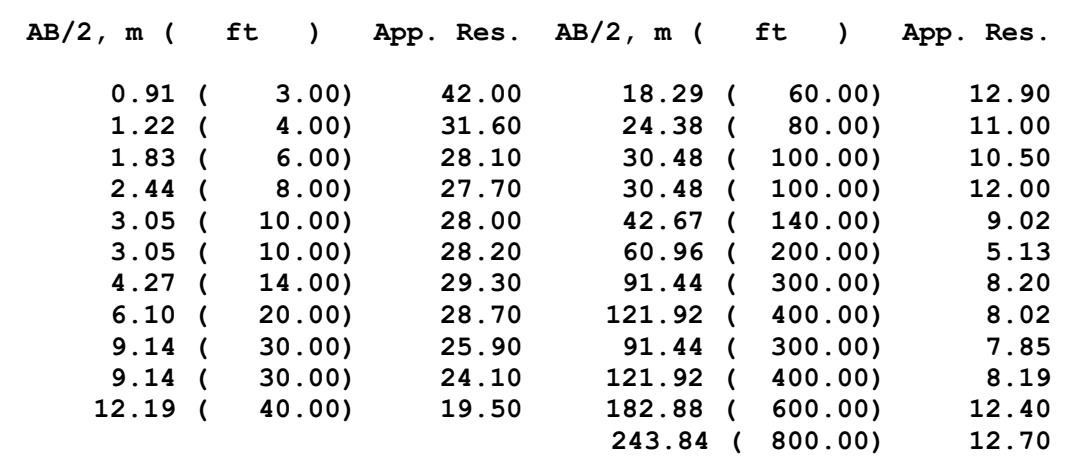

DEPTH， m ( ft )

$\begin{array}{rlr}0.55 & ( & 1.80) \\ 0.81 & ( & 2.65) \\ 1.19 & ( & 3.89) \\ 1.74 & ( & 5.71) \\ 2.55 & ( & 8.38) \\ 3.75 & ( & 12.29) \\ 5.50 & ( & 18.05)\end{array}$

\begin{abstract}
RESIS.
\end{abstract}
45.40
22.86

22.86

21.04

30.56

43.80

45.20

28.51
DEPTH, $m$ ( ft )

\begin{tabular}{|c|c|c|}
\hline 8.07 & $26.49)$ & 13.93 \\
\hline 11.85 & $38.88)$ & 10.30 \\
\hline 17.39 & $57.07)$ & 11.18 \\
\hline 25.53 & $83.76)$ & 9.42 \\
\hline 37.47 & $122.95)$ & 6.10 \\
\hline 55.01 & $180.47)$ & 5.06 \\
\hline 30.7 & $264.89)$ & 8.30 \\
\hline 99.00 & $999.00)$ & 19.32 \\
\hline
\end{tabular}



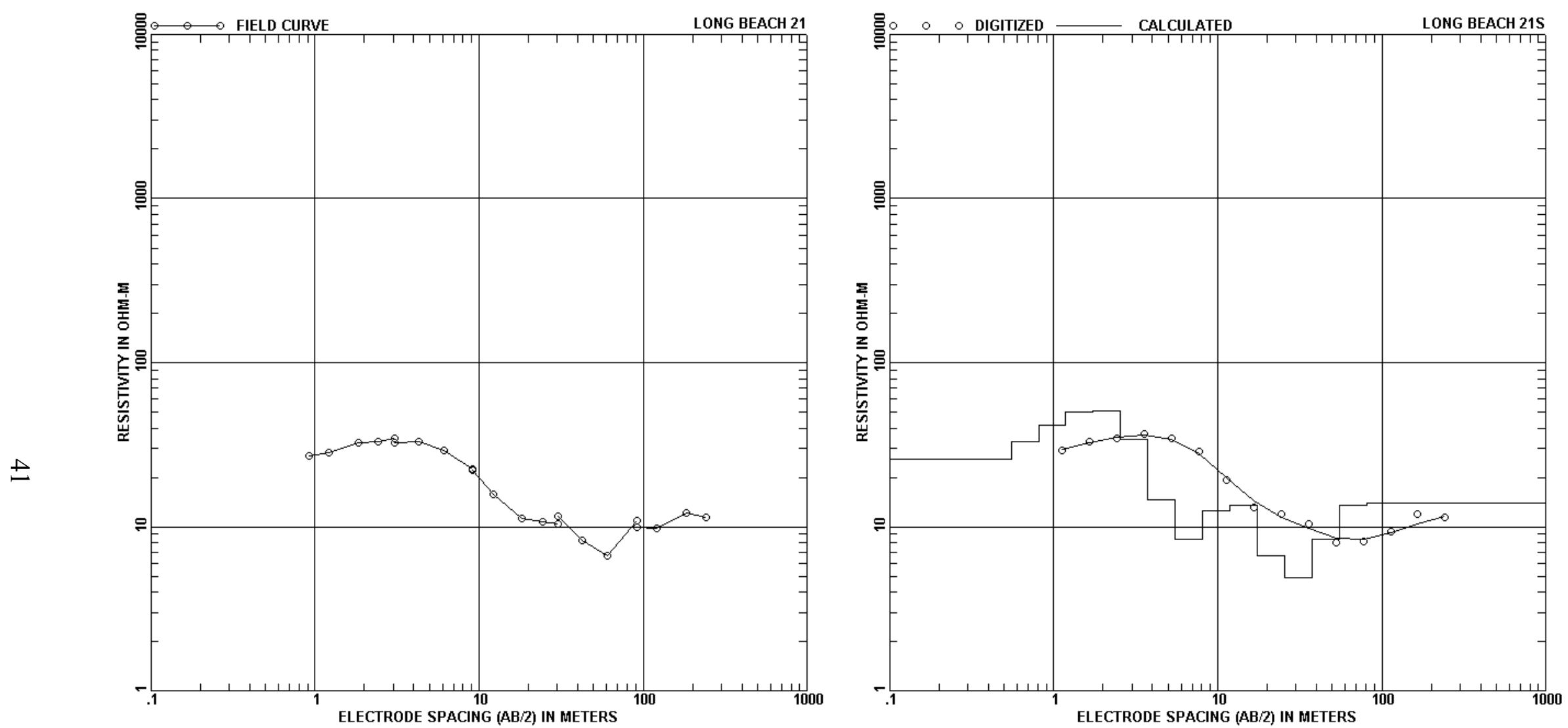

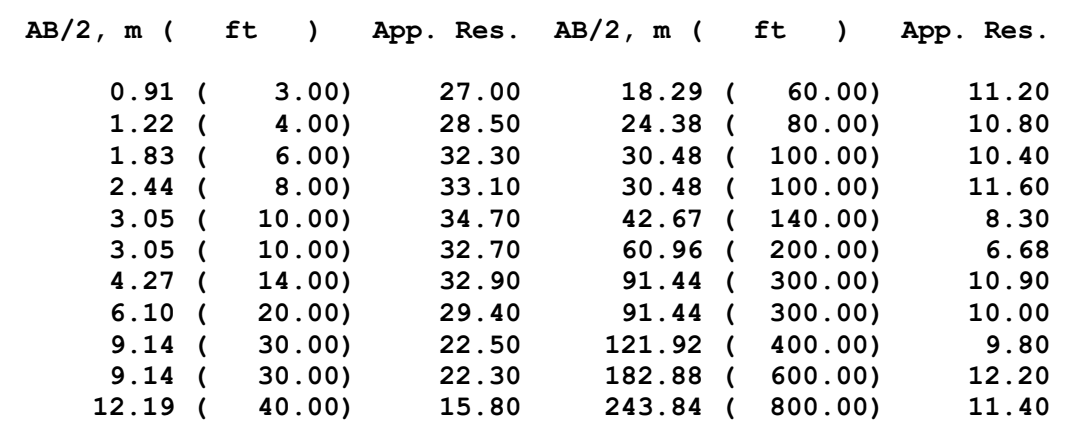

DEPTH, $m$ ( ft ) RESIS. DEPTH, m ( ft

$\begin{array}{rlr}0.55 & ( & 1.80) \\ 0.81 & ( & 2.65) \\ 1.19 & ( & 3.89) \\ 1.74 & ( & 5.71) \\ 2.55 & ( & 8.38) \\ 3.75 & ( & 12.29) \\ 5.50 & ( & 18.05)\end{array}$

25.88

33.15

41.29

50.16

51.15

14.54

$\begin{array}{rlr}8.07 & ( & 26.49) \\ 11.85 & ( & 38.88) \\ 17.39 & ( & 57.07) \\ 25.53 & ( & 83.76) \\ 37.47 & ( & 122.95) \\ 55.01 & ( & 180.47) \\ 80.74 & (264.89) \\ 99999.00 & (99999.00)\end{array}$

RESIS.

8.42

12.43

13.54

6.67

4.93

8.35

13.43

13.94 

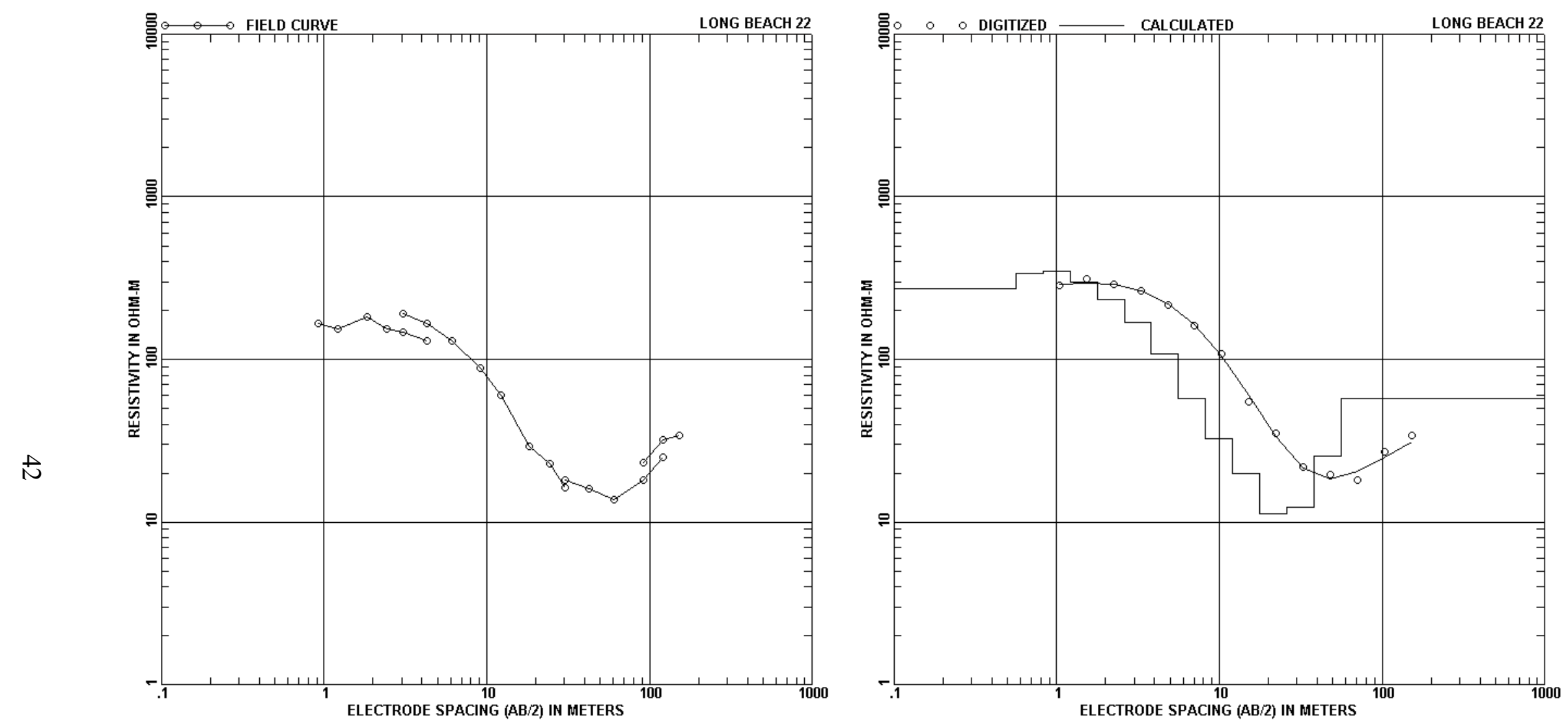

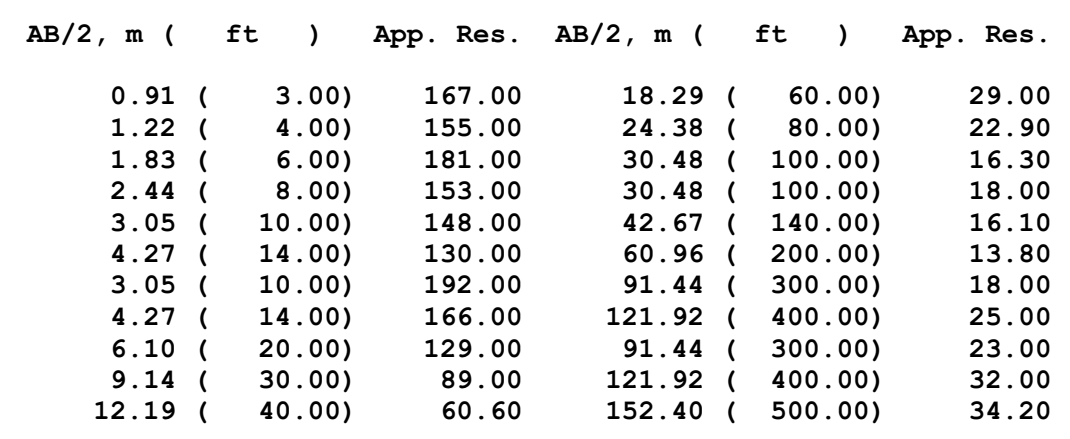

DEPTH, $m$ ( ft )

\begin{tabular}{|c|c|c|c|c|c|}
\hline 0.56 & $1.84)$ & 274.18 & 8.23 & $27.00)$ & 57.06 \\
\hline 0.82 & $2.70)$ & 339.08 & 12.08 & $39.63)$ & 32.56 \\
\hline 1.21 & $3.96)$ & 348.08 & 17.73 & $58.17)$ & 19.77 \\
\hline 1.77 & $5.82)$ & 299.75 & 26.02 & $85.38)$ & 11.32 \\
\hline 2.60 & $8.54)$ & 233.32 & 38.20 & $125.32)$ & 12.35 \\
\hline 3.82 & $12.53)$ & 169.99 & 56.07 & $183.95)$ & 25.36 \\
\hline 5.61 & $18.39)$ & 107.74 & 99999.00 & $(99999.00)$ & 57.32 \\
\hline
\end{tabular}



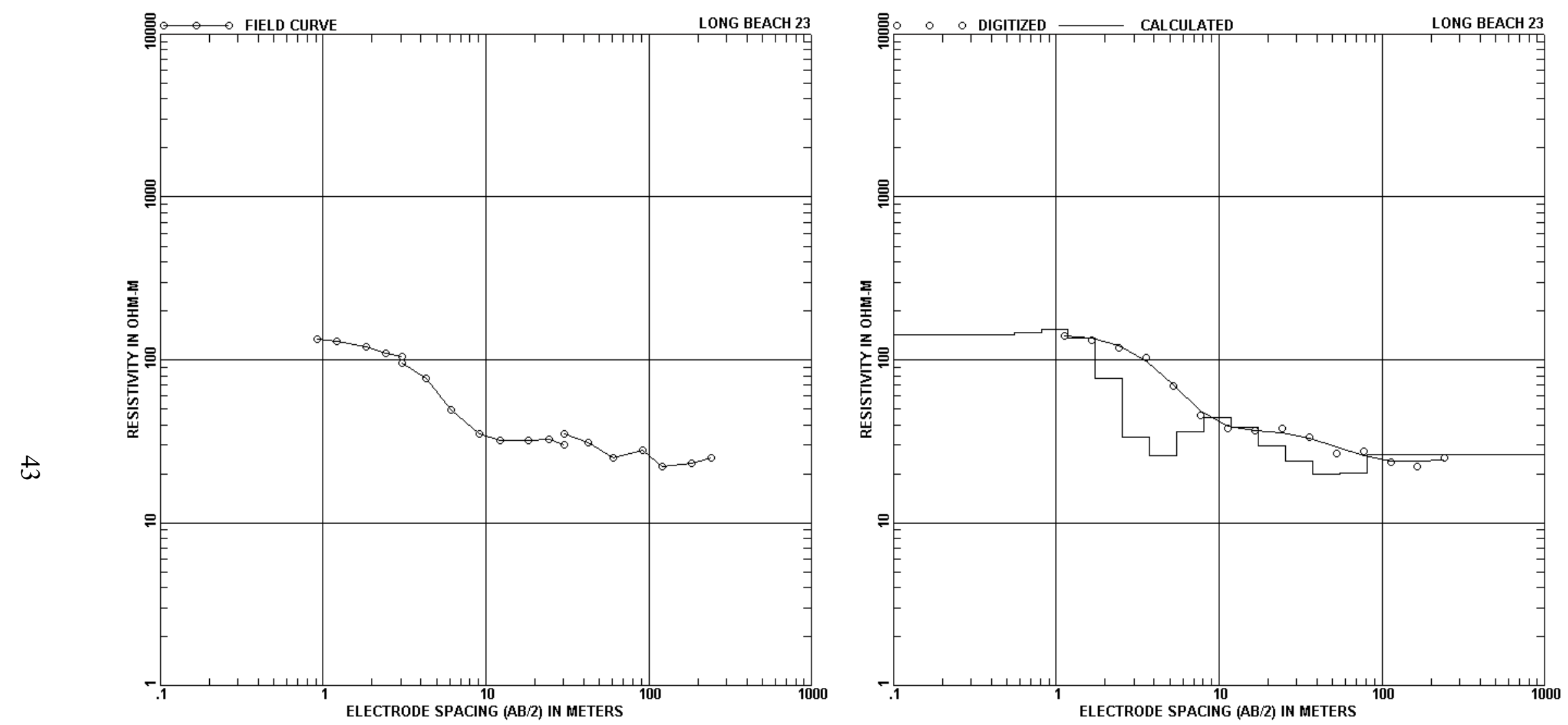

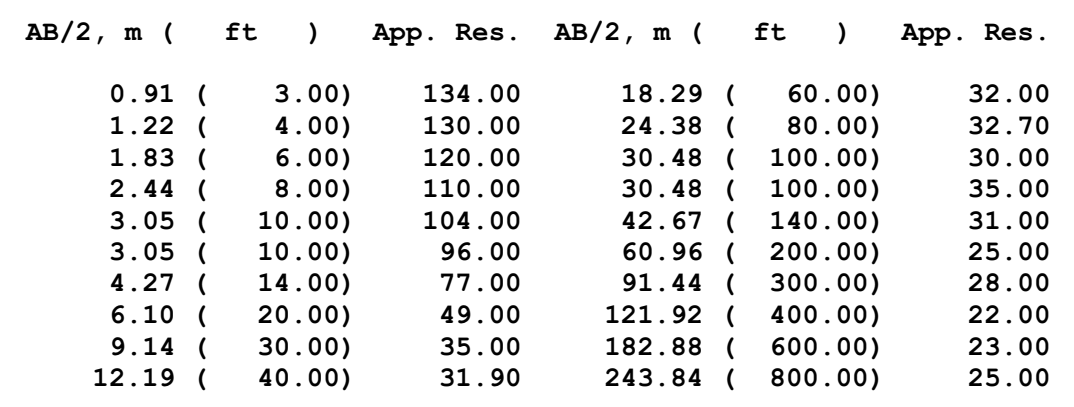

\begin{tabular}{|c|c|c|c|c|c|}
\hline DEPTH， m ( & ft, & RESIS. & DEPTH， m ( & ft & RESIS. \\
\hline 0.551 & $1.80)$ & 142.35 & 8.07 & $26.49)$ & 36.17 \\
\hline $0.81 i$ & $2.65)$ & 146.68 & 11.85 & $38.88)$ & 44.14 \\
\hline $1.19 i$ & $3.89)$ & 153.90 & 17.39 & $57.07)$ & 38.39 \\
\hline $1.74 \%$ & $5.71)$ & 135.40 & 25.53 & $83.76)$ & 29.49 \\
\hline $2.55 i$ & $8.38)$ & 76.96 & 37.47 & $122.95)$ & 23.86 \\
\hline $3.75 i$ & $12.29)$ & 33.63 & 55.01 & $(180.47)$ & 19.97 \\
\hline \multirow[t]{2}{*}{$5.50 i$} & $18.05)$ & 25.90 & 80.74 & ( 264.89) & 20.18 \\
\hline & & & 99999.00 & $(99999.00)$ & 26.12 \\
\hline
\end{tabular}



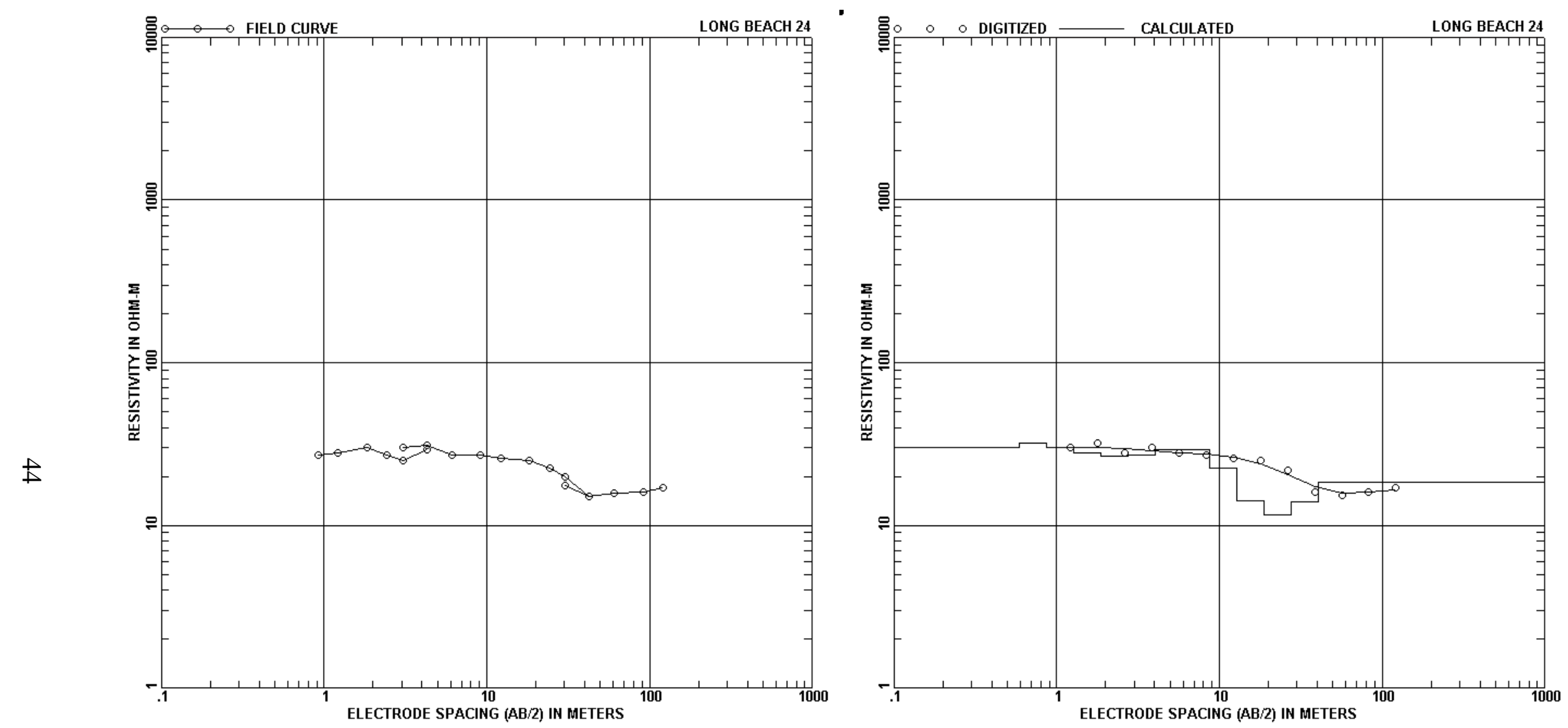

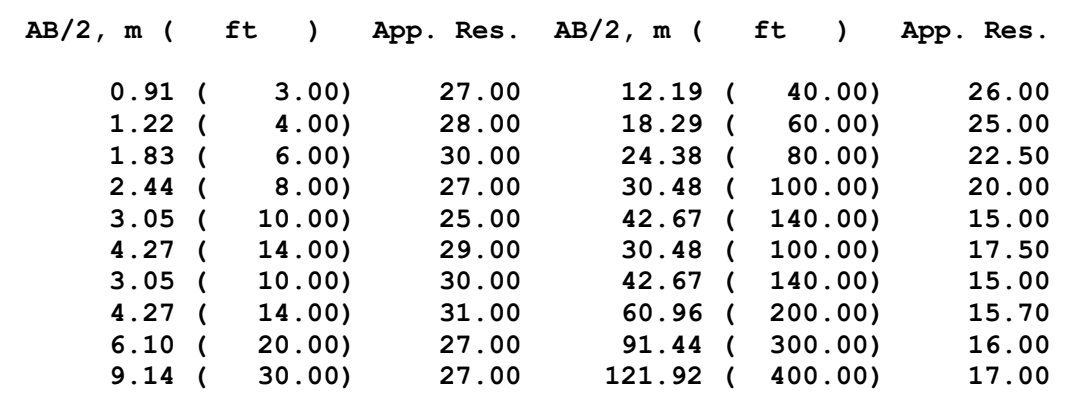

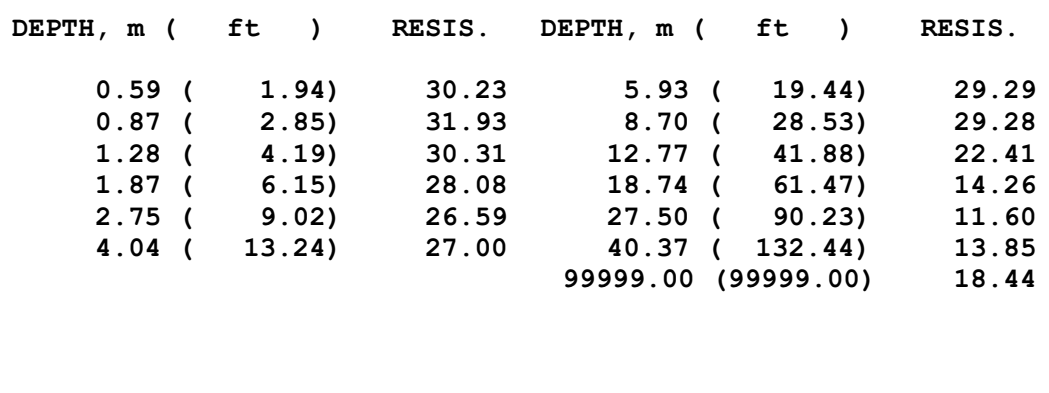



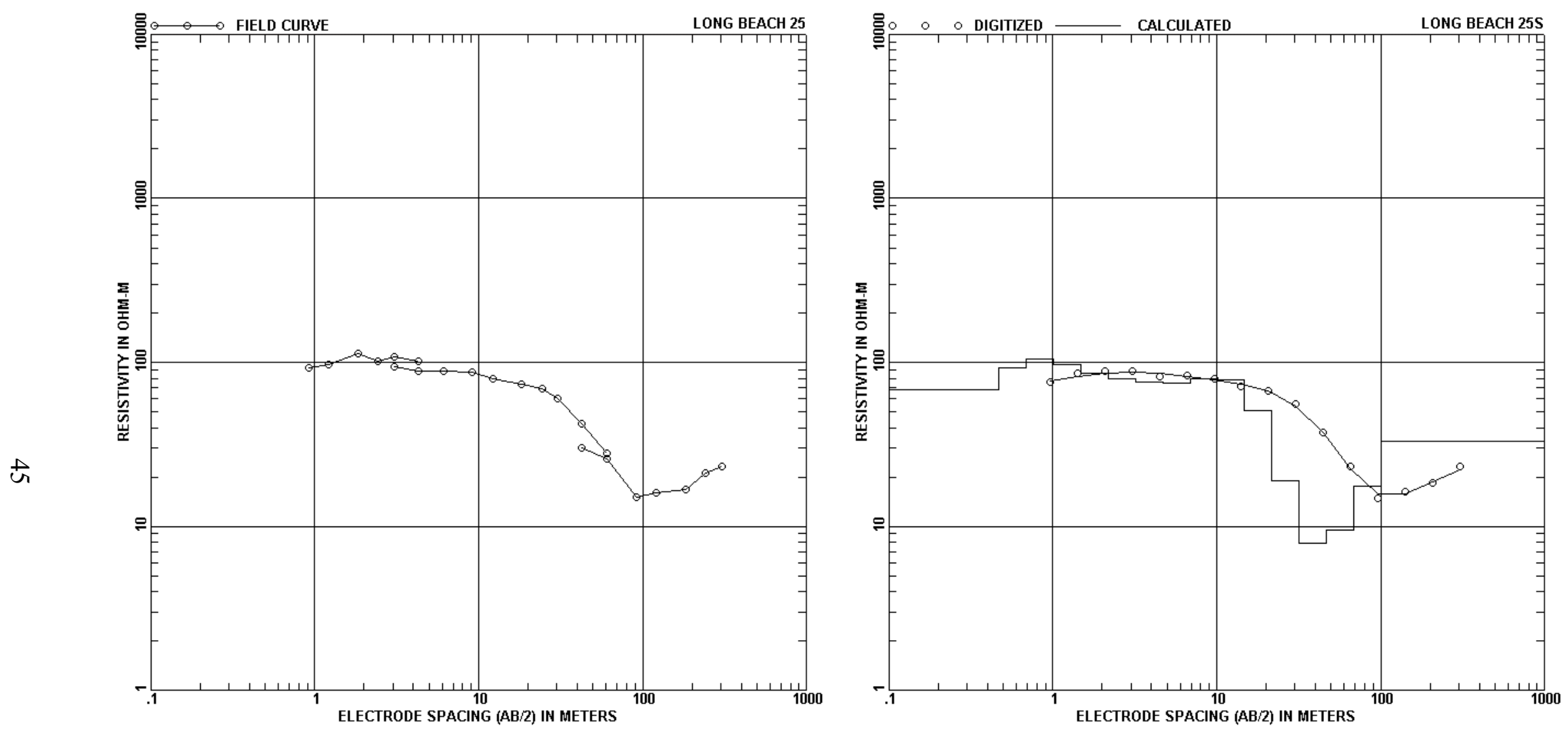

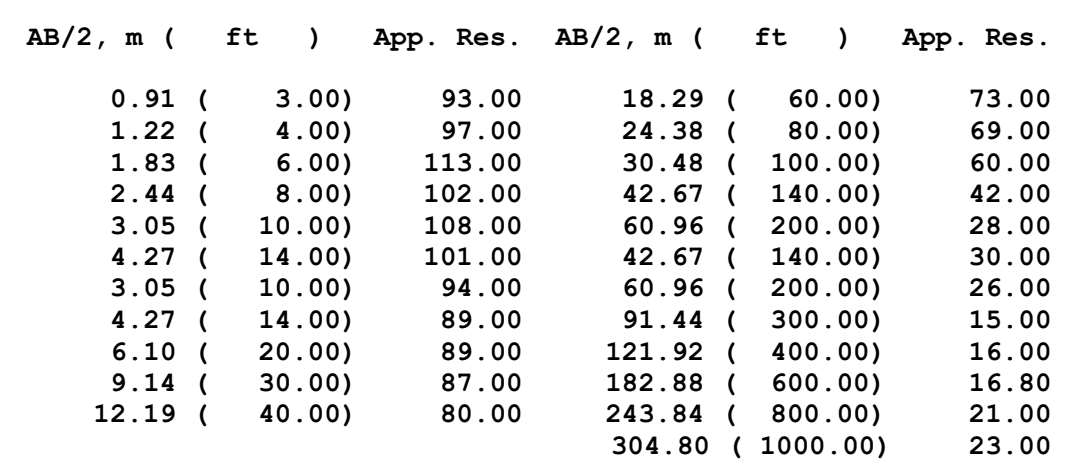

DEPTH， m ( ft )

\begin{tabular}{|c|c|}
\hline .47 & \\
\hline 0.6 & \\
\hline 1.01 & $3.31)$ \\
\hline 1.48 & $4.86)$ \\
\hline 2.17 & $7.13)$ \\
\hline 3.19 & $(0.47)$ \\
\hline .68 & 15.37) \\
\hline 5.88 & $22.56)$ \\
\hline
\end{tabular}
RESIS.

68.38

92.89 104.67

97.39

85.49

85.49

79.37

75.54

74.53
DEPTH, m ( ft )

RESIS.

$10.09(33.11)$

$14.81(48.60)$

79.81

$31.91(104.71) \quad 18.84$

$\begin{array}{lll}46.84 & (153.69) & 7.88\end{array}$

$68.76(225.58) \quad 9.51$

100.92 ( 331.11$) \quad 17.69$ $\begin{array}{lll}99999.00 & (99999.00) & 33.25\end{array}$ 

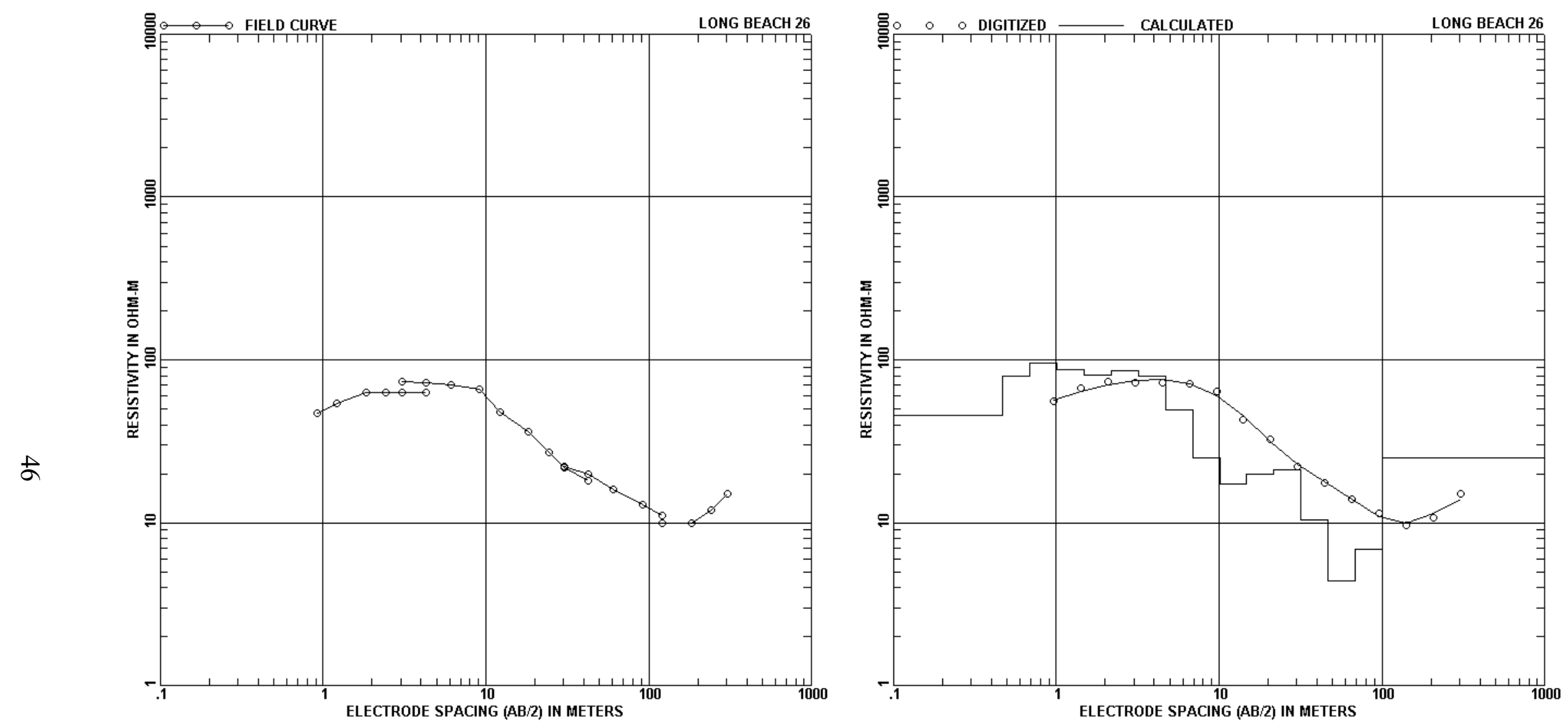

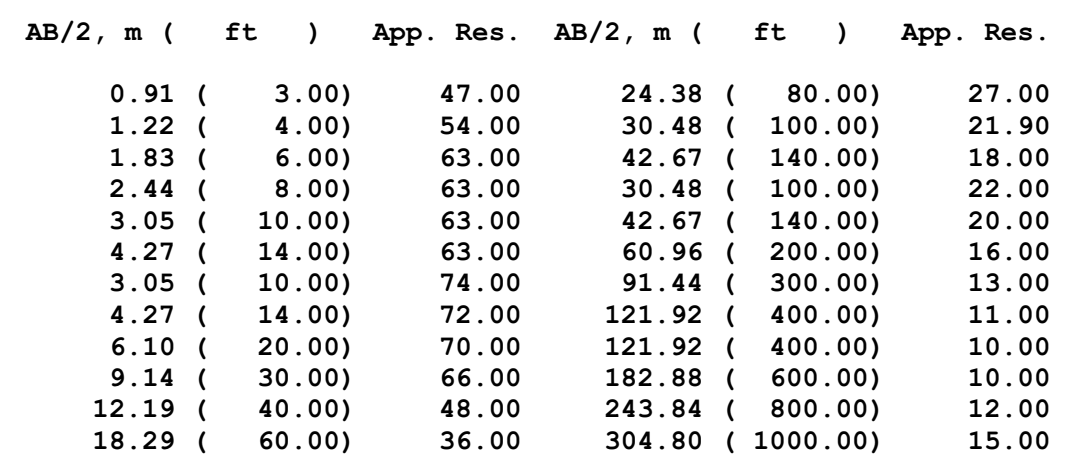

DEPTH, $m$ ( ft ) RESIS, DEPTH, m ( ft

\begin{tabular}{|c|c|}
\hline .47 & \\
\hline 0. & 2 \\
\hline 1.01 & 3. \\
\hline 1.48 & 4. \\
\hline 2.17 & 7. \\
\hline 3.19 & 10. \\
\hline 4. & 15 \\
\hline 6.88 & 22.5 \\
\hline
\end{tabular}

45.39

79.57

95.44

86.62

80.41

80.41

85.82

49.66
$10.09(33.11)$

$14.81(48.60)$

21.74 ( 71.34)

31.91 ( 104.71)

46.84 ( 153.69$)$

$68.76(225.58)$

100.92 ( 331.11$)$

$9999.00(99999.00)$
RESIS.

25.16

17.37

19.86

21.27

10.46

4.39

6.92 

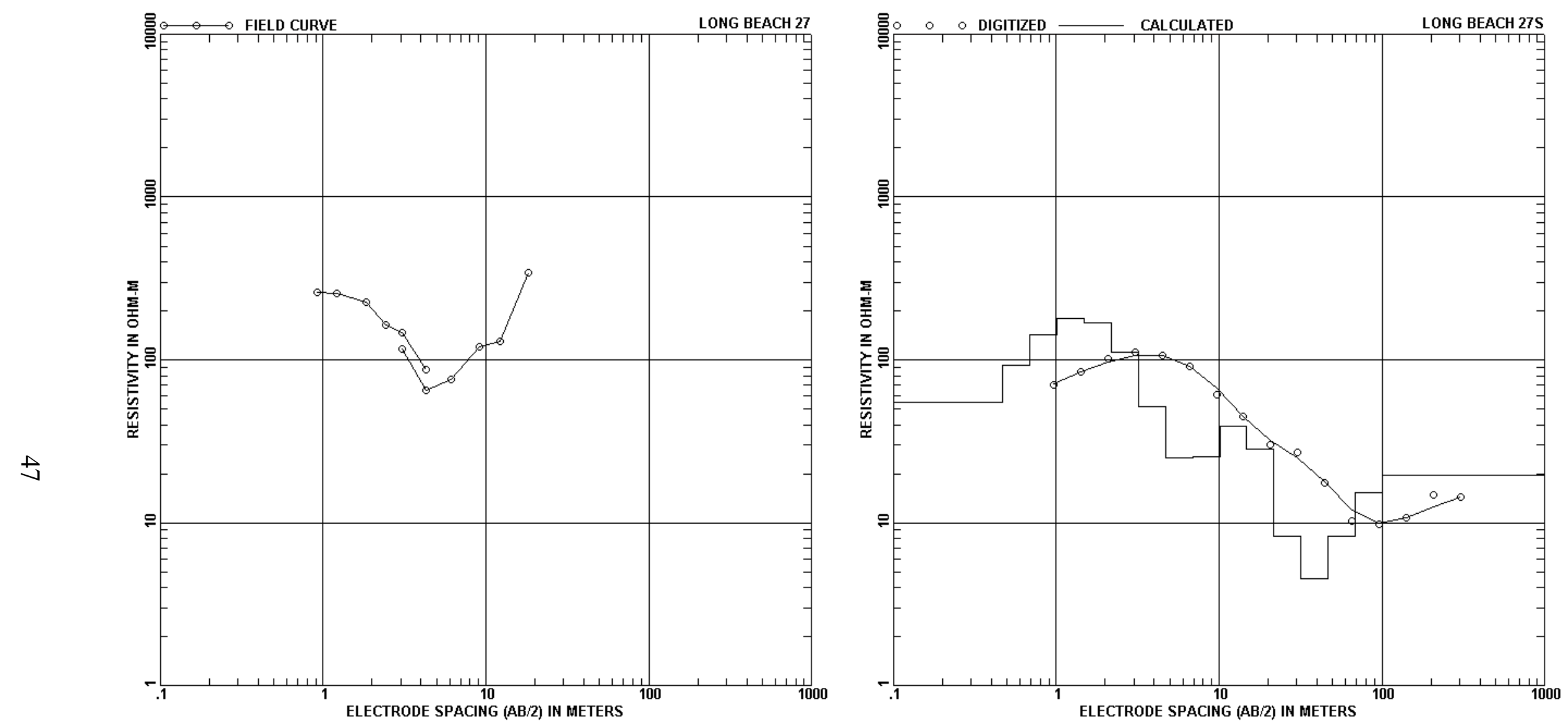

$\begin{array}{rrrrrr}A B / 2, \mathrm{~m} \text { ( } & \text { ft ) } & \text { App. Res. } & A B / 2, m(\text { ft ) } & \text { App. Res. } \\ 0.91( & 3.00) & 261.00 & 3.05( & 10.00) & 116.00 \\ 1.22( & 4.00) & 256.00 & 4.27( & 14.00) & 65.00 \\ 1.83( & 6.00) & 228.00 & 6.10( & 20.00) & 76.00 \\ 2.44( & 8.00) & 165.00 & 9.14( & 30.00) & 121.00 \\ 3.05( & 10.00) & 147.00 & 12.19( & 40.00) & 129.00 \\ 4.27( & 14.00) & 87.00 & 18.29( & 60.00) & 344.00\end{array}$

\begin{tabular}{|c|c|c|c|c|c|}
\hline $\mathrm{PTH}, \mathrm{m}$ ( & $f t$ & RESIS. & DEPTH， $\mathrm{m}$ & ft & RESIS. \\
\hline 0.47 ( & $1.54)$ & 55.15 & 10.09 & $33.11)$ & 25.60 \\
\hline 0.69 ( & $2.26)$ & 92.24 & 14.81 & $48.60)$ & 39.32 \\
\hline 1.01 ( & $3.31)$ & 142.00 & 21.74 & $71.34)$ & 28.20 \\
\hline $1.48 \%$ & $4.86)$ & 178.61 & 31.91 & $104.71)$ & 8.21 \\
\hline 2.17 ( & $7.13)$ & 169.05 & 46.84 & $153.69)$ & 4.54 \\
\hline $3.19 i$ & $10.47)$ & 111.21 & 68.76 & ( 225.58) & 8.31 \\
\hline $4.68 i$ & $15.37)$ & 51.75 & 100.92 & ( 331.11$)$ & 15.27 \\
\hline $6.88 i$ & $22.56)$ & 25.11 & 99999.00 & (99999.00) & 19.47 \\
\hline
\end{tabular}



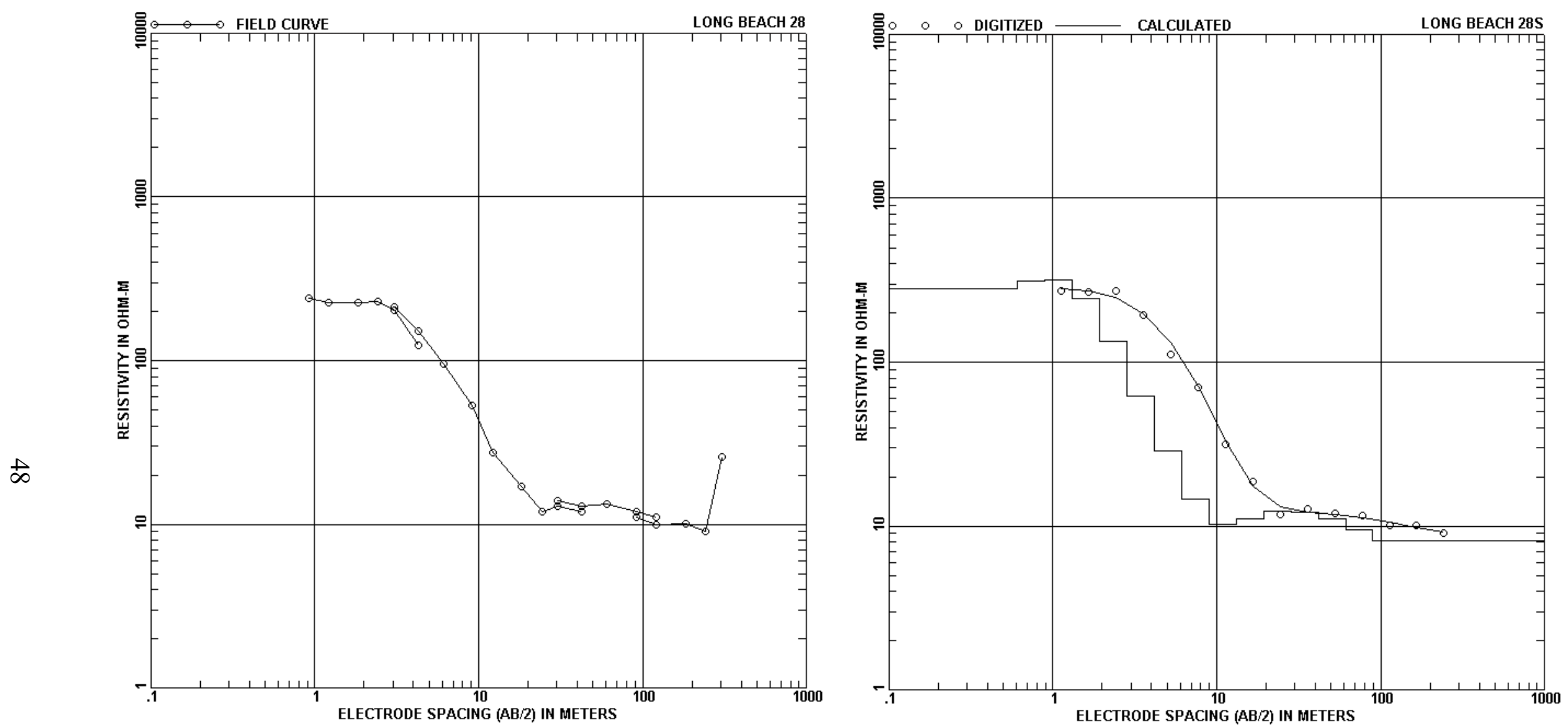

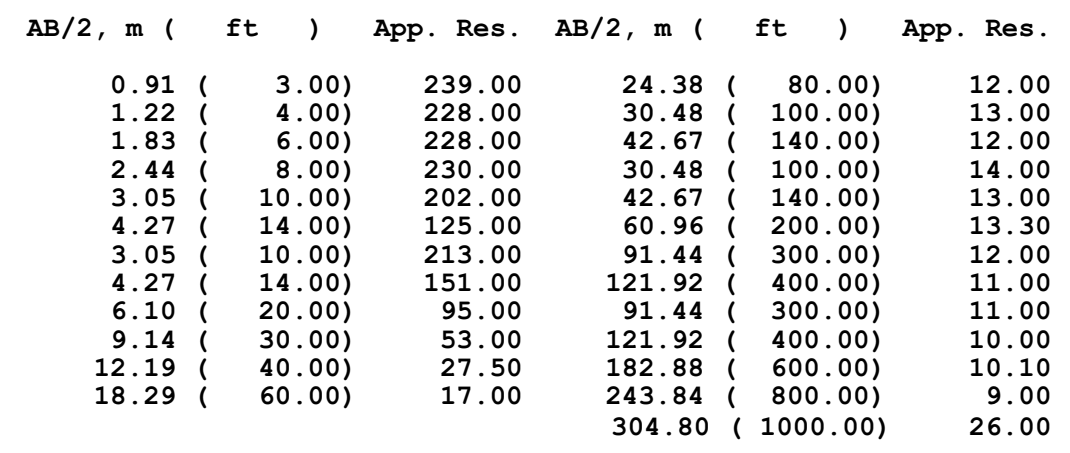

\begin{tabular}{|c|c|c|c|c|c|}
\hline DEPTH， m ( & ft , & RESIS. & DEPTH， m & $f t$ & RESIS. \\
\hline 0.61( & $2.01)$ & 241.73 & 8.97 & $29.43)$ & 14.10 \\
\hline $0.90 i$ & $2.94)$ & 267.95 & 13.17 & $43.20)$ & 12.29 \\
\hline 1.32( & $4.32)$ & 276.46 & 19.33 & $63.41)$ & 11.73 \\
\hline $1.93 i$ & $6.34)$ & 227.51 & 28.37 & $93.07)$ & 11.94 \\
\hline $2.84 i$ & $9.31)$ & 138.18 & 41.64 & $136.61)$ & 11.91 \\
\hline $4.16 i$ & $13.66)$ & 63.61 & 61.12 & $200.52)$ & 10.94 \\
\hline 6.111 & $20.05)$ & 25.60 & 89.71 & $(294.32)$ & 9.54 \\
\hline & & & 99999.00 & $(99999.00)$ & 8.16 \\
\hline
\end{tabular}



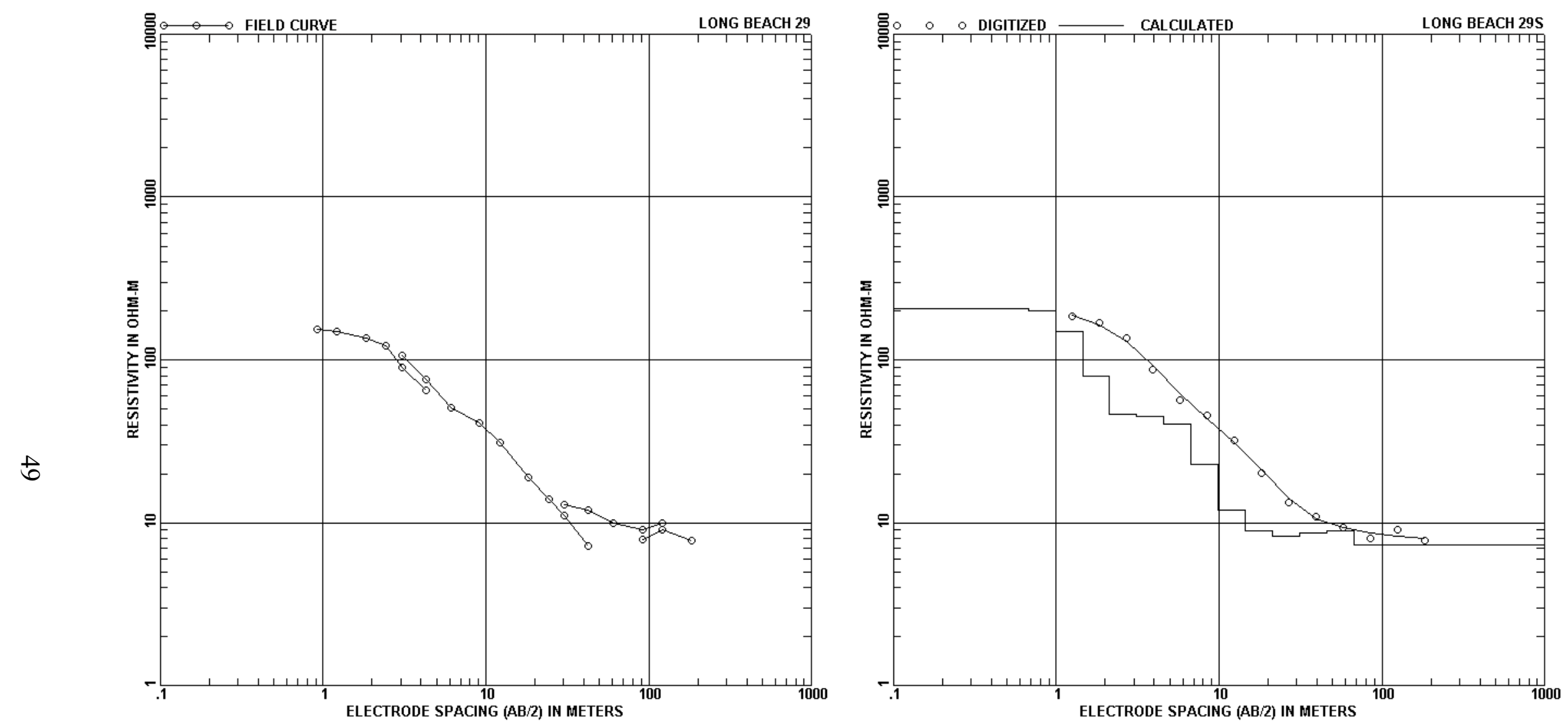

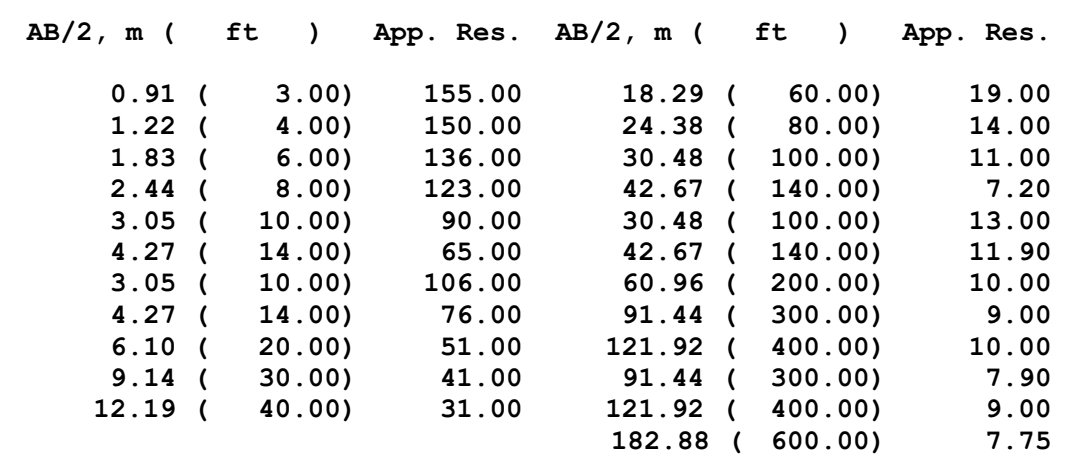

\begin{tabular}{|c|c|c|c|c|c|}
\hline DEPTH， m ( & ft ) & RESIS. & DEPTH， m ( & ft & RESIS. \\
\hline 0.671 & $2.21)$ & 206.65 & 9.88 & $32.40)$ & 22.74 \\
\hline $0.99 i$ & $3.24)$ & 201.17 & 14.50 & $47.56)$ & 11.87 \\
\hline $1.45 i$ & $4.76)$ & 148.49 & 21.28 & $69.80)$ & 8.93 \\
\hline $2.13 i$ & $6.98)$ & 79.14 & 31.23 & $(102.46)$ & 8.24 \\
\hline $3.12 i$ & $10.25)$ & 46.66 & 45.84 & ( 150.39$)$ & 8.63 \\
\hline $4.58 i$ & $15.04)$ & 45.09 & 67.28 & $(220.74)$ & 8.95 \\
\hline $6.73 i$ & $22.07)$ & 40.61 & 99999.00 & $(99999.00)$ & 7.32 \\
\hline
\end{tabular}



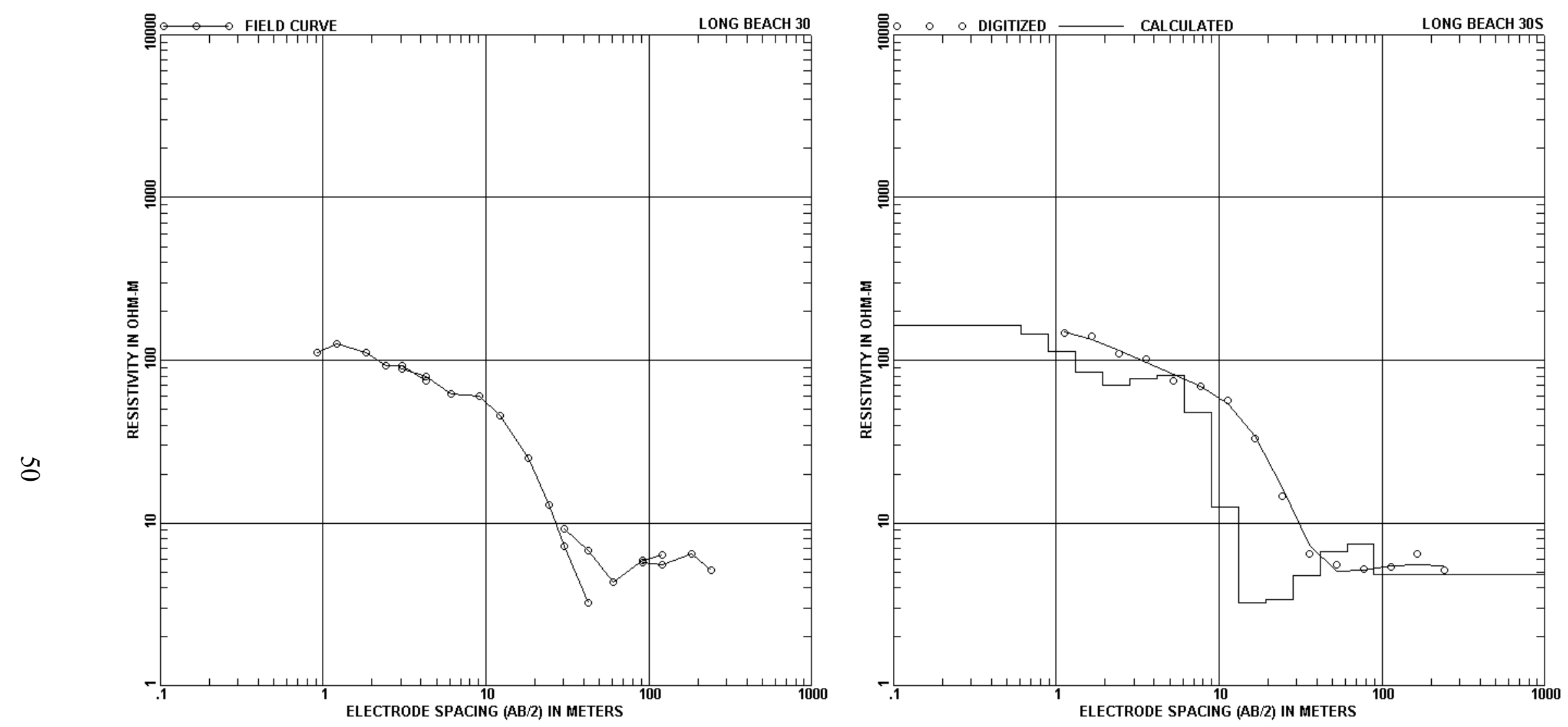

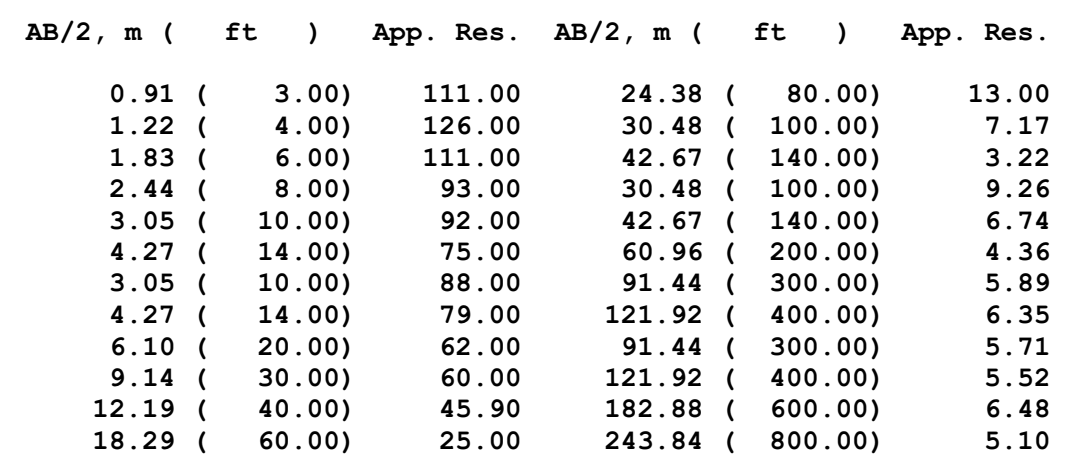

\begin{tabular}{|c|c|c|c|c|c|}
\hline DEPTH， m ( & ft & RESIS. & DEPTH， m ( & ft & RESIS. \\
\hline 0.611 & $2.01)$ & 163.29 & 8.97 & $29.43)$ & 47.73 \\
\hline $0.90 i$ & $2.94)$ & 144.19 & 13.17 & $43.20)$ & 12.61 \\
\hline $1.32 \%$ & 4.32) & 112.76 & 19.33 & $63.41)$ & 3.23 \\
\hline $1.93 i$ & $6.34)$ & 84.72 & 28.37 & $93.07)$ & 3.36 \\
\hline $2.84 i$ & $9.31)$ & 70.48 & 41.64 & $136.61)$ & 4.76 \\
\hline 4.16 & $13.66)$ & 76.84 & 61.12 & $(200.52)$ & 6.63 \\
\hline $6.11 i$ & $20.05)$ & 80.93 & 89.71 & $(294.32)$ & 7.42 \\
\hline & & & 99999.00 & $(99999.00)$ & 4.83 \\
\hline
\end{tabular}



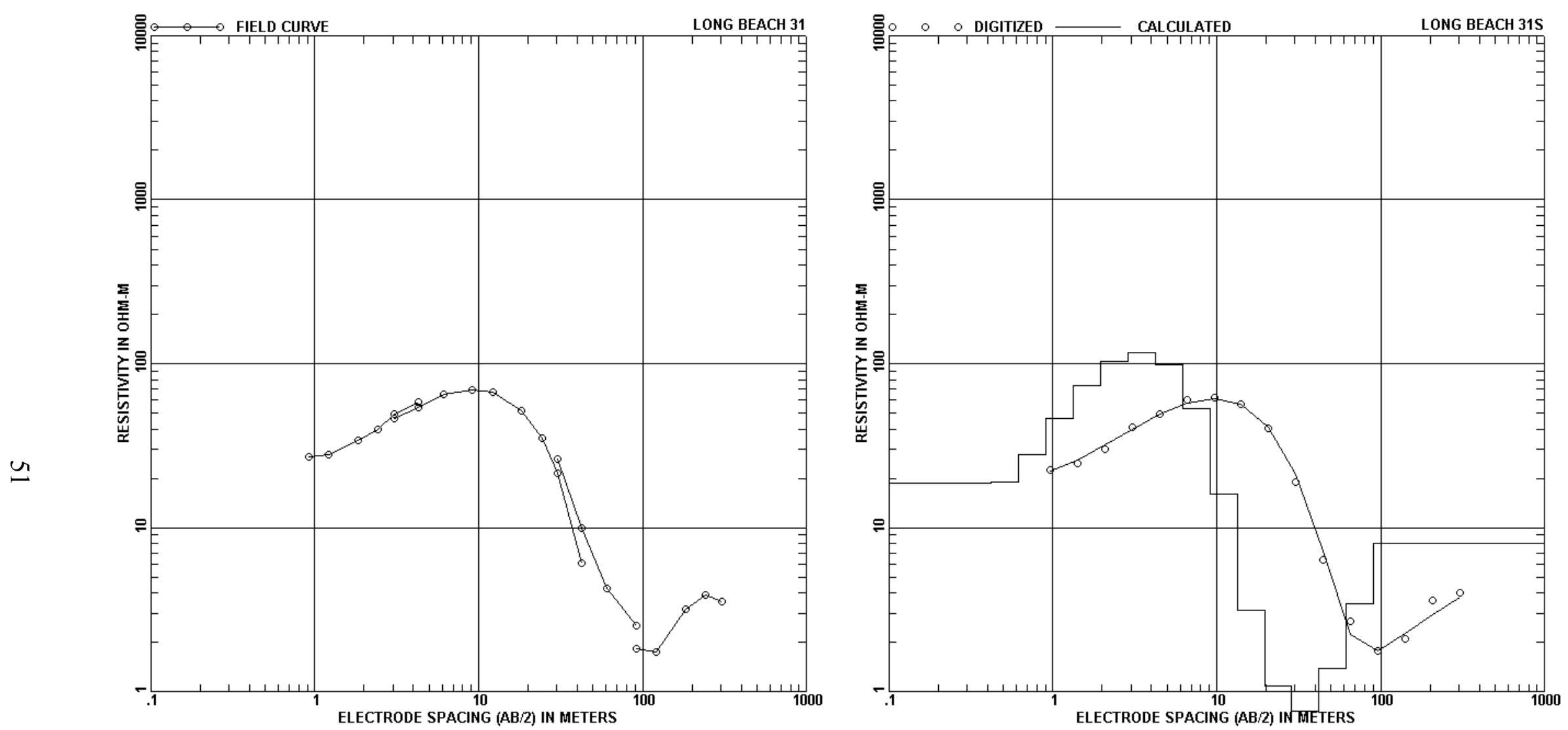

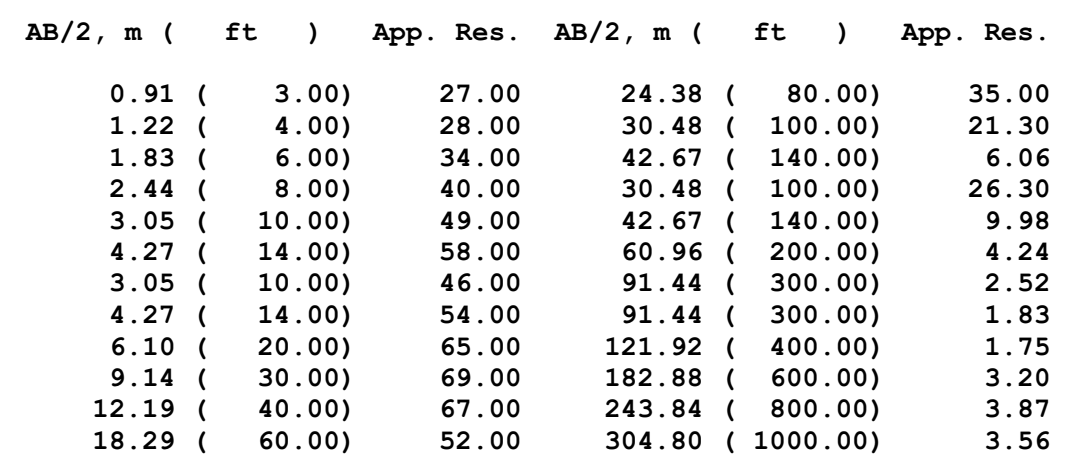

DEPTH, $m$ ( ft ) RESIS, DEPTH, m ( ft )

\begin{tabular}{|c|c|c|c|c|c|}
\hline 0.42 & $1.38)$ & 18.70 & 9.08 & $29.80)$ & 53.29 \\
\hline 0.62 & $2.03)$ & 19.04 & 13.33 & $43.74)$ & 15.98 \\
\hline 0.91 & $2.98)$ & 28.01 & 19.57 & $64.20)$ & 3.14 \\
\hline 1.33 & $4.37)$ & 46.68 & 28.72 & $94.23)$ & 1.08 \\
\hline 1.96 & $6.42)$ & 73.92 & 42.16 & $138.32)$ & 0.76 \\
\hline 2.87 & $9.42)$ & 102.90 & 61.88 & $203.02)$ & 1.38 \\
\hline 4.22 & 13.83) & 117.50 & 90.83 & $298.00)$ & 3.45 \\
\hline 6.19 & $20.30)$ & 99.16 & 99999.00 & $(99999.00)$ & 8.02 \\
\hline
\end{tabular}



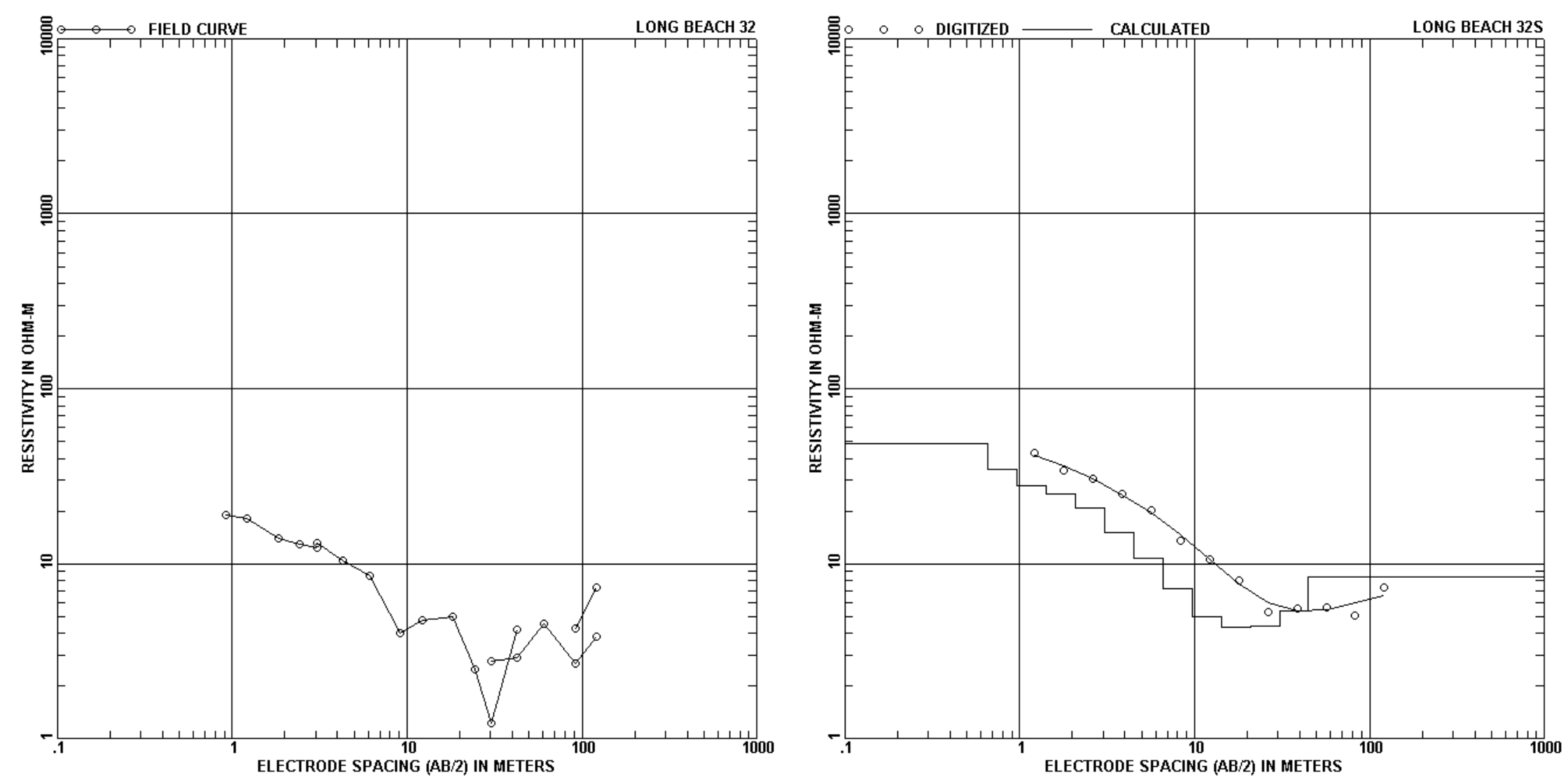

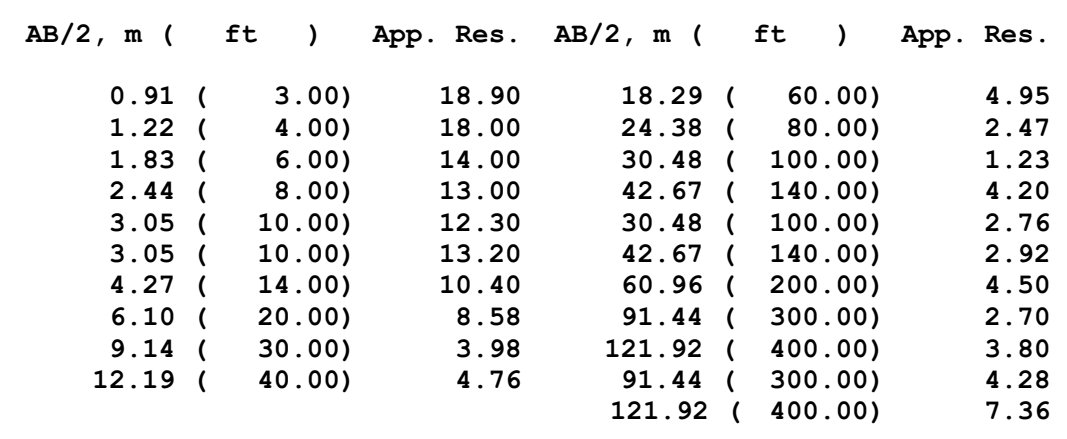

\begin{tabular}{|c|c|c|c|c|c|}
\hline DEPTH， m ( & ft & RESIS. & DEPTH， m ( & ft & RESIS. \\
\hline 0.661 & $2.16)$ & 48.67 & 6.58 & $21.60)$ & 10.67 \\
\hline $0.97 i$ & $3.17)$ & 34.45 & 9.66 & $31.70)$ & 7.23 \\
\hline $1.42 \%$ & $4.65)$ & 27.78 & 14.18 & $46.54)$ & 4.94 \\
\hline $2.08 i$ & $6.83)$ & 25.08 & 20.82 & $68.31)$ & 4.31 \\
\hline $3.06 i$ & $10.03)$ & 20.91 & 30.56 & $100.26)$ & 4.38 \\
\hline 4.49 i & $14.72)$ & 15.13 & 44.85 & $147.16)$ & 5.34 \\
\hline & & & 99999.00 & $(99999.00)$ & 8.41 \\
\hline
\end{tabular}



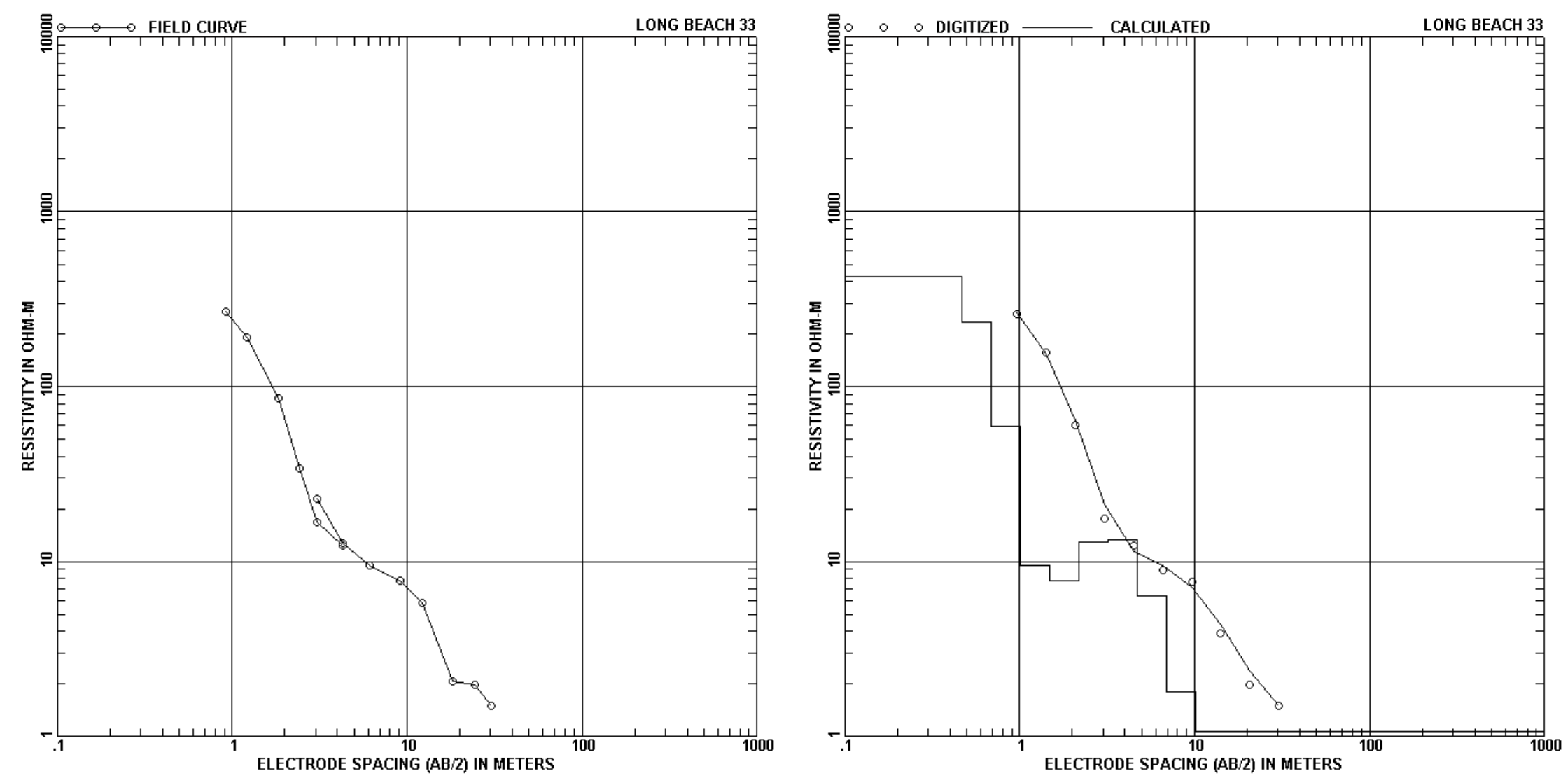

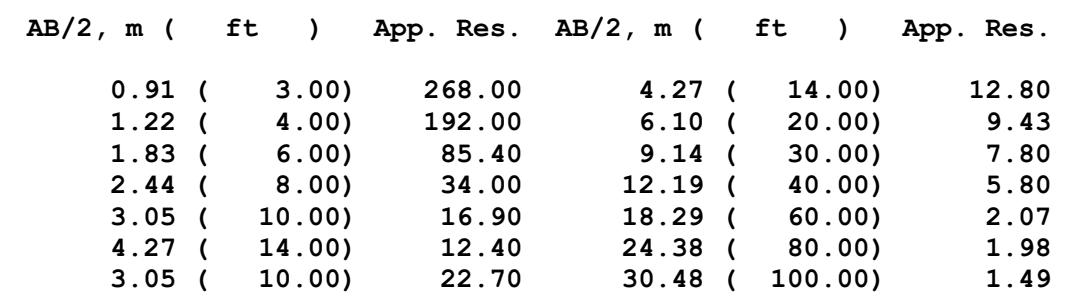

\begin{tabular}{|c|c|c|c|c|c|}
\hline DEPTH， m ( & ) & RESIS. & DEPTH， m & $\mathrm{ft}$ & RESIS. \\
\hline 0.47 & $1.54)$ & 424.11 & 3.19 & $10.47)$ & 12.87 \\
\hline $0.69 i$ & $2.26)$ & 234.24 & 4.68 & $15.37)$ & 13.25 \\
\hline 1.01 ( & $3.31)$ & 59.39 & 6.88 & $22.56)$ & 6.32 \\
\hline 1.48 ( & $4.86)$ & 9.50 & 10.09 & $33.11)$ & 1.79 \\
\hline 2.17 ( & $7.13)$ & 7.81 & 99999.00 & (99999.00) & 1.06 \\
\hline
\end{tabular}



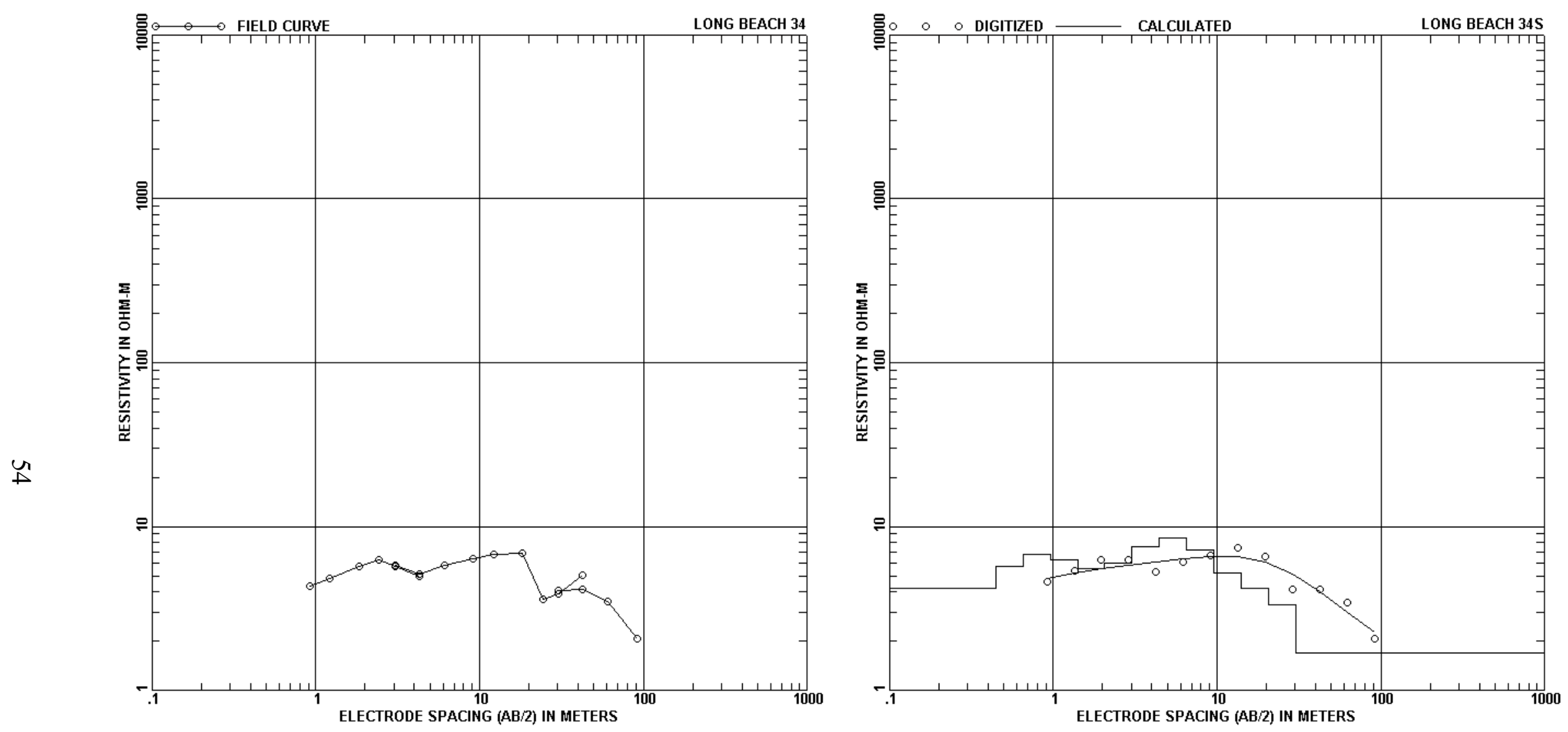

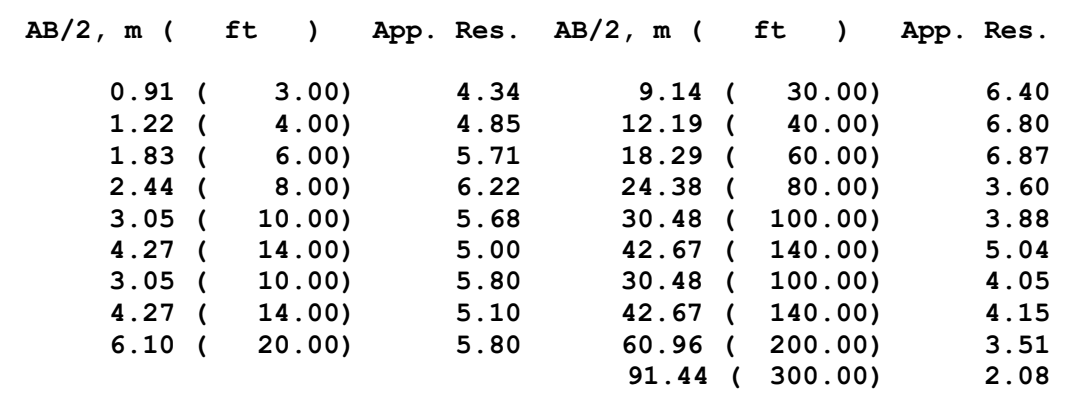

\begin{tabular}{|c|c|c|c|c|c|}
\hline DEPTH， m ( & ft ) & RESIS. & DEPTH， m ( & ft & RESIS. \\
\hline 0.44 & $1.46)$ & 4.19 & 4.44 & $14.58)$ & 7.58 \\
\hline 0.65 & $2.14)$ & 5.75 & 6.52 & $21.40)$ & 8.57 \\
\hline 0.96 & $3.14)$ & 6.75 & 9.57 & $31.41)$ & 7.21 \\
\hline 1.41 & $4.61)$ & 6.29 & 14.05 & $46.11)$ & 5.18 \\
\hline 2.06 & $6.77)$ & 5.55 & 20.63 & $67.67)$ & 4.21 \\
\hline \multirow[t]{2}{*}{3.031} & $9.93)$ & 5.98 & 30.28 & $99.33)$ & 3.33 \\
\hline & & & 99999.00 & $(99999.00)$ & 1.69 \\
\hline
\end{tabular}



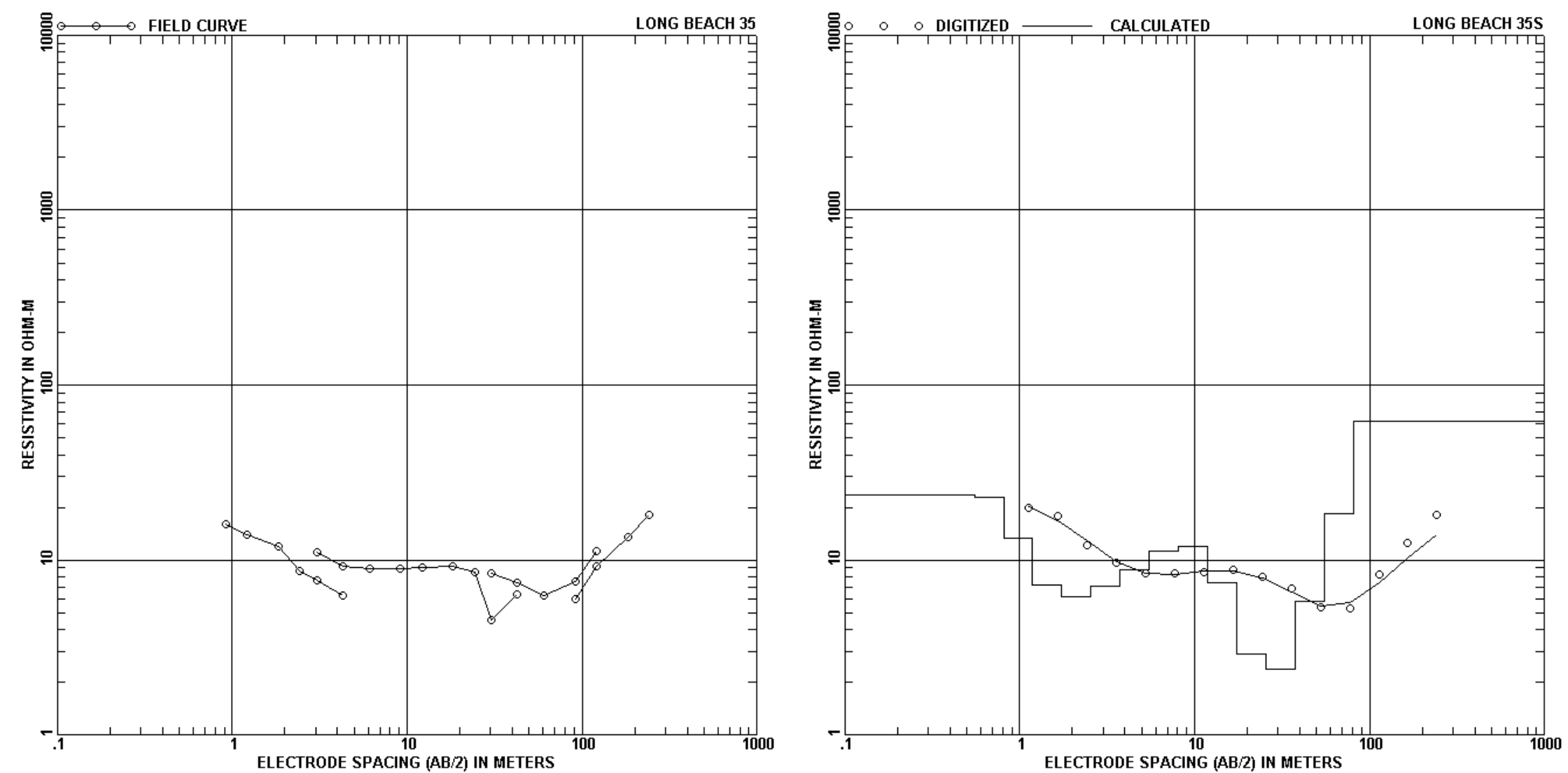

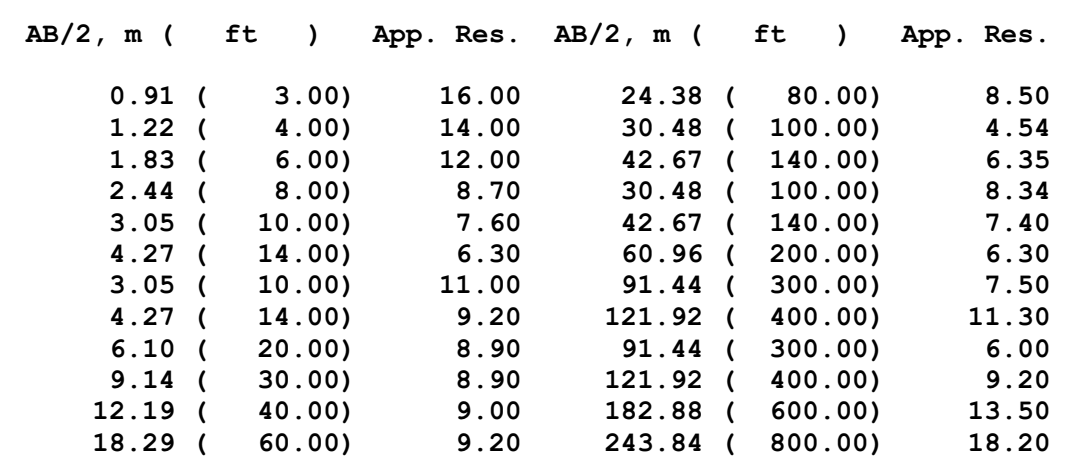

\begin{tabular}{|c|c|c|c|c|c|}
\hline DEPTH， m ( & ft & RESIS. & DEPTH， m ( & ft & RESIS. \\
\hline 0.55 & $1.80)$ & 23.42 & 8.07 & $26.49)$ & 11.29 \\
\hline 0.81 & $2.65)$ & 22.66 & 11.85 & $38.88)$ & 11.98 \\
\hline 1.19 & $3.89)$ & 13.36 & 17.39 & $57.07)$ & 7.42 \\
\hline 1.74 & $5.71)$ & 7.21 & 25.53 & $83.76)$ & 2.89 \\
\hline 2.55 & $8.38)$ & 6.14 & 37.47 & 122.95 ) & 2.37 \\
\hline 3.75 & $12.29)$ & 7.06 & 55.01 & $180.47)$ & 5.79 \\
\hline 5.50 & $18.05)$ & 8.79 & 80.74 & $264.89)$ & 18.44 \\
\hline & & & 99999.00 & $(99999.00)$ & 61.71 \\
\hline
\end{tabular}



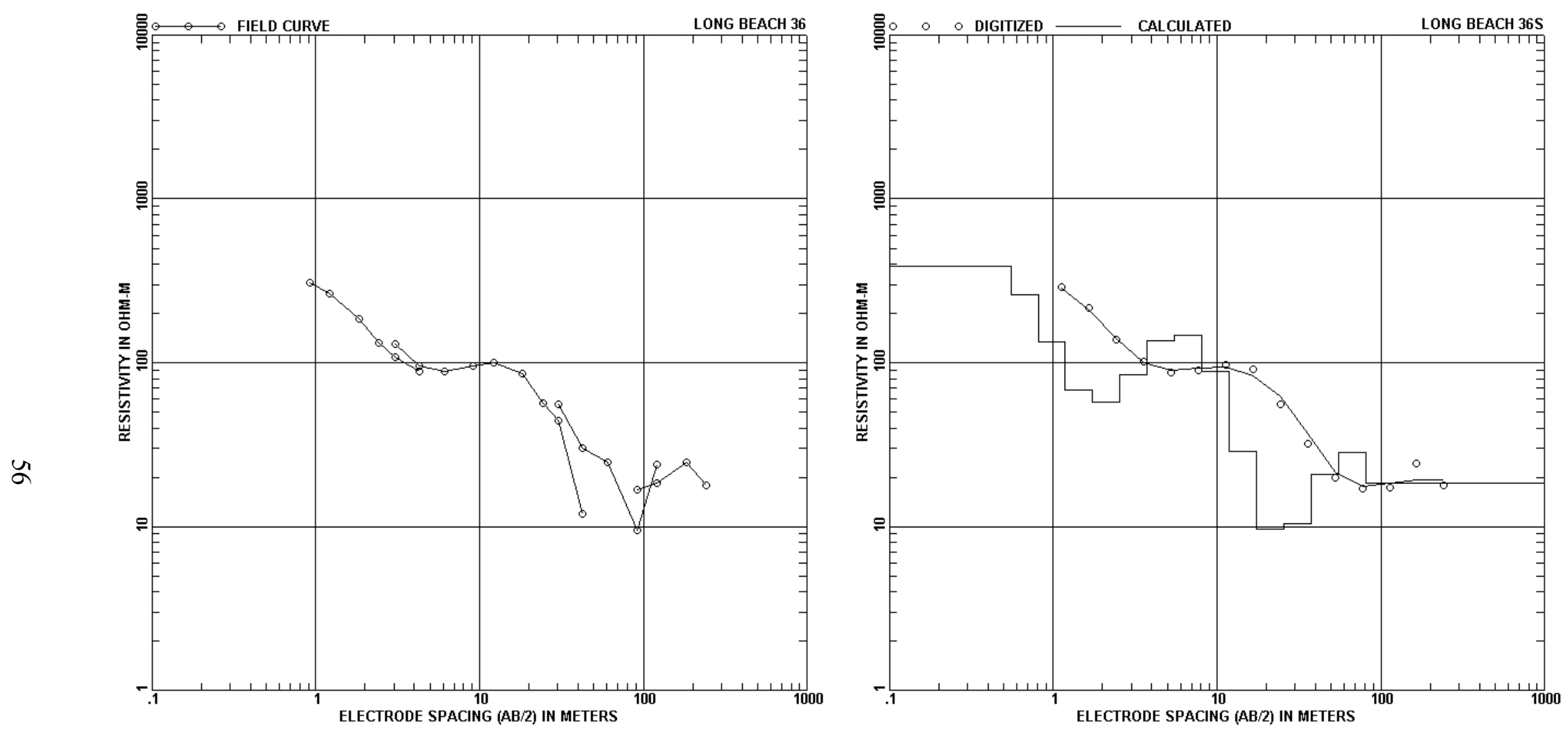

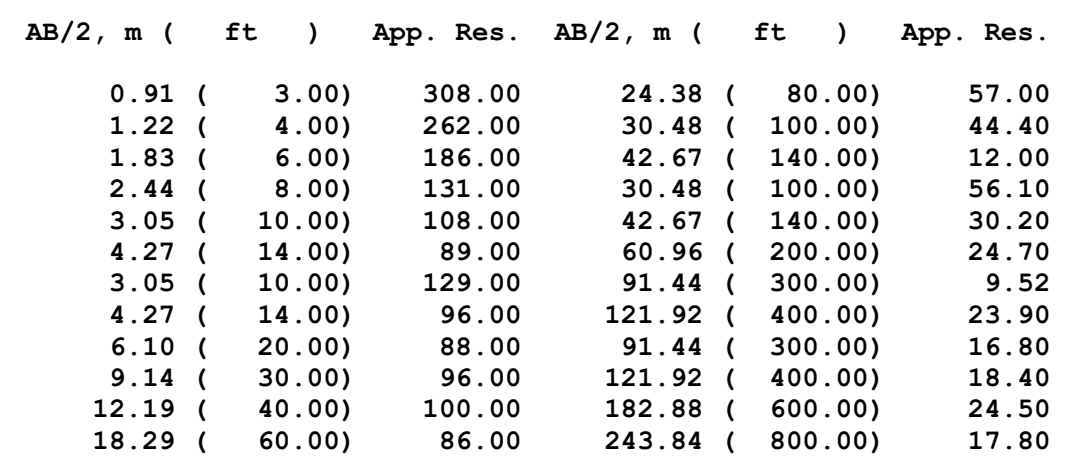

DEPTH， m ( ft )

$\begin{array}{rlr}0.55 & ( & 1.80) \\ 0.81 & ( & 2.65) \\ 1.19 & ( & 3.89) \\ 1.74 & ( & 5.71) \\ 2.55 & ( & 8.38) \\ 3.75 & ( & 12.29) \\ 5.50 & ( & 18.05)\end{array}$

RESIS. DEPTH, $m($ ft

$\begin{array}{rrlr}385.10 & 8.07( & 26.49) & 147.15 \\ 258.13 & 11.85(138.88) & 88.04 \\ 133.95 & 17.39( & 57.07) & 28.79 \\ 68.54 & 25.53( & 83.76) & 9.63 \\ 57.07 & 37.47( & 122.95) & 10.36 \\ 85.07 & 55.01(180.47) & 20.87 \\ 135.47 & 80.74(264.89) & 28.39 \\ & 99999.00 & (99999.00) & 18.35\end{array}$



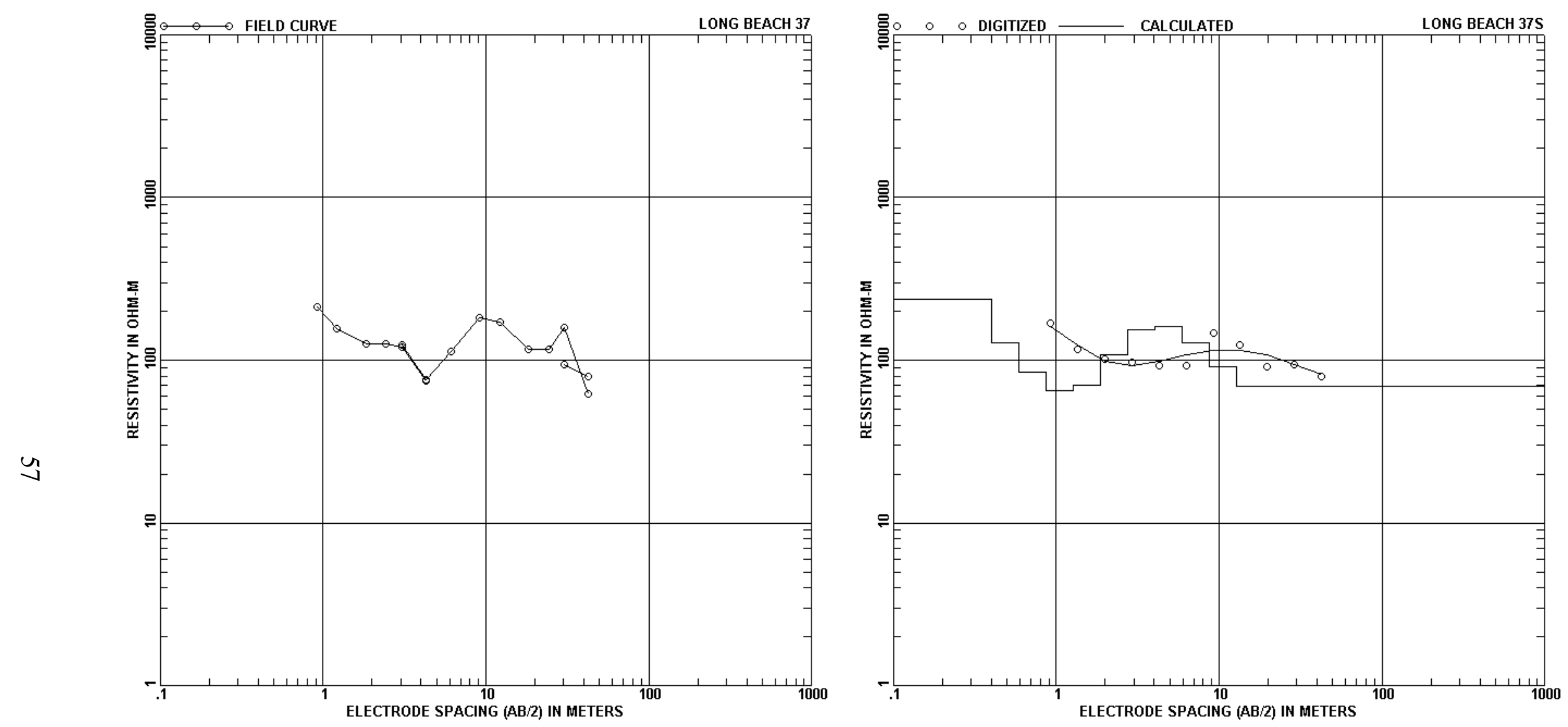

$\begin{array}{rrrrrr}A B / 2, \text { m ( } & \text { ft ) } & \text { App. Res. } & A B / 2, m(\text { ft ) } & \text { App. Res. } \\ 0.91( & 3.00) & 212.00 & 6.10( & 20.00) & 113.00 \\ 1.22( & 4.00) & 157.00 & 9.14( & 30.00) & 181.00 \\ 1.83( & 6.00) & 127.00 & 12.19( & 40.00) & 171.00 \\ 2.44( & 8.00) & 127.00 & 18.29( & 60.00) & 116.00 \\ 3.05( & 10.00) & 120.00 & 24.38( & 80.00) & 117.00 \\ 4.27( & 14.00) & 75.00 & 30.48( & 100.00) & 159.00 \\ 3.05( & 10.00) & 125.00 & 42.67(140.00) & 62.00 \\ 4.27( & 14.00) & 75.90 & 30.48(100.00) & 94.00 \\ & & & 42.67(140.00) & 80.00\end{array}$

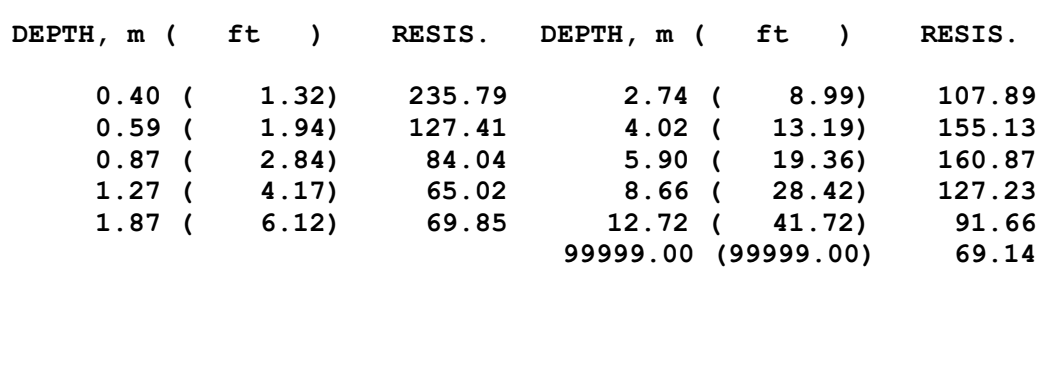



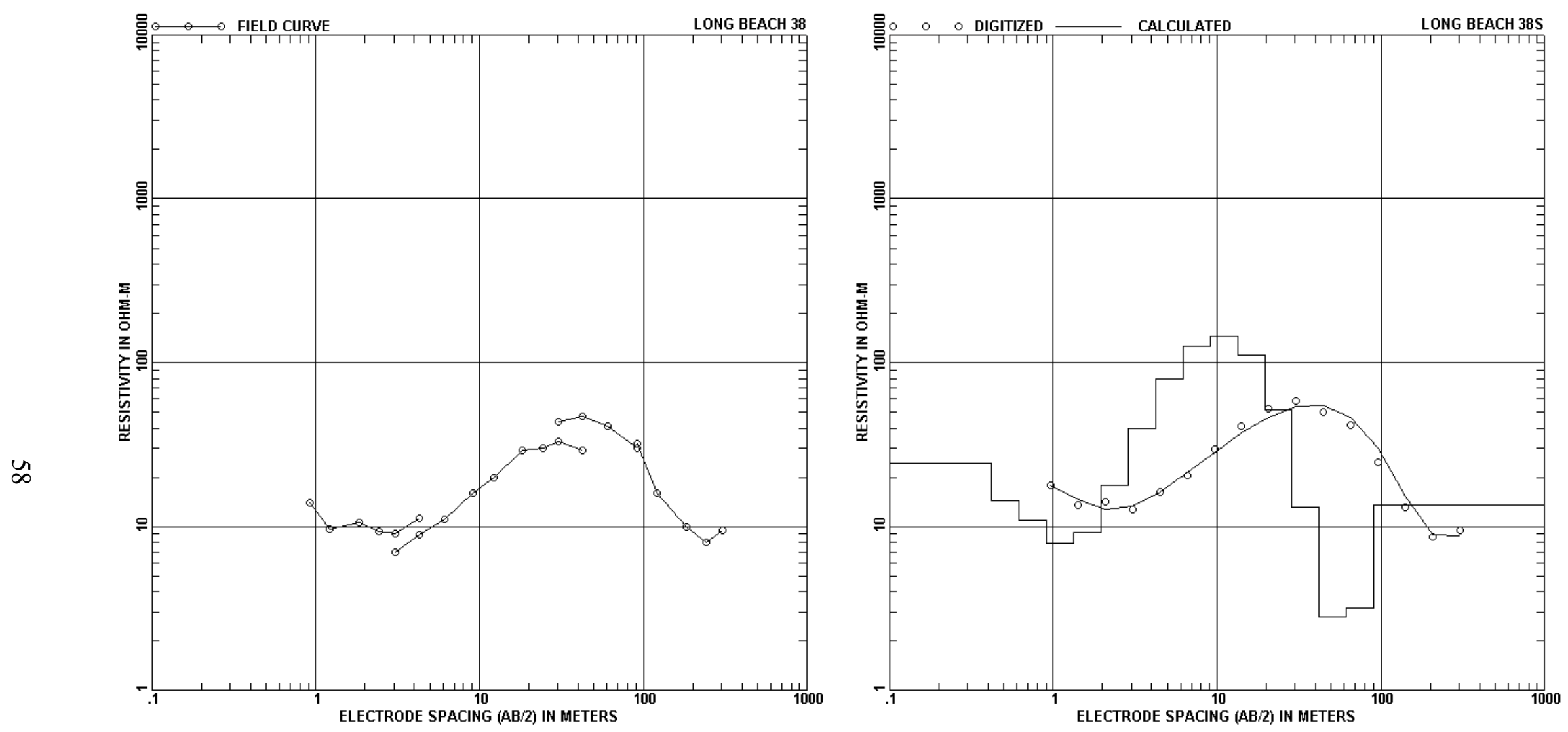

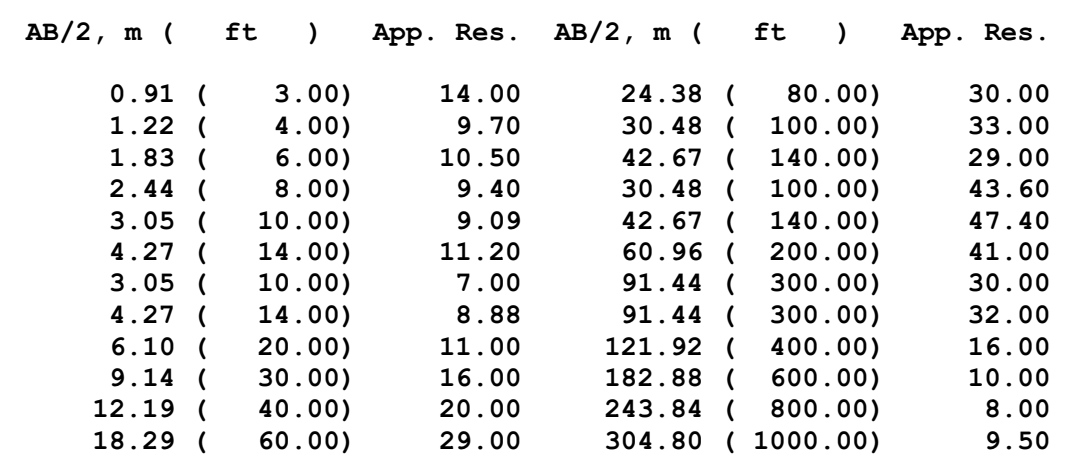

DEPTH， m ( ft )

\begin{tabular}{|c|c|}
\hline .42 & 1. \\
\hline 0.6 & $2.03)$ \\
\hline .91 & $2.98)$ \\
\hline 1.33 & $4.37)$ \\
\hline 1.96 & $6.42)$ \\
\hline 2.87 & $9.42)$ \\
\hline 1.2 & $.3 .83)$ \\
\hline 6.19 & $20.30)$ \\
\hline
\end{tabular}

\begin{abstract}
RESIS.
\end{abstract}

24.11

14.27

10.90

7.91

9.17

17.71

39.61

79.68
PTH, m ( ft )

$9.08(29.80)$ $13.33(43.74)$ 19.57 ( 64.20)

28.72 ( 94.23)

42.16 ( 138.32)

61.88 ( 203.02$)$ 99999.00 (99999.00)
RESIS.

126.39

145.22

111.73

13.15

2.79

3.17

13.55 

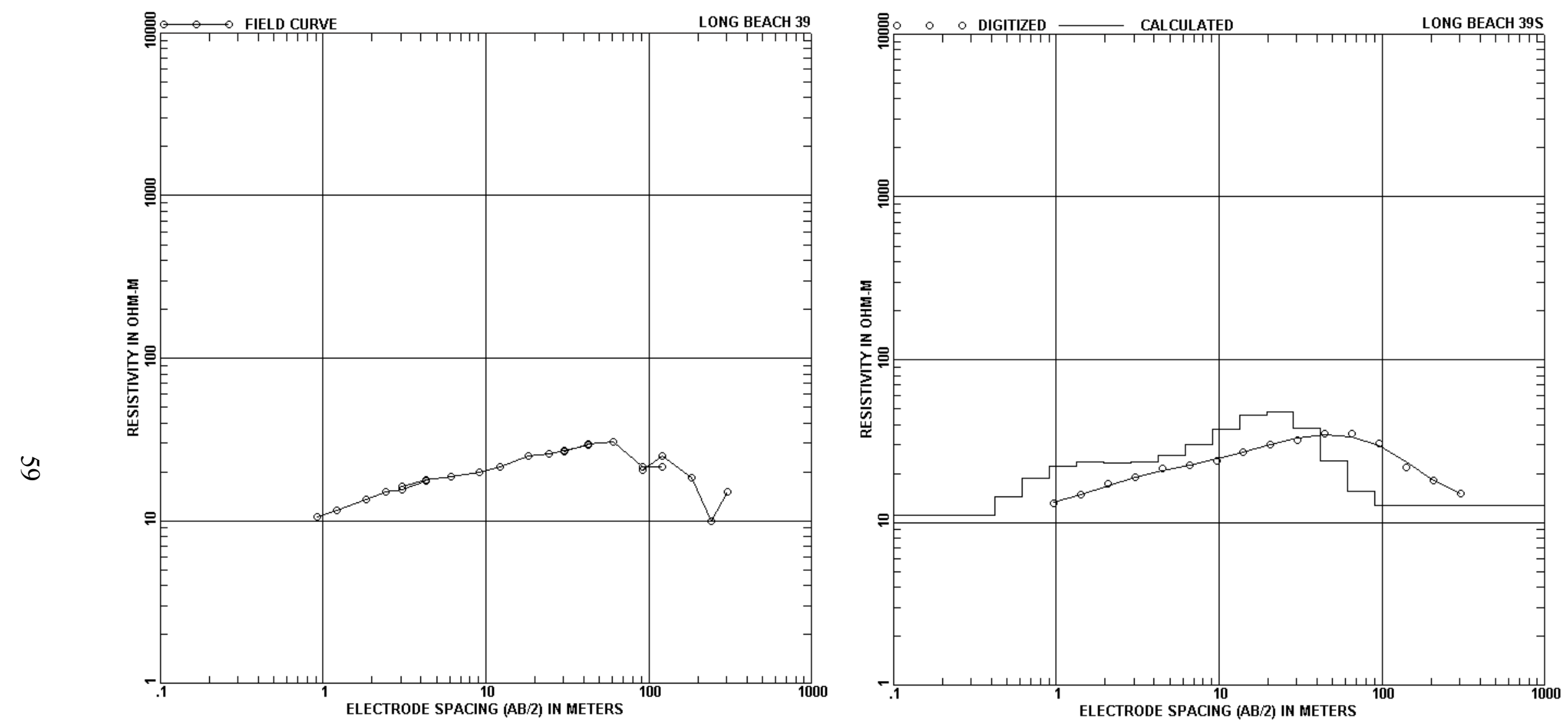

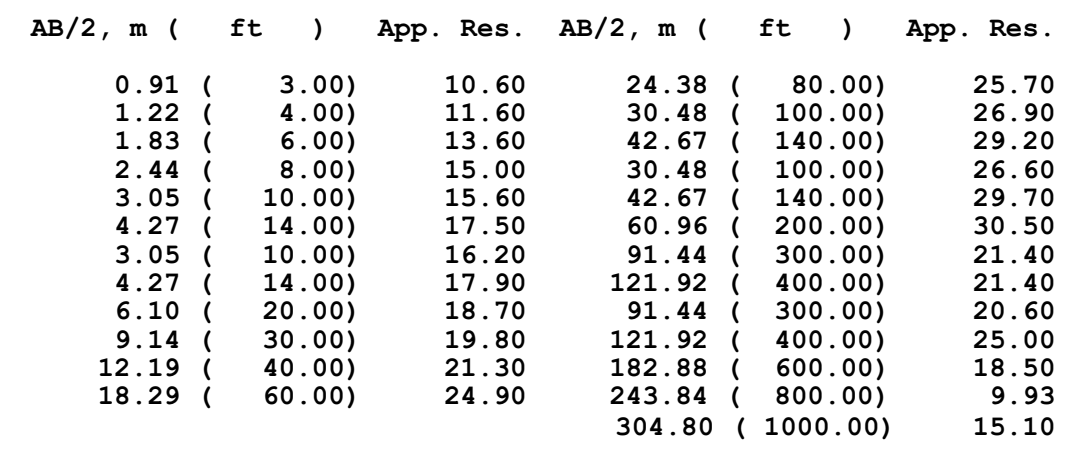

\begin{tabular}{|c|c|c|c|c|c|}
\hline DEPTH， m ( & ft ) & RESIS. & DEPTH， m ( & ft & RESIS. \\
\hline 0.42 & $1.38)$ & 10.99 & 9.08 & $29.80)$ & 30.17 \\
\hline 0.62 & $2.03)$ & 14.44 & 13.33 & $43.74)$ & 37.22 \\
\hline 0.91 & $2.98)$ & 18.56 & 19.57 & $64.20)$ & 45.32 \\
\hline 1.33 & $4.37)$ & 21.98 & 28.72 & $94.23)$ & 47.48 \\
\hline 1.96 & $6.42)$ & 23.36 & 42.16 & $(138.32)$ & 37.98 \\
\hline 2.87 & $9.42)$ & 23.12 & 61.88 & $(203.02)$ & 23.99 \\
\hline 4.22 & $13.83)$ & 23.42 & 90.83 & $(298.00)$ & 15.43 \\
\hline 6.19 & $20.30)$ & 25.69 & 99999.00 & $(99999.00)$ & 12.71 \\
\hline
\end{tabular}



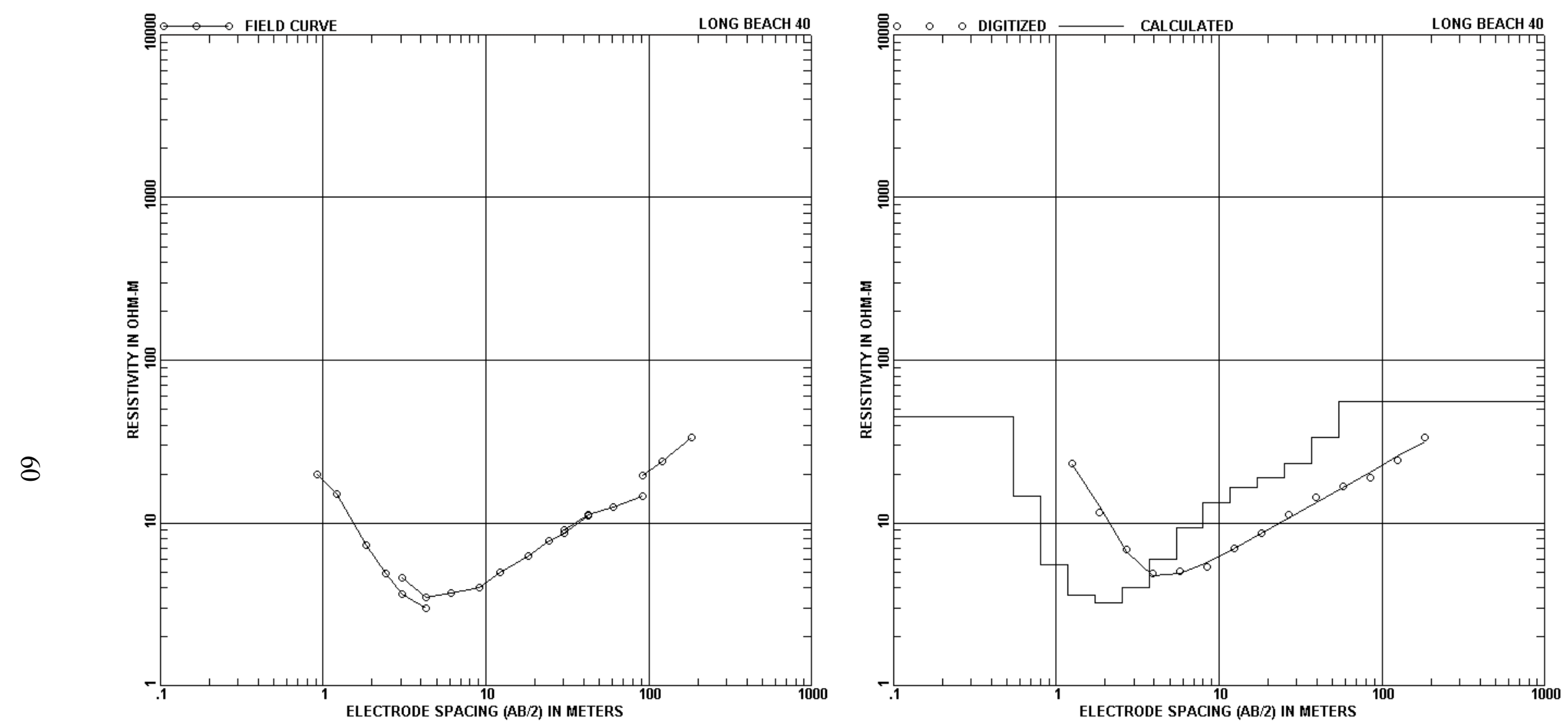

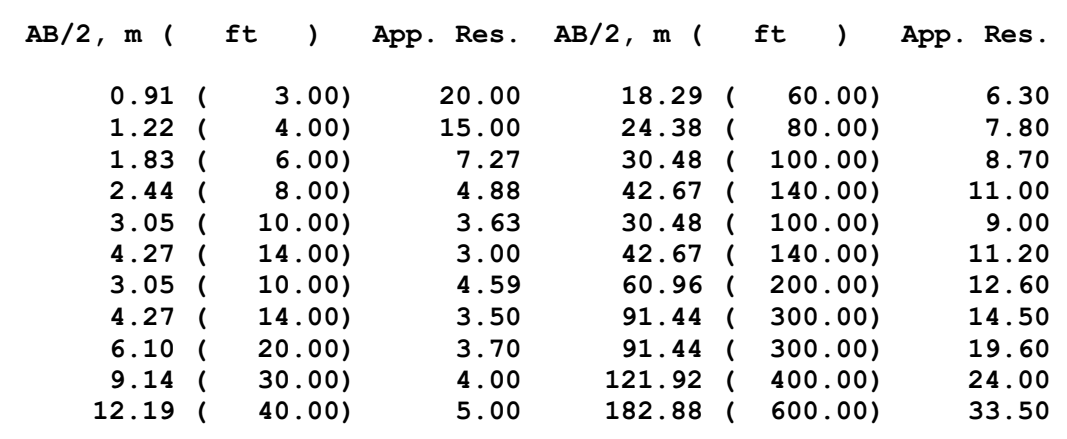

\begin{tabular}{|c|c|c|c|c|c|}
\hline DEPTH， m ( & ft & RESIS. & DEPTH， m & ft & RESIS. \\
\hline 0.541 & $1.79)$ & 45.04 & 8.00 & $26.24)$ & 9.31 \\
\hline $0.80 i$ & $2.62)$ & 14.57 & 11.74 & $38.52)$ & 13.37 \\
\hline 1.17 ( & $3.85)$ & 5.54 & 17.23 & $56.54)$ & 16.64 \\
\hline $1.72 i$ & $5.65)$ & 3.61 & 25.30 & $82.99)$ & 18.94 \\
\hline $2.53 i$ & $8.30)$ & 3.23 & 37.13 & $121.81)$ & 23.12 \\
\hline 3.71 & $12.18)$ & 3.99 & 54.50 & $(178.80)$ & 33.70 \\
\hline $5.45 i$ & $17.88)$ & 5.99 & 99999.00 & $(99999.00)$ & 55.91 \\
\hline
\end{tabular}



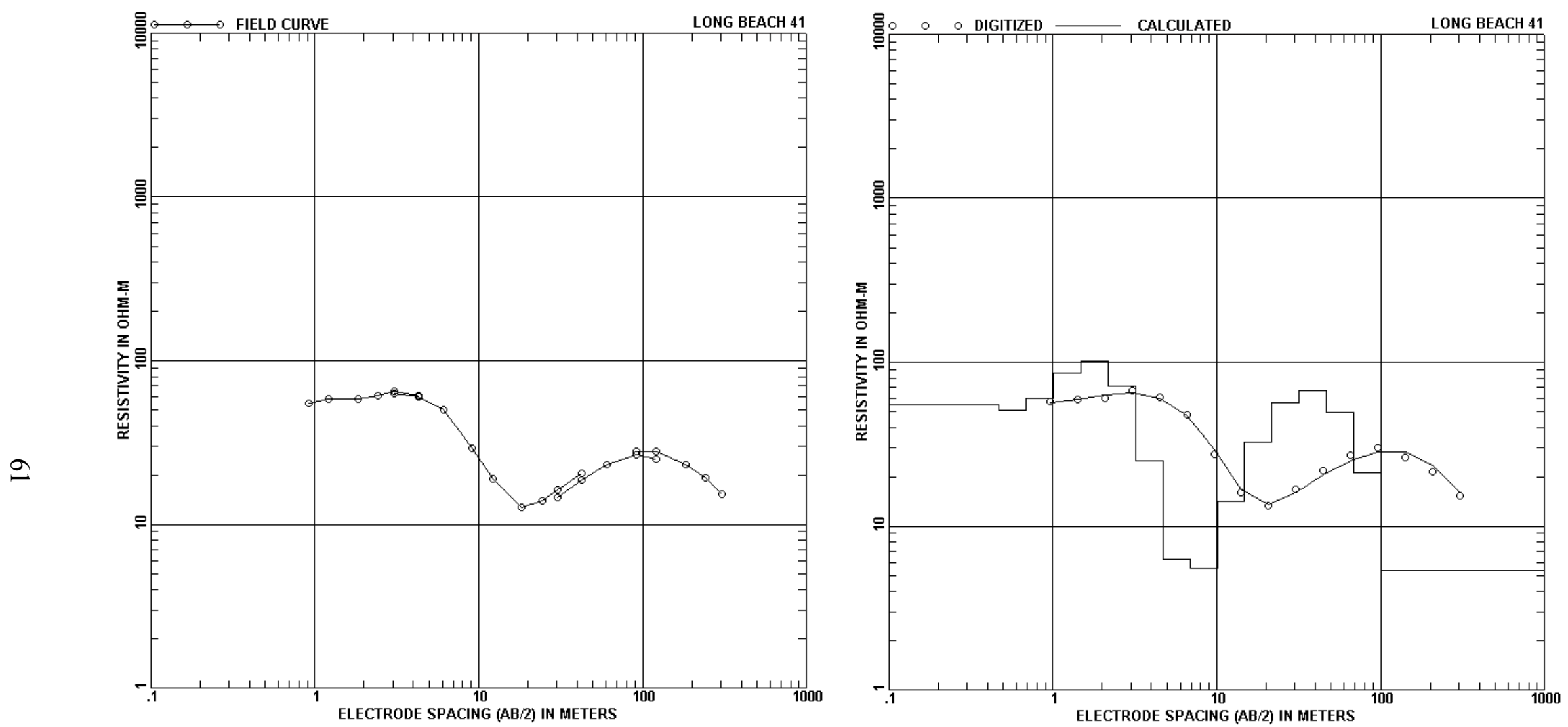

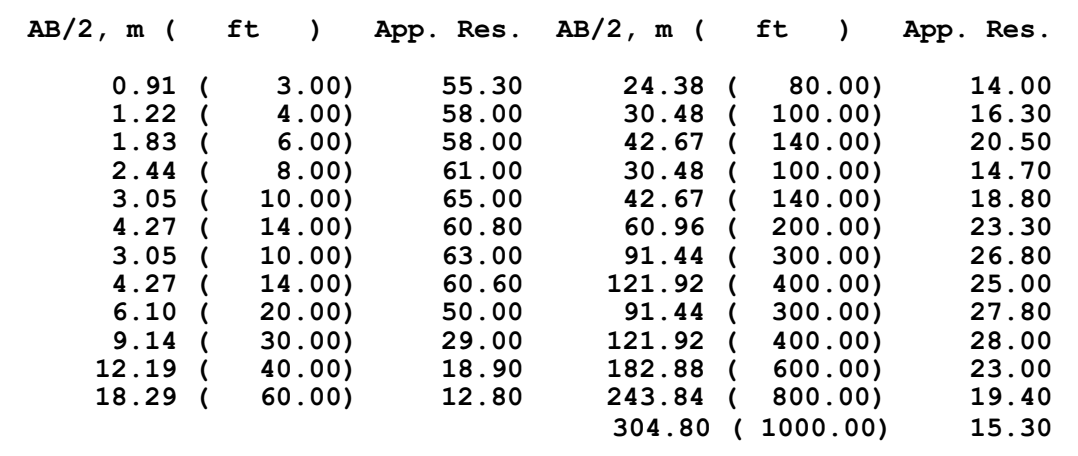

\begin{tabular}{|c|c|c|c|c|c|}
\hline DEPTH， m ( & ft ) & RESIS. & DEPTH， m & ft & RESIS. \\
\hline 0.47 & $1.54)$ & 55.25 & 10.09 & $33.11)$ & 5.51 \\
\hline 0.69 & $2.26)$ & 50.73 & 14.81 & $48.60)$ & 14.09 \\
\hline 1.01 & $3.31)$ & 60.43 & 21.74 & $71.34)$ & 32.75 \\
\hline 1.48 & $4.86)$ & 86.12 & 31.91 & $(104.71)$ & 56.62 \\
\hline 2.17 & $7.13)$ & 101.33 & 46.84 & ( 153.69$)$ & 67.05 \\
\hline 3.19 & $10.47)$ & 71.69 & 68.76 & ( 225.58) & 49.56 \\
\hline 4.68 & $15.37)$ & 25.10 & 100.92 & (331.11) & 20.99 \\
\hline 6.88 & $22.56)$ & 6.25 & 99999.00 & $(99999.00)$ & 5.35 \\
\hline
\end{tabular}



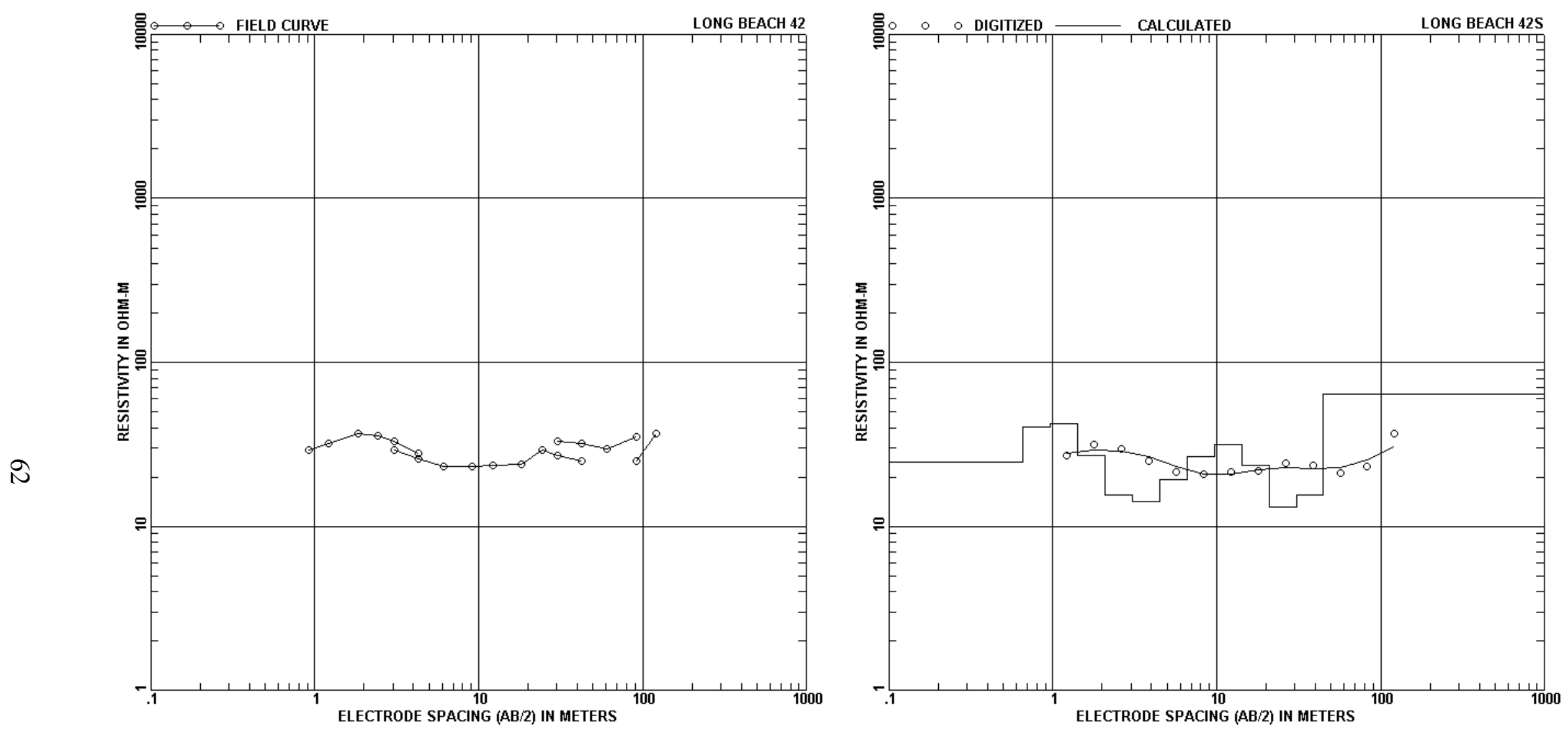

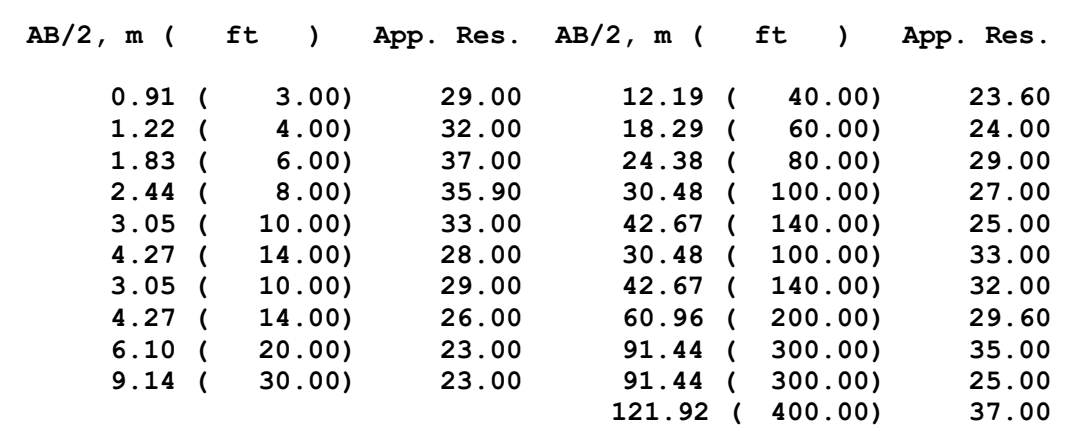

\begin{tabular}{|c|c|c|c|c|c|}
\hline DEPTH， m ( & ft ) & RESIS. & DEPTH， $\mathrm{m}$ ( & ft & RESIS. \\
\hline 0.661 & $2.16)$ & 24.54 & 6.58 & $21.60)$ & 19.19 \\
\hline $0.97 i$ & $3.17)$ & 40.47 & 9.66 & $31.70)$ & 26.81 \\
\hline $1.42 i$ & $4.65)$ & 42.04 & 14.18 & $46.54)$ & 31.66 \\
\hline $2.08 i$ & $6.83)$ & 26.83 & 20.82 & $68.31)$ & 23.61 \\
\hline $3.06 i$ & $10.03)$ & 15.59 & 30.56 & $100.26)$ & 13.13 \\
\hline 4.49 i & $14.72)$ & 14.21 & 44.85 & $(147.16)$ & 15.45 \\
\hline & & & 99999.00 & $(99999.00)$ & 63.92 \\
\hline
\end{tabular}



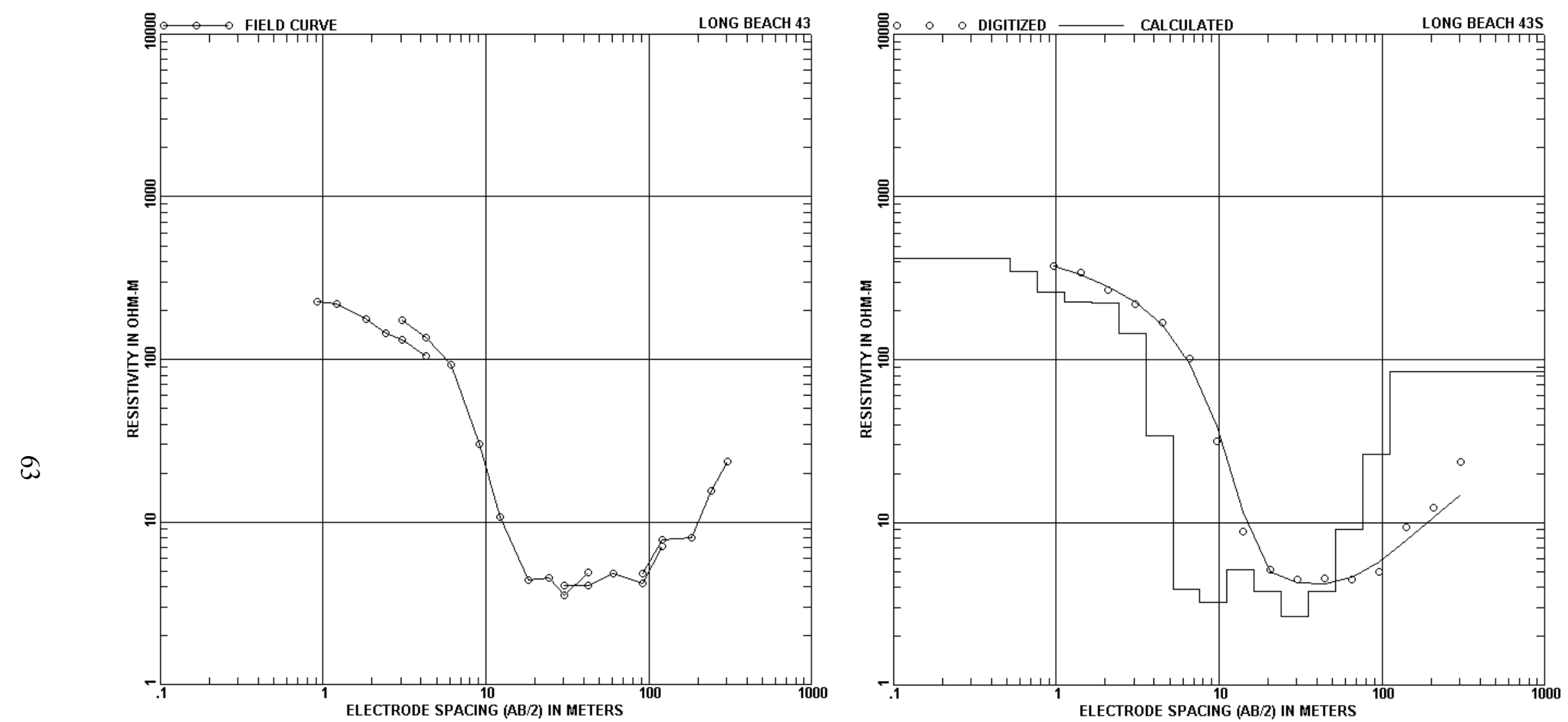

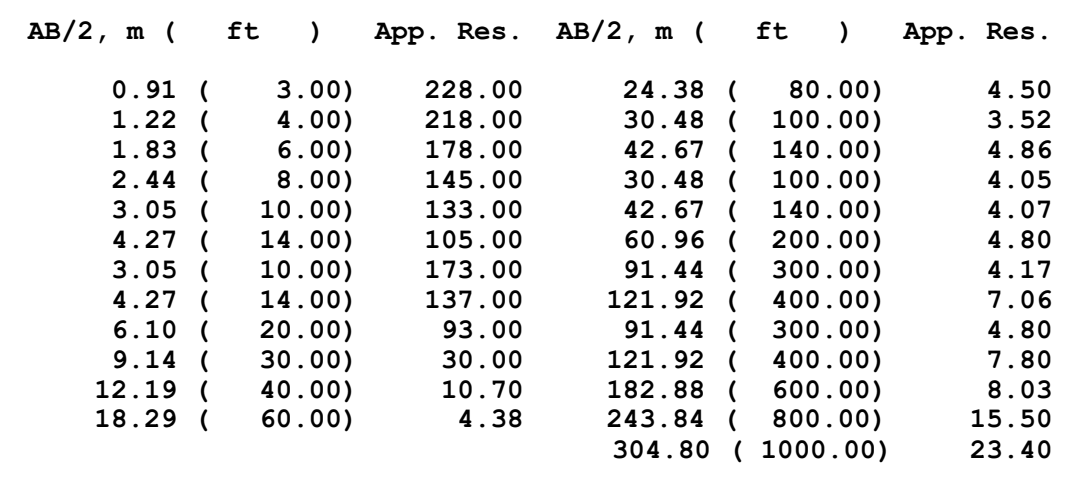

DEPTH， m ( ft )

$\begin{array}{rrr}0.52 & ( & 1.71) \\ 0.76 & ( & 2.51) \\ 1.12 & ( & 3.68) \\ 1.65 & ( & 5.40) \\ 2.42 & ( & 7.93) \\ 3.55 & ( & 11.63) \\ 5.20 & ( & 17.08) \\ 7.64 & ( & 25.06)\end{array}$

RESIS.

$\begin{array}{rrlr}420.66 & 11.21 & (\quad 36.79) & 3.22 \\ 349.23 & 16.46(154.00) & 5.15 \\ 260.43 & 24.16(19.26) & 3.78 \\ 225.03 & 35.46(116.34) & 2.66 \\ 221.11 & 52.05(170.76) & 3.74 \\ 144.14 & 76.40 & (250.65) & 9.13 \\ 34.06 & 112.14 & (367.90) & 26.30 \\ 3.86 & 99999.00 & (99999.00) & 84.17\end{array}$



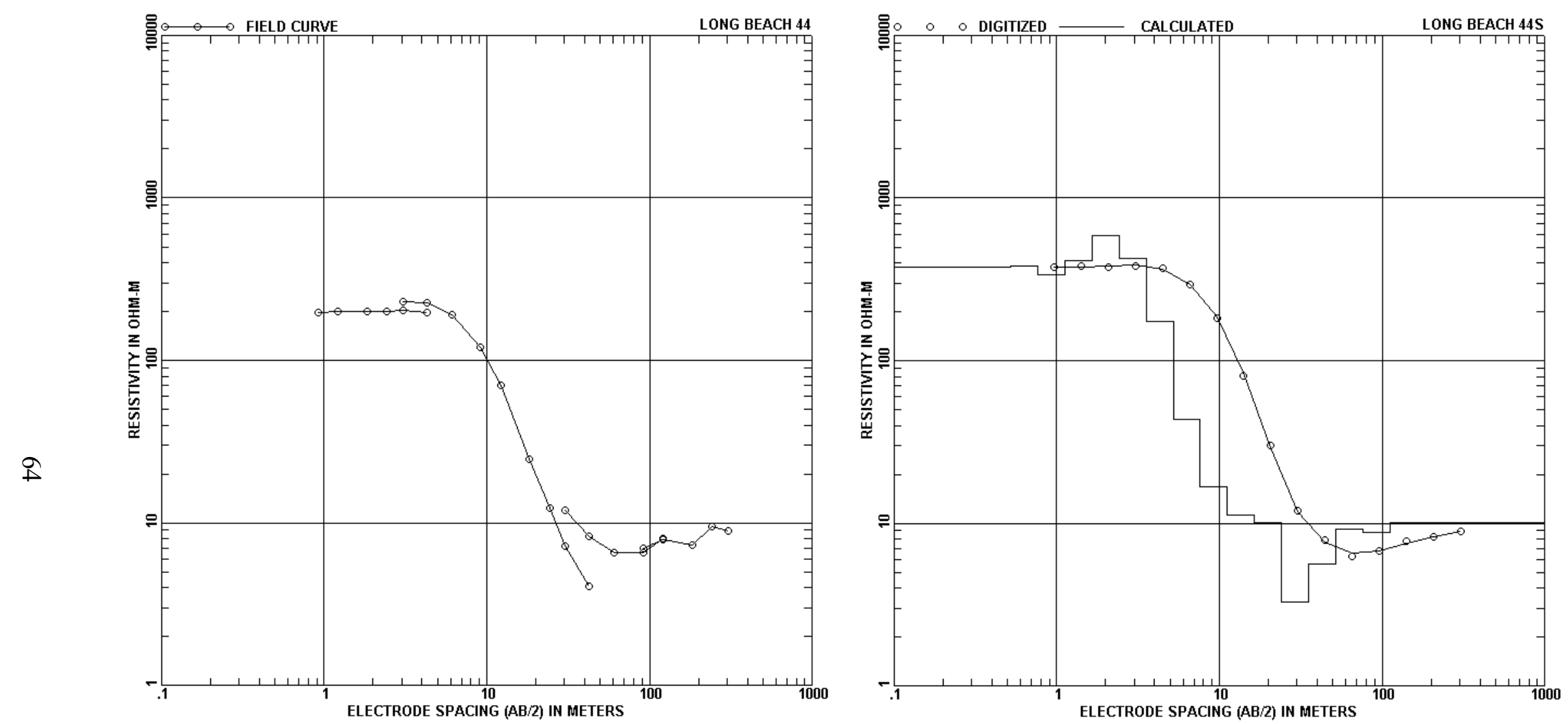

\begin{tabular}{|c|c|c|c|c|c|}
\hline $\mathrm{AB} / 2, \mathrm{~m}$ ( & ) & p. Res. & $\mathrm{B} / 2, \mathrm{~m}$ ( & ft & Res. \\
\hline 0.91 & $3.00)$ & 198.00 & 24.38 & $80.00)$ & 12.30 \\
\hline 1.22 & $4.00)$ & 200.00 & 30.48 & $100.00)$ & 7.19 \\
\hline 1.83 & $6.00)$ & 200.00 & 42.67 & $140.00)$ & 4.08 \\
\hline 2.44 & $8.00)$ & 200.00 & 30.48 & $100.00)$ & 12.00 \\
\hline 3.05 & $10.00)$ & 202.00 & 42.67 & $140.00)$ & 8.20 \\
\hline 4.27 & $14.00)$ & 198.00 & 60.96 & $200.00)$ & 6.54 \\
\hline 3.05 & $10.00)$ & 231.00 & 91.44 & $300.00)$ & 6.54 \\
\hline 4.27 & $14.00)$ & 226.00 & 121.92 & $400.00)$ & 7.98 \\
\hline 6.10 & $20.00)$ & 190.00 & 91.44 & $300.00)$ & 6.93 \\
\hline 9.14 & $30.00)$ & 120.00 & 121.92 & $400.00)$ & 7.93 \\
\hline 12.19 & $40.00)$ & 70.00 & 182.88 & $600.00)$ & 7.35 \\
\hline 18.29 & $60.00)$ & 24.60 & 243.84 & $800.00)$ & 9.45 \\
\hline & & & 304.80 & $1000.00)$ & 8.88 \\
\hline
\end{tabular}

DEPTH, m ( ft )

$\begin{array}{rrr}0.52 & ( & 1.71) \\ 0.76 & ( & 2.51) \\ 1.12 & ( & 3.68) \\ 1.65 & ( & 5.40) \\ 2.42 & ( & 7.93) \\ 3.55 & ( & 11.63) \\ 5.20 & ( & 17.08) \\ 7.64 & ( & 25.06)\end{array}$
RESIS. DEPTH, m ( ft )

RESIS.

\begin{tabular}{|c|c|c|c|}
\hline 373.36 & 11.21 & $36.79)$ & 16.79 \\
\hline 382.56 & 16.46 & $54.00)$ & 11.22 \\
\hline 339.53 & 24.16 & $79.26)$ & 10.11 \\
\hline 410.65 & 35.46 & $116.34)$ & 3.30 \\
\hline 591.50 & 52.05 & $170.76)$ & 5.61 \\
\hline 427.22 & 76.40 & $250.65)$ & 9.26 \\
\hline 173.83 & 112.14 & $367.90)$ & 8.77 \\
\hline 43.26 & 99999.00 & (99999.00) & 10.07 \\
\hline
\end{tabular}



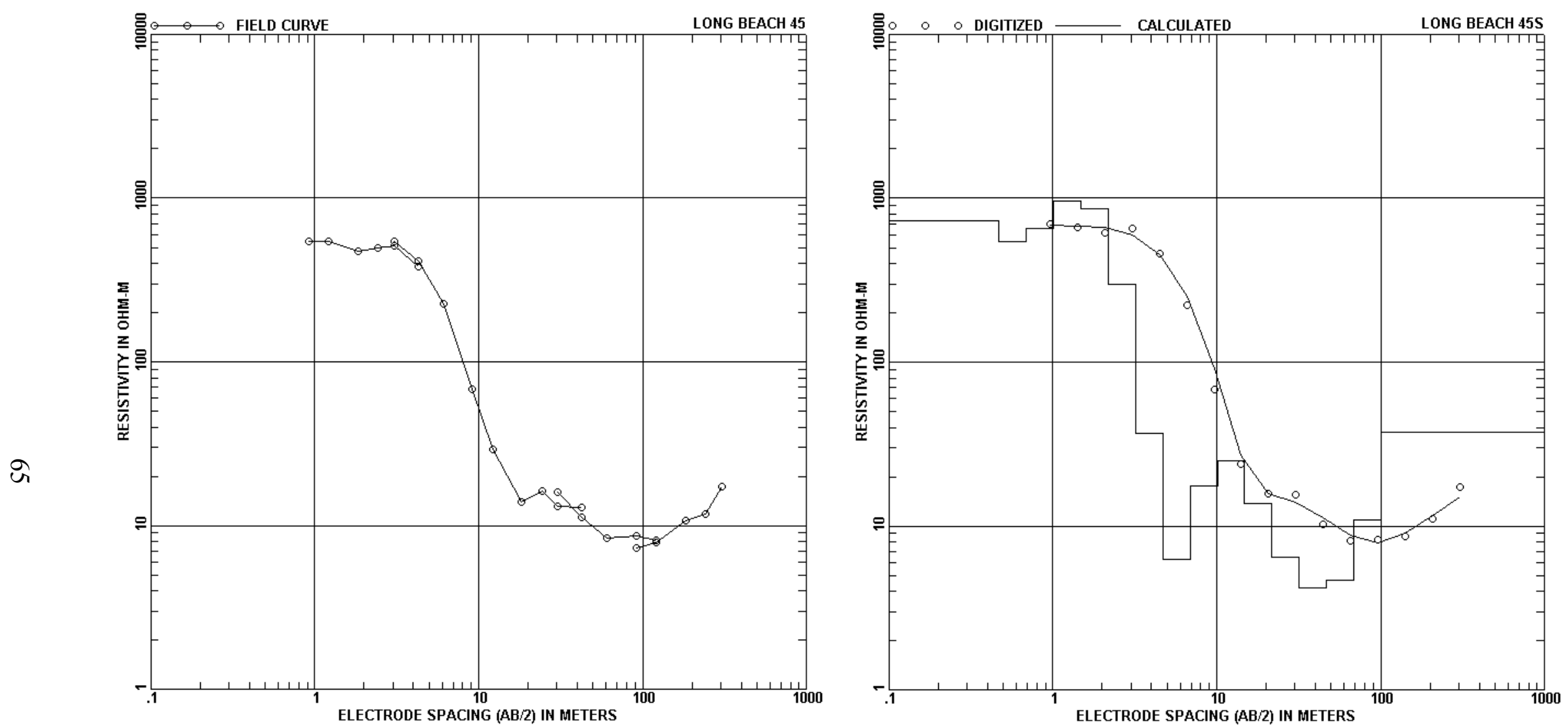

\begin{tabular}{|c|c|c|c|c|c|}
\hline $\mathrm{AB} / 2, \mathrm{~m}$ ( & ) & p. Res. & $/ 2, \mathrm{~m}($ & ft & Res. \\
\hline 0.91 & $3.00)$ & 543.00 & 24.38 & $80.00)$ & 16.20 \\
\hline 1.22 & $4.00)$ & 545.00 & 30.48 & $100.00)$ & 13.10 \\
\hline 1.83 & $6.00)$ & 477.00 & 42.67 & $140.00)$ & 12.90 \\
\hline 2.44 & $8.00)$ & 498.00 & 30.48 & $100.00)$ & 16.00 \\
\hline 3.05 & $10.00)$ & 510.00 & 42.67 & $140.00)$ & 11.30 \\
\hline 4.27 & $14.00)$ & 383.00 & 60.96 & $200.00)$ & 8.45 \\
\hline 3.05 & $10.00)$ & 545.00 & 91.44 & $300.00)$ & 8.66 \\
\hline 4.27 & $14.00)$ & 415.00 & 121.92 & $400.00)$ & 8.13 \\
\hline 6.10 & $20.00)$ & 228.00 & 91.44 & $300.00)$ & 7.27 \\
\hline 9.14 & $30.00)$ & 68.00 & 121.92 & $400.00)$ & 7.85 \\
\hline 12.19 & $40.00)$ & 29.00 & 182.88 & $600.00)$ & 10.80 \\
\hline 18.29 & $60.00)$ & 13.90 & 243.84 & $800.00)$ & 11.80 \\
\hline & & & 304.80 & $1000.00)$ & 17.20 \\
\hline
\end{tabular}

DEPTH, m ( ft )

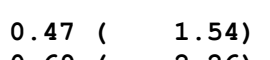

$0.69(2.26)$

1.01 (3.31)

$1.48(4.86)$

2.17 ( 7.13 )

$3.19(10.47)$

$4.68(15.37)$

6.88 (22.56)

R

RESIS.

DEPTH， m ( ft )

RESIS.

\begin{tabular}{|c|c|c|c|}
\hline 727.62 & 10.09 & 33.11) & 17.49 \\
\hline 43.8 & 14.81 & $48.60)$ & 24.93 \\
\hline 658.4 & 21.74 & $71.34)$ & 13.77 \\
\hline 966.4 & 31.91 & $104.71)$ & 6.46 \\
\hline 857.83 & 46.84 & $153.69)$ & 4.20 \\
\hline 299.74 & 68.76 & $225.58)$ & 4.68 \\
\hline 36.57 & 100.92 & $331.11)$ & 10.98 \\
\hline 6.31 & 9999.00 & $(99999.00)$ & 37.21 \\
\hline
\end{tabular}



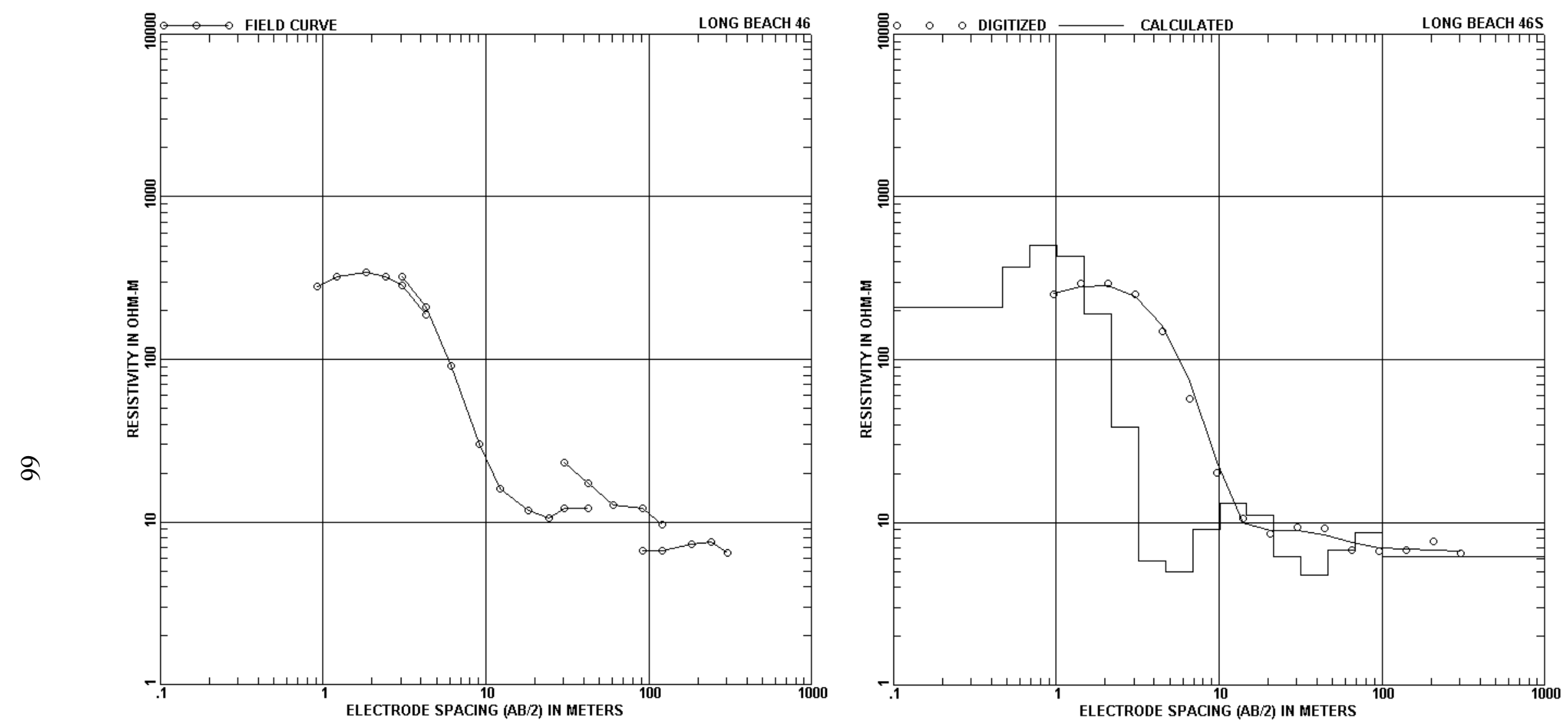

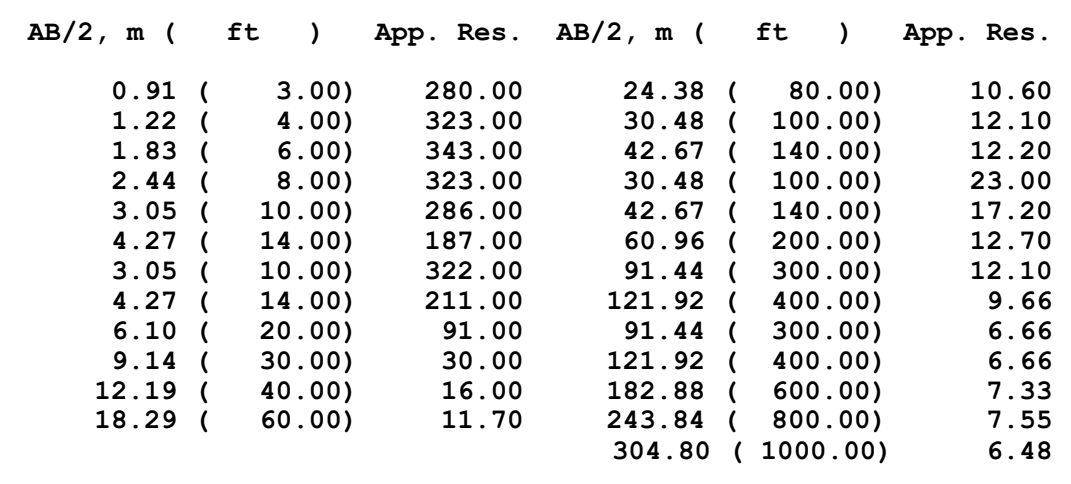

DEPTH, m ( ft )

\begin{tabular}{|c|c|}
\hline .47 & \\
\hline 0.69 & \\
\hline L. 01 & 3.31) \\
\hline 1.48 & $4.86)$ \\
\hline 2.17 & $7.13)$ \\
\hline .19 & $0.47)$ \\
\hline 68 & $5.37)$ \\
\hline 6.88 & $22.56)$ \\
\hline
\end{tabular}

\begin{abstract}
RESIS.
\end{abstract}
209.89

371.91

500.68

429.41

429.41
191.48

191.48

38.52

5.82
4.97

DEPTH, m ( ft )

RESIS.

10.09 ( 33.11$)$

8.99

14.81 ( 48.60$)$

11.08

31.91 ( 104.71$)$

6.14

$46.84(153.69)$

$68.76(225.58)$

4.76

4.76

6.79

8.65

6.17 

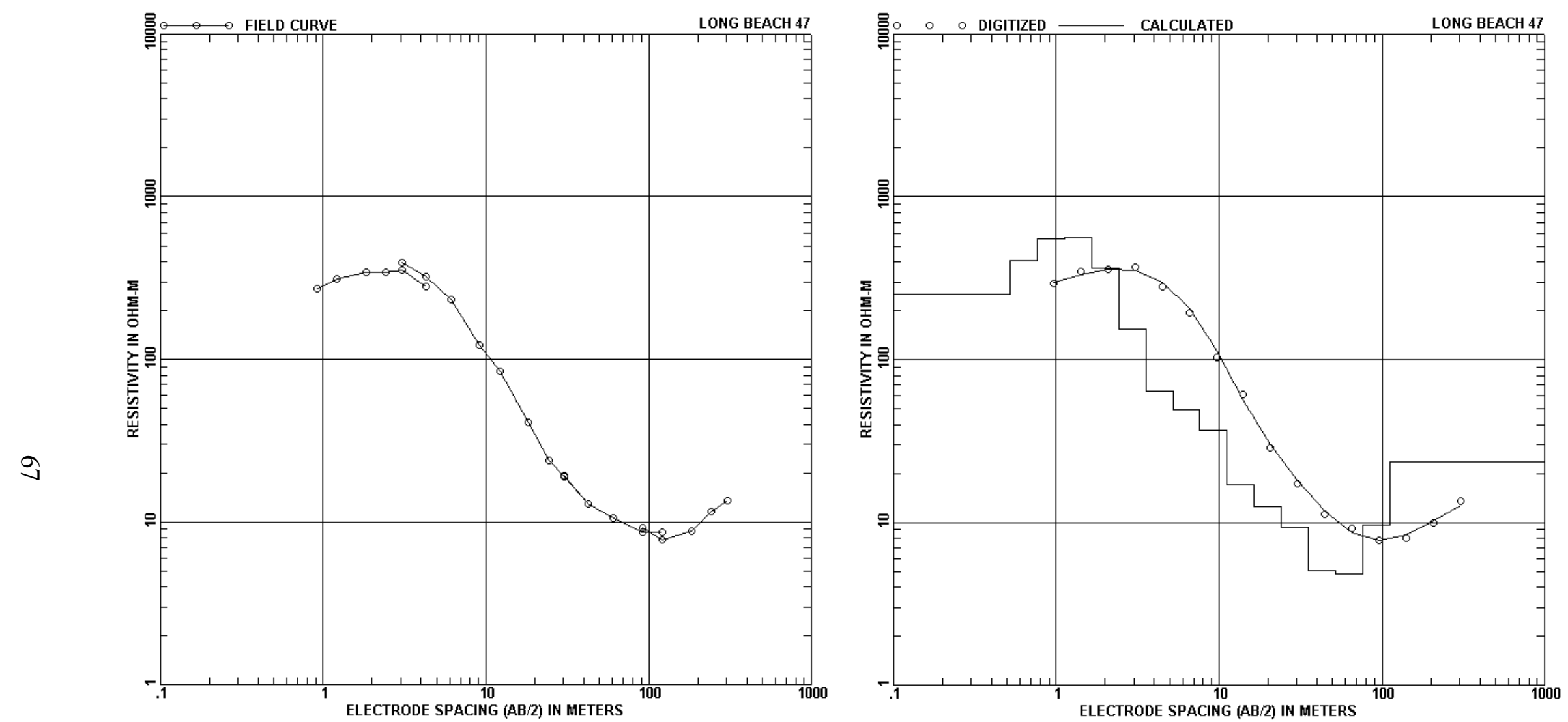

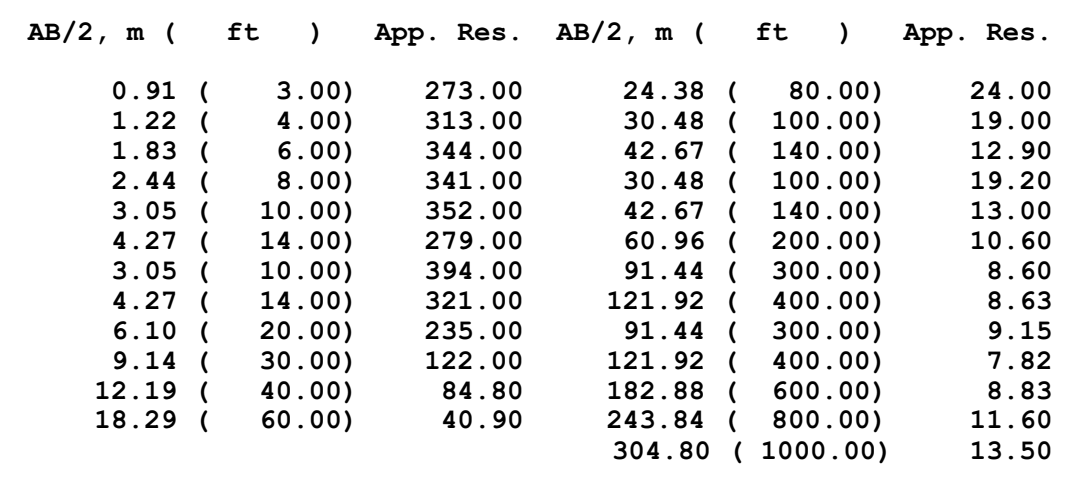

DEPTH， m ( ft )

\begin{tabular}{|c|c|}
\hline .52 & 1. \\
\hline 0.76 & $2.51)$ \\
\hline L. 12 & $3.68)$ \\
\hline 1.65 & $5.40)$ \\
\hline 2.42 & $7.93)$ \\
\hline 3.55 & (1. 63) \\
\hline 2 & $.7 .08)$ \\
\hline 7.64 & $25.06)$ \\
\hline
\end{tabular}

RESIS. DEPTH, $m$ ( ft )

RESIS.

$\begin{array}{rrlr}251.41 & 11.21( & (36.79) & 36.69 \\ 403.12 & 16.46( & 54.00) & 17.09 \\ 552.95 & 24.16(19.26) & 12.47 \\ 558.17 & 35.46(116.34) & 9.37 \\ 362.67 & 52.05(170.76) & 5.09 \\ 153.48 & 76.40(250.65) & 4.81 \\ 63.65 & 112.14(367.90) & 9.58 \\ 48.94 & 99999.00 & (99999.00) & 23.45\end{array}$



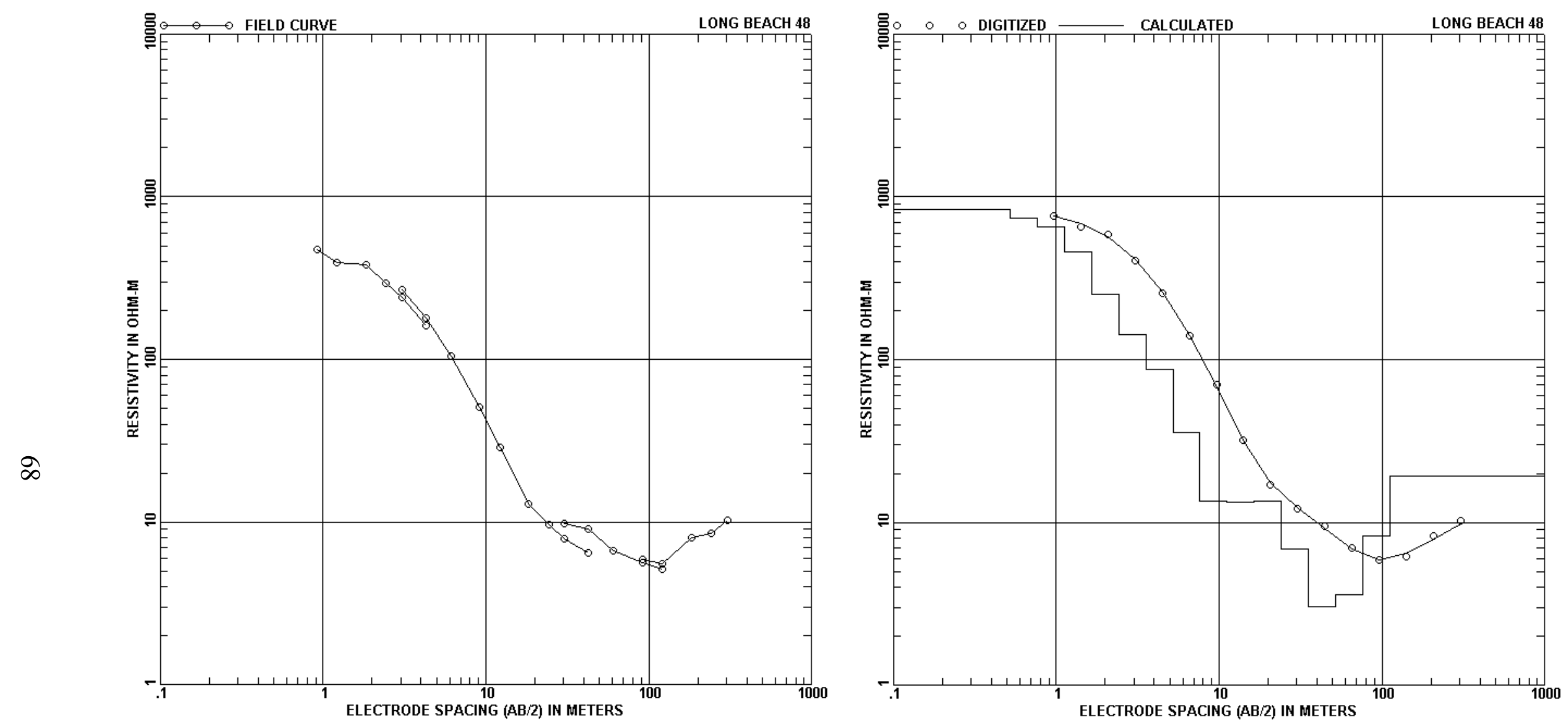

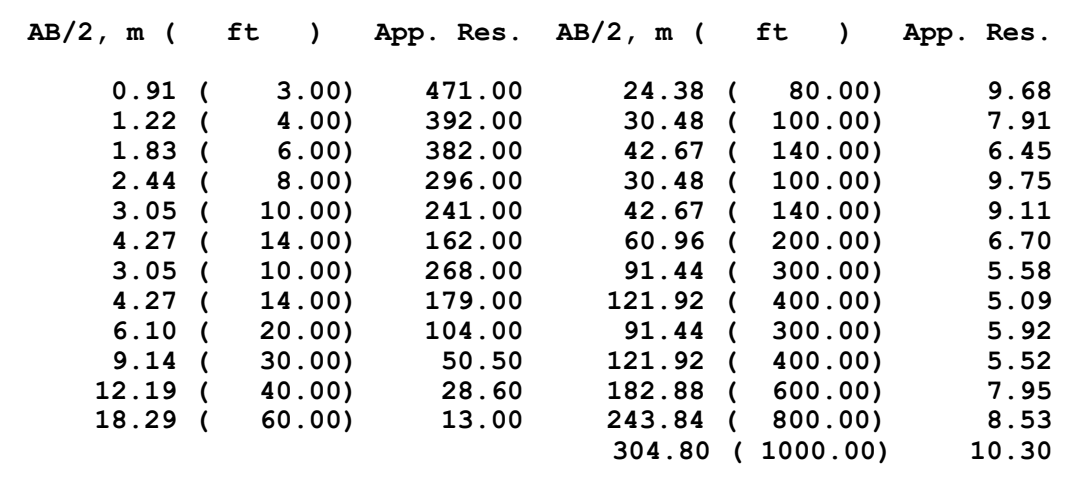

DEPTH， m ( ft )

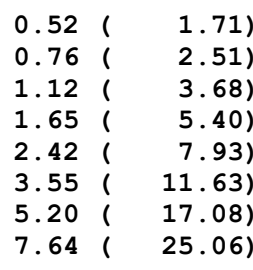
RESIS.

DEPTH, m ( ft )

RESIS.

\subsection{6}

741.81

655.00

462.75

253.50

141.57

87.13

35.75

$11.21(36.79)$

13.53

$24.16(79.26)$

13.61

$35.46(116.34)$

6.87

$52.05(170.76)$

$76.40(250.65)$

3.05

$12.14(367.90)$

3.61

8.21

19.38 

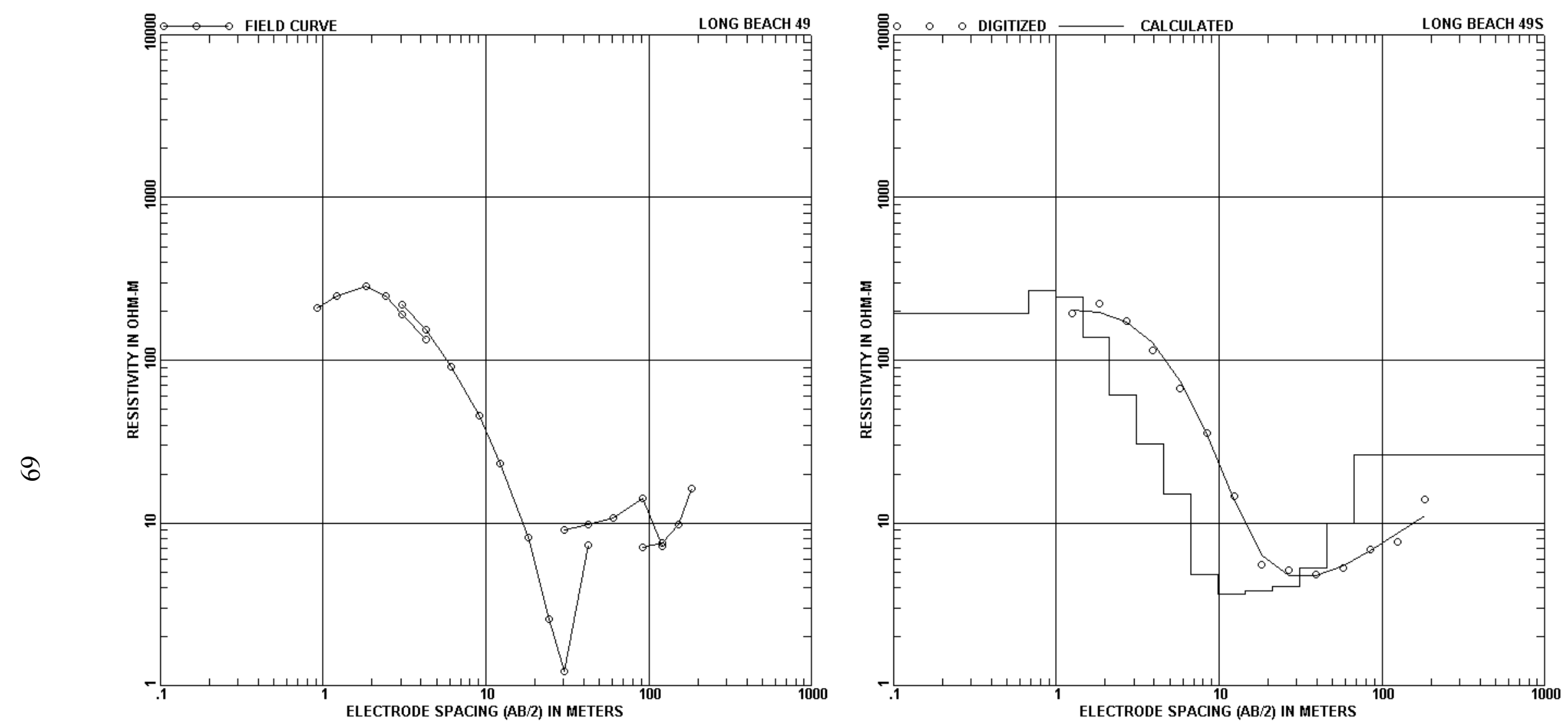

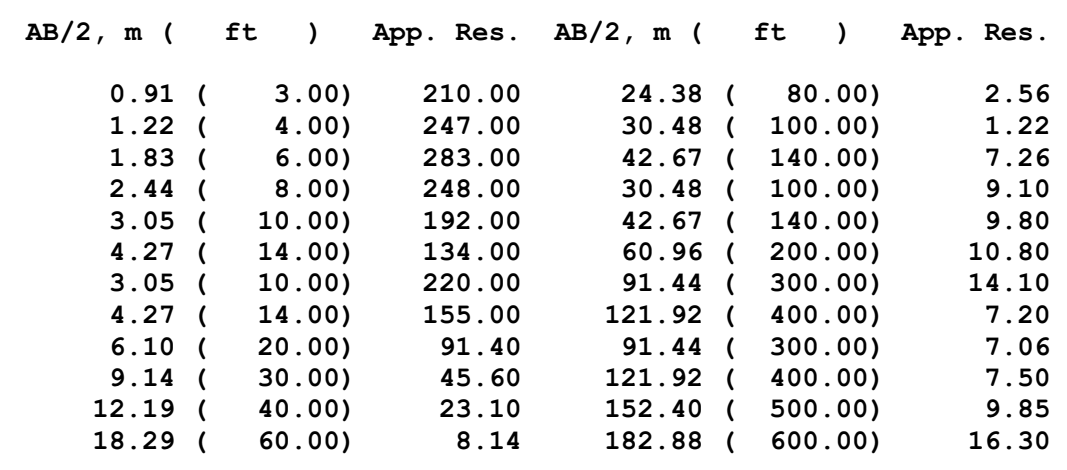

DEPTH, $m$ ( ft ) RESIS. DEPTH, $m($ ft

\begin{tabular}{|c|c|c|c|c|c|}
\hline 0.67 & 2.21) & 195.22 & 9.88 & $32.40)$ & 4.81 \\
\hline 0.99 & $3.24)$ & 268.73 & 14.50 & $47.56)$ & 3.65 \\
\hline 1.45 & $4.76)$ & 244.49 & 21.28 & $69.80)$ & 3.80 \\
\hline 2.13 & $6.98)$ & 138.47 & 31.23 & $102.46)$ & 4.06 \\
\hline 3.12 & $10.25)$ & 61.21 & 45.84 & $150.39)$ & 5.32 \\
\hline 4.58 & $15.04)$ & 30.80 & 67.28 & $220.74)$ & 10.00 \\
\hline 6.73 & $22.07)$ & 15.01 & 99999.00 & $(99999.00)$ & 26.38 \\
\hline
\end{tabular}



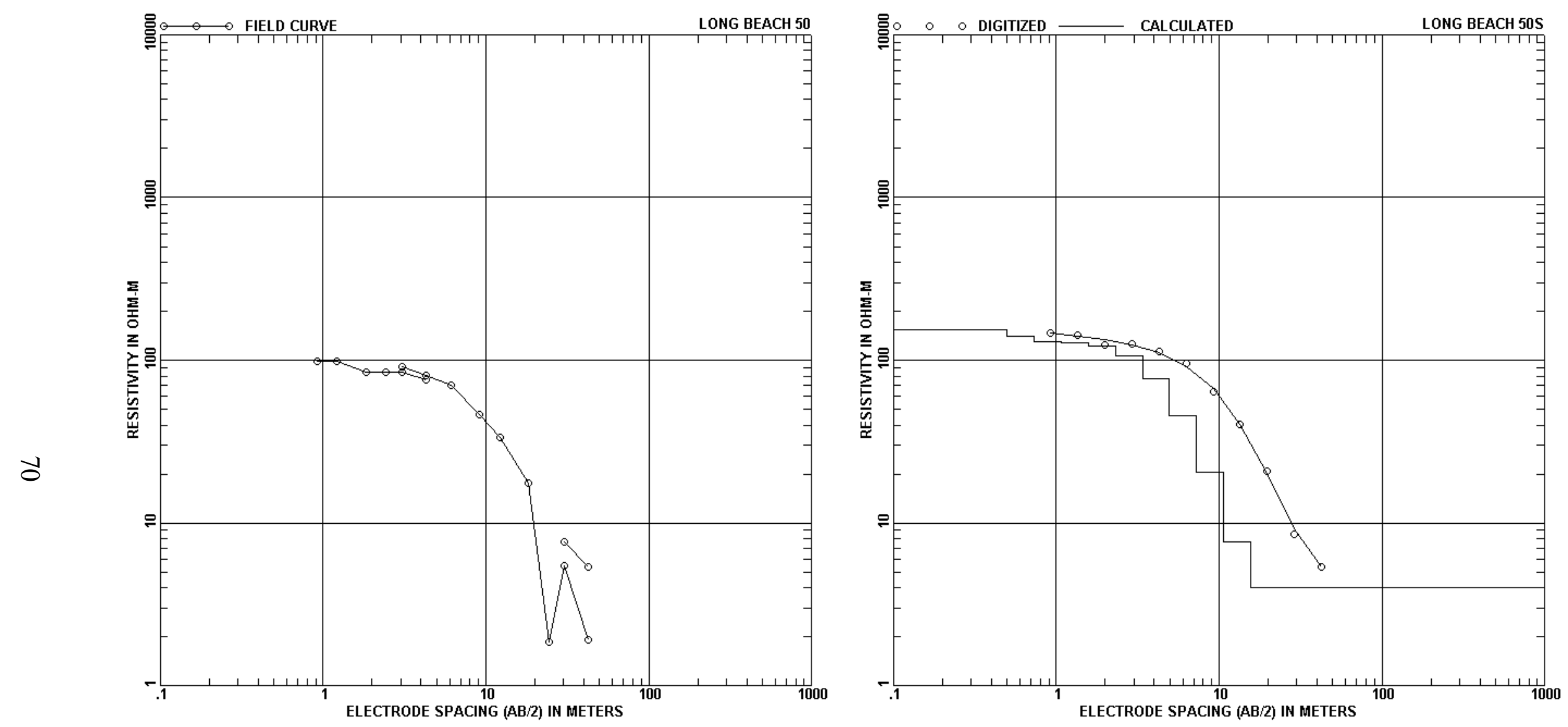

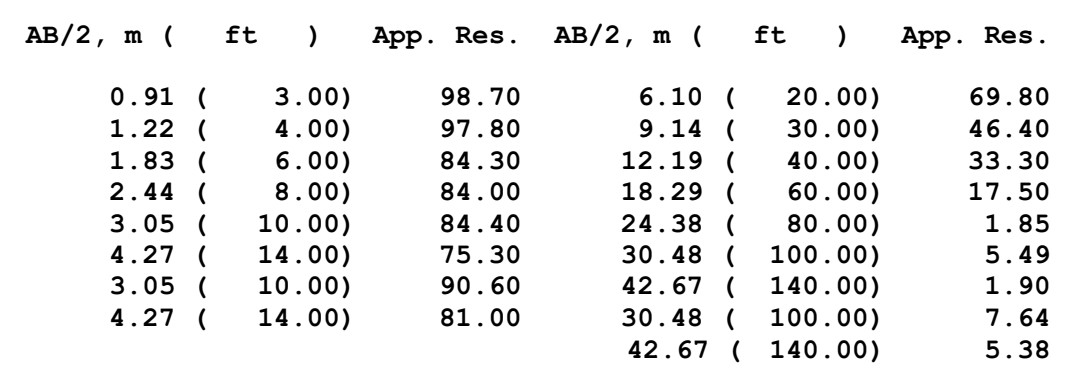

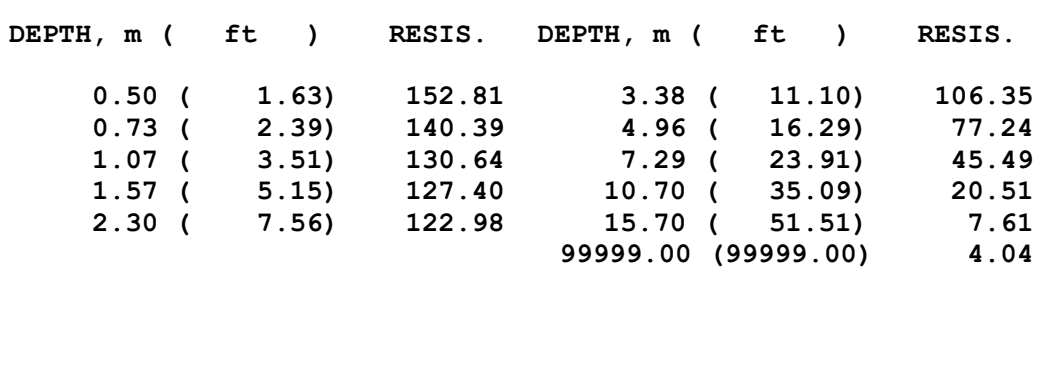



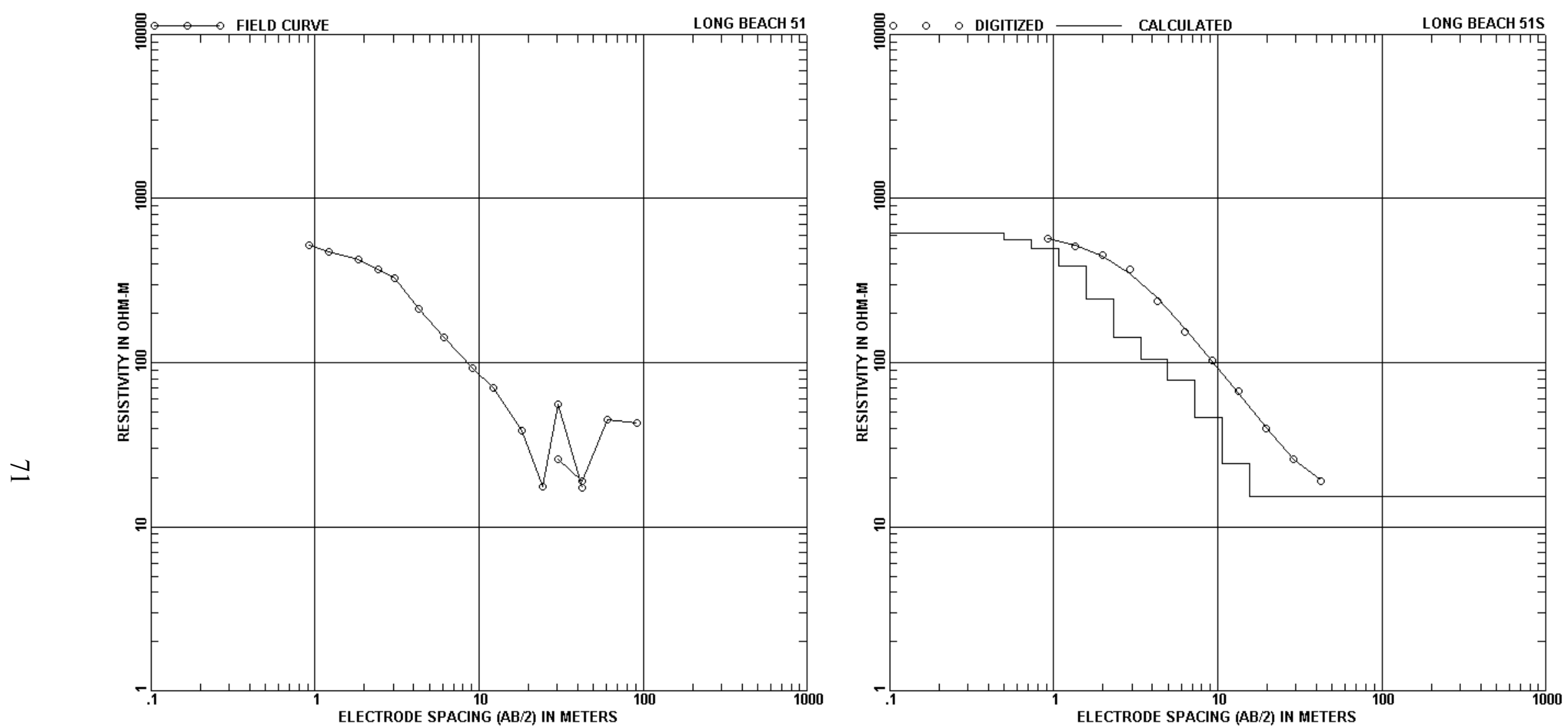

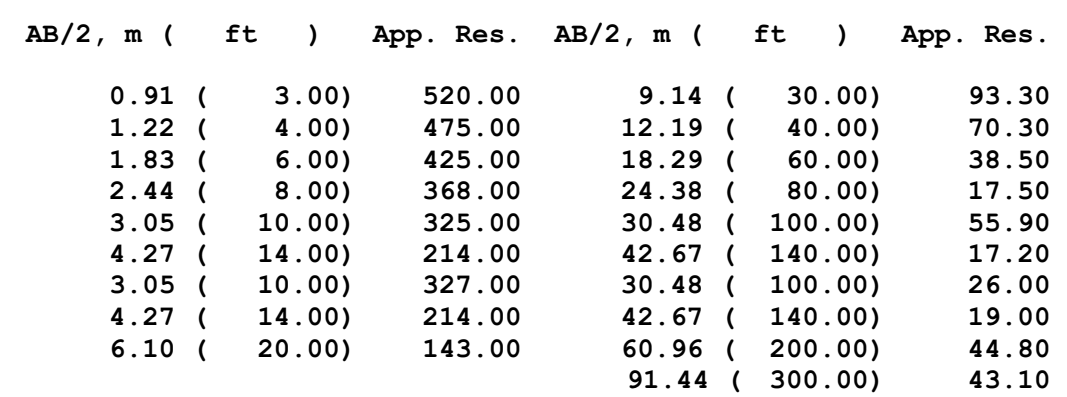

DEPTH, $m$ ( ft $)$ ( RESIS. DEPTH, $m$ (

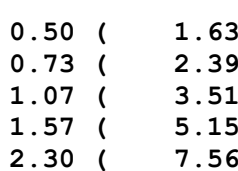

\subsection{9}

616.99
558.38

495.16

387.24

242.98

$3.38(11.10)$

$4.96(16.29)$

RESIS.

$$
\begin{aligned}
& 1.57 \\
& 2.30
\end{aligned}
$$

7. 56) 

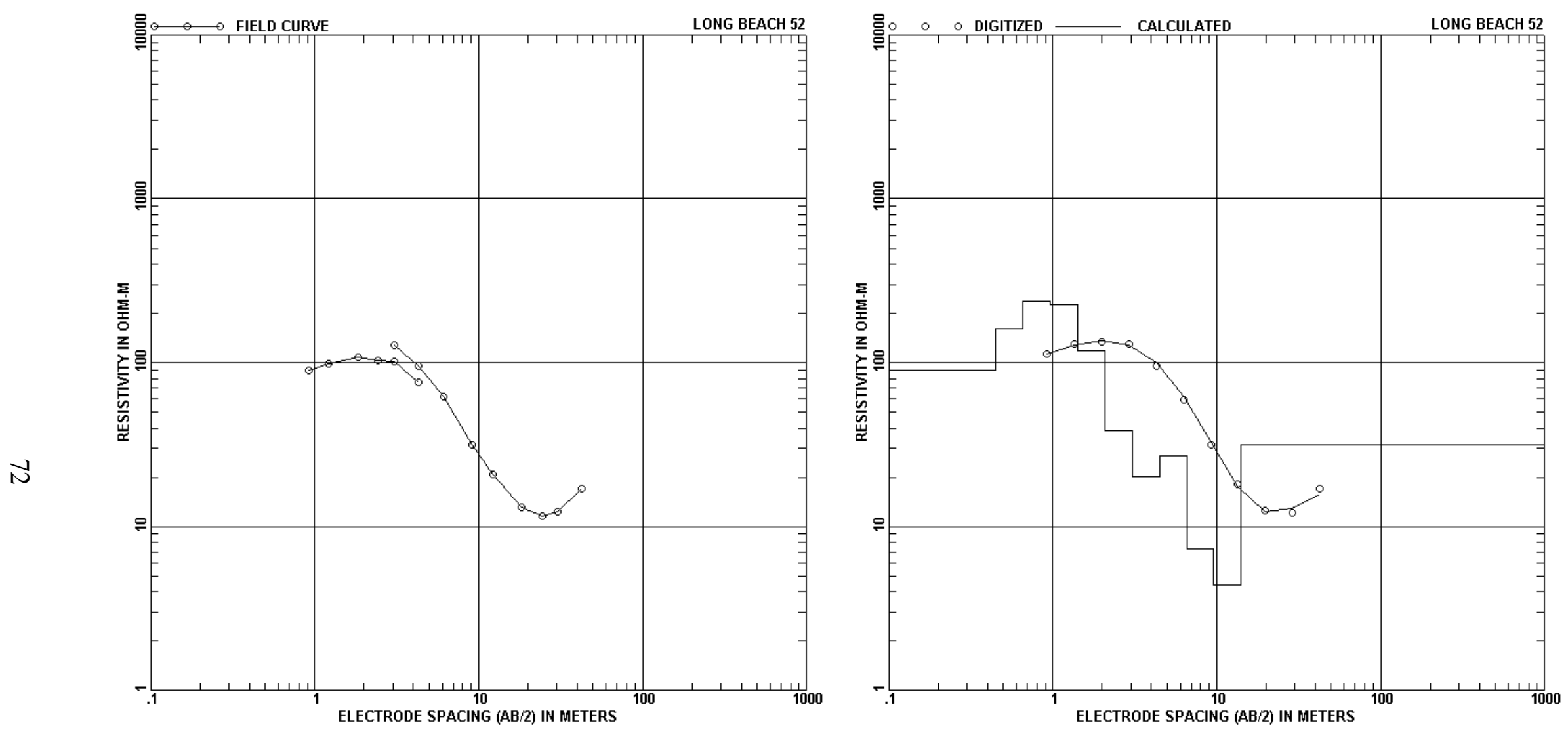

$\begin{array}{rrrrrr}A B / 2, m \text { ( } & \text { ft ) } & \text { App. Res. } A B / 2, m(\text { ft ) } & \text { App. Res. } \\ 0.91( & 3.00) & 89.50 & 4.27( & 14.00) & 96.00 \\ 1.22( & 4.00) & 99.20 & 6.10( & 20.00) & 62.00 \\ 1.83( & 6.00) & 108.00 & 9.14( & 30.00) & 31.60 \\ 2.44( & 8.00) & 103.00 & 12.19( & 40.00) & 20.80 \\ 3.05( & 10.00) & 102.00 & 18.29( & 60.00) & 13.20 \\ 4.27( & 14.00) & 76.00 & 24.38( & 80.00) & 11.60 \\ 3.05( & 10.00) & 128.00 & 30.48(100.00) & 12.40 \\ & & & 42.67(140.00) & 17.11\end{array}$

\begin{tabular}{|c|c|c|c|c|c|}
\hline DEPTH， m ( & $f t$ & RESIS. & DEPTH， m & ft & RESIS. \\
\hline 0.45 & 1.47) & 89.88 & 3.04 & 9.99) & 38.28 \\
\hline 0.66 & $2.15)$ & 160.57 & 4.47 & $14.66)$ & 20.13 \\
\hline 0.96 & $3.16)$ & 235.20 & 6.56 & $21.52)$ & 26.86 \\
\hline 1.41 & $4.64)$ & 226.96 & 9.63 & $31.58)$ & 7.30 \\
\hline 2.07 & $6.80)$ & 118.20 & $\begin{array}{r}14.13 \\
99999.00\end{array}$ & 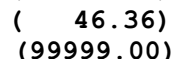 & $\begin{array}{r}4.39 \\
31.39\end{array}$ \\
\hline
\end{tabular}



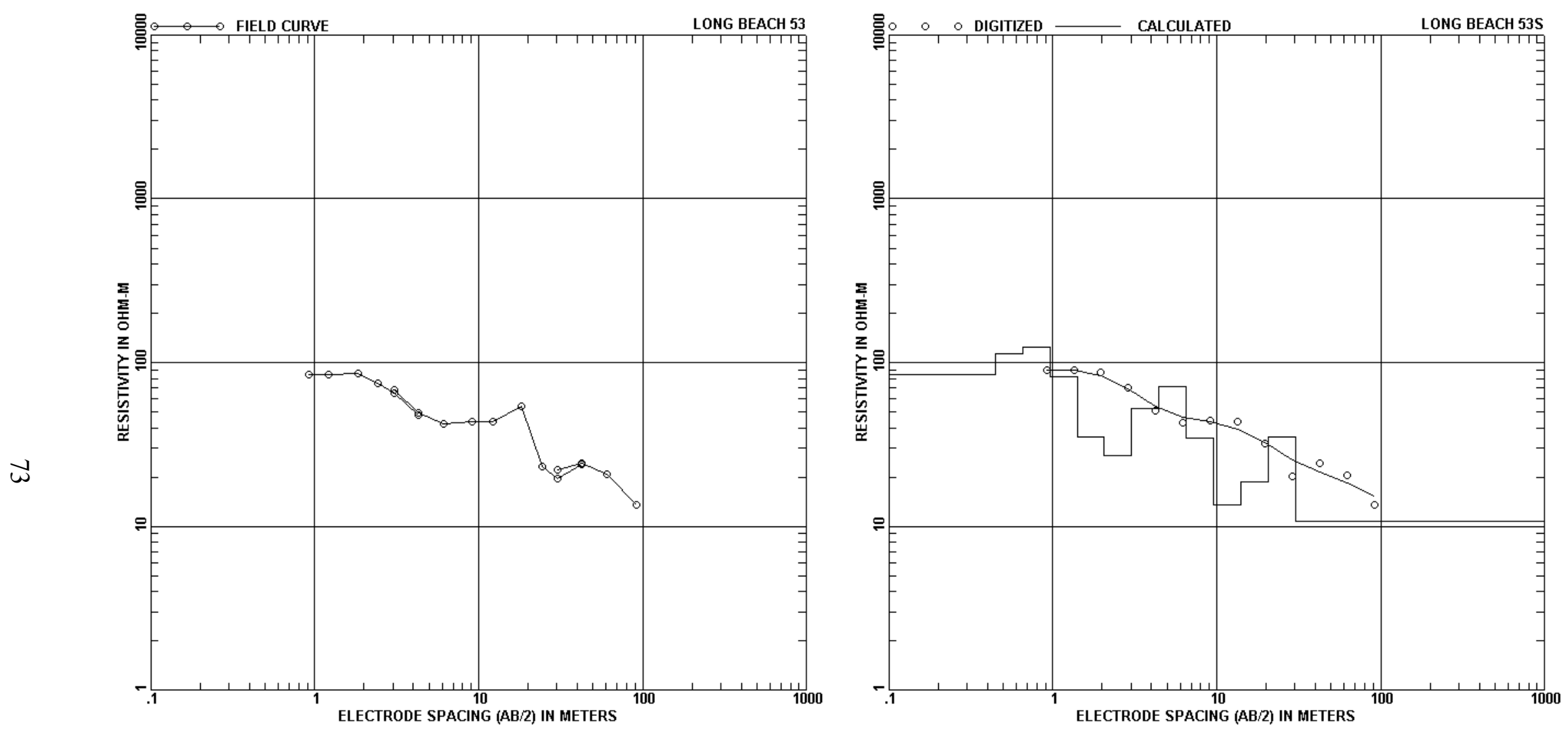

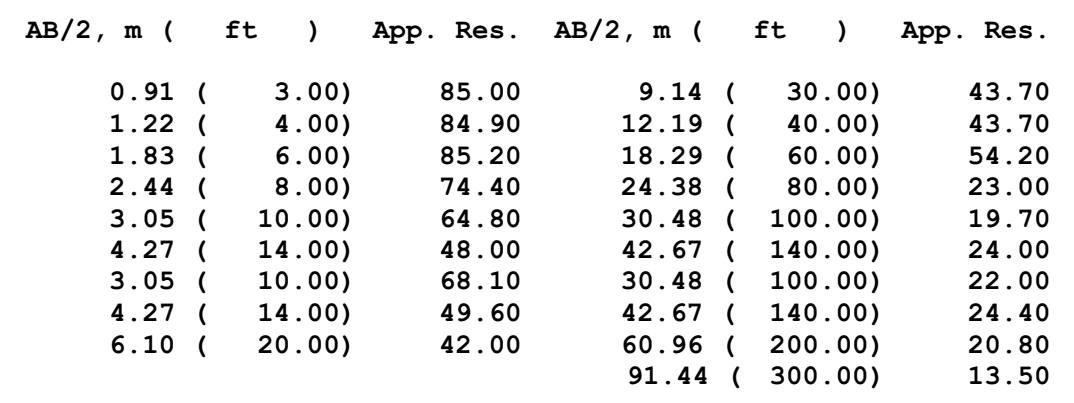

\begin{tabular}{|c|c|c|c|c|c|}
\hline DEPTH， m ( & ft & RESIS. & DEPTH， m ( & ft & RESIS. \\
\hline 0.44 & $1.46)$ & 84.43 & 4.44 & $14.58)$ & 54.91 \\
\hline 0.65 & $2.14)$ & 113.50 & 6.52 & $21.40)$ & 65.23 \\
\hline 0.96 & $3.14)$ & 124.17 & 9.57 & $31.41)$ & 28.34 \\
\hline 1.41 & $4.61)$ & 79.80 & 14.05 & $46.11)$ & 13.10 \\
\hline 2.06 & $6.77)$ & 34.95 & 20.63 & $67.67)$ & 22.12 \\
\hline \multirow[t]{2}{*}{3.03} & 9.93) & 29.03 & 30.28 & $99.33)$ & 36.26 \\
\hline & & & 99999.00 & $(99999.00)$ & 10.24 \\
\hline
\end{tabular}



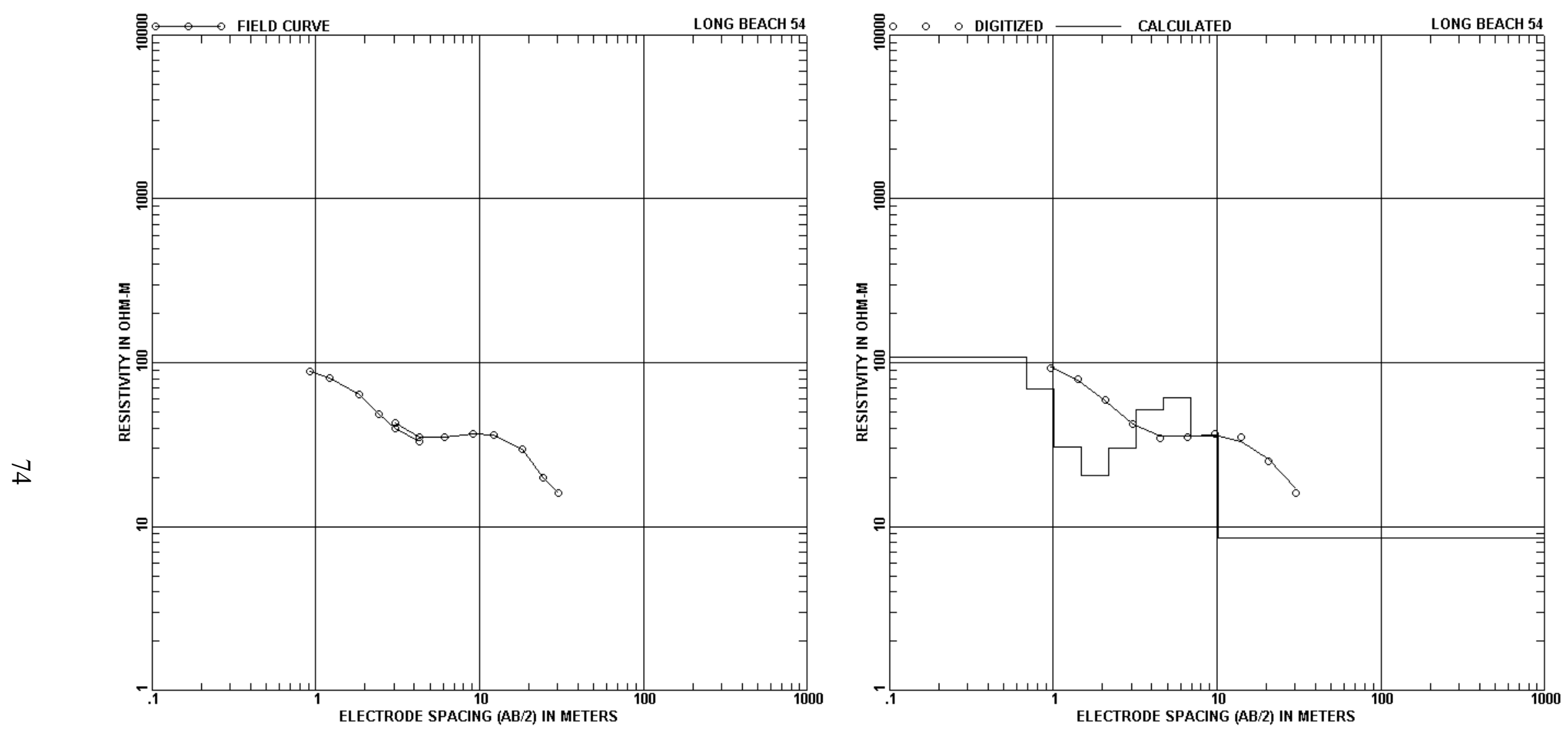

$\begin{array}{rrrrrr}A B / 2, \mathrm{~m} \text { ( } & \text { ft }) & \text { App. Res. } & A B / 2, m(\text { ft ) } & \text { App. Res. } \\ 0.91( & 3.00) & 89.10 & 4.27( & 14.00) & 35.00 \\ 1.22( & 4.00) & 81.00 & 6.10( & 20.00) & 35.00 \\ 1.83( & 6.00) & 63.60 & 9.14( & 30.00) & 36.50 \\ 2.44( & 8.00) & 48.20 & 12.19( & 40.00) & 36.00 \\ 3.05( & 10.00) & 39.90 & 18.29( & 60.00) & 29.50 \\ 4.27( & 14.00) & 33.10 & 24.38( & 80.00) & 19.90 \\ 3.05( & 10.00) & 43.10 & 30.48(100.00) & 16.10\end{array}$

\begin{tabular}{|c|c|c|c|c|c|}
\hline DEPTH， m ( & ft & RESIS. & DEPTH， m ( & $\mathrm{ft}$ & RESIS. \\
\hline 0.47 & $1.54)$ & 108.07 & 3.19 & $10.47)$ & 30.19 \\
\hline 0.69 & $2.26)$ & 108.40 & 4.68 & $15.37)$ & 51.78 \\
\hline 1.01 & $3.31)$ & 69.14 & 6.88 & $22.56)$ & 60.89 \\
\hline 1.48 & $4.86)$ & 30.65 & 10.09 & $33.11)$ & 35.53 \\
\hline $2.17 i$ & $7.13)$ & 20.48 & 99999.00 & (99999.00) & 8.48 \\
\hline
\end{tabular}



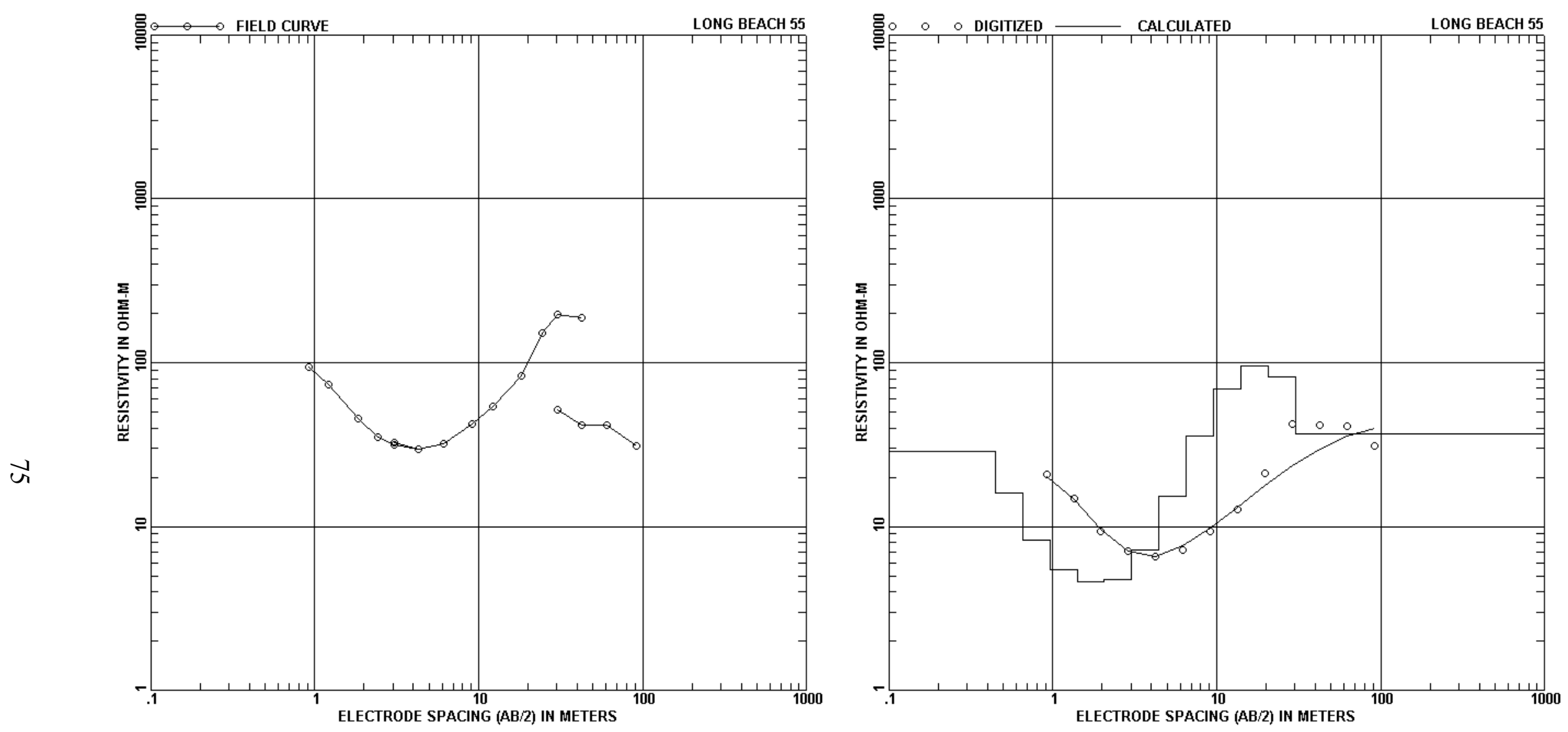

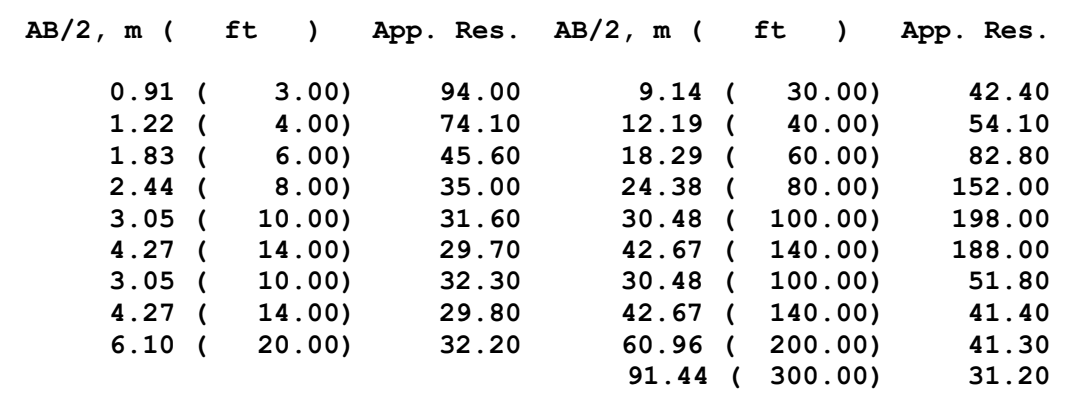

\begin{tabular}{|c|c|c|c|c|c|}
\hline DEPTH, $\mathrm{m}$ ( & ft & RESIS. & DEPTH， $\mathrm{m}$ ( & ft & RESIS. \\
\hline 0.44 & $1.46)$ & 28.76 & 4.44 & $14.58)$ & 7.17 \\
\hline 0.65 & $2.14)$ & 16.12 & 6.52 & $21.40)$ & 15.38 \\
\hline 0.96 & $3.14)$ & 8.22 & 9.57 & $31.41)$ & 35.58 \\
\hline 1.41 & $4.61)$ & 5.49 & 14.05 & $46.11)$ & 68.76 \\
\hline 2.06 & $6.77)$ & 4.63 & 20.63 & $67.67)$ & 95.40 \\
\hline 3.03 & $9.93)$ & 4.73 & 30.28 & $99.33)$ & 82.34 \\
\hline & & & 99999.00 & $(99999.00)$ & 37.01 \\
\hline
\end{tabular}



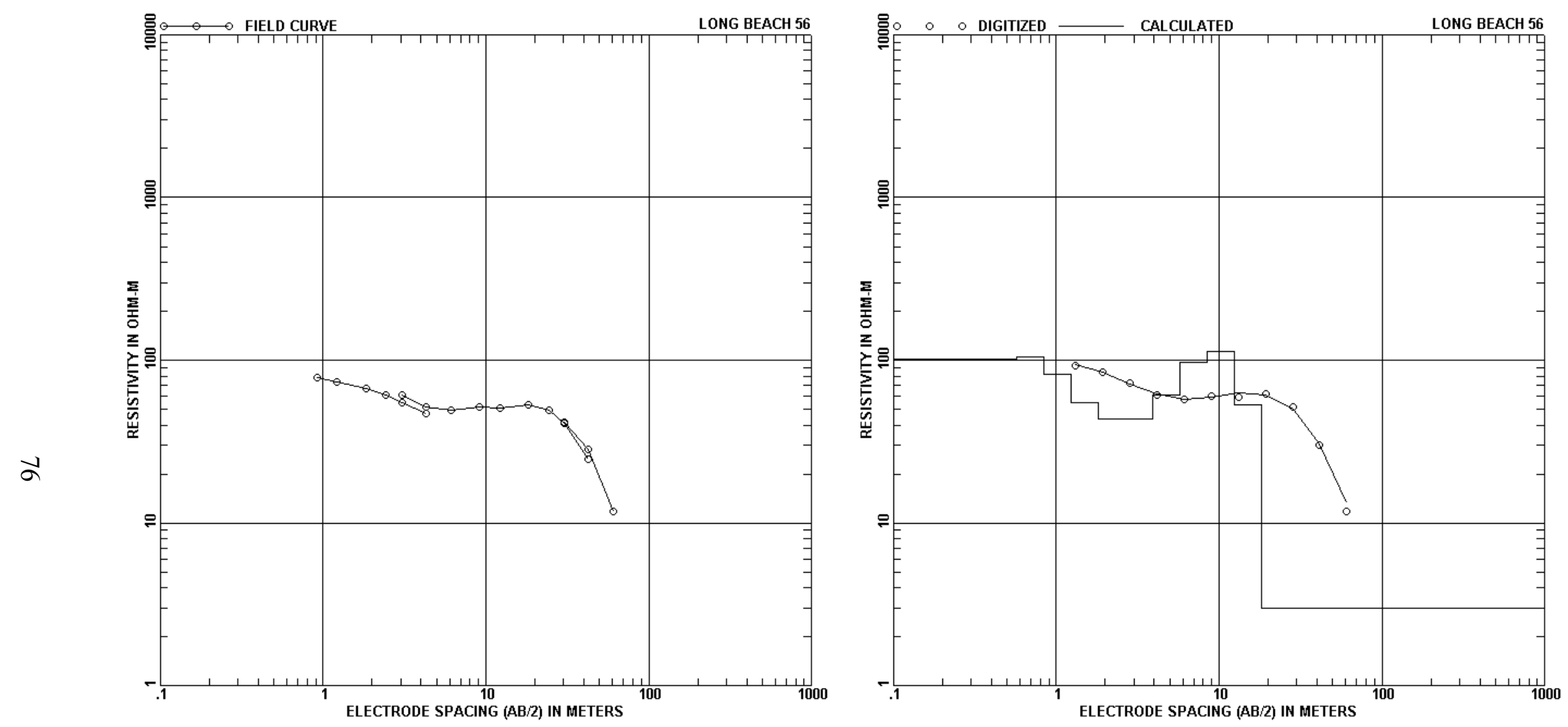

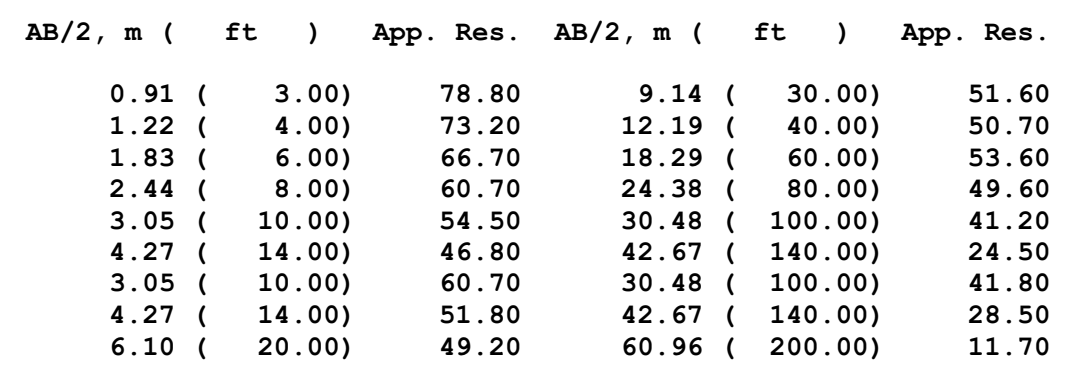

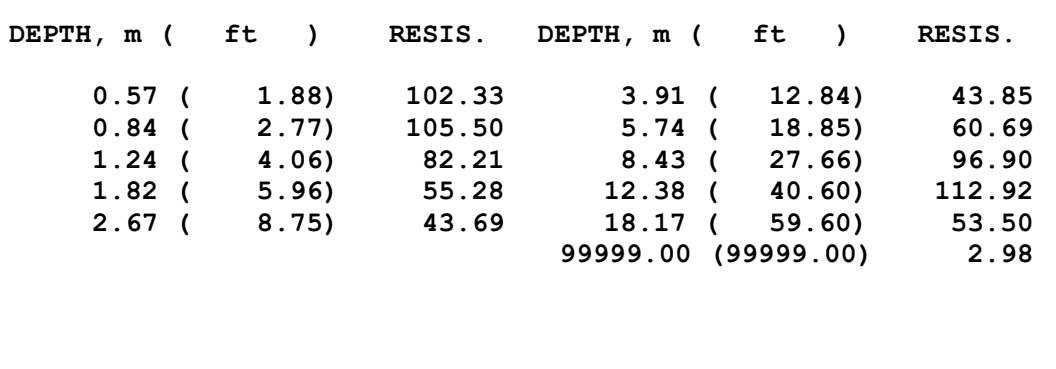



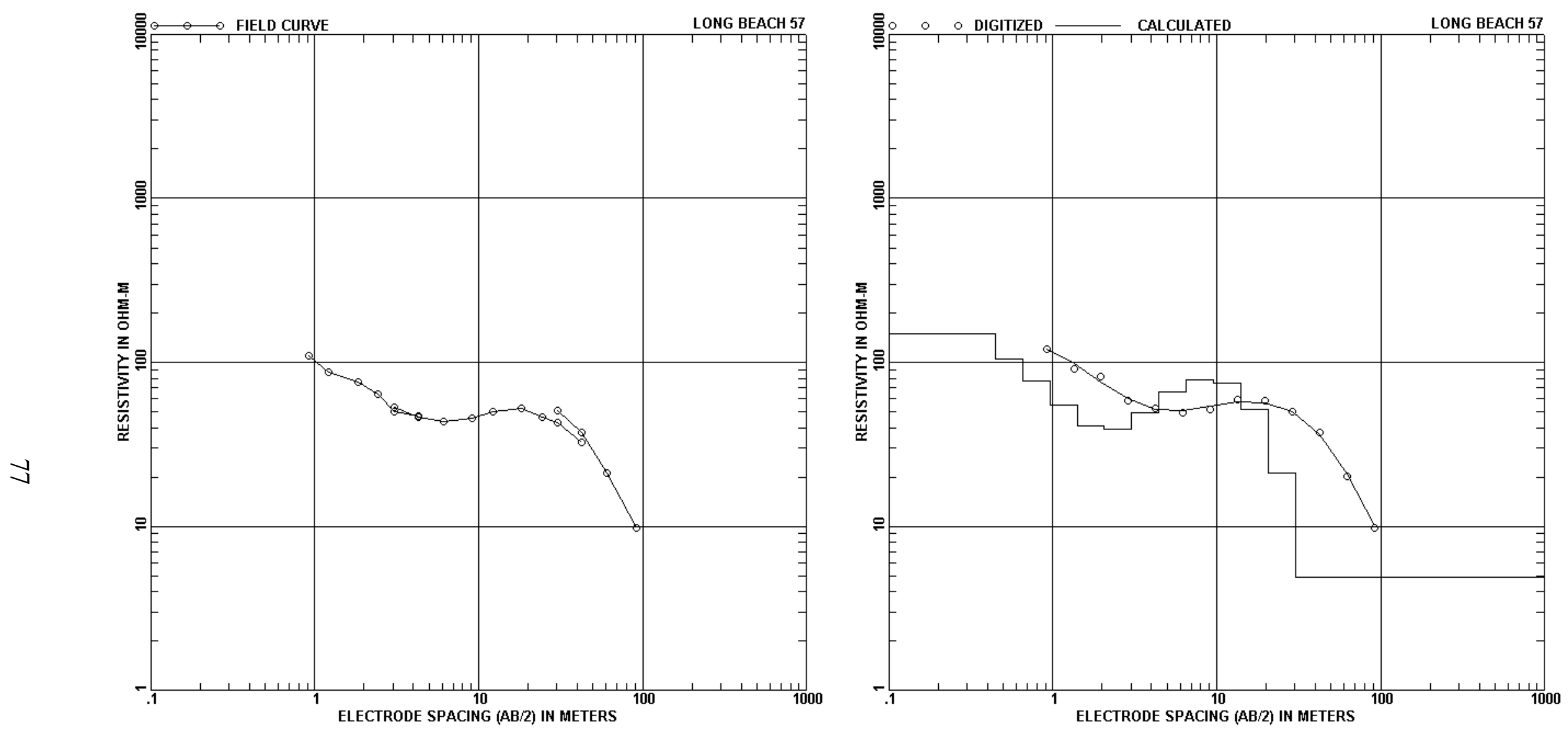

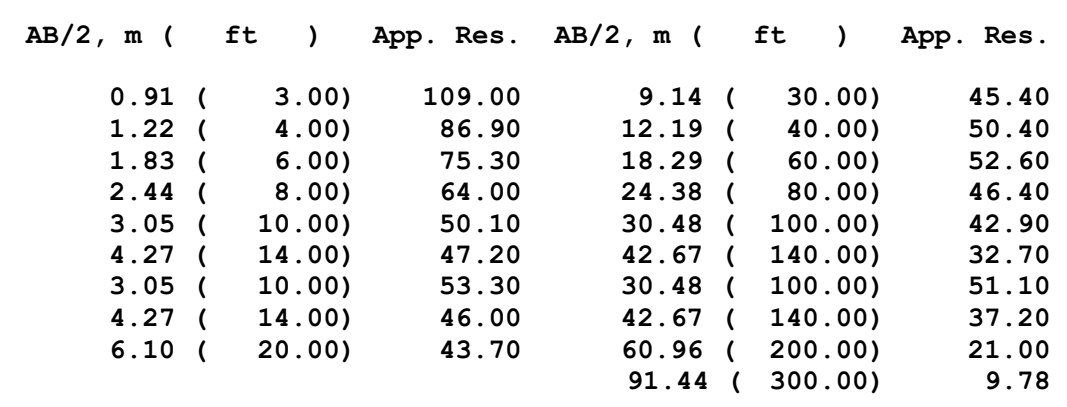

\begin{tabular}{|c|c|c|c|c|c|}
\hline DEPTH， m ( & ft , & RESIS. & DEPTH， m & $1 \mathrm{ft}$ & RESIS. \\
\hline 0.44 & $1.46)$ & 149.04 & 4.44 & $14.58)$ & 49.22 \\
\hline 0.65 & $2.14)$ & 105.04 & 6.52 & $21.40)$ & 66.43 \\
\hline 0.96 & $3.14)$ & 76.56 & 9.57 & $31.41)$ & 78.61 \\
\hline 1.41 & $4.61)$ & 54.51 & 14.05 & $46.11)$ & 75.02 \\
\hline 2.06 & $6.77)$ & 40.67 & 20.63 & $67.67)$ & 51.74 \\
\hline 3.03 & $9.93)$ & 38.83 & 30.28 & $99.33)$ & 21.20 \\
\hline & & & 99999.00 & $(99999.00)$ & 4.93 \\
\hline
\end{tabular}



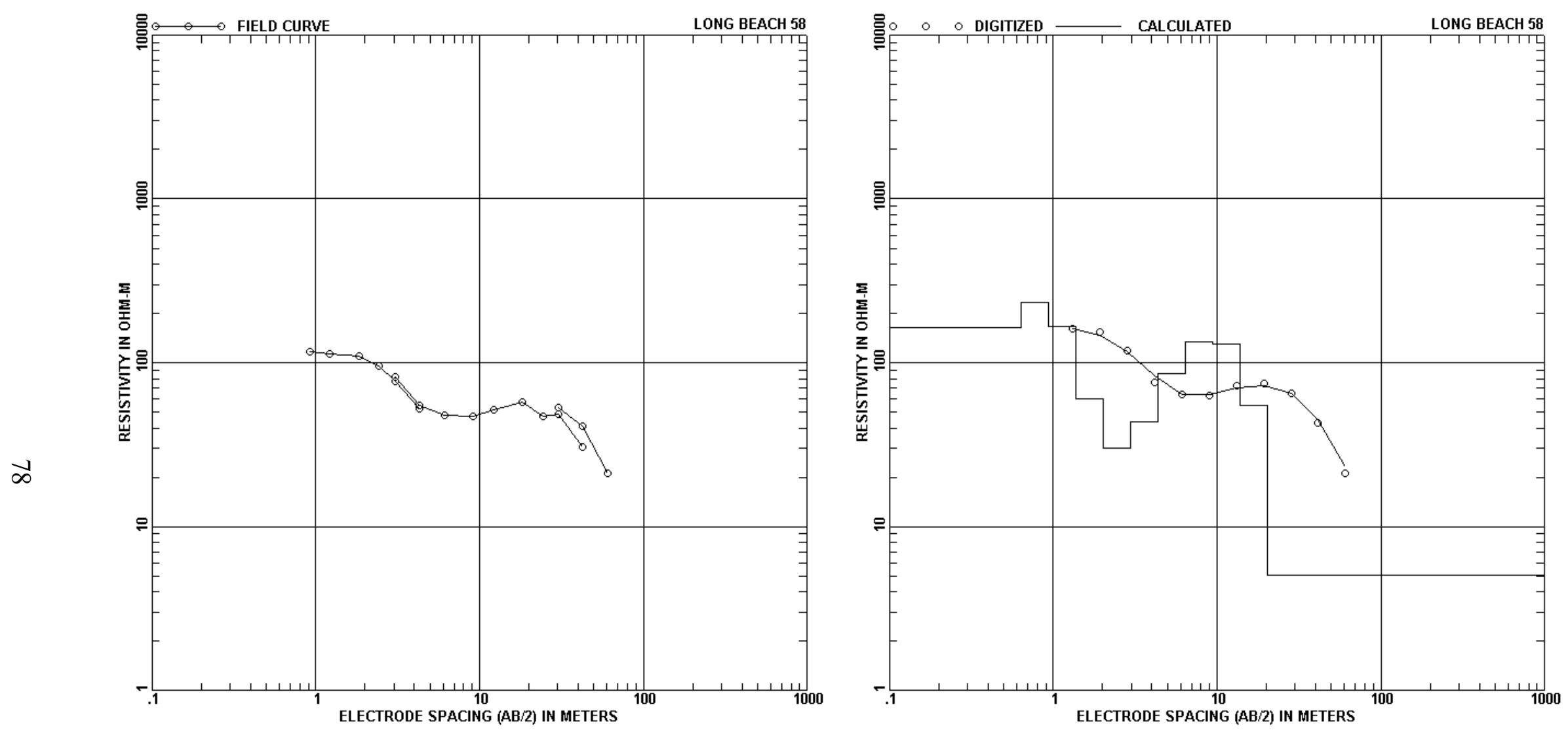

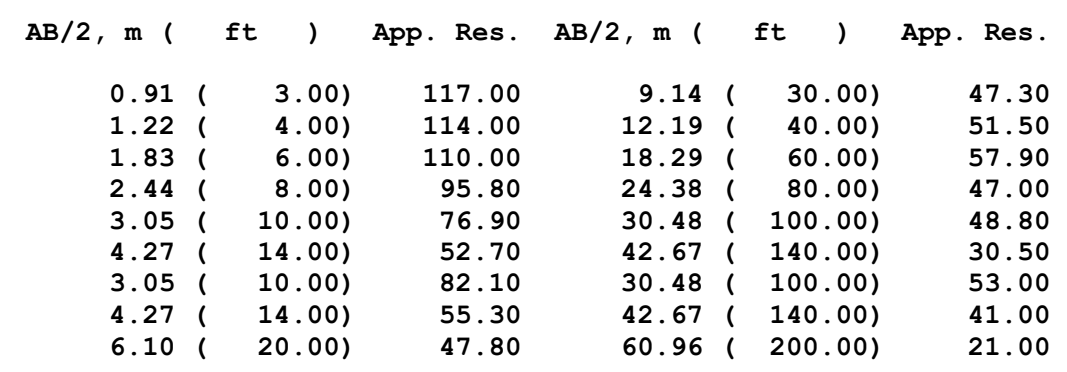

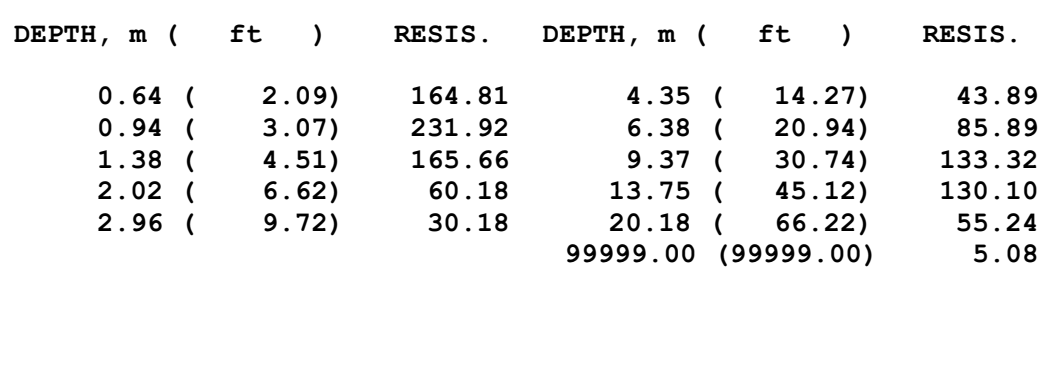



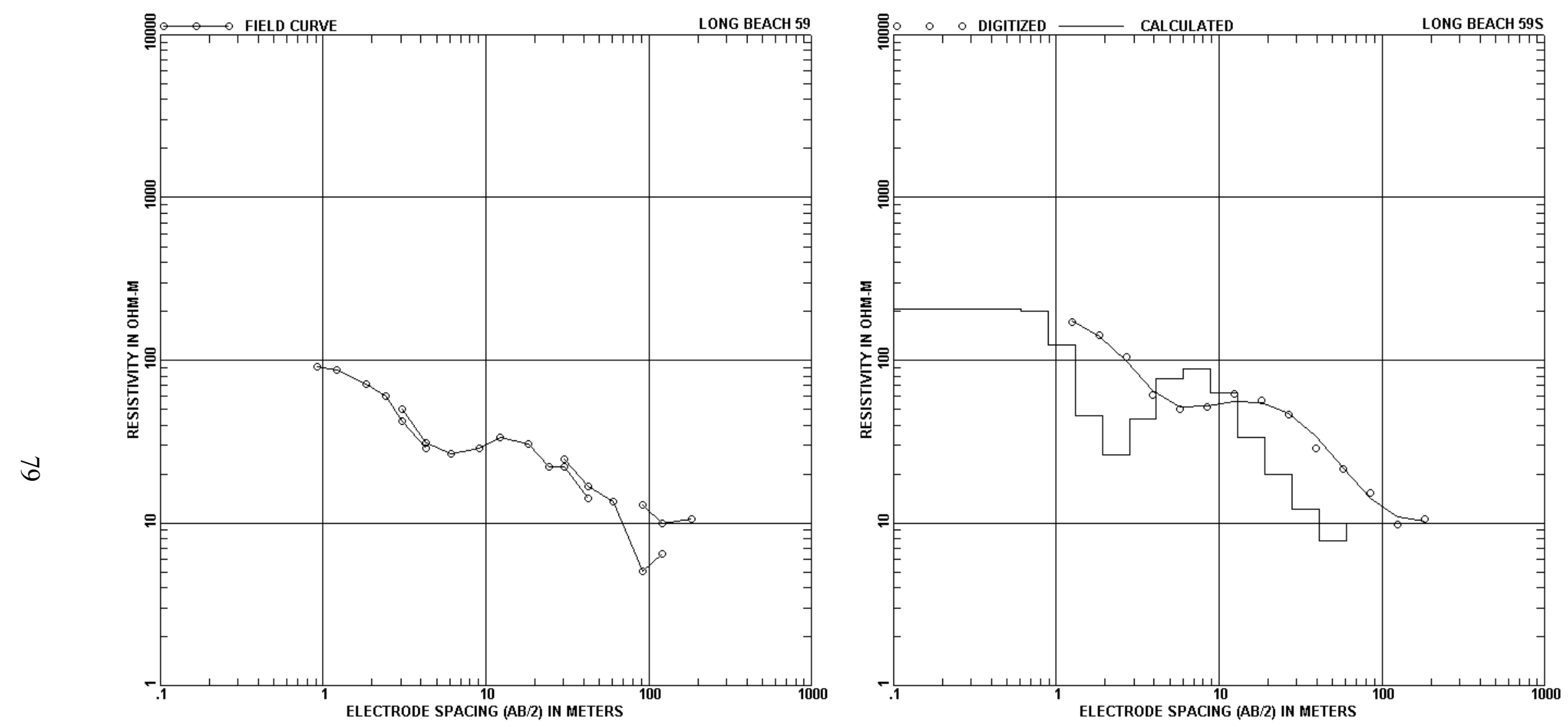

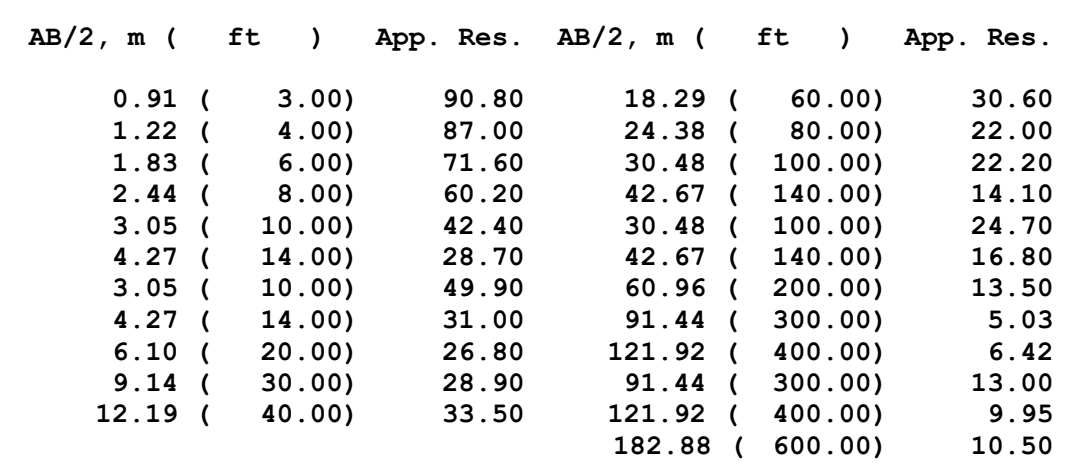

\begin{tabular}{|c|c|c|c|c|c|}
\hline DEPTH， m ( & ft ) & RESIS. & DEPTH， $\mathrm{m}$ ( & ft & RESIS. \\
\hline 0.611 & 1.99) & 207.55 & 8.89 & $29.16)$ & 88.31 \\
\hline $0.89 i$ & $2.92)$ & 199.61 & 13.05 & $42.80)$ & 63.16 \\
\hline $1.30 i$ & $4.28)$ & 124.97 & 19.15 & $62.82)$ & 33.71 \\
\hline 1.91 i & $6.28)$ & 45.48 & 28.11 & $92.21)$ & 19.72 \\
\hline $2.81 i$ & $9.22)$ & 26.19 & 41.25 & $135.35)$ & 12.22 \\
\hline $4.13 i$ & $13.53)$ & 43.58 & 60.55 & ( 198.66$)$ & 7.72 \\
\hline $6.06 i$ & 19.87) & 76.93 & 99999.00 & $(99999.00)$ & 9.95 \\
\hline
\end{tabular}



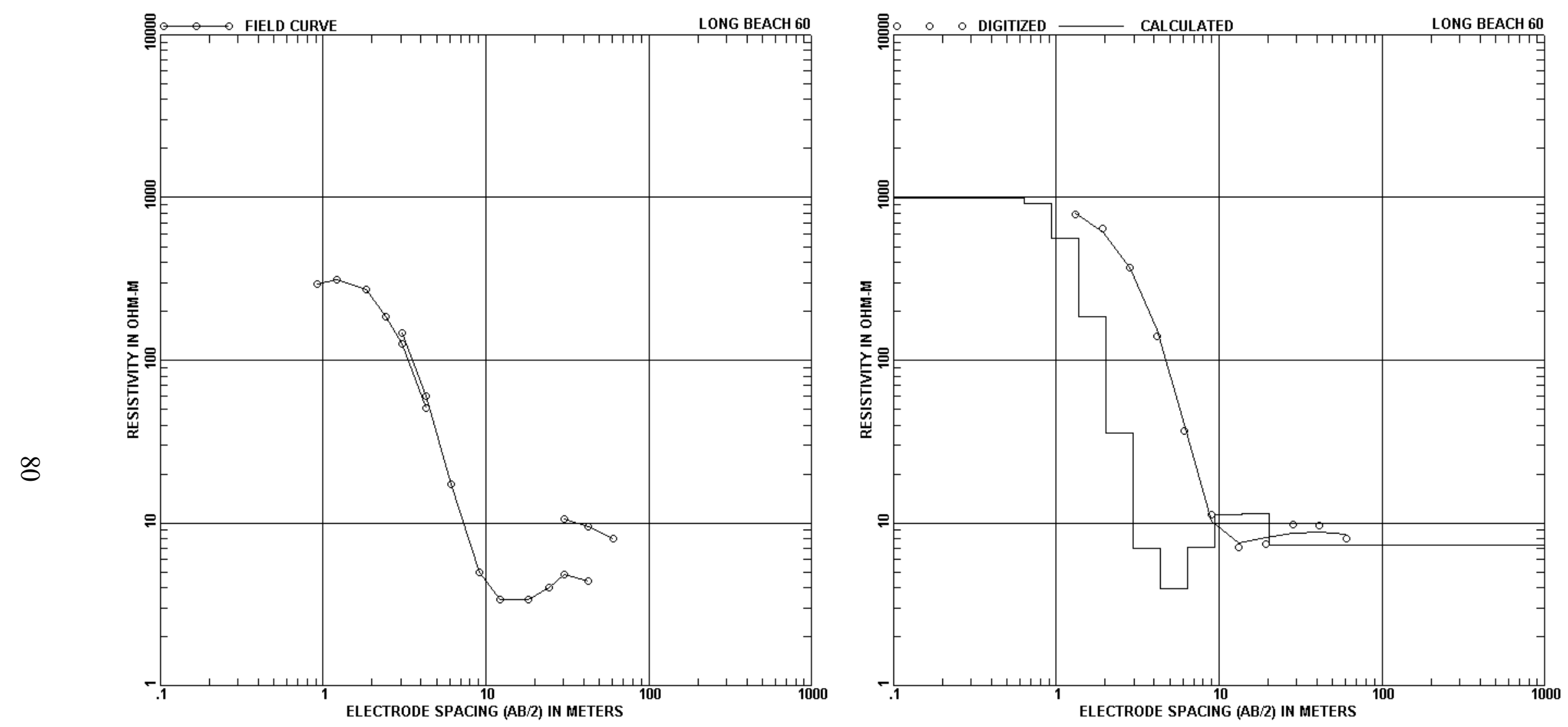

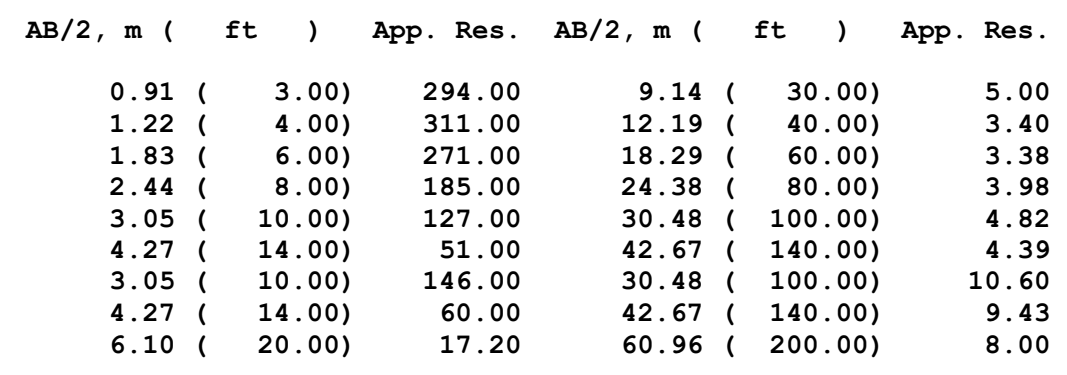

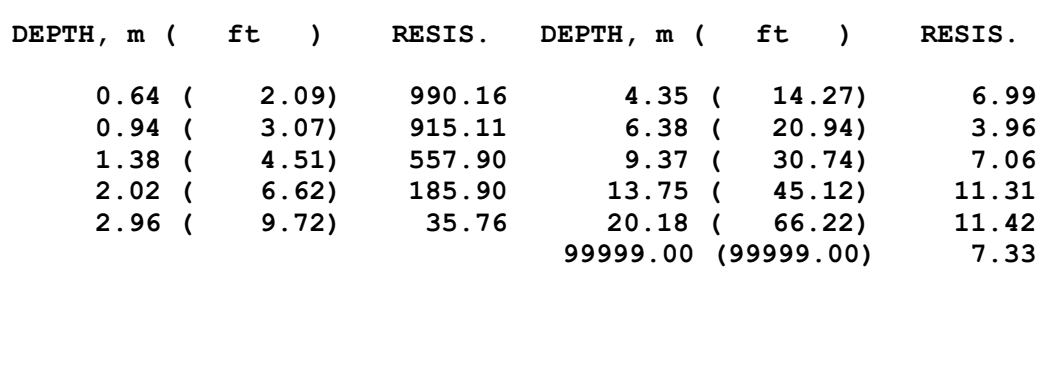



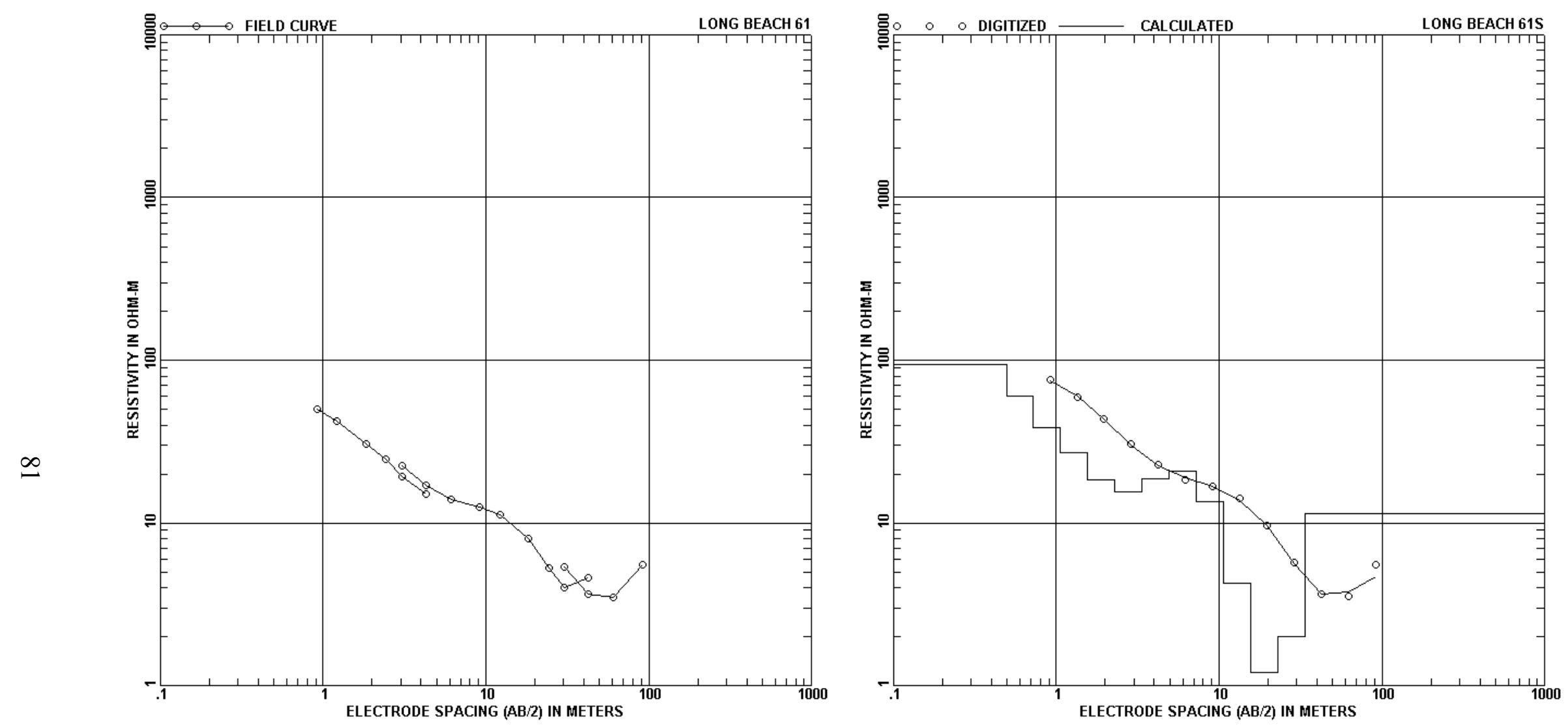

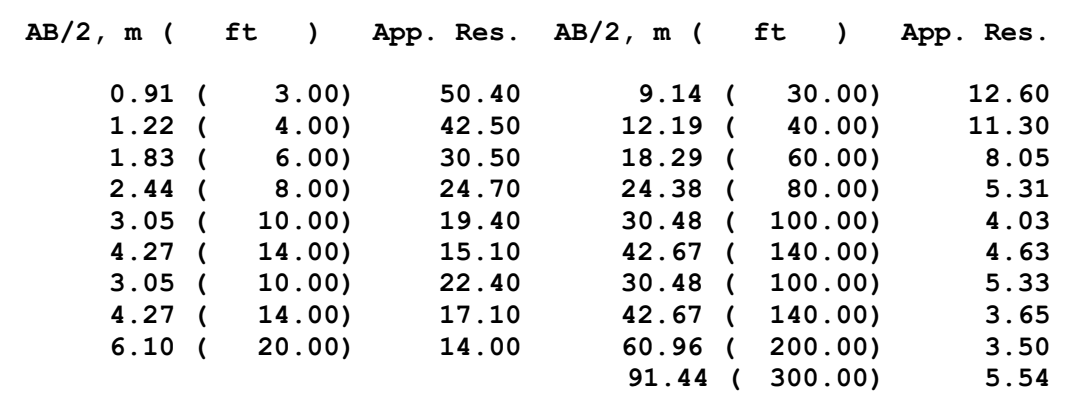

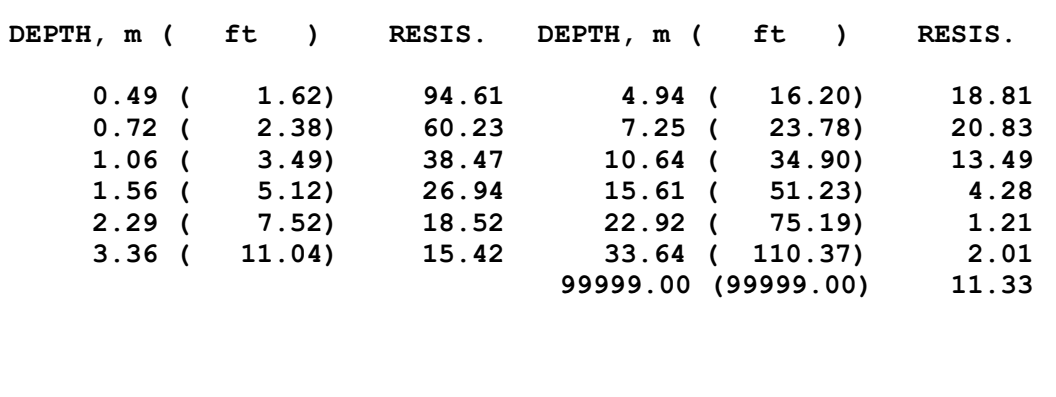



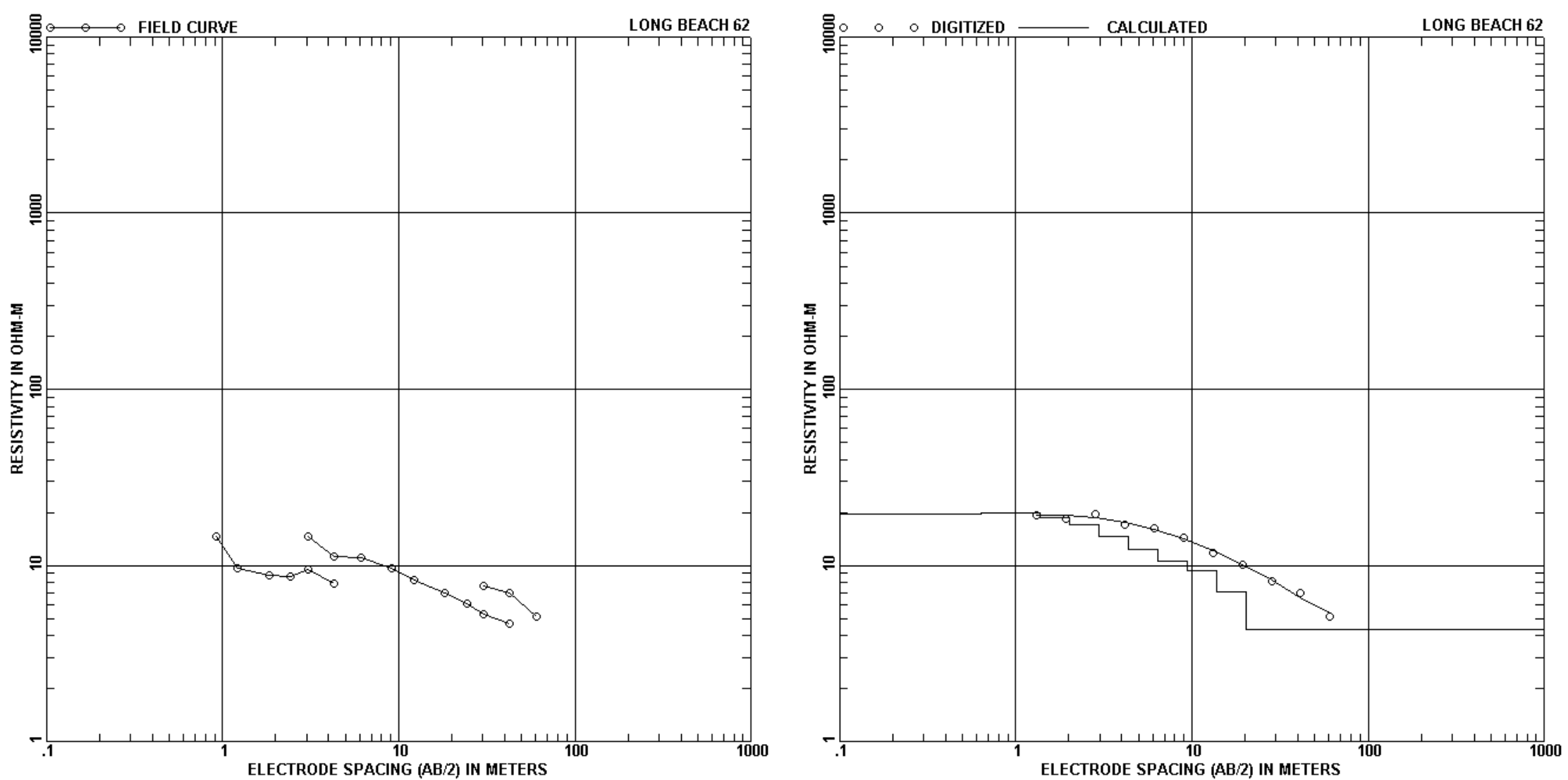

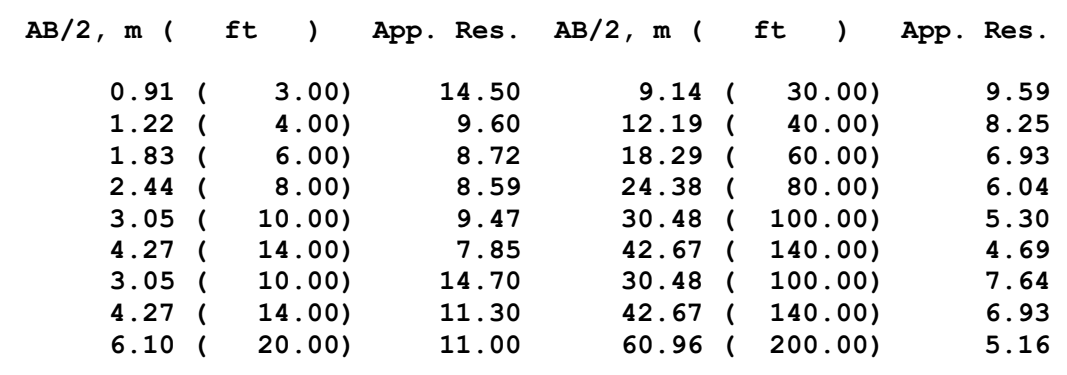

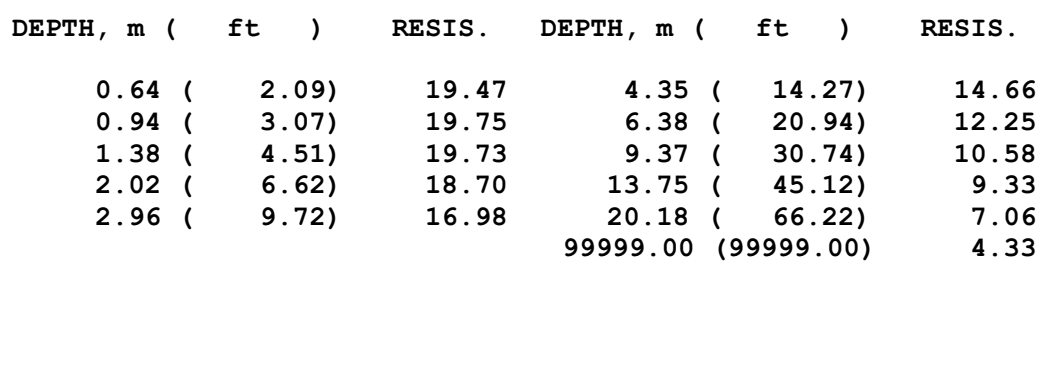



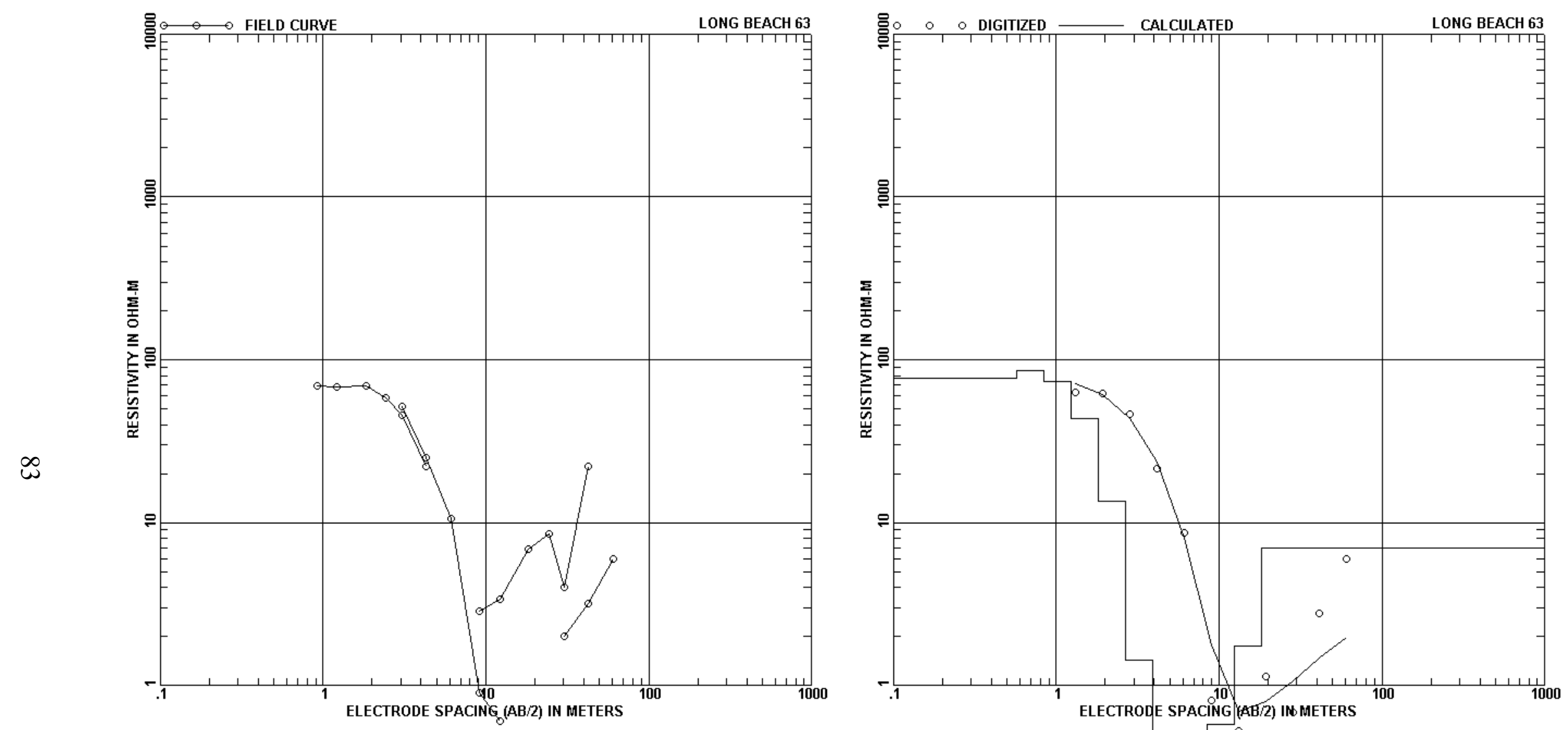

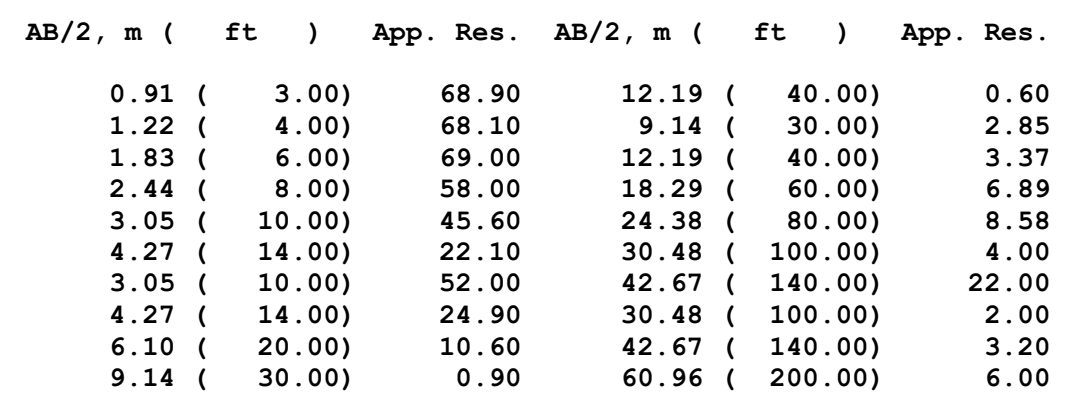

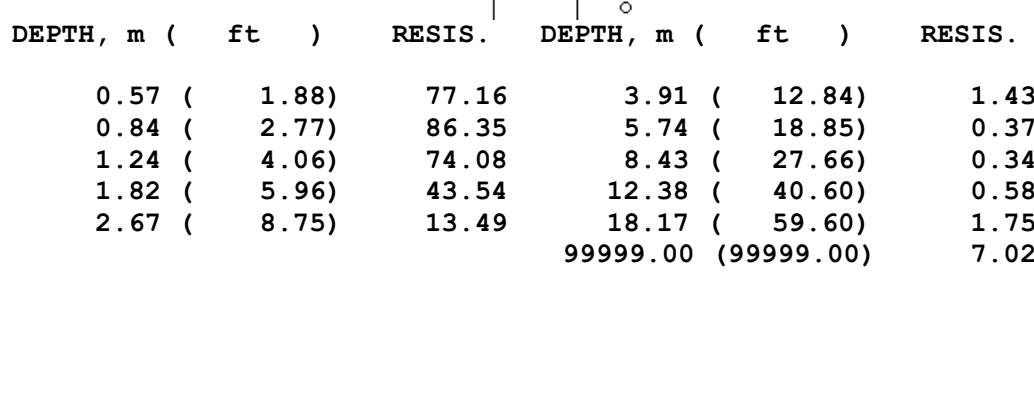



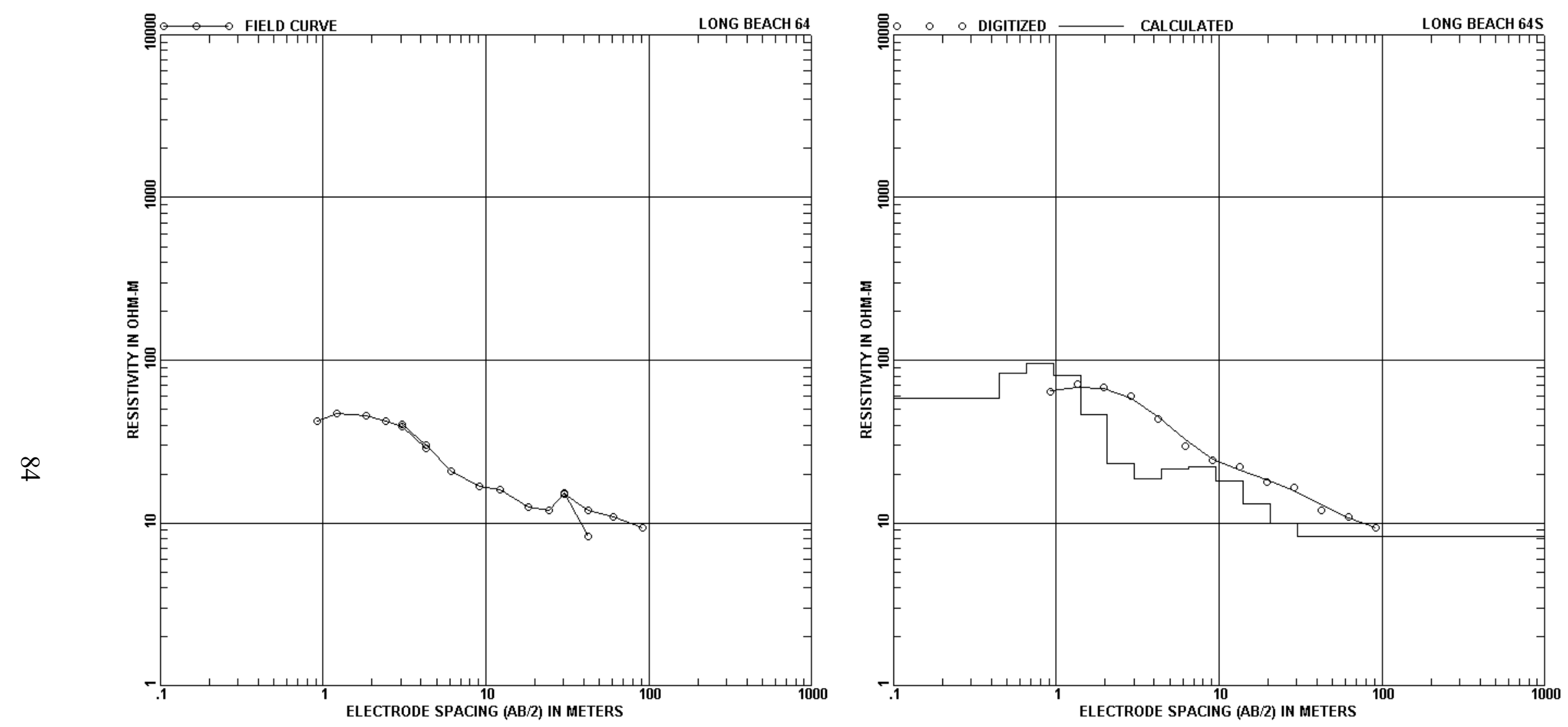

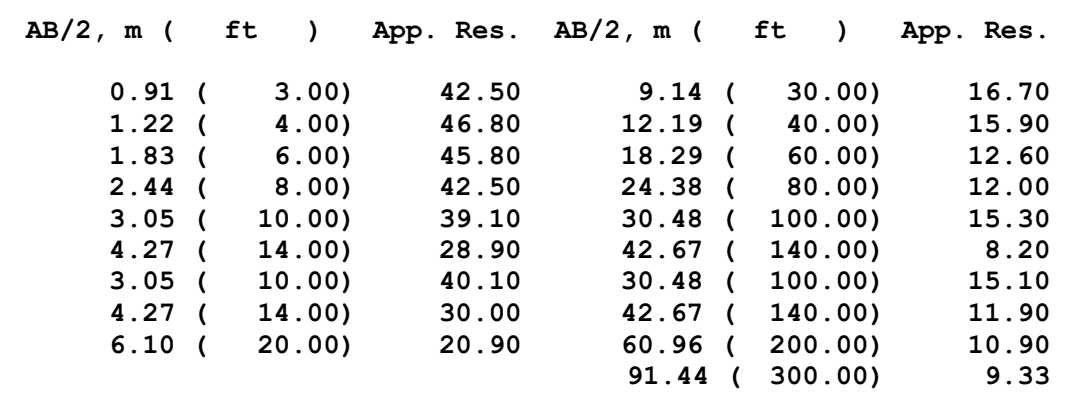

\begin{tabular}{|c|c|c|c|c|c|}
\hline DEPTH, $\mathrm{m}$ ( & ft & RESIS. & DEPTH， $\mathrm{m}$ ( & ft & RESIS. \\
\hline 0.44 & $1.46)$ & 58.05 & 4.44 & $14.58)$ & 18.56 \\
\hline 0.65 & $2.14)$ & 82.55 & 6.52 & $21.40)$ & 21.30 \\
\hline 0.96 & $3.14)$ & 95.95 & 9.57 & $31.41)$ & 21.96 \\
\hline 1.41 & $4.61)$ & 80.68 & 14.05 & $46.11)$ & 18.13 \\
\hline 2.06 & $6.77)$ & 46.19 & 20.63 & $67.67)$ & 13.06 \\
\hline 3.03 & $9.93)$ & 23.34 & 30.28 & $99.33)$ & 9.98 \\
\hline & & & 99999.00 & $(99999.00)$ & 8.22 \\
\hline
\end{tabular}



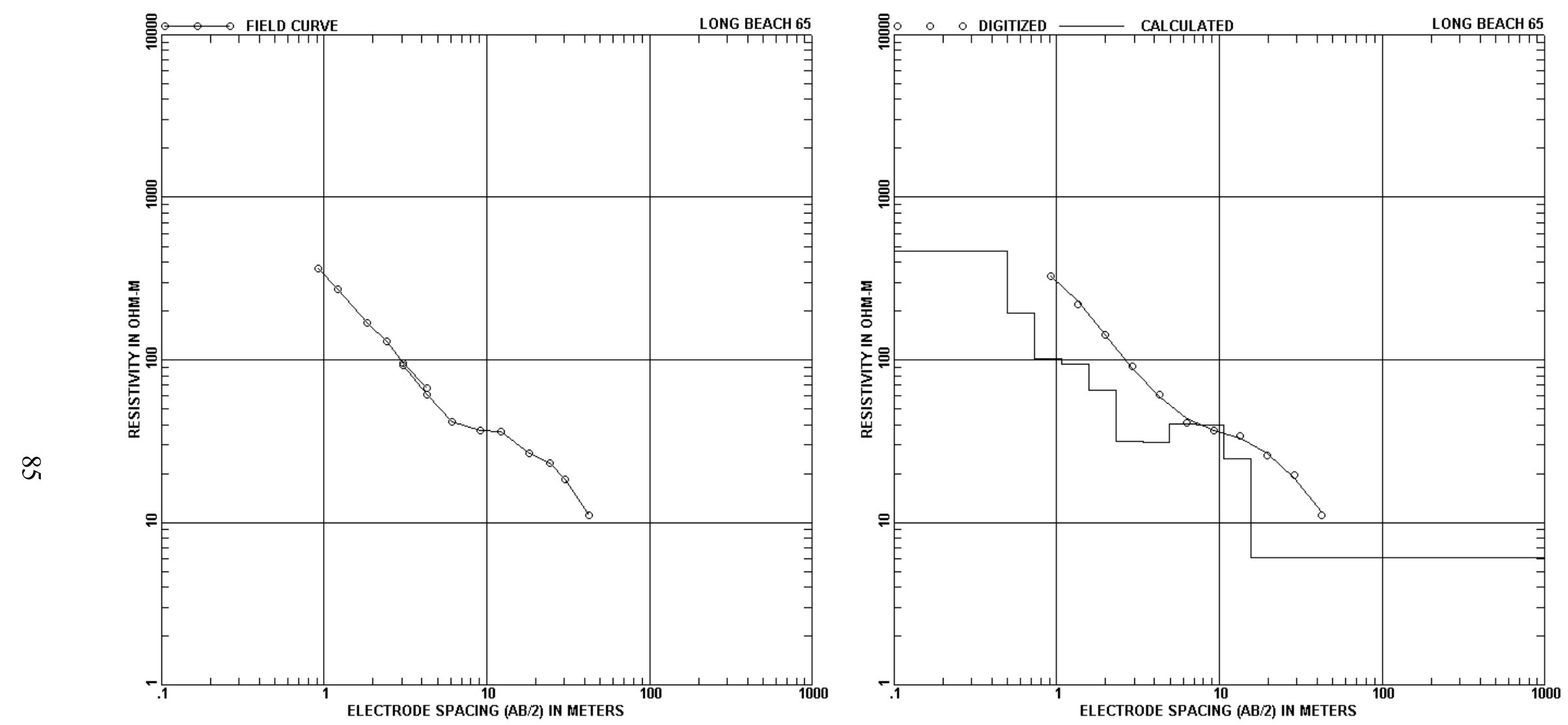

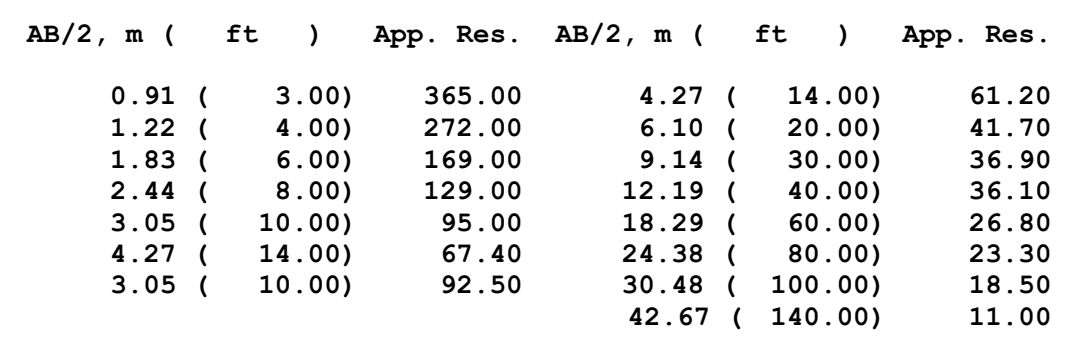

\begin{tabular}{|c|c|c|c|c|c|}
\hline DEPTH， m ( & ft & RESIS. & DEPTH， $\mathrm{m}$ ( & ft & RESIS. \\
\hline 0.501 & $1.63)$ & 467.67 & 3.38 & $11.10)$ & 31.73 \\
\hline $0.73 i$ & $2.39)$ & 192.70 & 4.96 & $16.29)$ & 31.12 \\
\hline $1.07 i$ & $3.51)$ & 101.34 & 7.29 & $23.91)$ & 40.42 \\
\hline $1.57 \%$ & $5.15)$ & 93.51 & 10.70 & $35.09)$ & 39.66 \\
\hline \multirow[t]{2}{*}{2.30} & $7.56)$ & 65.26 & 15.70 & $51.51)$ & 24.51 \\
\hline & & & 99999.00 & $(99999.00)$ & 6.03 \\
\hline
\end{tabular}



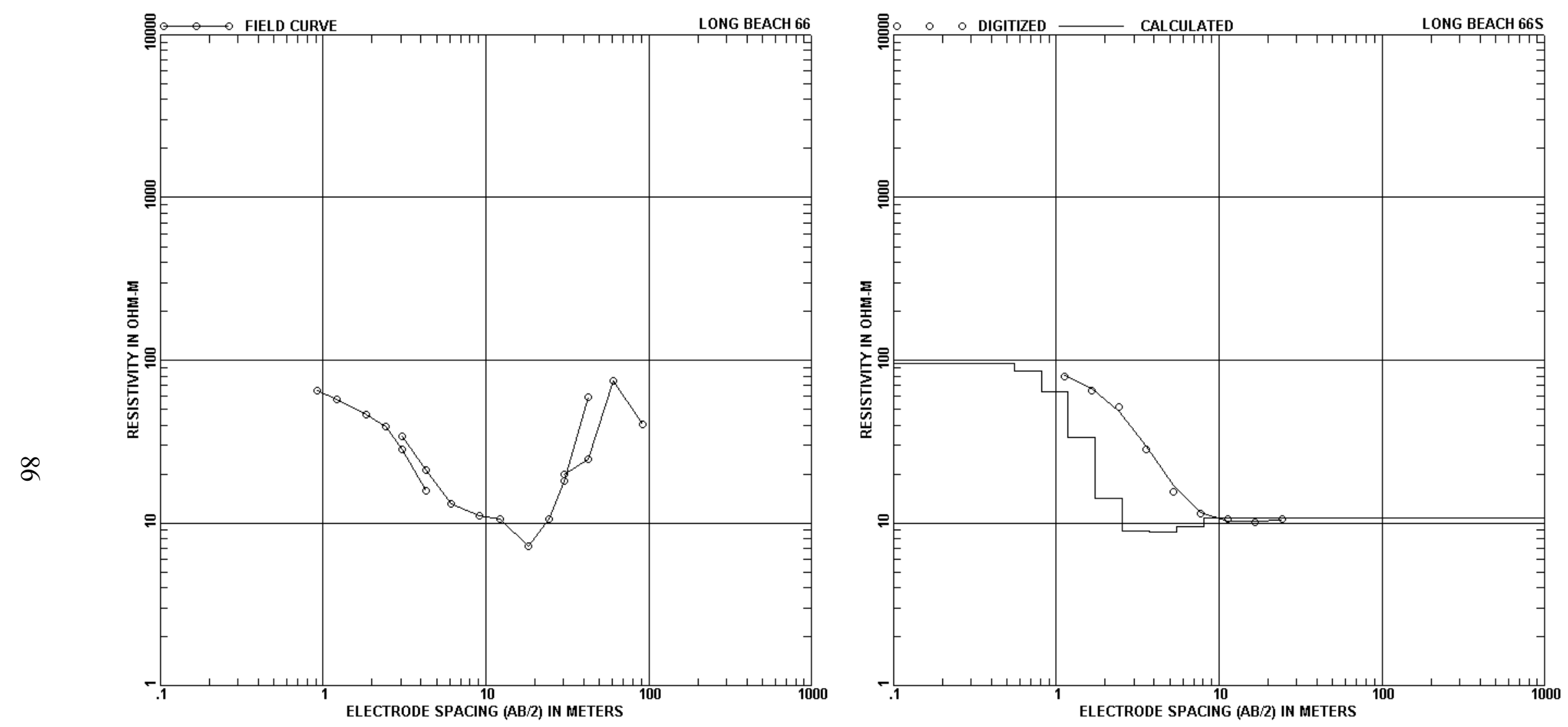

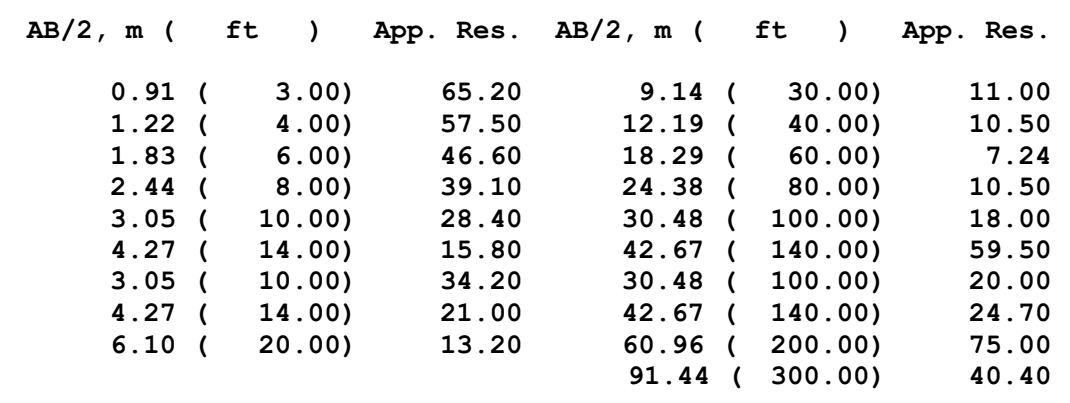

\begin{tabular}{|c|c|c|c|c|c|}
\hline DEPTH， m ( & ) & RESIS. & DEPTH， m ( & ft & RESIS. \\
\hline 0.55 ( & $1.80)$ & 94.98 & 2.55 & $8.38)$ & 14.12 \\
\hline $0.81 i$ & $2.65)$ & 85.25 & 3.75 & $12.29)$ & 8.99 \\
\hline 1.19 & $3.89)$ & 63.92 & 5.50 & $18.05)$ & 8.85 \\
\hline 1.74 ( & $5.71)$ & 33.55 & $\begin{array}{r}8.07 \\
99999.00\end{array}$ & $\begin{array}{l}(26.49) \\
(99999.00)\end{array}$ & $\begin{array}{r}9.54 \\
10.67\end{array}$ \\
\hline
\end{tabular}



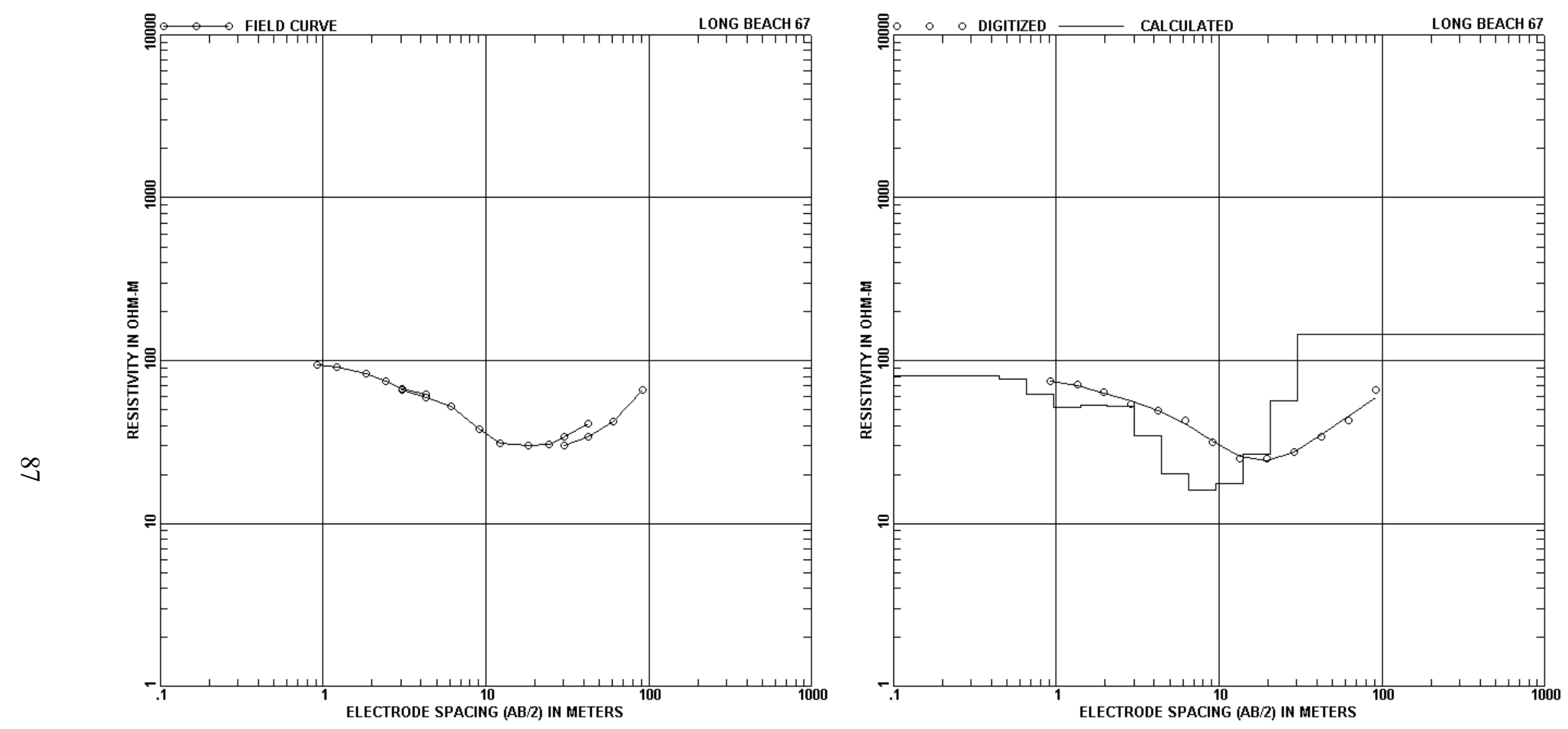

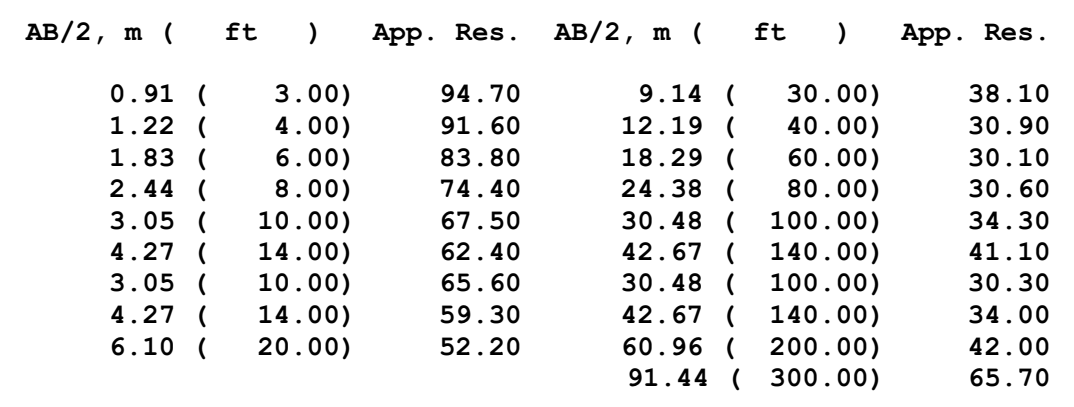

\begin{tabular}{|c|c|c|c|c|c|}
\hline DEPTH， m ( & ft & RESIS. & DEPTH， m ( & ft & RESIS. \\
\hline 0.44 & $1.46)$ & 80.14 & 4.44 & $14.58)$ & 34.78 \\
\hline 0.65 & $2.14)$ & 77.28 & 6.52 & $21.40)$ & 20.19 \\
\hline 0.96 & $3.14)$ & 62.43 & 9.57 & $31.41)$ & 16.01 \\
\hline 1.41 & $4.61)$ & 51.68 & 14.05 & $46.11)$ & 17.64 \\
\hline 2.06 & $6.77)$ & 53.35 & 20.63 & $67.67)$ & 26.67 \\
\hline \multirow[t]{2}{*}{3.03} & $9.93)$ & 52.09 & 30.28 & $99.33)$ & 56.28 \\
\hline & & & 99999.00 & $(99999.00)$ & 145.13 \\
\hline
\end{tabular}



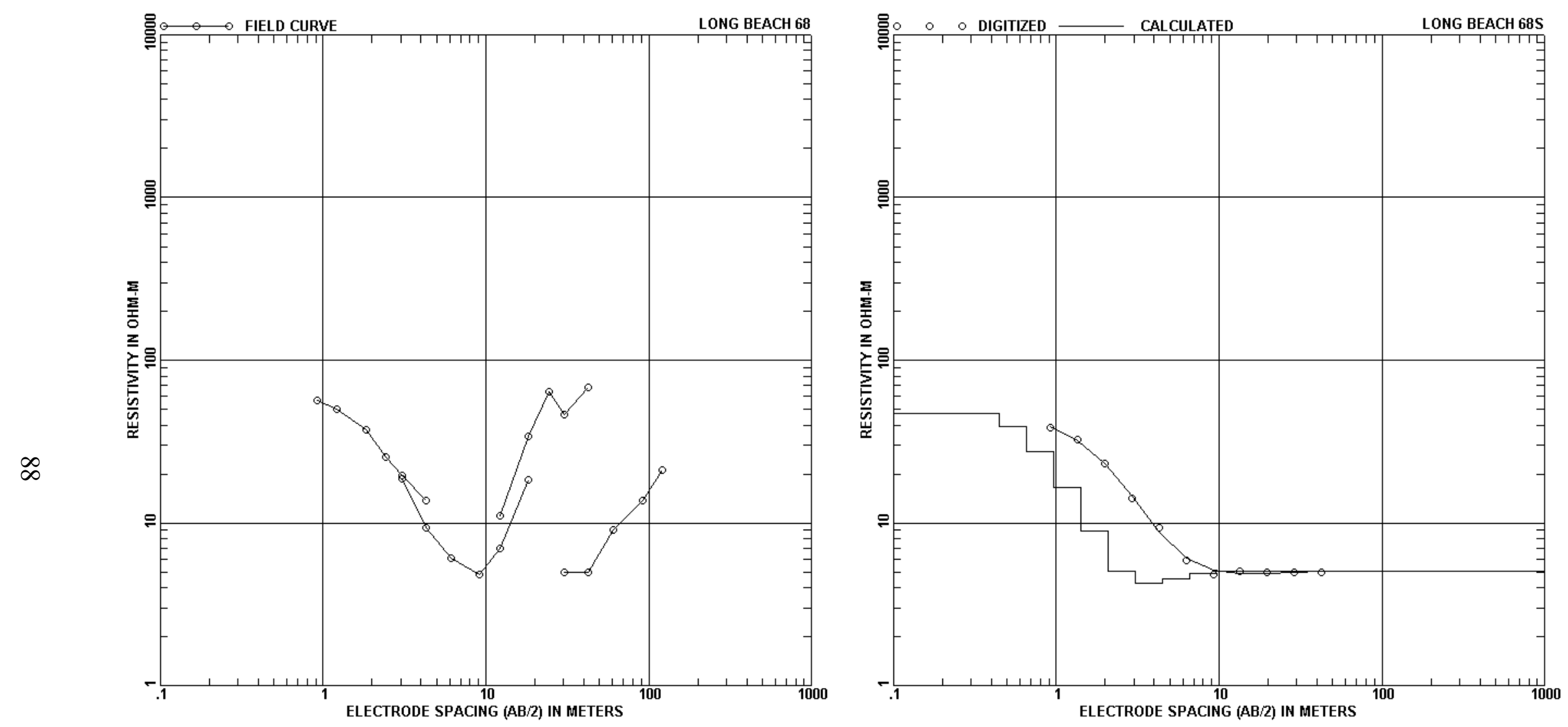

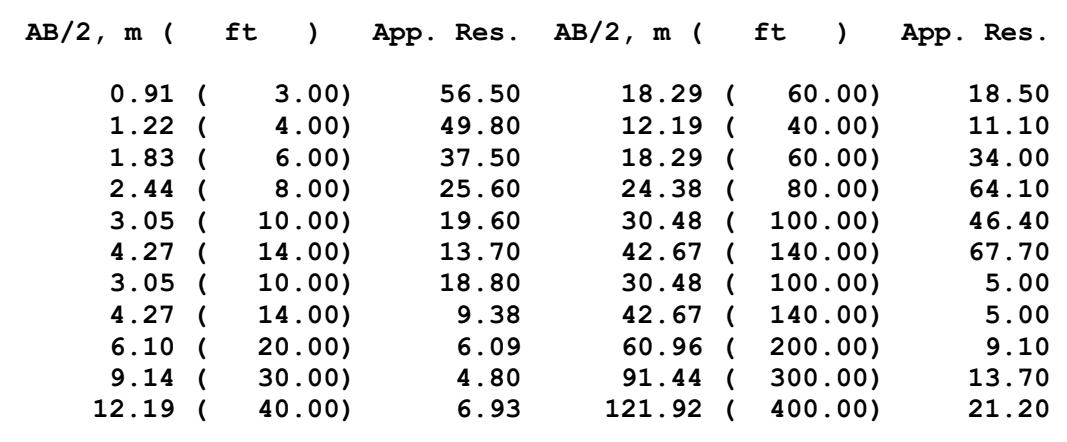

\begin{tabular}{|c|c|c|c|c|c|}
\hline DEPTH， m ( & ft ) & RESIS. & DEPTH， m ( & ft & RESIS. \\
\hline 0.45( & 1.47) & 46.89 & 3.04 & 9.99) & 5.08 \\
\hline 0.661 & 2.15) & 39.07 & 4.47 & $14.66)$ & 4.23 \\
\hline 0.961 & $3.16)$ & 27.49 & 6.56 & $21.52)$ & 4.56 \\
\hline $1.41 （$ & $4.64)$ & 16.52 & 9.63 & $31.58)$ & 4.89 \\
\hline $2.07 i$ & $6.80)$ & 8.91 & 14.13 & $46.36)$ & 5.03 \\
\hline & & & 99999.00 & $(99999.00)$ & 5.06 \\
\hline
\end{tabular}



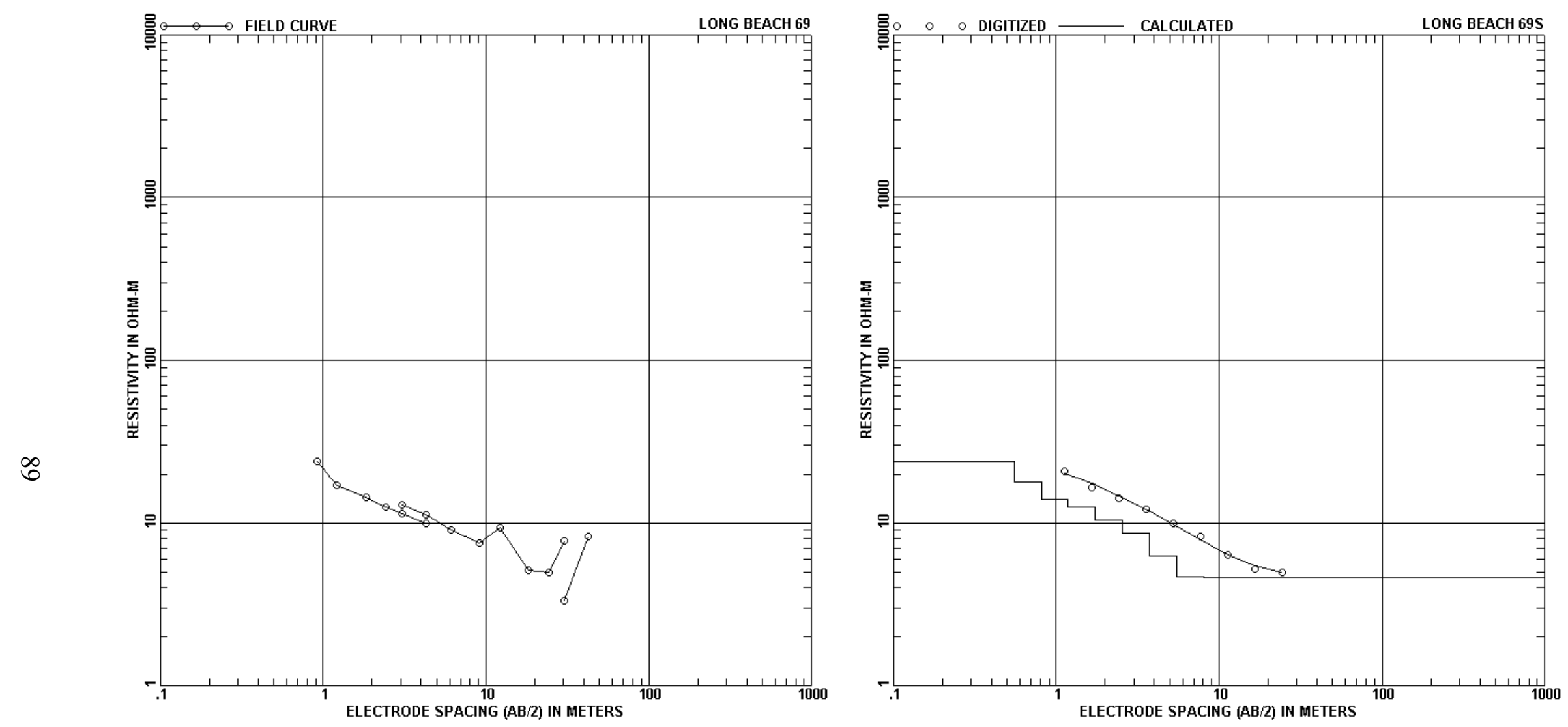

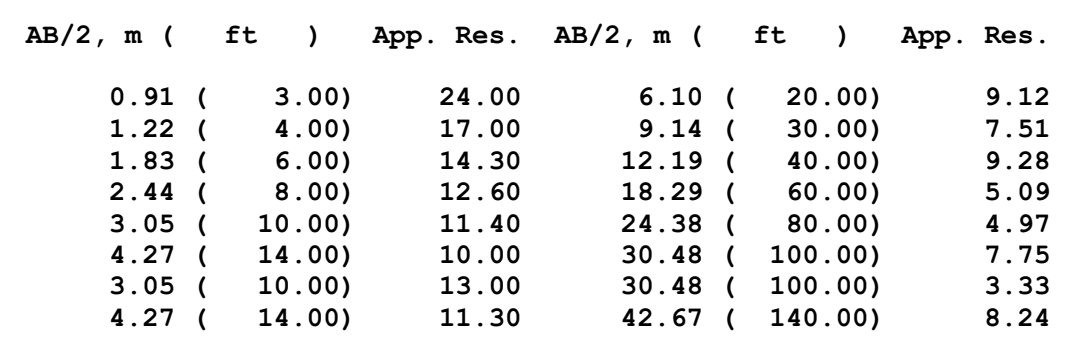

\begin{tabular}{|c|c|c|c|c|c|}
\hline DEPTH， m ( & ft , & RESIS. & DEPTH， m ( & ft & RESIS. \\
\hline 0.551 & $1.80)$ & 23.72 & 2.55 & $8.38)$ & 10.45 \\
\hline 0.811 & $2.65)$ & 17.81 & 3.75 & $12.29)$ & 8.67 \\
\hline 1.19 ( & $3.89)$ & 14.05 & 5.50 & $18.05)$ & 6.23 \\
\hline $1.74 \quad($ & $5.71)$ & 12.54 & $\begin{array}{r}8.07 \\
99999.00\end{array}$ & $\begin{array}{l}(26.49) \\
(99999.00)\end{array}$ & $\begin{array}{l}4.69 \\
4.60\end{array}$ \\
\hline
\end{tabular}



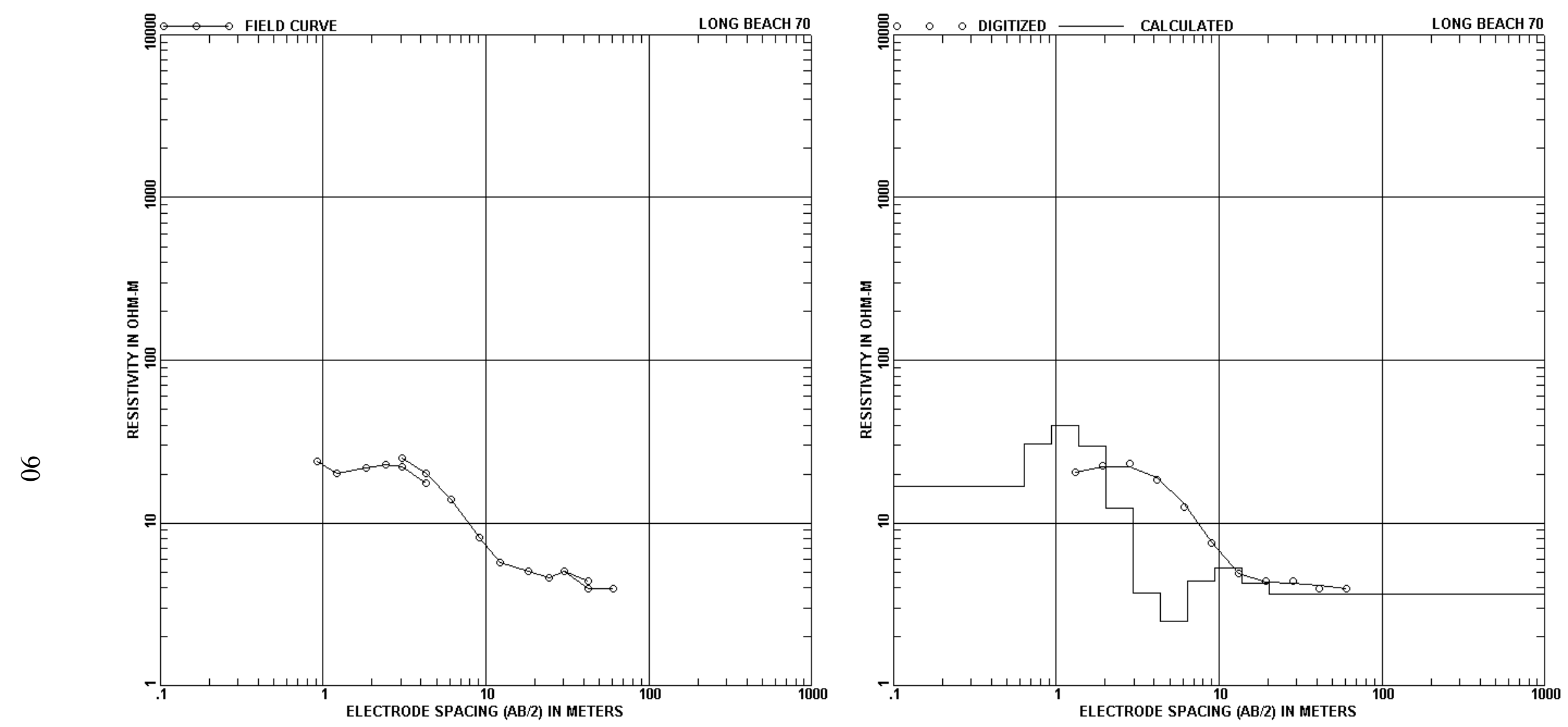

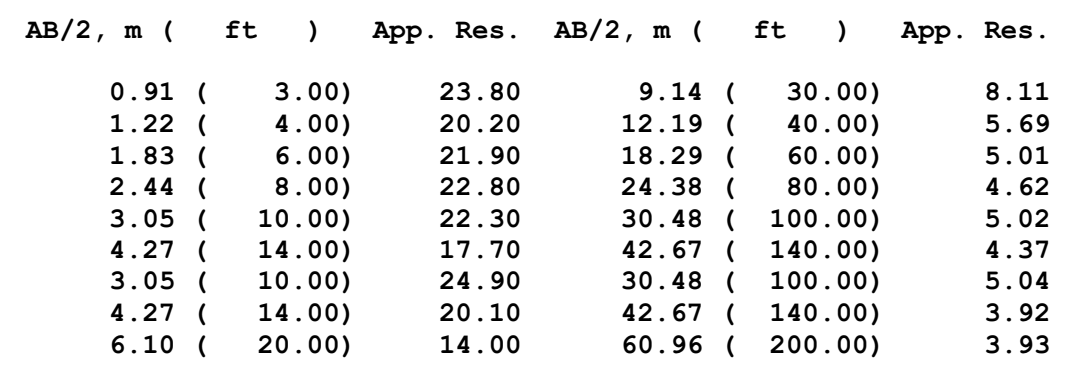

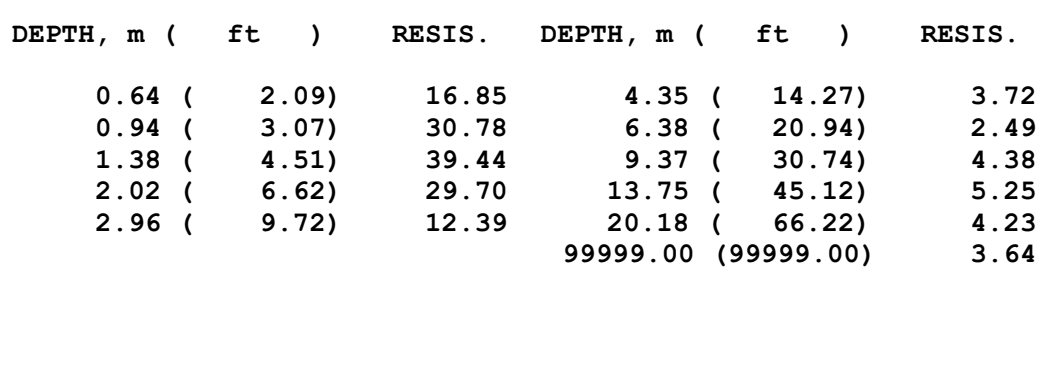



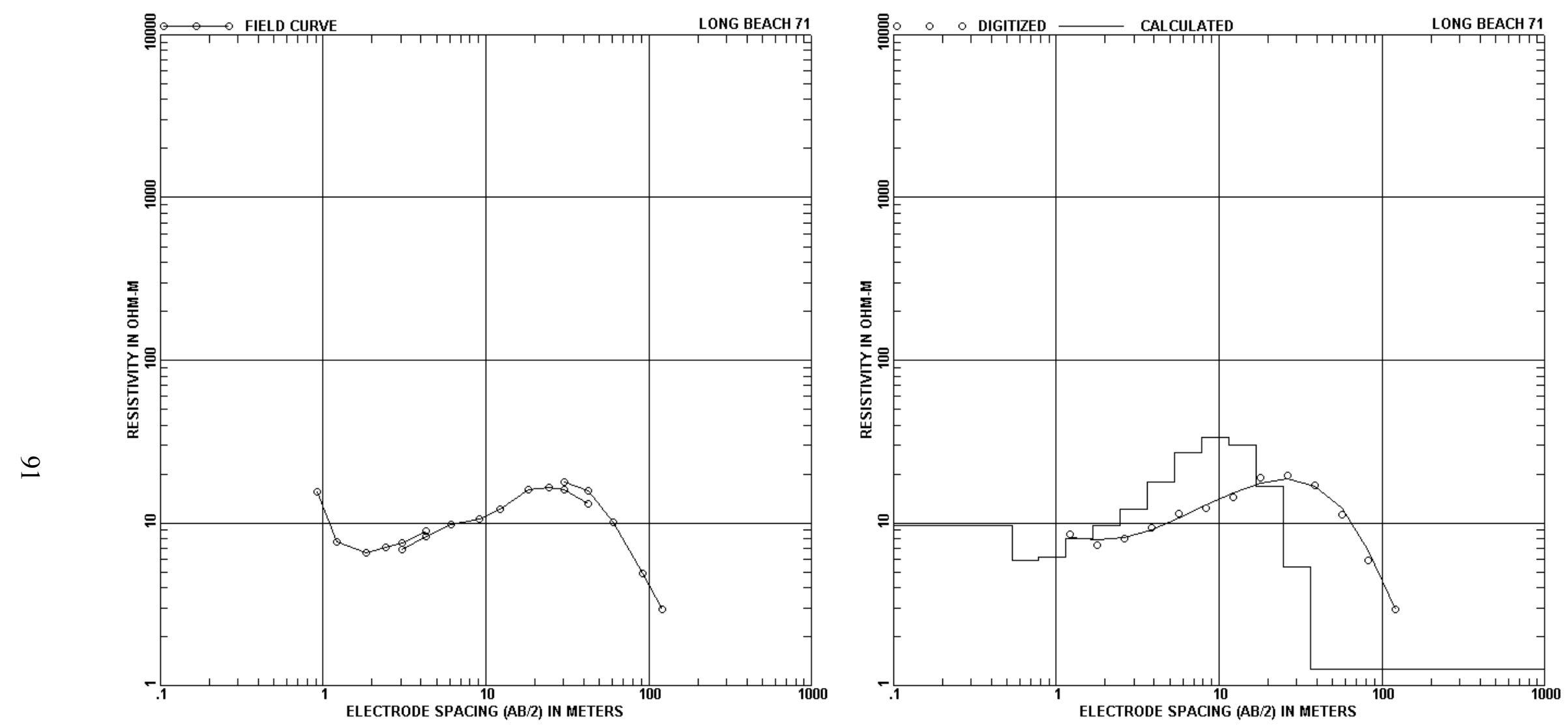

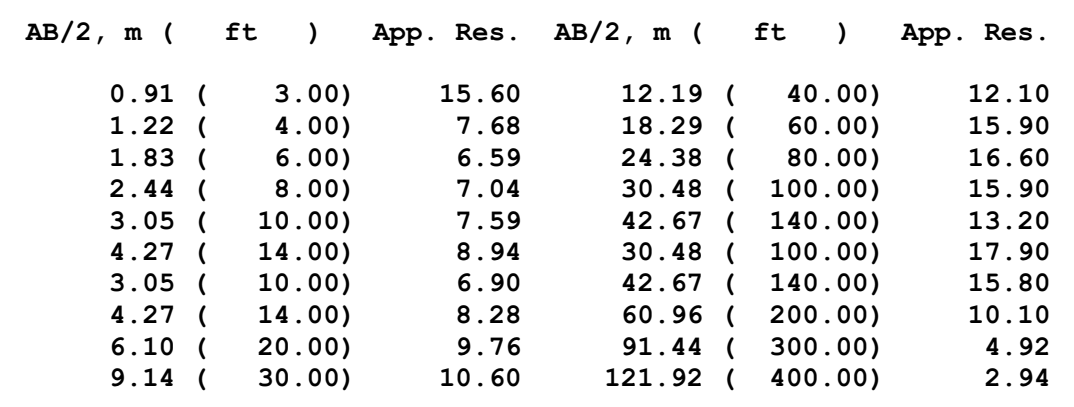

\begin{tabular}{|c|c|c|c|c|c|}
\hline DEPTH， m ( & ft, & RESIS. & DEPTH， m & $1 \mathrm{ft}$ & RESIS. \\
\hline 0.53 & 1.75) & 9.70 & 5.33 & $17.50)$ & 17.88 \\
\hline 0.78 & $2.57)$ & 5.86 & 7.83 & $25.68)$ & 26.98 \\
\hline 1.15 & $3.77)$ & 6.13 & 11.49 & $37.69)$ & 33.69 \\
\hline 1.69 & $5.53)$ & 7.97 & 16.86 & $55.33)$ & 30.12 \\
\hline 2.48 & $8.12)$ & 9.57 & 24.75 & $81.21)$ & 16.88 \\
\hline 3.63 & 11.92) & 12.06 & 36.33 & $(119.20)$ & 5.33 \\
\hline & & & 99999.00 & $(99999.00)$ & 1.27 \\
\hline
\end{tabular}



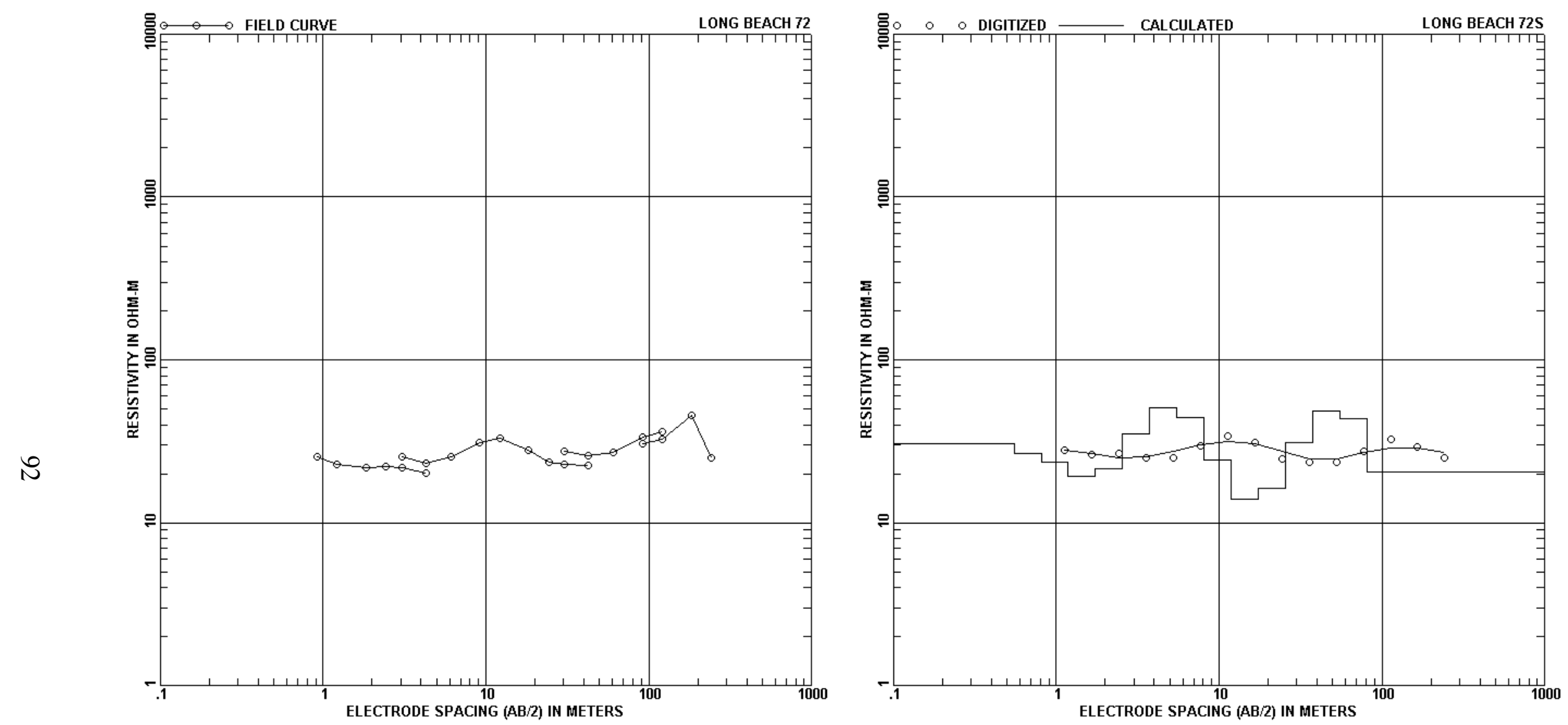

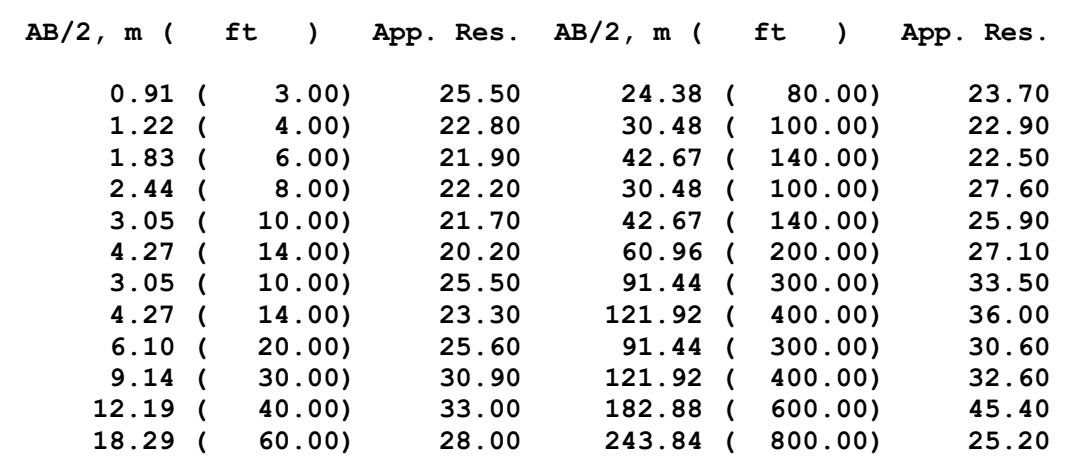

DEPTH, $m$ ( ft ) RESIS, DEPTH $m($ ft

$\begin{array}{rlr}0.55 & ( & 1.80) \\ 0.81 & ( & 2.65) \\ 1.19 & ( & 3.89) \\ 1.74 & ( & 5.71) \\ 2.55 & ( & 8.38) \\ 3.75 & ( & 12.29) \\ 5.50 & ( & 18.05)\end{array}$

30.40
26.74
23.47
19.14
21.39
35.15
50.76

$8.07(26.49)$
$11.85(38.88)$

$11.85(38.88)$

$17.39(57.07)$

$25.53(83.76)$

$37.47(122.95)$

$55.01(180.47)$

$80.74(264.89)$

$99999.00(99999.00)$
RESIS.

44.55 24.41 14.01

16.29

31.08

31.08

48.65 43.30
20.36 

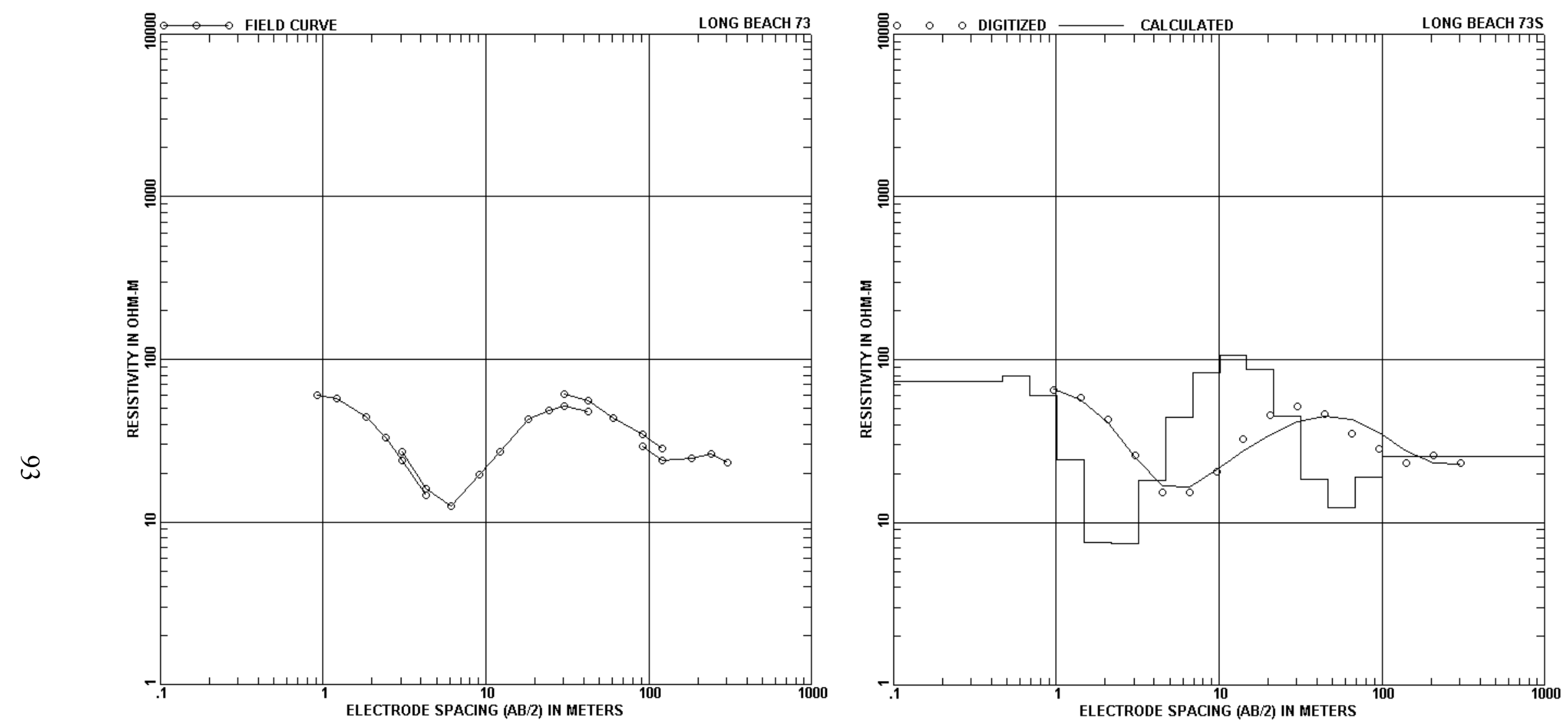

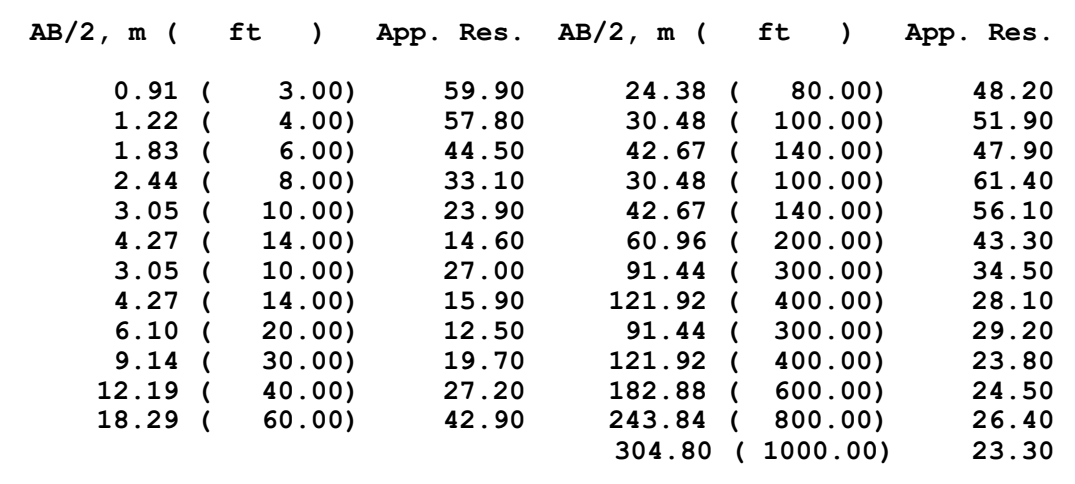

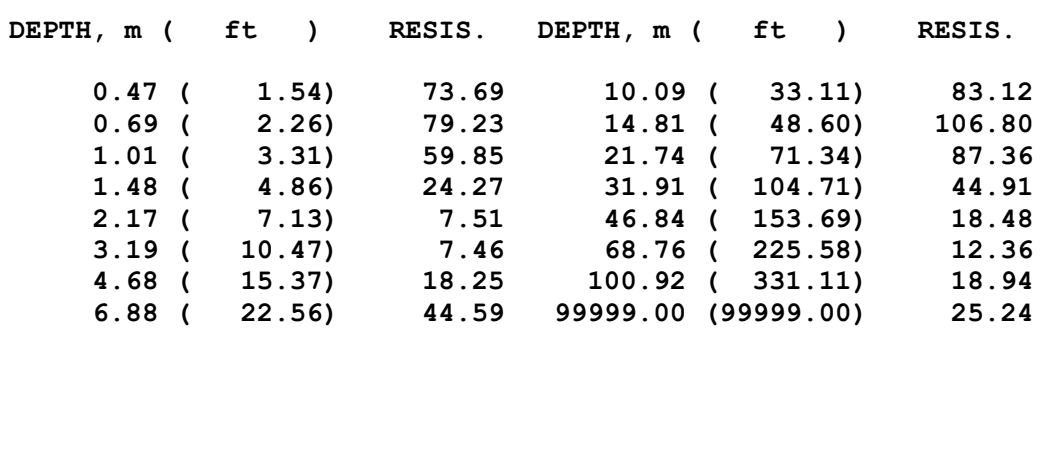



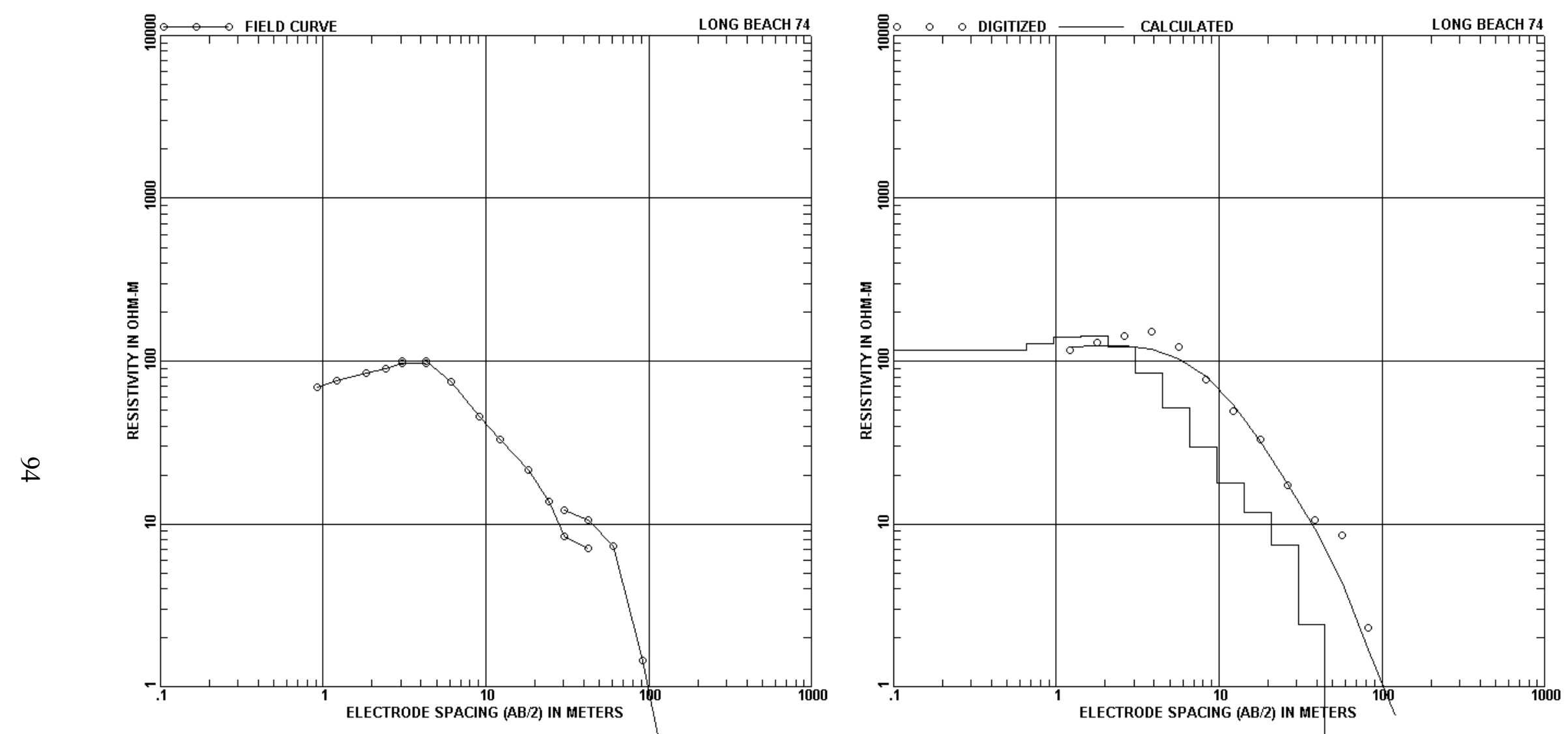

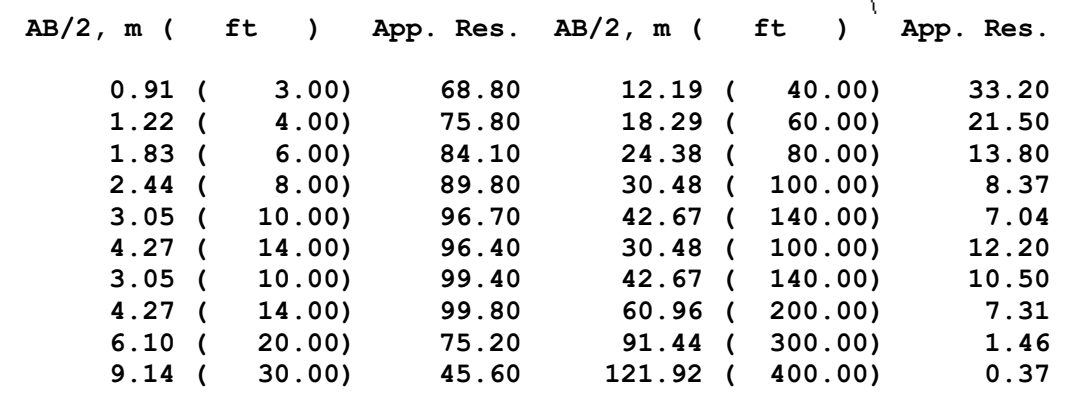

\begin{tabular}{|c|c|c|c|c|c|}
\hline DEPTH， m ( & ft $)$ & RESIS. & DEPTH， m ( & ft & RESIS. \\
\hline 0.661 & $2.16)$ & 117.13 & 6.58 & $21.60)$ & 51.32 \\
\hline 0.971 & $3.17)$ & 128.59 & 9.66 & $31.70)$ & 29.71 \\
\hline $1.42 i$ & $4.65)$ & 141.08 & 14.18 & $46.54)$ & 17.73 \\
\hline $2.08 i$ & $6.83)$ & 142.46 & 20.82 & $68.31)$ & 11.82 \\
\hline $3.06 i$ & $10.03)$ & 121.39 & 30.56 & $100.26)$ & 7.46 \\
\hline 4.491 & $14.72)$ & 84.75 & 44.85 & $(147.16)$ & 2.43 \\
\hline & & & 99999.00 & $(99999.00)$ & 0.38 \\
\hline
\end{tabular}



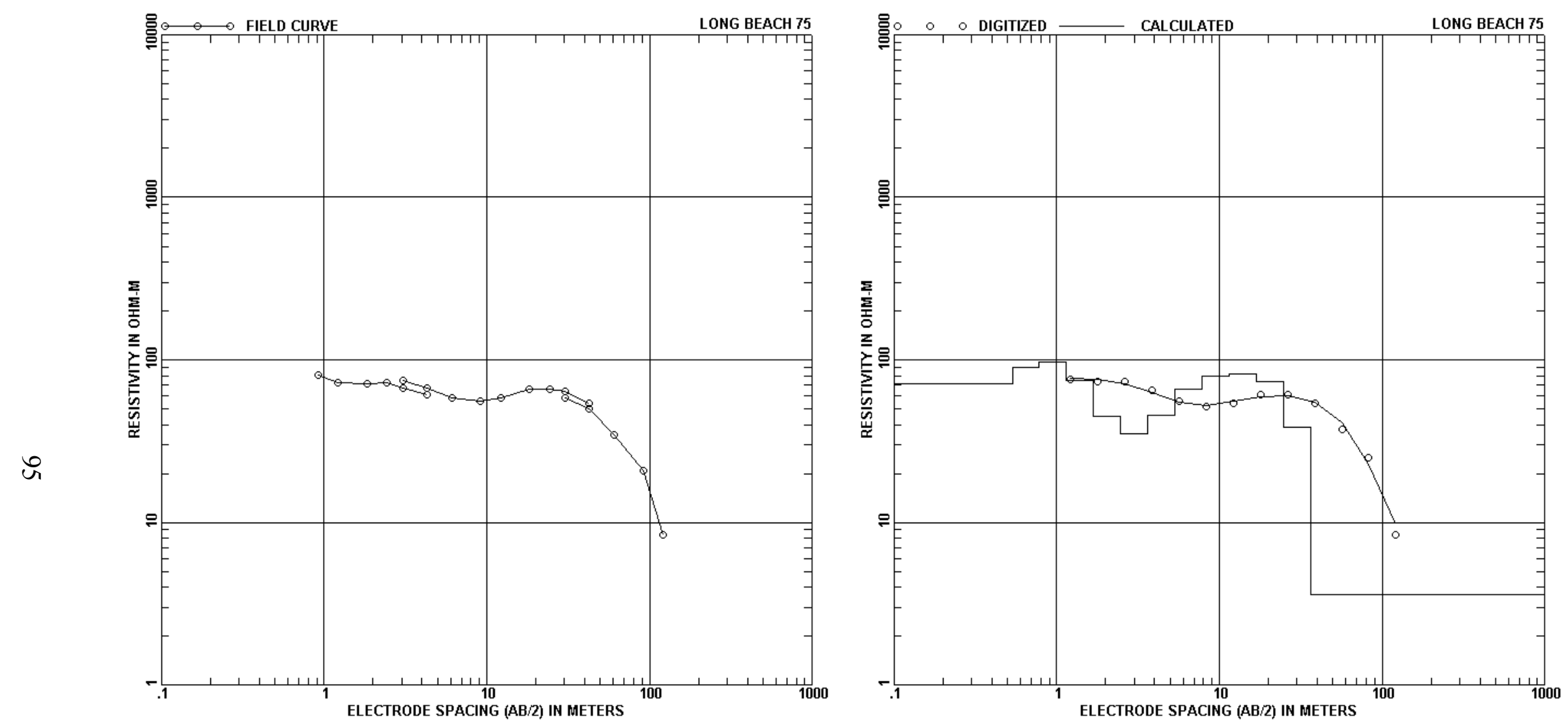

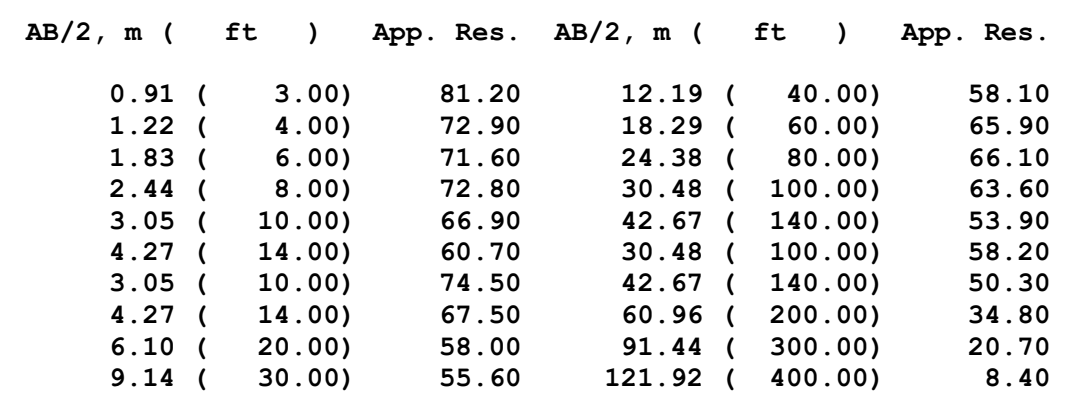

\begin{tabular}{|c|c|c|c|c|c|}
\hline DEPTH， m ( & ft, & RESIS. & DEPTH， m & $1 \mathrm{ft}$ & RESIS. \\
\hline 0.53 & 1.75) & 71.59 & 5.33 & $17.50)$ & 45.81 \\
\hline 0.78 & $2.57)$ & 89.95 & 7.83 & $25.68)$ & 65.65 \\
\hline 1.15 & $3.77)$ & 97.55 & 11.49 & $37.69)$ & 79.60 \\
\hline 1.69 & $5.53)$ & 75.05 & 16.86 & $55.33)$ & 82.16 \\
\hline 2.48 & $8.12)$ & 44.67 & 24.75 & $81.21)$ & 73.13 \\
\hline 3.63 & 11.92) & 35.11 & 36.33 & $(119.20)$ & 38.44 \\
\hline & & & 99999.00 & $(99999.00)$ & 3.58 \\
\hline
\end{tabular}



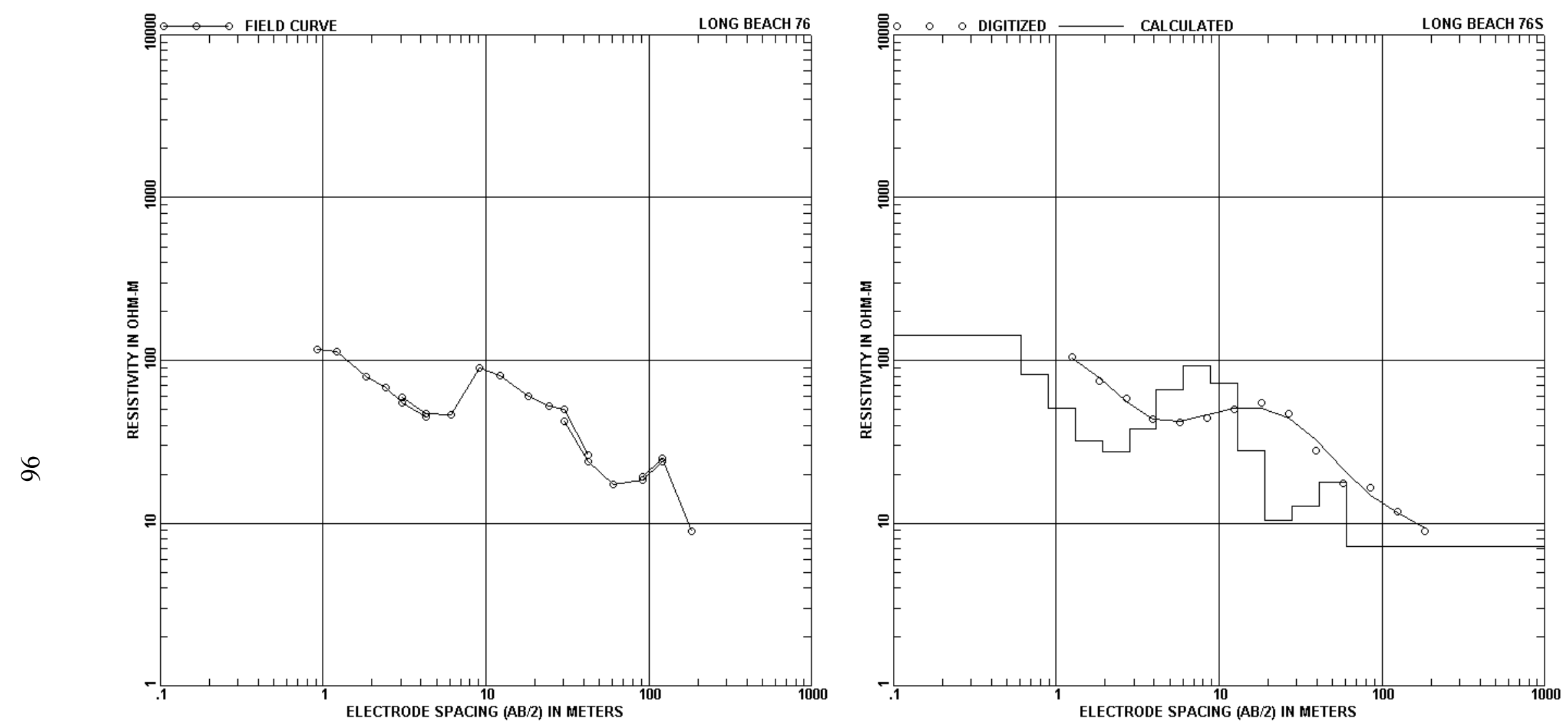

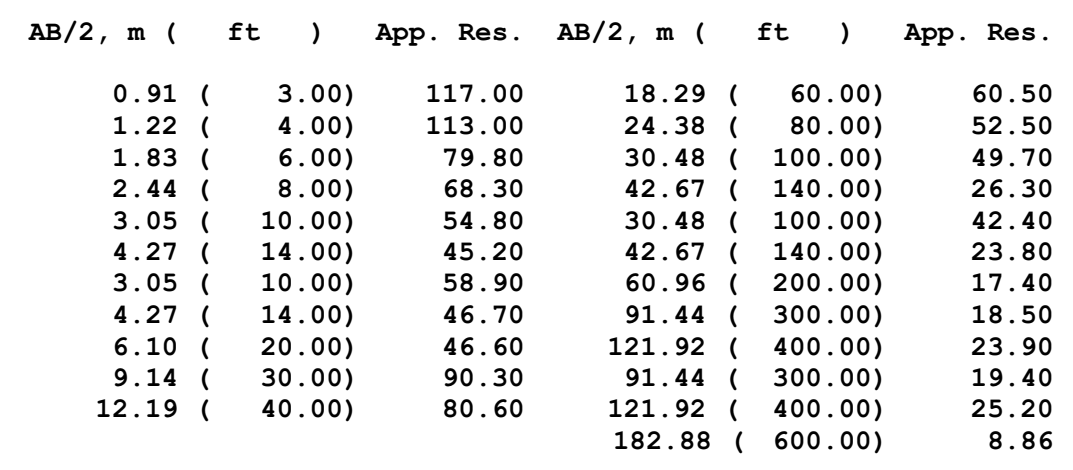

DEPTH， m ( ft ) RESIS, DEPTH $m$ ( ft

$\begin{array}{rlr}0.61 & ( & 1.99) \\ 0.89 & ( & 2.92) \\ 1.30 & ( & 4.28) \\ 1.91 & ( & 6.28) \\ 2.81 & ( & 9.22) \\ 4.13 & ( & 13.53) \\ 6.06 & ( & 19.87)\end{array}$

142.58

82.49

50.76

32.13

27.29

38.04

66.04
$8.89(29.16)$

$13.05(42.80)$

$19.15(62.82)$

28.11 ( 92.21)

41.25 ( 135.35)

$60.55(198.66)$

$99999.00(99999.00)$
RESIS.

92.35

72.23

28.09

10.48

12.64

17.80
7.15

8.50

19.40

8.86 

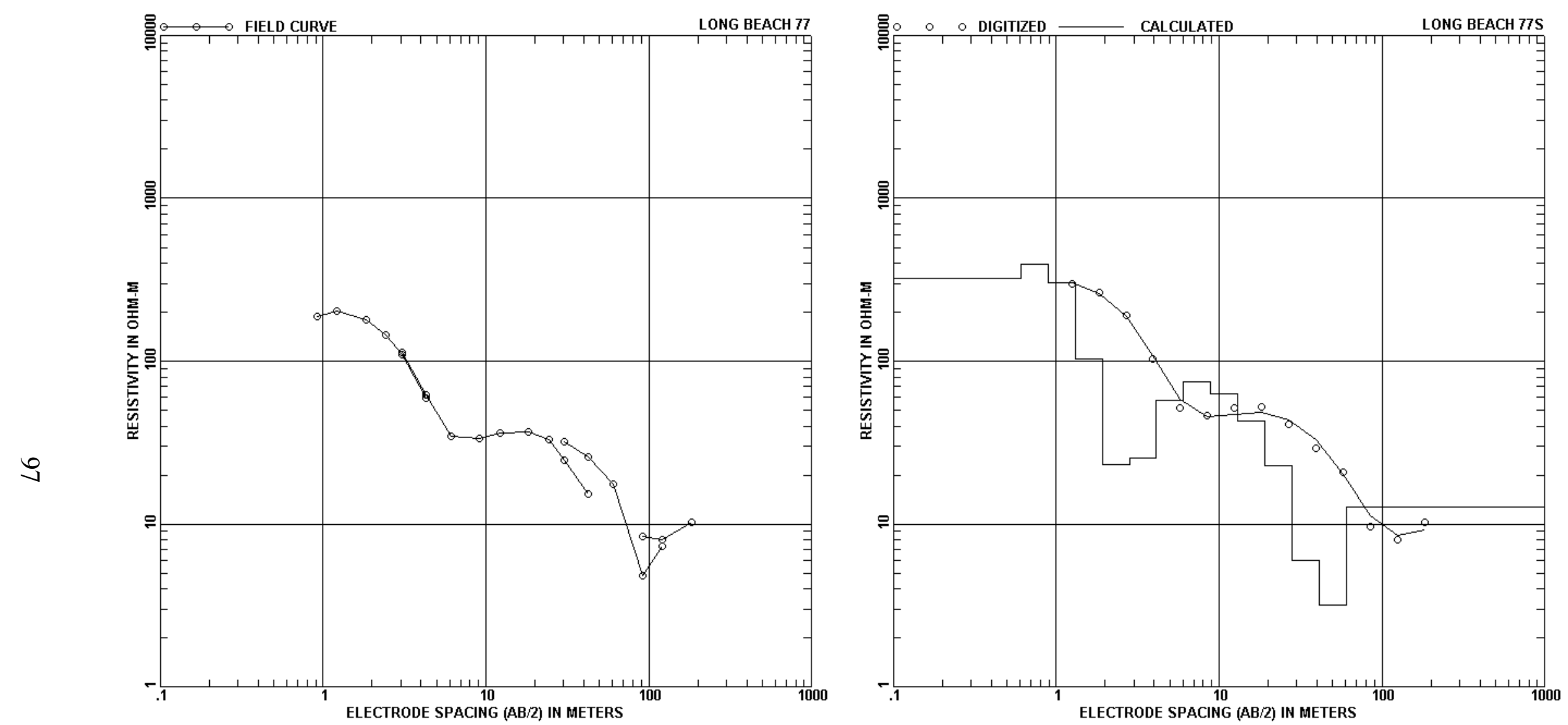

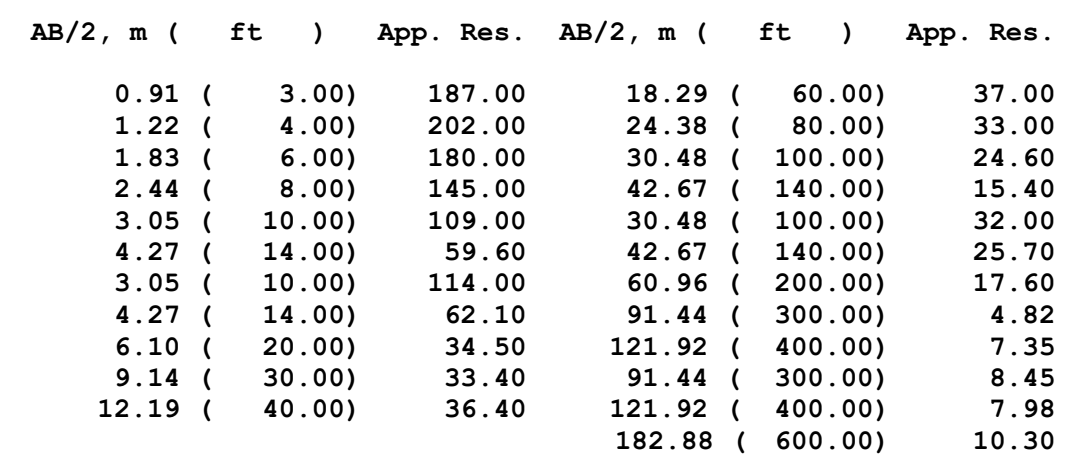

DEPTH, $m$ ( ft ) RESIS, DEPTH, $m$ ( ft

\begin{tabular}{|c|c|c|c|c|c|}
\hline 0.61 & 1.99) & 323.22 & 8.89 & $29.16)$ & 75.24 \\
\hline 0.89 & $2.92)$ & 391.80 & 13.05 & $42.80)$ & 62.66 \\
\hline 1.30 & $4.28)$ & 305.13 & 19.15 & $62.82)$ & 43.20 \\
\hline 1.91 & $6.28)$ & 102.92 & 28.11 & $92.21)$ & 22.87 \\
\hline 2.81 & $9.22)$ & 23.01 & 41.25 & $135.35)$ & 6.01 \\
\hline 4.13 & $13.53)$ & 25.43 & 60.55 & $198.66)$ & 3.18 \\
\hline 6.06 & 19.87) & 57.59 & 99999.00 & $(99999.00)$ & 12.71 \\
\hline
\end{tabular}



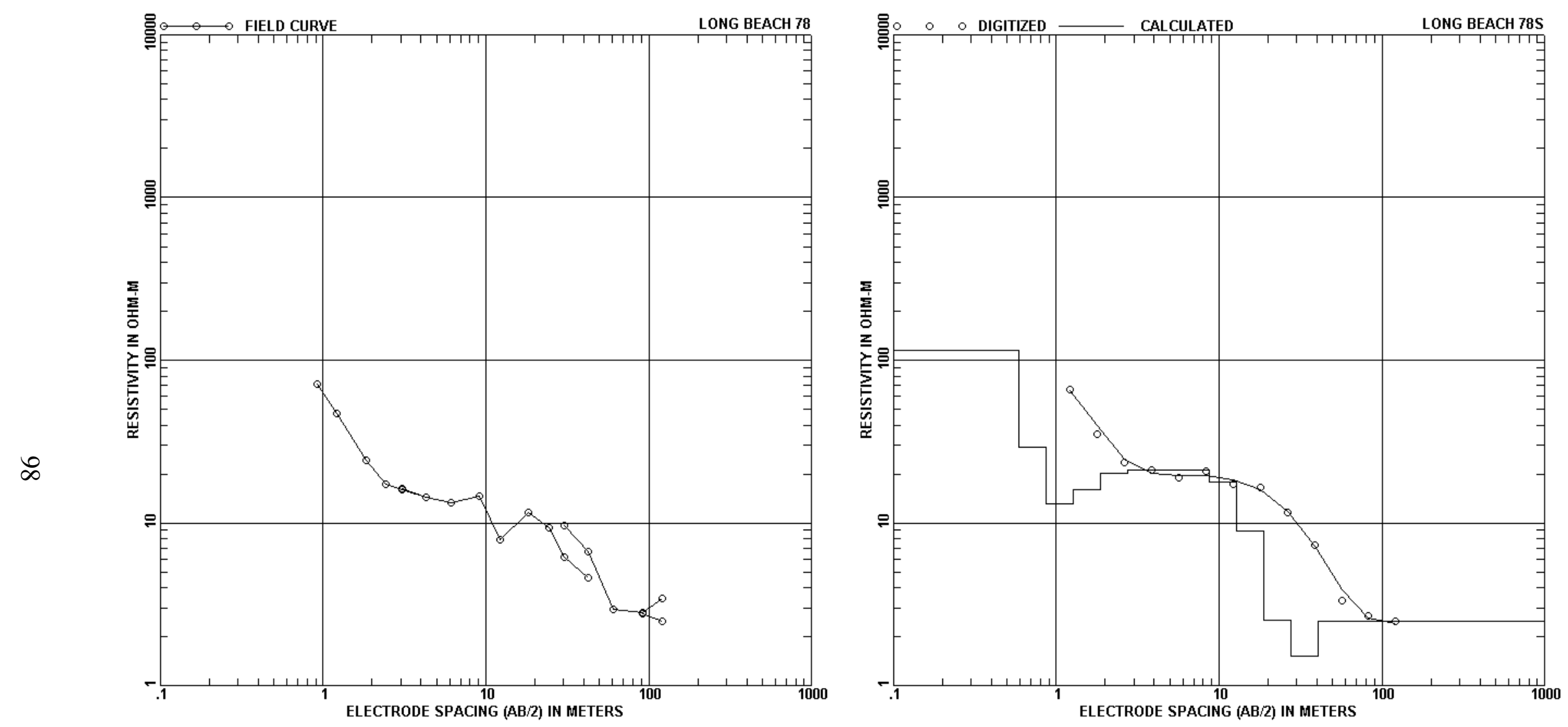

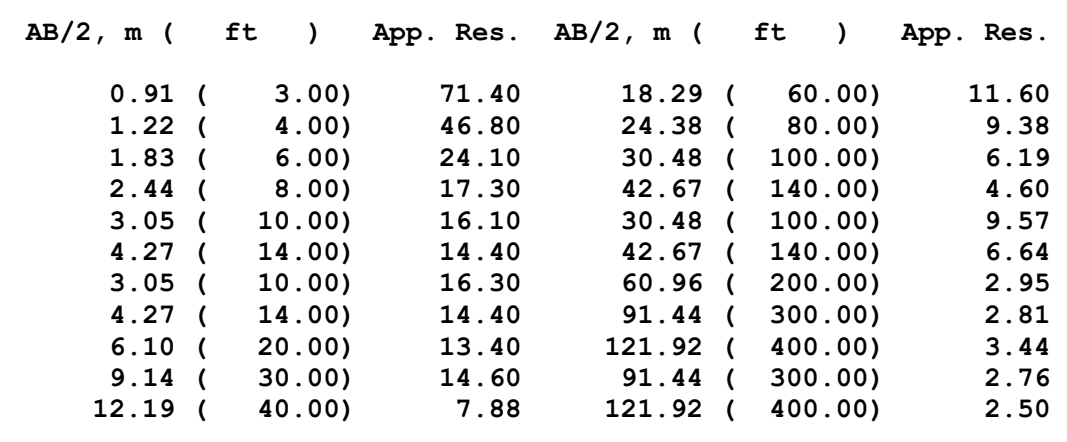

\begin{tabular}{|c|c|c|c|c|c|}
\hline DEPTH， m ( & ft & RESIS. & DEPTH， m ( & ft & RESIS. \\
\hline 0.591 & $1.94)$ & 115.14 & 5.93 & $19.44)$ & 21.02 \\
\hline $0.87 i$ & $2.85)$ & 29.08 & 8.70 & $28.53)$ & 21.09 \\
\hline 1.28 ( & $4.19)$ & 13.13 & 12.77 & $41.88)$ & 17.96 \\
\hline $1.87 \%$ & $6.15)$ & 16.10 & 18.74 & $61.47)$ & 8.94 \\
\hline $2.75 i$ & $9.02)$ & 20.25 & 27.50 & $90.23)$ & 2.52 \\
\hline 4.04 & $13.24)$ & 21.29 & 40.37 & $132.44)$ & 1.51 \\
\hline & & & 99999.00 & $(99999.00)$ & 2.47 \\
\hline
\end{tabular}

